\title{
MACHINE-AIDED ANALYSIS OF VOTE PRIVACY USING COMPUTATIONALLY COMPLETE SYMBOLIC ATTACKER
}

\author{
A Dissertation \\ presented to \\ the Faculty of the Graduate School \\ at the University of Missouri-Columbia \\ In Partial Fulfillment \\ of the Requirements for the Degree \\ Doctor of Philosophy \\ by \\ AJAY KUMAR EERALLA \\ Dr. Rohit Chadha, Dissertation Supervisor
}

DEC 2019 
(c) Copyright by Ajay Kumar Eeralla 2019

All Rights Reserved 
The undersigned, appointed by the Dean of the Graduate School, have examined the dissertation entitled:

MACHINE-AIDED ANALYSIS OF VOTE PRIVACY USING COMPUTATIONALLY COMPLETE SYMBOLIC ATTACKER

presented by Ajay Kumar Eeralla, a candidate for the degree of Doctor of Philosophy, and hereby certify that, in their opinion, it is worthy of acceptance.

Dr. Rohit Chadha

Dr. William L. Harrison

Dr. Gergei Bana

Dr. Prasad Calyam 


\section{Dedication}

To my parents for their love and faith in me. 


\section{Acknowledgements}

This thesis would not have completed without the help of my supervisor, and I would like to express my sincere gratitude to Dr. Rohit Chadha for his patience, support, enthusiasm, and encouragement. He has introduced me and taught courses such as Formal Engineering Methods for Software and Security and an Advanced Course in Cryptography, which helped me in improving my knowledge in this area of research. Dr. Chadha's guidance helped me not only in doing my research and the writing of this dissertation but also in improving my skillset in general. I'm fortunate to have Professor Rohit Chadha as my faculty advisor.

I would also like to thank Professor Gergei Bana for collaborating with us and for being one of the members of my dissertation committee. As one of the inventors of the computationally complete symbolic attacker model, Dr. Bana helped us understanding the technique and completing the proofs of vote privacy in that model.

Furthermore, I would like to thank my other thesis committee members Professor William Harrison and Professor Prasad Calyam for their time and comments that helped me in improving my dissertation.

My sincere thanks also go to the faculty members of the Department of Computer Science for supporting me with the teaching assistantship(s) during my PhD program here at the University of Missouri (Mizzou).

This work is partially supported by the National Science Foundation (NSF) under

the grants CNS 1314338 and CNS 1553548. I would like to thank NSF for supporting me during my Ph.D. studies here in the United States. Without their support, it would not have possible attending the conferences and workshops. 
Last but not least, I also like to thank my amazing parents and my brother for having faith in me and for their enormous support not only in my studies but also in other aspects of my life. Of course, my wife is a great supporter of mine in pursuing my goals.

I have had a great time hanging out with the people from the Center for High Assurance Computing (CHACo) lab in the Mizzou, namely, Chris, Qian Li, Tom, Seth, Adam, Rohit, and Bill, and I would like to thank them for making me feel comfortable in the lab.

Columbia, Missouri

September 25, 2019 


\section{Table of Contents}

$\begin{array}{ll}\text { Acknowledgements } & \text { ii }\end{array}$

List of Tables viii

List of Figures $\quad$ ix

ABSTRACT $\quad$ xi

CHAPTER

1 Introduction $\quad 1$

1.1 The Context . . . . . . . . . . . . . . . . . 1

1.2 Our Contributions . . . . . . . . . . . . . . . 4

1.2.1 Analyzing FOO voting protocol using CCSA . . . . . . . . . 4

1.2.2 Mechanizing the proofs using Coq . . . . . . . . . . . . . 6

1.3 Related Works . . . . . . . . . . . . . . . . . 7

1.4 Outline of the Dissertation . . . . . . . . . . . . . . . . . 8

2 Background $\quad 9$

2.1 The Symbolic Model . . . . . . . . . . . . . . . . . . . . . . . . . . . 11

2.2 The Computational Model . . . . . . . . . . . . . . . . . . . . . 12

2.3 Computational Soundness . . . . . . . . . . . . . . 15

2.4 Computationally Complete Symbolic Attacker . . . . . . . . . . . 16 
$2.4 .1 \quad \operatorname{Syntax} \ldots \ldots \ldots \ldots \ldots \ldots \ldots \ldots$

$2.4 .2 \quad$ Semantics . . . . . . . . . . . . . . . . . . . 19

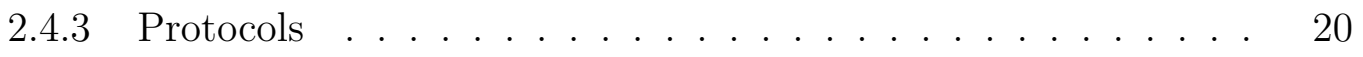

2.4.3.1 The transition system . . . . . . . . . . . 20

2.4.3.2 Executions and indistinguishability . . . . . . . 24

2.4.3.3 The soundness theorem _ . . . . . . . . . 26

2.4.4 Computationally Valid Axioms _. . . . . . . . . 27

3 FOO Protocol and its Analysis using CCSA 30

3.1 Introduction . . . . . . . . . . . . . . . . . . 31

$3.2 \quad$ FOO Voting Protocol $\ldots \ldots \ldots \ldots \ldots \ldots$

3.2 .1 Anonymous Channel . . . . . . . . . . . . . . . . . . . 36

3.3 Computational Modeling of the FOO protocol . . . . . . . . . . 37

3.3 .1 Commitments . . . . . . . . . . . . . . . . . . 38

3.3 .2 Blind Digital Signatures _ . . . . . . . . . . . . . . . . 39

3.3.3 Digital Signatures . . . . . . . . . . . . . . . . . . . . 42

3.3 .4 Encryptions . . . . . . . . . . . . . . . . . . . 44

3.4 Vote privacy for the FOO protocol $\ldots \ldots \ldots \ldots$

3.4.1 Formalization of Vote Privacy . . . . . . . . . . . 46

3.4 .2 Attacks on the FOO Protocol . . . . . . . . . . . . 49

3.4.2.1 A computational attack due to unequal vote lengths 50

3.4.2.2 A computational attack due to unequal blind signature lengths . . . . . . . . . . . . . . . 51

3.4.2.3 A Dolev-Yao style replay attack . . . . . . . . . . . 51

3.4.2.4 A man-in-the-middle attack . . . . . . . . . . . 52

3.5 Vote Privacy for the FOO Protocol in CCSA Framework . . . . . . 53

3.5.1 Cryptographic Primitives and their Modeling . . . . . . . 53

3.5 .2 Proofs of Validity . . . . . . . . . . . . . . . . . . . . 59 
3.5.3 Other Function Symbols and Assumptions . . . . . . . . . . 63

3.5.4 Modeling the Vote Privacy Security Game . . . . . . . . . . 65

3.6 Summary . . . . . . . . . . . . . . . . . 69

4 Proofs of Vote Privacy $\quad 70$

4.1 Auxiliary Theorems . . . . . . . . . . . . . . . . 70

4.1.1 Notations and Conventions . . . . . . . . . . . . . 78

4.1.2 Extending Blindness axiom . . . . . . . . . . . . 81

4.2 Proofs of Vote Privacy . . . . . . . . . . . . . 86

4.2.1 Authentication Phase . . . . . . . . . . . . 87

4.2.2 Voting and Opening Phases . . . . . . . . . . . . 87

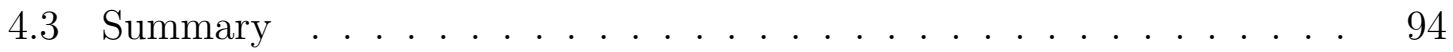

5 Mechanization $\quad 95$

5.1 Formalizing CCSA Framework in Coq . . . . . . . . . . . 96

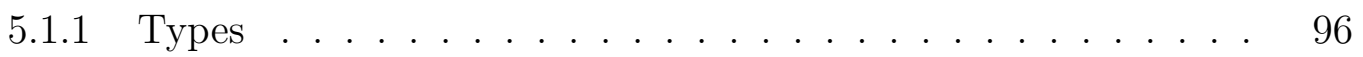

5.1 .2 Strengthening Induction . . . . . . . . . . . . . . 98

5.1 .3 Indistinguishability Relation . . . . . . . . . . . . . . . 103

5.1.4 Formalization of Equality . . . . . . . . . . . . . 105

5.1.5 Formalization of the Core Axioms . . . . . . . . . . . . . 107

5.2 Formalizing the Proofs of the Vote Privacy . . . . . . . . . . . . . 110

5.3 Authenticated Diffie-Hellman Key Exchange . . . . . . . . . . . . . . 117

5.3.1 Modeling DDH assumption in the CCSA framework . . . . . . 119

5.3.2 Modeling UF-CMA in the CCSA framework . . . . . . . . . . 120

5.3.3 Formalizing the authentication of the STS protocol . . . . . . 120

5.3.4 Formalizing secrecy of the STS protocol . . . . . . . . . . 127

5.4 Summary . . . . . . . . . . . . . . . . . . . 128

6 Conclusion $\quad 129$ 
$\begin{array}{ll}\text { Bibliography } & 130\end{array}$

VITA

139 


\section{List of Tables}

Table $\quad$ Page

2.1 Core Axioms . . . . . . . . . . . . . . . . 27

3.1 Axioms that formalize the additional assumptions of the primitives . 64 


\section{List of Figures}

Figure

Page

2.1 Needham-Schroeder protocol . . . . . . . . . . . . . . . . 9

2.2 Man-in-the-middle attack on NS protocol . . . . . . . . . . . . . . 10

2.3 Needham-Schroeder-Lowe protocol . . . . . . . . . . . . . . . . . 11

2.4 Diffie-Hellman key exchange protocol . . . . . . . . . . . . . . . . 22

2.5 DH key exchange protocol with an initiator session and a responder

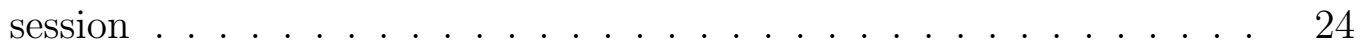

3.1 FOO Voting Protocol . . . . . . . . . . . . . . . . . 35

3.2 The hiding experiment $\operatorname{Hiding}_{\text {com }}^{\mathcal{A}}(n)$ of the commitments . . . . . 39

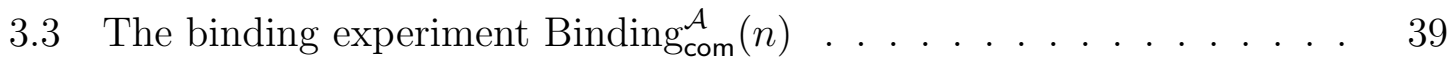

3.4 The blind signing experiment $\operatorname{Blind}_{S^{*}}^{\mathrm{B}}(n) \ldots \ldots \ldots . \ldots . \ldots . \ldots 4$

3.5 The signature experiment Signforge $\mathrm{e}_{\mathcal{A}, \Pi}^{\mathrm{cma}}(n) \ldots \ldots . \ldots . \ldots . . \ldots 43$

3.6 The adaptive CCA experiment $\operatorname{PubK}_{\mathcal{A}, \Pi}^{\mathrm{cca} 2}(n) \ldots \ldots . . . . . . . \quad 45$

3.7 Axioms that formalize the computational assumptions of the commit-

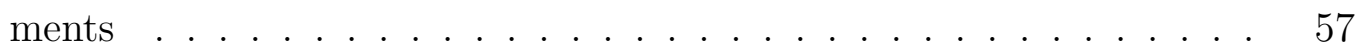

3.8 Axiom that formalizes the blindness property of the Blind Signatures 58

3.9 Shuffle axiom . . . . . . . . . . . . . . . . . . . . . 58

3.10 Modeling IND-CCA2 of encryptions in the CCSA framework . . . . 59

3.11 Authentication phase of the security game $\Pi_{b} \ldots \ldots$. . . . . . 66 
3.12 Voting Phase of the FOO protocol . . . . . . . . . . . 67

3.13 Opening Phase of the FOO protocol . . . . . . . . . . . 68

5.1 Authenticated Diffie-Hellman key exchange protocol . . . . . . . . . 118

5.2 Axiom that formalizes the DDH assumption . . . . . . . . . . 119

5.3 Axiom that formalizes the UF-CMA assumption of signatures $\ldots \ldots$

5.4 Authenticated DH key exchange protocol with two agents A and B . 123 


\section{ABSTRACT}

Security protocols employ cryptographic primitives such as encryption and digital signatures to provide security guarantees of confidentiality and authenticity in the presence of malicious attackers. Due to the complexities of cryptographic primitives, subtle nature of the security guarantees and asymmetry of communication over the internet, their design tends to be error-prone. Thus, formal methods are often used to establish whether the protocols actually achieve their guarantees. The analysis can be either carried out in the Dolev-Yao model, where the cryptographic primitives are assumed to be perfect, and the attacker tries to exploit the logical errors to compromise the security of the protocols or in the provable security model, where the attacker can, in addition, break the cryptographic primitives with negligible probability. The provable security model provides better guarantees.

We consider formalizing and verifying the security property of vote privacy for electronic voting protocols in the provable security model. As an example, we consider analyzing the FOO electronic voting protocol introduced by Fujioka, Okamoto, and Ohta. Several automated analyses have been carried for the FOO protocol in the Dolev-Yao model, and the protocol is secure in the Dolev-Yao model. The protocol uses commitments, blind signatures, and anonymous channels to achieve vote privacy. The Dolev-Yao analyses also assume the existence of perfectly anonymous channels.

We carried out the analysis of the security protocol using the Computationally Complete Symbolic Attacker (CCSA) technique, which allows the establishment of 
proofs of security guarantees using deduction in first-order logic. Unlike the DolevYao analyses of the protocol, we assume neither perfect cryptography nor existence of perfectly anonymous channels. We model the anonymous communication using a mix-net server who is responsible for checking if the received messages are distinct and outputting the decrypted messages in a lexicographic order. Our analysis reveals new attacks on vote privacy including an attack that arises due to the inadequacy of the blindness property of blind signatures and a Dolev-Yao style attack that arises due to the modeling of the anonymous communication as a mix-net server. With additional assumptions and modifications of the protocol, we were able to show that the protocol satisfies vote privacy in the sense that switching votes of two honest voters is undetectable to the attacker.

In order to achieve higher assurances, we mechanized the CCSA technique in Coq, an interactive theorem-prover $\left[\mathrm{BC} 04, \mathrm{PdAC}^{+} 17\right]$ developed using the specification language Gallina. We demonstrate the effectiveness of our mechanization with the verification of authentication and secrecy guarantees of the Authenticated DiffieHellman key exchange protocol. Finally, we prove the key lemmas of the proof of voter privacy for the FOO protocol in Coq. 


\section{Chapter 1}

\section{Introduction}

\section{$1.1 \quad$ The Context}

With the evolution of the Internet and the increasing number of online users, the necessity of deployment of security protocols is increasingly ubiquitous on the World Wide Web. The security protocols employ cryptographic primitives such as encryption and digital signatures to provide security guarantees of confidentiality and authenticity in the presence of malicious attackers. A well-known example is the Transport Layer Security (TLS) protocol which is a foundational part of the protocols including HTTPS, SSH, IPsec, and SMTPS. The TLS protocol is used to secure the communication between a client and a server over the Internet.

Unfortunately, security protocols often have flaws in their design due to the complexities of cryptographic primitives and subtle nature of the security guarantees and asymmetry of communication over the internet. For example, the TLS protocol has been successfully attacked several times in the last decade $\left[\mathrm{BDF}^{+} 14, \mathrm{BBD}^{+} 17\right.$, $\left.\mathrm{ABD}^{+} 15\right]$.

Noting the fact that security protocols are error-prone, it is essential to analyze them with respect to attacker models that formalize the capabilities of the at- 
tacker. Primarily there are two well-known attacker models from the literature, the one due to Dolev and Yao [DY83] and the other due to Goldwasser, Micali, Rivest, and Yao [Gol84, GMR88, Yao82]. The former is called the Dolev-Yao or symbolic model, whereas the latter is known as the computational or provable security model. The Dolev-Yao model assumes perfect black-box cryptography and tries to find logical errors using the theory of logic and programming languages. The attacker capabilities are often expressed through deduction rules explicitly and allowed to perform any computation using only these rules. On the other hand, the computational model leverages the theory of computational complexity, and the attacker is modeled as a polynomially-bounded probabilistic Turing machine without restricting to a fixed set of operations.

The Dolev-Yao model is simpler and amenable to verify security properties automatically. Thus, there are several automated tools available for proving security, such as ProVerif [Bla05], Scyther [Cre08], and TAmarin [SMCB12]. On the other hand, although the computational model offers stronger security guarantees than the Dolev-Yao, the proofs of security in this model are complicated and erring. There are only few tools (semi-automatic) available such as CRYPTOVERIF [Bla08a] and EASYCRYPt [BGHZB11]. However, these tools have several limitations. Name to few; the provers often fail to complete proofs in the computational model even in the case of secure protocols. When the provers do not complete the proof, It is not clear to the user if it is because of a protocol flaw, or it is due to limitations of the prover.

A third approach developed by Abadi and Rogaway (see [AR02, BPW03], for example), which aimed at bridging the gap between the Dolev-Yao and the computational model. It is known as the computational soundness approach, essentially says, under some conditions, the security of a protocol in the Dolev-Yao implies the security in the provable security model. However, this approach has several limita- 
tions. First, these results often require stronger assumptions about computational implementations, which makes one question their utility. Second, the proofs in this approach are not modular, adding new primitives requires to prove the whole system again. As the proofs in this approach tend to be involved, this imposes a significant burden. Due to these problems, the research in this direction is not much progressive if not abandoned by now.

In order to overcome the discrepancy as mentioned above, Bana and Comon proposed a new approach(see [BCL12]) that aimed at establishing computational guarantees while leveraging the symbolic techniques with reasonable assumptions about the cryptographic primitives. The authors named it as Computationally Complete Symbolic Attacker (CCSA). Although the framework has only been used for verifying reachability properties initially [BAS12a, BHO13], with [BCL14, Sce15], the creators demonstrated the practicality of the framework in the verification of equivalence properties.

The CCSA framework is similar to the Dolev-Yao framework in the sense that explicit symbolic adversarial messages are created. The Dolev-Yao framework specifies all rules that the attacker can use to create new messages from what he has seen, and the protocol agents use pattern matching to check whether messages coming from the attacker have the correct form. On the other hand, in the CCSA framework, each message from the attacker is modeled by a function $f_{i}$ applied to the sequence of messages that the attacker has seen thus far. Pattern matching is replaced by applying function symbols on the term coming from the attacker. Limitations on attacker capabilities originating from computational assumptions on the primitives are specified as a set of axioms $A$ in first-order logic based on a single indistinguishability predicate which represents computational indistinguishability of sequences of messages. Computationally, a security property of a protocol $\Pi$ is formulated as the computational indistinguishability of two protocols $\Pi_{1}$ and $\Pi_{2}$ constructed (depending on the 
security property) from the original $\Pi$.

In the CCSA framework, the security translates to the validity of the formula obtained by applying the indistinguishability predicate on the list of terms produced by the symbolic execution of $\Pi_{1}$ and $\Pi_{2}$. Hence the security formula obtained this way

must be derived as a logical consequence of the axioms $A$ using first-order inference. If the formula is derived and the axioms in $A$ shown to be computationally sound, then the protocol $\Pi$ is computationally secure. If the security formula cannot be derived, then the negation of the security formula is consistent with $A$ and a symbolic attacker model is then obtained. In practice, for the security proof, a proof tree is being built, and some branch cannot be reduced to axioms. For example, often what happens is that it cannot be shown that an attacker message $f_{i}(\cdots)$ cannot equal a term, that is, the attacker may produce a message that passes some check when it should not. Then from this branch, an Herbrand model can be built on the terms, resulting in a symbolic attack. This symbolic attack then has to be checked to see if it corresponds to a computational attacker or if it originates from having too few axioms in $A$, as the set of axioms is not complete.

\subsection{Our Contributions}

\subsubsection{Analyzing FOO voting protocol using CCSA}

The main contribution of this work is to analyze the electronic voting protocol introduced by Fujioka, Okamoto, and Ohta in [FOO93], which we call it FOO protocol, in the provable security model rigorously, using the BC technique of Indistinguishability properties. As far as we know, this is the first formal analysis of the FOO protocol in the provable security model. Besides proving vote privacy for FOO, our other aim is to develop the library of axioms of the CCSA framework further and to demonstrate its effectiveness in attack detection and verification. 
Attacks. Our analysis revealed new attacks on the FOO protocol (see Section 3.4.2).

The first attack occurs because of an inadequacy of blindness property of blind signatures. Intuitively, blindness [JLO97] means that a dishonest signer who engages in two sessions (parallel or sequential) with an honest user on messages $m_{0}$ and $m_{1}$ cannot detect which session is for $m_{0}$ and which session is for $m_{1}$ if the user successfully outputs signatures in both sessions. The blindness property, however, does allow the possibility that the attacker can distinguish between the sessions if the user is successful in only one session. In order to prevent this attack, we have to assume that the identities of the candidates are of equal length. This attack does not depend on the implementation of the anonymous channel.

The second attack exploits the fact that encryption scheme used by the mix-net server may be length-revealing and hence the length of the encrypted messages to the mix-net server may reveal their senders. In order to prevent this, we have to assume that the signatures obtained on equal length messages by executing the blind signature interactive protocol with the same signer must be of equal length. The above two attacks lie outside the DY model and hence were not detected in previous works on formal analysis of FOO protocol.

A third attack is a DY style replay attack in which messages from the Voting phase can be replayed in the Opening phase. This attack can be prevented by introducing phase numbers in the FOO protocol.

Finally, we point out a man-in-the-middle attack in which a dishonest voter intercepts one of the two ballots of the honest voters and sends some garbage to the mixer instead. Then in the opening phase, the attacker creates a bulletin board which contains all the ballots of the honest voters. Unaware to this situation, the honest voters follow the protocol and reveals their commitment keys in the opening phase. This attack can be prevented by generating and including a nonce in the ballots of honest voters in the voting phase. 
With these additional assumptions, we establish vote privacy of the FOO protocol for one session with two honest voters and one dishonest voter. The proof carries over to $n$ dishonest voters for any fixed $n$. The proof of vote privacy rests on the blindness property of the blind signature, the computational hiding and binding properties of the commitments and of IND-CCA2 assumption on the encryptionused in the anonymizing mechanism. The proof of vote privacy in the DY model, in contrast, relies only on the blindness property. ${ }^{1}$ The commitment hiding property of trapdoor commitments does not play a role in establishing vote privacy in the DY analysis.

\subsubsection{Mechanizing the proofs using Coq}

Our other contribution, noting the fact that the handwritten proofs are error-prone and to achieve higher assurances, is to formalize the proofs of vote privacy of the FOO voting protocol in the Coq proof assistant [BC04, $\left.\mathrm{PdAC}^{+} 17\right]$

Towards this, we have formalized the syntax and the axiomatic system in Coq, and utilized the axiomatization to obtain machine-checked proofs of vote privacy of the FOO protocol in Coq. We demonstrate the effectiveness of our mechanization with the verification of authentication and secrecy guarantees of the Authenticated Diffie-Hellman key exchange protocol which is a simplified version of Station-ToStation (STS) protocol - the handwritten proofs originally appear in the publication [BCEO19] and reproduced in the section 5.3 of Chapter 5. For the FOO and STS protocol, we consider only one session each of responder and initiator in order to keep the formulas small. All the machine-checked proofs are available at [Eer19].

Although the mechanized proofs look simpler and straight forward, the process of building the CCSA framework in Coq is tedious and complex for two reasons. First, due to the challenge posed by the equality predicate $=$, which is, an abbreviation constructed from $\sim$, defined between terms. = represents equality (not identity) that

\footnotetext{
${ }^{1}$ As evidence, we ran AKiSs [CCCK16] on a two-phase variant of the FOO protocol without commitments. The variant satisfies the vote privacy property in the DY model.
} 
can fail with negligible probability and serves as a congruence in the logic. The native equality relation of $\mathrm{Coq}$ is Leibnizian identity [BC04, $\left.\mathrm{PdAC}^{+} 17\right]$, which forces two terms of our syntax to be equal (in the sense of native Coq equality relation) if and only if they are syntactically identical. This assumption would be unsound for our semantics, where syntactic difference does not necessarily entail inequality. Hence, we define our equality relation and use morphisms [BC04, $\left.\mathrm{PdAC}^{+} 17\right]$ to model the relation as a congruence relation. This allowed us to reuse native Coq tactics such as rewrite and replace seamlessly. Second, due to the complexity arise from mutual recursiveness and nested behavior of the types message and bool. In such a scenario, Coq generates a weak induction, which is not strong enough to prove properties about the types. As explained in Section 3.8 of [Chl13], we strengthen the induction manually and use it in place of the automatically generated one.

\subsection{Related Works}

There have been several attempts at formal analysis of FOO protocol in the Dolev-Yao model (see, for example, [KR05, DRS08, CCCK16]). These analyses assume perfectly anonymous channels. In the computational model, there are several attempts at formalizing vote privacy in electronic voting, such as in [BT94, Ben87, SB13, BCG $\left.{ }^{+} 15\right]$. Please see $\left[\mathrm{BCG}^{+} 15\right]$ for comparison amongst these definitions. All these definitions apply to single-phase voting protocols. Our definition is adapted to FOO-protocol, which has three phases. The only other work at formally verifying vote privacy for electronic voting that we are aware of is the mechanized proof of vote privacy for the Helios family of single-phase protocols given in $\left[\mathrm{CDD}^{+} 17\right]$. The inadequacy of the blindness axiom has also been pointed out in [FS09], who show how any blind signature scheme can be combined with commitments to resolve this inadequacy.

CCSA technique was first introduced by Bana and Comon in [BCL12] for reach- 
ability properties and then for indistinguishability properties in [BCL14]. Since then it has been used to find new attacks to the Needham-Schroeder-Lowe protocol [BAS12a, BAS12b]; to treat algebraic operations notoriously difficult to reason about in the DY model, such as exponentiation along with it the decisional Diffie-Hellmann property and versions of the Diffie-Hellman key-exchange protocol [BC16, BCEO19]; to verify unlinkability of RFID protocols [CK17]; and to analyze key wrapping API's [SS16]. Automated tool is not yet available for the indistinguishability technique, but work is in progress (see [Kou19]). In the meantime, we continue developing the library of axioms, and verifying relatively simple protocols by hand, and with Coq.

Note that some of the results of our contributions appear in the conference paper [BCE18] and in the journal publication [BCEO19].

\subsection{Outline of the Dissertation}

We present the outline of the thesis. In Chapter 2, we recall the state-art-of-the-art attacker models in protocol analysis such as Dolev-Yao and computational. We recall the approaches of establishing computational guarantees of the symbolic analysis. In particular, we briefly recall the CCSA framework and the verification methods using the model. We also discuss their strengths and weaknesses. The formalization of the FOO protocol and its analysis using the CCSA framework for equivalence properties is presented in the Chapter 3. This includes the FOO protocol, the core axioms of the $\mathrm{BC}$ technique, the axioms that model the cryptographic hypothesis, and the transition systems that simulate the protocol. The mechanized proofs of vote privacy of the FOO voting protocol - the formalization of equality, induction, authentication

and secrecy of the Authenticated Diffie-Hellman key exchange protocol presented in the Chapter 5. Finally, the conclusion and future work presented in Chapter 6 . 


\section{Chapter 2}

\section{Background}

Security protocols are distributed programs that are aimed at ensuring properties such as confidentiality, authentication, privacy, and so forth. The protocols are ubiquitous in modern-day communication. However, it is not uncommon to have flaws in them. For example, consider the protocol developed by Roger Needham and Michael Schroeder [NS78] in 1978. The protocol is supposed to achieve mutualauthentication - both the parties in the protocol should agree on some secret valuesof the two parties of the protocol on successful completion. The protocol is described in Figure 2.1

\begin{tabular}{|c|c|c|}
\hline Alice (Initiator) & & Bob (Responder) \\
\hline 1: & $\left\{A, n_{A}\right\}_{p k_{B}}$ & \\
\hline $2:$ & $\left\{n_{A}, n_{B}\right\}_{p k_{A}}$ & \\
\hline 3: & $\left\{n_{B}\right\}_{p k_{B}}$ & \\
\hline
\end{tabular}

Figure 2.1: Needham-Schroeder protocol

In Figure 2.1, $\{X\}_{p k_{Y}}$ represents encryption of the message $X$ with the public-key of 
the agent $Y$. At the end of the protocol, the agents $A$ and $B$ should agree on the random values of $n_{A}$ and $n_{B}$. The protocol is believed to be correct for about 17 years. However, in 1995, Gavin Lowe [Low95] showed that the protocol is vulnerable to man-in-the-middle-attack. It is presented below.

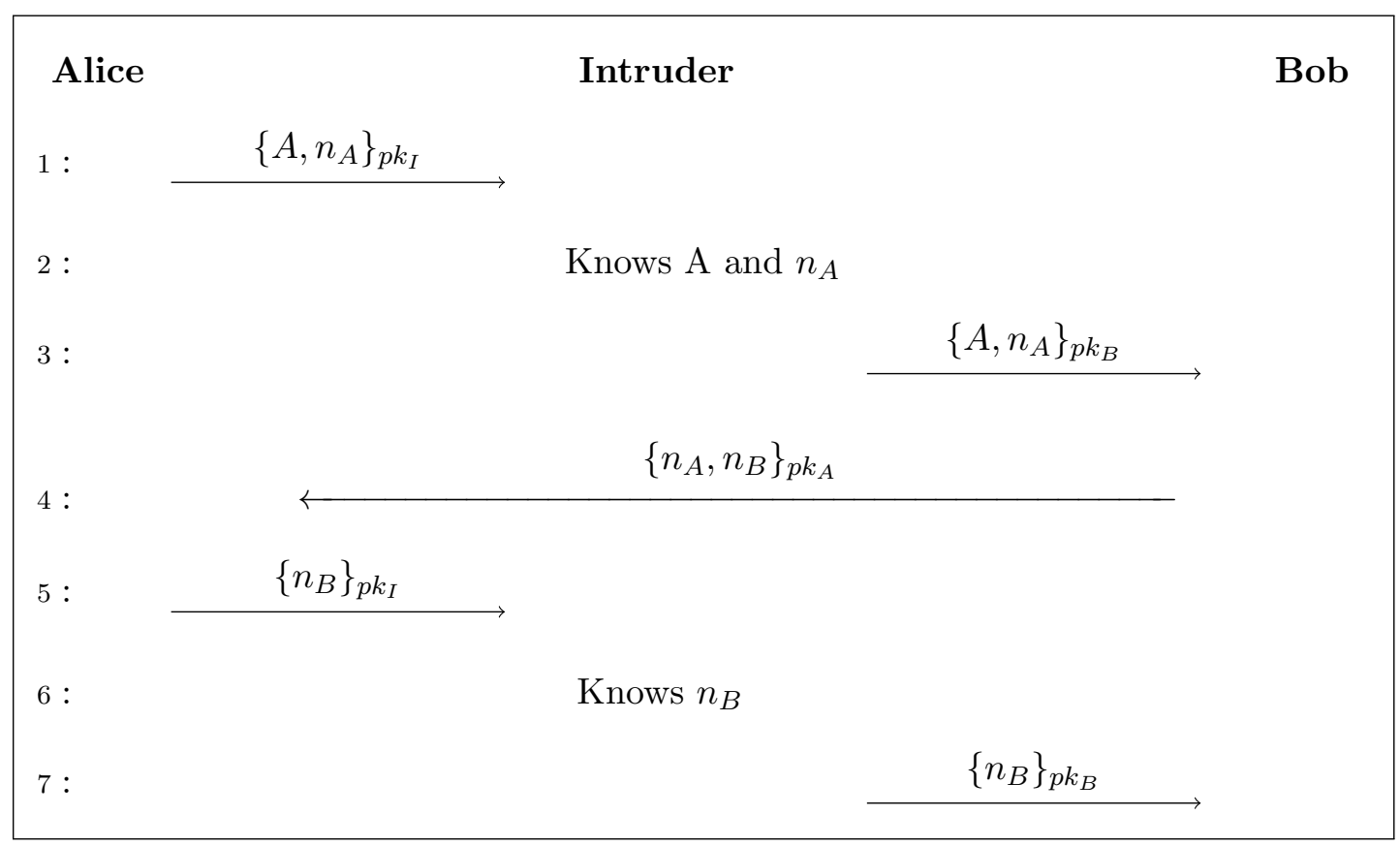

Figure 2.2: Man-in-the-middle attack on NS protocol

The agent $A$ believes that he is communicating with the agent $I$ while the agent $B$ believes to be in engaging with $A$. However, the malicious agent $I$ impersonates $B$ as $A$ and learns the secrets $n_{A}$ and $n_{B}$.

Lowe [Low96] also proposed a fix and showed that the same attack could not be mounted on the protocol. 


\begin{tabular}{|ll|}
\hline \multicolumn{1}{|c|}{ Alice (Initiator) } & Bob (Responder) \\
\hline $1:$ & $\frac{\left\{A, n_{A}\right\}_{p k_{B}}}{}$ \\
$2:$ & $\left\{B, n_{A}, n_{B}\right\}_{p k_{A}}$ \\
$3:$ & $\left\{n_{B}\right\}_{p k_{B}}$ \\
\hline
\end{tabular}

Figure 2.3: Needham-Schroeder-Lowe protocol

By including $B$ 's identity in the message, one can avoid the attack.

In order to have higher confidence in the use of the security protocols, one should analyze them rigorously by modeling capabilities of an attacker. There are two wellknown worlds in formalizing the attacker capabilities, namely, symbolic model and computational model. These two models have been evolving independently over a period of 20 years. However, each framework has its own advantages and drawbacks, and we will try to unfold them in the subsequent sections.

\subsection{The Symbolic Model}

This framework has been developed by Danny Dolev and Andrew Chi-Chih Yao in 1983 and is described in the seminal paper [DY83]. This framework often goes by other names such as Dolev-Yao or formal model. In this model, the messages are abstract-terms that are built using the term-algebra. The attacker is allowed to perform some operations include building terms by applying the function symbols of the protocol. These operations include encryptions, projections of a pair, commitment creation, and so forth. The operations are explicitly specified as inference rules, and the attacker is allowed to draw a conclusion by applying only these rules. A few examples of such rules are presented below. 


$$
\frac{x y}{\langle x, y\rangle} \quad \frac{m k}{\operatorname{enc}(m, k)} \quad \frac{m k}{\operatorname{com}(m, k)}
$$

However, one should be careful in specifying these inference rules. The rules may directly result from the properties of cryptographic primitives. For example, consider the commitment schemes that satisfy the property open $(\operatorname{com}(x, y), y)=x$. Unless we specify this property explicitly as an inference rule, the attacker is not able to open the commitments. Hence, the model will lose an attack that makes use of this property. The security properties in this model are categorized into two: reachability properties and equivalence properties. The widely known reachability properties are confidentiality (secrecy) and authentication. Informally, the secrecy of value can be achieved if an adversary is not able to compute the value during the protocol. Usually, the equivalence based properties are achieved by reducing them to the indistinguishability of two processes. Examples of these properties, including vote privacy [KR05], which is the main topic of this proposal, states that an adversary can not distinguish two processes when the two votes are swapped. In this model, the proofs are easy to automate. This is because the attacker's capabilities are specified in relatively simple logic as inference rules. In fact, there are numerous automated tools available for verifying the security properties in this framework, namely, ProVERIF [Bla05], Avispa [ea05], Scyther [Cre08], Akiss [CCK12], SAT-Equiv [CDD18], and so forth.

\subsection{The Computational Model}

On the other hand, the messages in the computational model are not abstract terms but the bit-strings - strings of 0's and 1's. The computational attacker is modeled as a Probabilistic Polynomial-time Turing (PPT) machine whose inputs and the output are bit-strings. This framework has been developed by Goldwasser, Micali, Rivest, 
and Yao. The interested reader is referred to the initial papers [Gol84, GMR88, Yao82]. This model leverages complexity and probability theory to express the security properties and the cryptographic assumption precisely. In general, these properties are described as a game between the attacker and the challenger such that the adversary can not win the game. For example, consider the property of computational hiding of the commitment schemes defined in Section 3.3. The experiment $\operatorname{Hiding}_{\mathcal{A}}^{\mathcal{C}}$ is explained as follows. Firstly, the adversary picks two messages, $m_{0}$ and $m_{1}$ of the same length, and passes them to the challenger. The challenger uniformly chooses a bit $b$ from $\{0,1\}$, and honestly generates two keys $k_{0}$ and $k_{1}$. Then the challenger computes $\operatorname{com}\left(m_{b}, k_{0}\right)$ and $\operatorname{com}\left(m_{1-b}, k_{1}\right)$ and sends out to the adversary. Finally, the adversary has to output a guess for $b$. In order to achieve the property of computational hiding, the attacker should not win the game with non-negligible probability. Similarly, the property of computational secrecy has been defined. Informally, secrecy states that an attacker can not distinguish the secret value from a random number given by an oracle. In general, the security proofs in this framework are merely reductions to some hardness assumptions about the cryptographic primitives. In other words, the probability of successfully breaking a security property of a protocol bounds by the probability of breaking its primitives.

The advantages of this model include the precise modeling of an attacker as well as the assumptions of the cryptographic primitives. However, it is not easy to find minimal hardness assumptions the security proof can reduce to. Moreover, it is desirable to have assisted by a machine, if not, at least partly. In fact, there are two proof assistants, CRYptoVERIF and EAsyCRYPt, available for verifying the properties of the security protocols in the computational model. The tools are briefly recalled below.

CryptoVerif. This tool has been developed by Bruno Blanchet and presented in [Bla08b]. It is based upon the probabilistic PT process calculus introduced in [MRST01] 
and the game hopping method, which has been introduced by Shoup et al. (see [Sho04]). It produces proofs of sequences of games by applying definitions of the assumptions of the cryptographic primitives. The initial game is supposed to be the original game, and the final game is the one from which the security property is obvious. The tool also outputs an upper bound on the probability of a successful attack. CryptoVerif has been successful in verifying deducibility properties such as secrecy and authentication. However, the tool has several limitations. For example, the prover fails to prove the confidentiality of the Needham-Schroeder public-key protocol when the exchanged key is the nonce $n_{a}$ even though the property holds.

EasyCrypt. This tool is due to Barthe et al [BGHZB11]. EasyCrypt is also based on the game hoping method and proofs for sequences of games. However, the tool has only been largely used to verify security properties of the primitives such as encryption schemes, digital signatures, hash functions, and so forth.

Notice that instead of specifying what an attacker can do - as the symbolic model does - the computational framework specifies what an attacker is not allowed to do. These restrictions are stems from the assumptions of the cryptographic primitives. These properties are defined as precisely as possible in this framework. Moreover, messages are bit-strings as what the real messages look like. Hence, the computational model facilitates to reason about the properties of the security protocols rigorously than the Dolev-Yao model.

However, the computational model has several limitations. For example, the provers of this framework need human interaction. Moreover, it is not clear when a prover fails to complete a proof-Is it due to the limitations of the prover? Is the security property is not satisfied by the protocol? Or Is it the usability issue? Even if a prover achieved proof, it is hard to read and understand.

With keeping these issues in mind, Abadi and Rogaway introduced an approach [AR02], which aims at relating these two worlds - the computational and the symbolic model. 
By noting the fact that the Dolev-Yao model is amenable to automation while the computational analysis allows one to precise reason about the protocols, the authors introduced a way to establish computational guarantees of the symbolic analysis. This is achieved by making the symbolic attacker as powerful as the computational one. In other words, by making sure, for every computational attack, there is a corresponding symbolic attack. The research in this direction was quite successful for some years (see, for example, [BP04, BPW03, CLC08b, MW04]). However, their assumptions about the cryptographic primitives are disputable. One can argue that assumptions can never be implemented in the real world.

\subsection{Computational Soundness}

Under certain conditions, the Dolve-Yao model is fully abstract with respect to computational one, and analysis of protocols in the former model guarantees computational proofs. The readers are directed to the initial papers [AR02, BPW03] in this direction. However, this approach has several limitations. The proofs of establishing computational guarantees rely upon very strong assumptions of the computational implementation and are often unrealistic. Moreover, the soundness results are not modular-adding additional primitive requires to establish the soundness results for the whole system again. The proofs of computational soundness results are very long and complex. Due to these issues, the research in this direction has largely been abandoned by now - although once it has been received much attention. Currently, the researchers are mostly focusing on developing tools that carry the proofs directly in the provable security model. 


\subsection{Computationally Complete Symbolic Attacker}

In order to overcome the discrepancy as mentioned above, Bana and Comon proposed a new approach(see [BCL12]) that aimed at establishing computational guarantees while leveraging the symbolic techniques with reasonable assumptions about the cryptographic primitives. The authors named it as Computationally Complete Symbolic Attacker (CCSA). Although the framework has only been used for verifying reachability properties initially [BAS12a, BHO13], with [BCL14, Sce15], the creators demonstrated the practicality of the framework in the verification of equivalence properties. We reproduce the background of the model from [BCE18].

The CCSA framework is similar to the Dolev-Yao framework in the sense that explicit symbolic adversarial messages are created. The Dolev-Yao framework specifies all rules that the attacker can use to create new messages from what he has seen, and the protocol agents use pattern matching to check whether messages coming from the attacker have the correct form. On the other hand, in the CCSA framework, each message from the attacker is modeled by a function $f_{i}$ applied to the sequence of messages that the attacker has seen thus far. Pattern matching is replaced by applying function symbols on the term coming from the attacker. Limitations on attacker capabilities originating from computational assumptions on the primitives are specified as a set of axioms $A$ in first-order logic based on a single indistinguishability predicate (representing computational indistinguishability of sequences of messages). Computationally, a security property of a protocol $\Pi$ is formulated as the computational indistinguishability of two protocols $\Pi_{1}$ and $\Pi_{2}$ constructed (depending on the security property) from the original $\Pi$.

Verification. In the CCSA framework, the security translates to the validity of the formula obtained by applying the indistinguishability predicate on the list of terms produced by the symbolic execution of $\Pi_{1}$ and $\Pi_{2}$. Hence the security formula obtained this way must be derived as a logical consequence of the axioms $A$ using 
first-order inference. If the formula is derived and the axioms in $A$ shown to be computationally sound, then the protocol $\Pi$ is computationally secure according to Theorem 1 in [BCL14].

Attack finding. If the security formula cannot be derived, then the negation of the security formula is consistent with $A$ and a symbolic attacker model is then obtained. In practice, for the security proof, a proof tree is being built, and some branch cannot be reduced to axioms. For example, often what happens is that it cannot be shown that an attacker message $f_{i}(\cdots)$ cannot equal a term, that is, the attacker may produce a message that passes some check when it should not. Then from this branch, an Herbrand model can be built on the terms, resulting in a symbolic attack. This symbolic attack then has to be checked to see if it corresponds to a computational attacker or if it originates from having too few axioms in $A$, as the set of axioms is not complete.

\subsubsection{Syntax}

Terms: Let $S$ be a finite set of sorts that includes at least the sorts bool and message. $\mathcal{X}$ is an infinite set of variable symbols, each coming with a sort $s \in S$. Terms are built on a set of function symbols $\mathcal{F}$ representing honest computation of primitives, a set of function symbols $\mathcal{G}$ representing attacker's computation, a set of zero-arity function symbols (names) $\mathcal{N}$ representing honest generation of randomness, and a set of variables $\mathcal{X}$. The set $\mathcal{F}$ contains the basic symbols such as $\mathbf{0}$, true, false, $\mathrm{L},\left\langle_{-},{ }_{-}\right\rangle$, $\mathrm{EQ}\left({ }_{-},{ }_{-}\right)$, if _ then _ else $\_, \mathrm{z}, \mathrm{compl}, \pi_{1}$ and $\pi_{2}$ with the typing rules as follows:

- 0 : message representing the empty message.

- true, false : bool.

- $\mathrm{L}(-)$ : message $\rightarrow$ message. $\mathrm{L}(x)$ represents the length of $x$ in unary.

- $\left\langle{ }_{-},{ }_{-}\right\rangle$: message $\times$message $\rightarrow$ message representing pairs. 
- Equality test $\mathrm{EQ}\left({ }_{-}\right)$. message $\times$message $\rightarrow$ bool

- Equality test EQ(-, $\left.{ }_{-}\right)$:

bool $\times$ bool $\rightarrow$ bool.

- Conditional branching if_then_else ${ }_{-}$: bool $\times$message $\times$message $\rightarrow$ message bool $\times$ bool $\times$ bool $\rightarrow$ bool.

- $\mathrm{z}\left({ }_{-}\right)$: message $\rightarrow$ message. $\mathrm{z}(x)$ represents the string of 0's whose length is equal to the length of $x$.

- $\operatorname{compl}\left({ }_{-}\right):$message $\rightarrow$ message. $\operatorname{compl}(x)$ represents the message which is complement of $x$.

- $\pi_{1}\left({ }_{-}\right), \pi_{2}(-)$ : message $\rightarrow$ message. $\pi_{i}$ represents the $i$-th projection of a pair

Formulas: As presented in [BC16], we have for every sequence of sorts $s_{1}, \ldots, s_{n}$ a predicate symbol that takes $2 \times n$ arguments of sort $\left(s_{1} \times \ldots \times s_{n}\right)^{2}$, which we write as $t_{1}, \ldots, t_{n} \sim u_{1}, \ldots, u_{n}$. The predicate $t_{1}, \ldots, t_{n} \sim u_{1}, \ldots, u_{n}$ represents computational indistinguishability of the two sequences of terms $t_{1}, \ldots, t_{n}$ and $u_{1}, \ldots, u_{n}$.

The first-order formulas are built from the above atomic formulas combining the Boolean connectives $\neg, \wedge, \vee$, and $\rightarrow$, and the quantifiers $\forall$ and $\exists$. The formulas are used to represent both axioms (assumptions) and the security properties of the protocols.

Equational Theory: We use binary relation symbol $=_{E}$ to indicate equations the function symbols have to satisfy. Note that $={ }_{E}$ is not part of the first-order signature. The equations specified by $=_{E}$ will result in axioms for $\sim$. We assume that the following hold:

- $\forall x_{1}, x_{2} \cdot \pi_{i}\left(\left\langle x_{1}, x_{2}\right\rangle\right)={ }_{E} x_{i}$, for $i=1,2$

- $\mathrm{L}(\mathrm{L}(x))={ }_{E} \mathrm{~L}(x)$.

Abbreviations: In order to reduce the size of the terms, we shall use the following abbreviations: 
- $\operatorname{not}(b) \stackrel{\text { def }}{\equiv}$ if $b$ then false else true

- $b_{1} \& b_{2} \stackrel{\text { def }}{\equiv}$ if $b_{1}$ then $b_{2}$ else false

- $b_{1}$ or $b_{2} \stackrel{\text { def }}{\equiv}$ if $b_{1}$ then true else $b_{2}$

- $x=y \stackrel{\text { def }}{\equiv} \mathrm{EQ}(x, y) \sim$ true

- $\operatorname{EQL}\left(x_{1}, x_{2}\right) \stackrel{\text { def }}{\equiv} \mathrm{EQ}\left(\mathrm{L}\left(x_{1}\right),\left(\mathrm{L}\left(x_{2}\right)\right)\right)$

\subsubsection{Semantics}

As Bana and Comon defined it in [BCL14], the logic is interpreted over a computational model. A computational model $\mathcal{M}^{c}$ is a particular first-order model in which the domain consists of probabilistic polynomial-time algorithms taking the input $1^{\eta}$ together with two infinitely long random tapes $\left(\rho_{1}, \rho_{2}\right)$, where $\rho_{1}$ is for honestly gener-

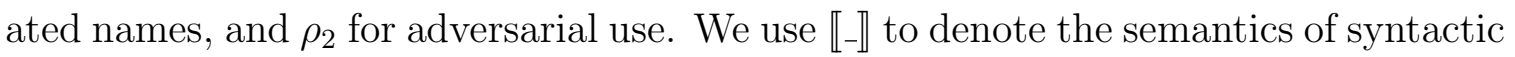
objects. Once $\llbracket-\rrbracket$ is given on function symbols, and a valuation $\sigma$ of variables of term $t$ in the domain, the interpretation of $t$ in the domain is defined the usual way and denoted as $\llbracket t \rrbracket^{\sigma}$. Let $\llbracket t \rrbracket_{\eta, \rho}^{\sigma}:=\llbracket t \rrbracket^{\sigma}\left(1^{\eta} ; \rho_{1}, \rho_{2}\right)$. In this model, function symbols in $\mathcal{F}$ representing the cryptographic primitives are interpreted as polynomial-time algorithms and they act on the outputs of the PPT algorithms in their arguments. For example, $\llbracket \mathrm{EQ}\left(t_{1}, t_{2}\right) \rrbracket_{\eta, \rho}^{\sigma}:=1$ if $\llbracket t_{1} \rrbracket_{\eta, \rho}^{\sigma}=\llbracket t_{2} \rrbracket_{\eta, \rho}^{\sigma}$, otherwise it is 0 , and $\llbracket$ if $t_{1}$ thent $t_{2}$ elset $\rrbracket_{3} \rrbracket_{\eta, \rho}^{\sigma}:=\llbracket t_{2} \rrbracket_{\eta, \rho}^{\sigma}$ if $\llbracket t_{1} \rrbracket_{\eta, \rho}^{\sigma}=1$, and $\llbracket$ if $t_{1}$ then $t_{2}$ else $t_{3} \rrbracket_{\eta, \rho}^{\sigma}:=\llbracket t_{3} \rrbracket_{\eta, \rho}^{\sigma}$ if $\llbracket t_{1} \rrbracket_{\eta, \rho}^{\sigma}=0$. $\llbracket z(t)_{\eta, \rho}^{\sigma} \rrbracket=0^{|t|}$, where $|t|$ denotes the length of the term $t . \llbracket \operatorname{compl}(t)_{\eta, \rho}^{\sigma} \rrbracket=t^{\prime}$, where $t^{\prime}$ is a complement of $t$ such that $t \oplus t^{\prime} \neq 0$. Function symbols in $\mathcal{G}$ representing adversarial computation are interpreted as probabilistic polynomial-time algorithms and they also act on the outputs of the PPT algorithms in their arguments, but they can also use randomness from $\rho_{2}$. Each name $n \in \mathcal{N}$ is interpreted as a machine $\llbracket n \rrbracket$ which extracts a random word of length $p(\eta)$ (where $p$ is a polynomial globally fixed by $\mathcal{M}^{c}$ ) from $\rho_{1}$. Different 
names draw from disjoint parts of the tape $\rho_{1}$. The predicate symbol $\sim$ is interpreted as computational indistinguishability of the outputs of the PPT algorithms on the two sides of $\sim$. Interested reader can consult either [BCL14] or [BC16] for complete definition of this semantics. A formula is computationally valid if it is satisfied in all computational interpretations.

\subsubsection{Protocols}

With the authors' permission, we borrowed the content of this Section from [BC16].

The authors of [BCL14] treated protocols as abstract transition systems without committing to any particular way of specifying protocols. They could be specified for instance in the applied pi-calculus [AF01] or any other process calculus. The authors of [BCL14] also assumed a bounded number of sessions: each protocol comes with an arbitrary but fixed bound on the number of steps in its execution. It would be possible to define the protocols without such a bound, but the general soundness result (Theorem 1 of [BCL14]) holds only for computational adversaries that exploit bounded number of sessions in the security parameter. Therefore, without loss of generality we can just as well put the bound in the protocol for simplifying the formulation.

\subsubsection{The transition system}

We shall now introduce the abstract transitions systems used in [BCL14]. Observe that in our transition systems, we shall also decorate the states with the names generated in the transition. A protocol is an abstract transition system defined by:

- A finite set of control states $Q$ with a strict partial ordering >, an initial state $q_{0}$ and a set $Q_{f} \subseteq Q$ of final states. 
- For each state $q \in Q$, a linearly ordered (finite) set $T(q)$ of transition rules

$$
q,\left(N_{0}, N_{1}, \ldots, N_{n}\right),(\vec{x}) \stackrel{\theta}{\rightarrow} q^{\prime},\left(N_{0}, N_{1}, \ldots, N_{n}, N\right), s,(\vec{x}, x)
$$

$-\vec{x} \equiv x_{1}, \ldots, x_{n}$ and $x$ are variables.

$-N_{0}, N_{1}, \ldots, N_{n}, N$ are lists of names.

- $\theta$ is a term of sort bool with variables in $x_{1}, \ldots, x_{n}, x$

- $q, q^{\prime} \in Q$ are such that $q>q^{\prime}$.

$-s$ is a term with variables in $x_{1}, \ldots, x_{n}, x$.

$T(q)$ is empty if and only if $q \in Q_{f}$. Otherwise, $T(q)$ contains a maximal transition, whose guard $\theta$ is true.

- An initial knowledge $\phi_{0}$.

Intuitively, a transition $q,\left(N_{0}, N_{1}, \ldots, N_{n}\right),(\vec{x}) \stackrel{\theta}{\rightarrow} q^{\prime},\left(N_{0}, N_{1}, \ldots, N_{n}, N\right), s$, $(\vec{x}, x)$ is a guarded transition which changes the state from $q$ to $q^{\prime}$ upon receiving the message $x$; the variables $x_{1}, \ldots x_{n}$ store the messages sent by the attacker so far, $N_{i}$ is the list of names generated upon the receipt of $x_{i}$, and the Boolean condition $\theta$ specifies the condition under which the transition can be fired (namely, the conditions under which a participating agent moves forward). The term $s$ specifies the message being sent out in the transition, that is, the message sent by the agent with new names in $N$. The partial ordering on states ensures progress and hence termination. The linear ordering on transitions specifies in which order the guards have to be tried. The ordering on the states thus rules out any non-determinism in the protocol itself.

Example 2.1 The Diffie-Hellman key exchange protocol is the following (see e.g. [Bon98]):

- A group description $G$ and a group generator element $g$ are generated honestly, according to a randomized algorithm, and made public. 
- The Initiator generates a random $a$ in $\mathbb{Z}_{|g|}$ and sends $g^{a}$.

- The Responder receives $g^{a}$, generates a random $b$ in $\mathbb{Z}_{|g|}$ and sends $g^{b}$, and computes $\left(g^{a}\right)^{b}$.

- The Initiator receives $g^{b}$, and computes $\left(g^{b}\right)^{a}$.

\begin{tabular}{|lll|}
\hline Alice (Initiator) & Bob (Responder) \\
\hline$a \leftarrow s \mathbb{Z}_{|g|}$ & & \\
$X \leftarrow g^{a}$ & & \\
& & \\
& & \\
& & \\
& & \\
& & \\
& & \\
& & \\
& & $g^{b}, g,|g|, g^{a} \mathbb{Z}_{|g|}$ \\
$\mathrm{k}_{A} \leftarrow Y^{a}$ & & \\
& & \\
\hline
\end{tabular}

Figure 2.4: Diffie-Hellman key exchange protocol

Here we shall consider two honest parties running one parallel session, each of which may be initiator and responder. More sessions can be analyzed similarly: the terms would be much bigger, but there would not be any qualitative difference. As mentioned earlier, the protocol formulation of [BCL14] rules out any non-determinism. We assume that the message coming from the adversary specifies which session the agent should assign it to. Note, the adversary can, of course, direct messages to incorrect sessions thereby creating confusion.

Accordingly, since we want to consider one session for each participant, we introduce two session identifiers (message constants in $\mathcal{F}$ ): one for agent $A$ : $\mathrm{id}_{1}$, and one for agent $B: \mathrm{id}_{2}$. We further introduce a function symbol to : message $\rightarrow$ message 
which extracts from an incoming message the session. As for their semantics, the session identifiers can be any fixed, distinct bit strings and to is a function that extracts from a bit string a part that is agreed to be the position for the session identifier. On a bit string that is of the wrong form, the interpretation of to can give an error. We also assume that the session identifiers are distinct: $\mathrm{EQ}\left(\mathrm{id}_{\alpha}, \mathrm{id}_{\beta}\right) \sim$ false for sessions $\alpha \not \equiv \beta$. Here we use $\alpha, \beta$ to denote any of 1,2 . Finally, to ensure that the Initiator also responds something at the end of its role so that execution of other sessions can continue, we introduce an accept message acc : message.

Then the initiator role of $A$ for session $\mathrm{id}_{\alpha}$ is the following:

- $A$ receives a message into $x_{1}$.

- If to $\left(x_{1}\right)=\mathrm{id}_{\alpha}$, and $x_{1}$ instructs $A$ to start a new session, then $A$ generates an $a$ in $\mathbb{Z}_{|g|}$, and sends $g^{a}$.

- $A$ receives message into $x_{2}$.

- If to $\left(x_{2}\right)=\mathrm{id}_{\alpha}$, then $A$ computes $\mathrm{m}\left(x_{2}\right)^{a}$ and sends acc.

The responder role of $A$ is the following:

- $A$ receives a message into $y_{1}$.

- If to $\left(y_{1}\right)=\operatorname{id}_{\alpha}$, then $A$ generates an $a$ in $\mathbb{Z}_{|g|}$, computes $\mathrm{m}\left(y_{1}\right)^{a}$ and sends $g^{a}$.

In order to make notation more accessible, we present a transition diagram that represents the DH key exchange protocol with an initiator session $i d_{1}$ and a responder session $\mathrm{id}_{2}$ played by the agents $A$ and $B$ respectively in Figure 2.5. As expected, a state $q_{\ell_{1}, \ell_{2}}^{k_{1}, k_{2}}$ will represent the state of each agent $A$ and $B$. If $A$ ( $B$ respectively) is an initiator then $k_{1}\left(k_{2}\right.$ respectively) will be 1 if waiting for a response and 2 otherwise. If $A$ ( $B$ respectively) is a responder then $\ell_{1}\left(\ell_{2}\right.$ respectively) will be 1 . The diagram illustrates three possible branches of the $\mathrm{DH}$ protocol. The right-most branch of $q_{00}^{00}$ simulates the situation where Aacts as an initiator and $B$ plays the responder role whereas the left-most branch simulates the other scenario where Bplays the initiator role and $A$ plays the responder role. The middle branch illustrates the scenario when 


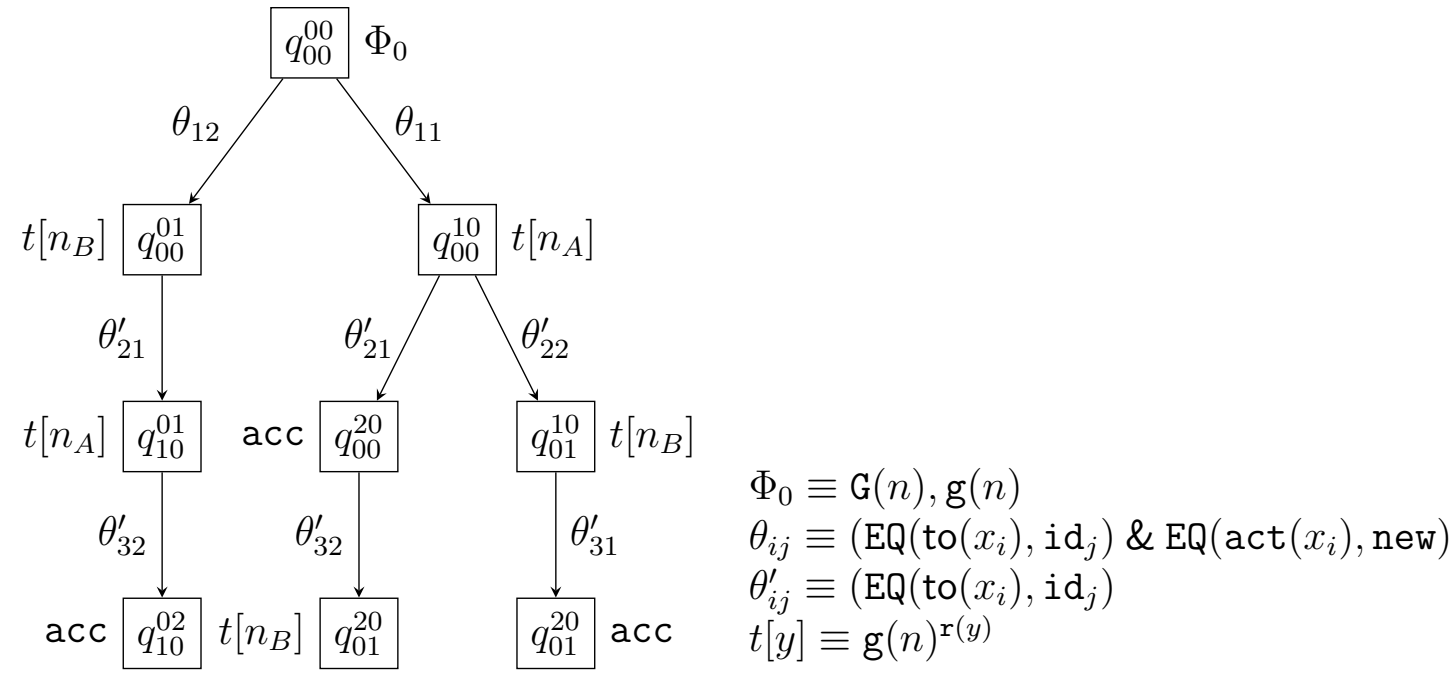

Figure 2.5: DH key exchange protocol with an initiator session and a responder session all the initiator moves of $A$ happen before the responder moves of $\mathrm{B}$. At the initial state, the honestly generated description of a cyclic group $\mathrm{G}(n)$ and a generator of the group $\mathrm{g}(n)$ are generated honestly and made available to the attacker. This initial knowledge of the attacker is represented by the frame $\Phi_{0} . \theta_{i j}$ s represent the conditions that the agent checks if he was instructed to start a new session upon receiving the message $x_{i}$ in the session $i \mathrm{~d}_{j}$ while $\theta_{i j}^{\prime}$ s are the conditions that the agent checks if the message $x_{i}$ is for the session $i \mathrm{~d}_{j}$. The output term $t[y]$ represents the group exponent computed by an agent $y$ in the protocol. Of course, at the end of the protocol, the initiator also sends out the message acc to indicate that the protocol has been completed.

\subsubsection{Executions and indistinguishability}

Computational and symbolic executions were defined precisely in [BCL14]. Instead of repeating the abstract definitions here, we appeal to the reader's intuition, and only illustrate through examples how the executions work symbolically as we carry out the proof there.

We recall first that in case of the symbolic execution, to treat protocol indistinguishability of protocols $\Pi$ and $\Pi^{\prime}$, the Dolev-Yao way would be to match the branches 
of the execution of $\Pi$ and that of $\Pi^{\prime}$ such that the matched branches are statically equivalent (see e.g. [CLC08a]).However, as the authors discussed in [BCL14], obtaining computational soundness through such matching seems infeasible. Instead, the authors in [BCL14] folded the protocol execution into a single trace, such that the tests of the participating agents at each round on the incoming message were included in the terms that were sent out with the help of the function symbol if _then _else _. We illustrate this in the following example.

Example 2.2 The folded symbolic execution of one session DH protocol between an initiator $A$ and a responder $B$ has the following trace:

- $\phi_{0} \equiv G, g$

- $\phi_{1} \equiv \phi_{0}, t_{1}$

$$
\begin{aligned}
& \text { where } t_{1} \equiv \quad \text { if } \mathrm{EQ}\left(\operatorname{to}\left(f_{0}\left(\phi_{0}\right)\right), \mathrm{id}_{1}\right) \& \mathrm{EQ}\left(\operatorname{act}\left(f_{0}\left(\phi_{0}\right)\right), \text { new }\right) \\
& \text { then } g^{a_{1}} \\
& \text { else if } \mathrm{EQ}\left(\operatorname{to}\left(f_{0}\left(\phi_{0}\right)\right), i \mathrm{~d}_{1}\right) \\
& \text { then } g^{a_{1}} \\
& \text { else if } \mathrm{EQ}\left(\operatorname{to}\left(f_{0}\left(\phi_{0}\right)\right), \mathrm{id}_{2}\right) \& \mathrm{EQ}\left(\operatorname{act}\left(f_{0}\left(\phi_{0}\right)\right), \text { new }\right) \\
& \text { then } g^{a_{2}} \\
& \text { else } 0
\end{aligned}
$$

- etc,

where $G \equiv \pi_{1}\left(\operatorname{ggen}\left(n_{0}\right)\right)$ and $g \equiv \pi_{2}\left(\operatorname{ggen}\left(n_{0}\right)\right)$ and $a_{\alpha} \equiv \mathrm{r}\left(n_{m(\alpha)}\right)$ for $\alpha=1,2$ and some increasing function $m: \mathbb{N} \rightarrow \mathbb{N}$. Again remember, if the adversary calls for a new session $i d_{\alpha}$, then a new initiator session is started. If the adversary sends to sessions $i d_{\alpha}$ but does not call for a new session, then the agent starts a responder 
session, and assumes the incoming message is from the initiator. To obtain $\phi_{2}$, one proceeds the following way. First create a term that lists all conditions to reach all possible states after the first step:

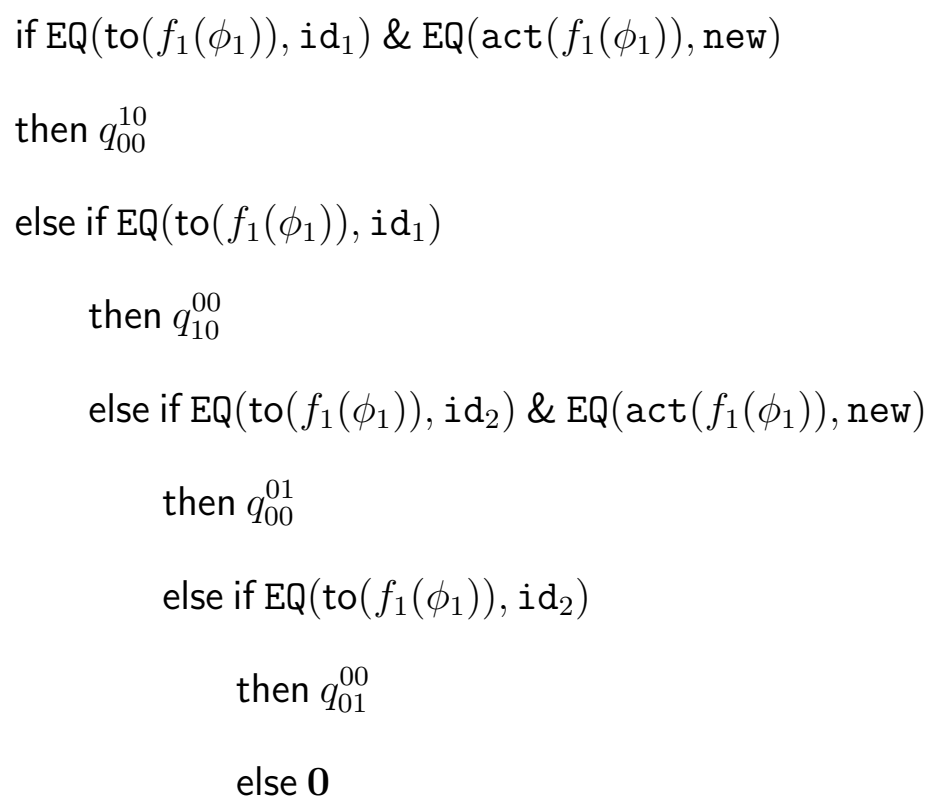

This way, the indistinguishability of two protocols $\Pi$ and $\Pi^{\prime}$ can be reduced to the indistinguishability of the lists of the sent messages.

\subsubsection{The soundness theorem}

Let fold $(\Pi)$ denote the folded execution of protocol $\Pi$, and let $\Phi($ fold $(\Pi))$ denote the sequence $\phi_{0}, \phi_{1}, \ldots$ of folded messages sent on the single symbolic trace. Then the following general soundness theorem was proved in [BCL14]:

Theorem 2.3 Let $\Pi, \Pi^{\prime}$ be two protocols. Let $\mathfrak{A}$ be any set of formulas (axioms). If $\mathfrak{A}$ and $\Phi($ fold $(\Pi)) \nsim \Phi\left(\right.$ fold $\left.\left(\Pi^{\prime}\right)\right)$ are inconsistent, then the protocols $\Pi$ and $\Pi^{\prime}$ are computationally indistinguishable in any computational model $\mathcal{M}^{c}$ for which $\mathcal{M}^{c} \models$ A. 


\subsubsection{Computationally Valid Axioms}

A core set of valid axioms of the CCSA have been given in [BC16] and are reproduced in the Table 2.1. The axioms use the notion of freshness that has been defined in [BCL14] and is reproduced below as follows: For any list of names $\vec{n}$, and a (possibly empty) list of terms $\vec{v}, \operatorname{fresh}(\vec{n} ; \vec{v})$ is the constraint that the names in $\vec{n}$ are pairwise distinct and that none of the names in $\vec{n}$ occur in $\vec{v}$.

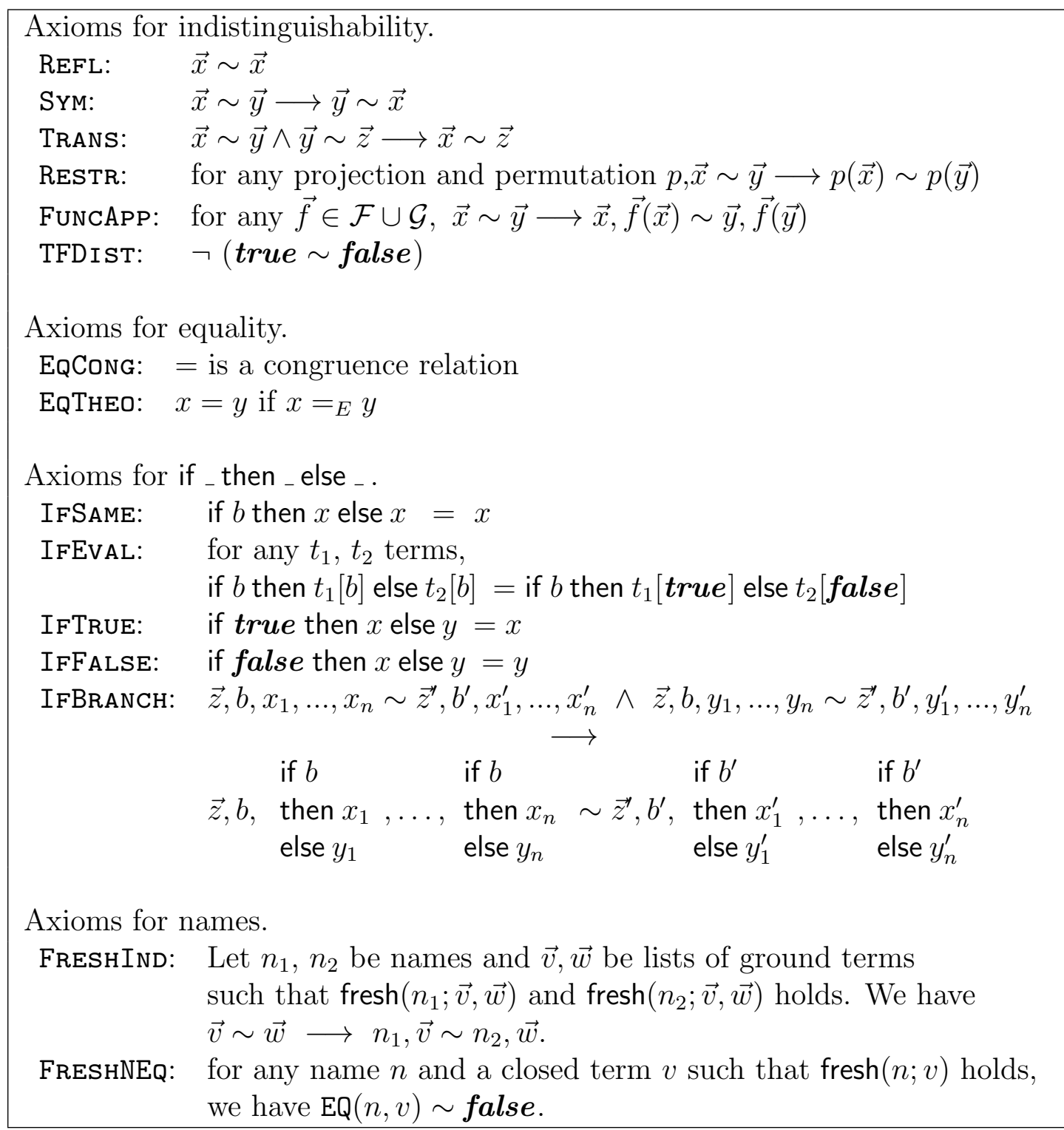

Table 2.1: Core Axioms

Essentially, these core axioms can be divided into four sets. The first set of 
axioms reflect the properties of the indistinguishability predicate $\sim$, namely, that it is an equivalence relation and is preserved under projection, permutation and function application. The second set of axioms says that the abbreviation $=$ is a congruence and preserves the equational theory $=_{E}$. The third set of axioms reflect the properties of the function symbol if_then_else_ such as if true then $x$ else $y=x$. Finally, we have a couple of axioms that reflect the truly random nature of names such as replacing any name $n_{1}$ by a fresh name $n_{2}$ yields indistinguishable sequence of terms. The interested reader is referred to $[\mathrm{BC} 16]$ for additional details, where several small examples of usage of the axioms are also given.

We recall some of the example proofs from [BCEO19] and list them in the following theorem.

Theorem 2.4 Assuming the core axioms the following theorems are proved in [BCEO19]:

1. IfIDEMP : if $b$ then (if $b$ then $x_{1}$ else $y_{1}$ ) else (if $b$ then $x_{2}$ else $y_{2}$ ) $=$ if $b$ then $x_{1}$ else $y_{2}$.

2. IfMorph : $f\left(z_{1}, \ldots\right.$, if $b$ then $x$ else $\left.y, \ldots, z_{n}\right)=\begin{array}{r}\text { if } b \text { then } f\left(z_{1}, \ldots, x, \ldots, z_{n}\right) \\ \text { else } f\left(z_{1}, \ldots, y, \ldots, z_{n}\right) \text {. }\end{array}$

3. IFTF : if $b$ then true else false $=b$.

4. IFIDEMP, IFMORPH, EqREFL, EQCONG $\vdash$

$$
\begin{gathered}
\text { if } b \text { then } t_{1}\left[\text { if } b \text { then } x_{1} \text { else } y_{1}\right] \\
\text { else } t_{2}\left[\text { if } b \text { then } x_{2} \text { else } y_{2}\right]
\end{gathered}=\begin{array}{r}
\text { if } b \text { then } t_{1}\left[x_{1}\right] \\
\quad \text { else } t_{2}\left[y_{2}\right] .
\end{array}
$$

5. For any constant $f \in \mathcal{F} \cup \mathcal{G}$, Trans, Restr, FuncApP, EqReFl $\vdash x \sim f \longrightarrow x=f$.

6. Trans, Restr, FunCApP, IfSAme, IfIdemp, IFMorph, IFTF, EqREFL, EqCong $\vdash$ if $\operatorname{EQ}\left(x_{1}, x_{2}\right)$ then $x_{1}$ else $y=$ if $\operatorname{EQ}\left(x_{1}, x_{2}\right)$ then $x_{2}$ else $y$.

7. IfIdemp, IfMorph, EqReFL, EQCong $\vdash$ if $b$ then $x_{1}$ else $y_{1}=$ if $b$ then $x_{2}$ else $y_{2}$ $\longrightarrow$ if $b$ then $t\left[x_{1}\right]$ else $t^{\prime}\left[y_{1}\right]=$ if $b$ then $t\left[x_{2}\right]$ else $t^{\prime}\left[y_{2}\right]$ 
8. $\mathrm{EQBRANCH} \equiv$ if $\mathrm{EQ}\left(x_{1}, x_{2}\right)$ then $t\left[x_{1}\right]$ else $t^{\prime}=$ if $\mathrm{EQ}\left(x_{1}, x_{2}\right)$ then $t\left[x_{2}\right]$ else $t^{\prime}$.

9. $\mathrm{EQ}($ true, false $)=$ false.

10. $\mathrm{EQ}(x, y)=\mathrm{EQ}(y, x)$.

11. By the invertibility of pairing, we can also show that for two distinct names $n_{1}$ and $n_{2}, \mathrm{EQ}\left(n_{1},\left\langle n_{1}, n_{2}\right\rangle\right)=$ false.

12. It is easy to see from the definition of not, that

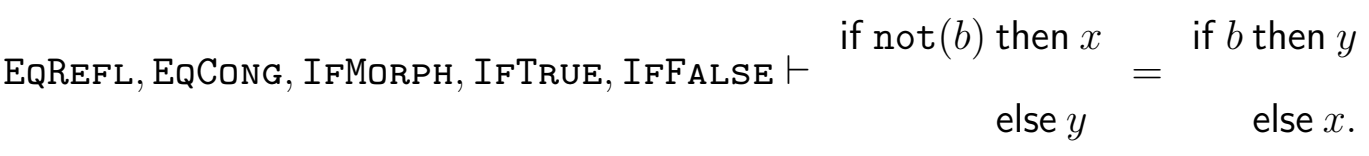




\section{Chapter 3}

\section{FOO Protocol and its Analysis using CCSA}

In this chapter, we analyze the FOO electronic voting protocol in the provable security model using the BC technique. The protocol uses commitments, blind signatures, and anonymous channels to achieve vote privacy. We model anonymous communication using a mix-net server who is responsible for receiving encrypted messages from the attacker and decrypts them and reveal in the decrypted messages in the lexicographic order. We discover about four new attacks on vote privacy, a computational attack due to unequal vote lengths, a computational attack due to uneven blind signature lengths, a Dolev-Yao style replay attack, and a man-in-the-middle attack.

We briefly recall the computational definitions of the primitives of the FOO protocol from the literature and axiomatize their assumptions such as computational hiding and binding of the commitments, blindness property of the blind digital signatures, and the shuffling capability of the mixer in the $\mathrm{BC}$ technique. We prove the validity of these assumptions in the computational setting of the CCSA framework. We also propose other hypotheses including all the vote values, candidates, blind signatures are on equal length messages, are of the same length and argue that these 
are necessary to avoid the attacks mentioned above. We give a game-based definition of vote privacy of the FOO protocol and present a labelled transition system for authentication, voting, and opening, of the protocol. Note that most of the content of this chapter is from the publication [BCE18].

\subsection{Introduction}

The FOO protocol was introduced by Fujioka, Okamoto, and Ohta in [FOO93]. It was one of the first protocols for large-scale secure electronic voting. The design was supposed to achieve fairness, eligibility, vote privacy and individual verifiability. Since it's publication, it has been the subject of several attempts to formalize and verify its security properties. The focus of this work is the formal analysis of vote privacy of FOO, namely, the property that the votes of honest voters cannot be linked to the voters. There are more modern protocols nowadays such as [Adi08, RRI16]. We chose FOO because of its abstract formulation, which make it convenient for symbolic analysis and its numerous phases which makes it prone to attacks.

As far as we are aware of, the FOO protocol was the first electronic voting protocol that was formally specified and analyzed [KR05]. In the seminal work of Kremer and Ryan [KR05], both the formalization and analysis of the FOO protocol was carried out in the Dolev-Yao (DY) attacker model using the applied pi-framework [ABF17]. The vote privacy property was the most intricate property analyzed in [KR05], and Kremer and Ryan were only able to prove vote privacy by hand. Subsequent development in DY verification allowed the proof to carried out automatically [DRS08, CCCK16]. As the DY model makes the assumption of perfect cryptography, the question of whether the proof carries over in the provable security model (computational model) remained unanswered.

In this Chapter, we formally verify vote privacy in the provable security framework, 
using symbolic verification techniques. In particular, we use the computationally complete symbolic attacker technique for indistinguishability properties introduced by Bana and Comon in [BCL14] (a.k.a. CCSA framework). As far as we know, this is the first formal analysis of FOO protocol in the provable security model. Besides proving vote privacy for FOO, our other aim is to further develop the library of axioms of the CCSA framework and to demonstrate its effectiveness in attack detection and verification.

CCSA technique was first introduced by Bana and Comon in [BCL12] for reachability properties and then for indistinguishability properties in [BCL14]. Since then it has been used to find new attacks to the Needham-Schroeder-Lowe protocol [BAS12a, BAS12b]; to treat algebraic operations notoriously difficult to reason about in the DY model, such as exponentiation along with it the decisional Diffie-Hellmann property and versions of the Diffie-Hellman key-exchange protocol [BCEO19]; to verify unlinkability of RFID protocols [CK17]; and to analyze key wrapping API's [SS16]. Automated tool is not yet available for the indistinguishability technique, but work is in progress. In the meantime, we continue developing the library of axioms, and verifying relatively simple protocols by hand.

FOO protocol assumes two election authorities: administrator and collector. The protocol proceeds in three phases: In the first phase, voters prepare their ballots in the form of a trapdoor commitment of their votes and obtain a blind signature on the ballots from the administrator indicating that they are eligible to vote. In the second, voters send their ballots to the collector using an anonymous channel who publishes them on a public bulletin board (BB). In the final phase, each voter verifies the presence of their ballots on the BB and then sends their trapdoor key along with the entry number of their commitment on bulletin board to the collector, again via an anonymous channel. The trapdoor keys are then also added to the bulletin board next to the commitments. After the votes finish, votes are tallied from BB. The creators 
of the FOO protocol did not specify how the anonymous channels are implemented. In our modeling, we model anonymous channel as a mix-net server [Cha81] which, upon receiving a list of encrypted messages outputs their plaintexts in lexicographic order after decrypting them.

FOO protocol is designed to provide vote privacy even when the administrator and collector are corrupt and our analysis of vote privacy assumes this to be the case. As in [KR05, BT94, Ben87], vote privacy is modeled as indistinguishability of two protocol executions: in one, honest voter $A$ votes for candidate $v_{1}$ and honest voter $B$ votes for candidate $v_{2}$ and in the other, honest voter $A$ votes for candidate $v_{2}$ and honest voter $B$ votes for candidate $v_{1}$. Observe that, as stated above, FOO protocol does not satisfy vote privacy as the attacker may choose to forward only Alice's trapdoor key in the final phase of the protocol to the mix-net server. We argue that privacy of votes can never be guaranteed for any voting protocol if the attacker allows only one participant to complete the protocol. Hence, our formalization is carefully crafted to avoid these cases.

Our analysis revealed new attacks on the FOO protocol (See Section 3.4.2). The first attack occurs because of an inadequacy of blindness property of blind signatures. Intuitively, blindness [JLO97] means that a dishonest signer who engages in two sessions (parallel or sequential) with an honest user on messages $m_{0}$ and $m_{1}$ cannot detect which session is for $m_{0}$ and which session is for $m_{1}$ if the user successfully outputs signatures in both sessions. The blindness property, however, does allow the possibility that the attacker can distinguish between the sessions if the user is successful in only one session. In order to prevent this attack, we have to assume that the identities of the candidates are of equal length. This attack does not depend on the implementation of the anonymous channel. The second attack exploits the fact that encryption scheme used by the mix-net server may be length-revealing and hence the length of the encrypted messages to the mix-net server may reveal their senders. 
In order to prevent this, we have to assume that the signatures obtained on equal length messages by executing the blind signature interactive protocol with the same signer must be of equal length. The above two attacks lie outside the DY model and hence were not detected in previous works on formal analysis of FOO protocol. A third attack is a DY style replay attack in which messages from the Voting phase can be replayed in the Opening phase. This attack can be prevented by introducing phase numbers in the FOO protocol. Finally, we point out a man-in-the-middle attack in which a dishonest voter intercepts one of the two ballots of the honest voters and sends some garbage to the mixer instead. Then in the opening phase, the attacker creates a bulletin board which contains all the ballots of the honest voters. Unaware to this situation, the honest voters follow the protocol and reveals their commitment keys in the opening phase. This attack can be prevented by generating and including a nonce in the ballots of honest voters in the voting phase.

With these additional assumptions, we establish vote privacy of the FOO protocol for one session with two honest voters and one dishonest voter. The proof carries over to $n$ dishonest voters for any fixed $n$. The proof of vote privacy rests on the blindness property of the blind signature, the computational hiding property of the trapdoor commitments and of IND-CCA2 assumption on the encryptionused in the anonymizing mechanism. The proof of vote privacy in the DY model, in contrast, relies only on the blindness property. ${ }^{1}$ The commitment hiding property of trapdoor commitments does not play a role in establishing vote privacy in the DY analysis.

\subsection{FOO Voting Protocol}

We briefly recall the electronic voting protocol FOO introduced by Fujioka, Okamoto, and Ohta in [FOO93]. We assume that the reader is familiar with the cryptographic

\footnotetext{
${ }^{1}$ As evidence, we ran AKiSs [CCCK16] on a two-phase variant of the FOO protocol without commitments. The variant satisfies the vote privacy property in the DY model.
} 


\section{Authentication :}

$1: V_{i}$ : computes $c_{i}:=\xi\left(v_{i}, k_{i}\right)$, a commitment on her vote $v_{i}$ with trapdoor $k_{i}, b_{i}:=\chi\left(c_{i}, r_{i}\right)$,

blinding the ballot $c_{i}$ with blinding key $r_{i}$, and $s_{i}:=\operatorname{sign}_{i}\left(b_{i}\right)$, a signature on $b_{i}$

$2: V_{i} \rightarrow \mathrm{A}:\left\langle I D_{i}, b_{i}, s_{i}\right\rangle$

$3:$ A : verifies the signature $s_{i}$ and eligibility (not yet applied for his signature) of $V_{i}$

$4: \mathrm{A} \rightarrow V_{i}: \operatorname{bsign}_{\mathrm{A}}\left(b_{i}\right)$, (blind) signature of $\mathrm{A}$ on the blinded ballot $b_{i}$

\section{Voting :}

$5: V_{i}:$ checks if the received message is A's blind signature on $b_{i}$; if the check

passes, $V_{i}$ unblinds the signature and it is denoted by $\sigma_{\mathrm{A}}\left(c_{i}\right)$

$6: V_{i} \rightarrow \mathrm{C}:\left\langle c_{i}, \sigma_{\mathrm{A}}\left(c_{i}\right)\right\rangle$

$7: \mathrm{C}$ : verifies the signature, and adds the received message to Bulletin Board (BB) with label $\ell_{i}$

8: C : after a certain time, $\mathrm{C}$ publishes the BB

\section{Opening :}

$9: V_{i}:$ finds her ballot on the $\mathrm{BB}$ and records its label $\ell_{i}$

$10: V_{i} \rightarrow \mathrm{C}:\left\langle\ell_{i}, k_{i}\right\rangle$

11: C : opens the commitments at $\ell_{i}$ using the key $k_{i}$, and appends $\left\langle k_{i}, v_{i}\right\rangle$ to

$\left\langle c_{i}, \sigma_{\mathrm{A}}\left(c_{i}\right)\right\rangle$ in the $\mathrm{BB}$, and after a certain time, publishes the new $\mathrm{BB}$

$12: \mathrm{C}:$ checks the validity of the votes, counts them and publishes the result

Figure 3.1: FOO Voting Protocol

primitives of public key encryption, trapdoor commitment schemes and digital signatures schemes. The FOO protocol also uses blind signature schemes. Informally, a blind signature scheme is an interactive protocol that allows a party $\mathcal{U}$ to obtain the signature of a party $\mathcal{S}$ on a message that is obfuscated by $\mathcal{U}$ until $\mathcal{S}$ completes the protocol and $\mathcal{U}$ publishes the signed message. We assume as in [CRS83, ANN06] that the interactive protocol consists of three phases: blinding by $\mathcal{U}$, (blind) signing by $\mathcal{S}$ and unblinding by $\mathcal{U}$. The primitives are described in detail in Section 3.3.

The FOO protocol has three roles: voters $\left(V_{i}\right)$, administrator $(\mathrm{A})$, and collector (C). It assumes the existence of anonymous channels. Following Kremer et al [KR05], we group the (original) protocol in three phases, Authentication, Voting, and Opening, as described in Figure 3.1. In the Figure, $\rightarrow$ means send, $\rightarrow$ means send via anonymous channel.

Authentication Phase: In the Authentication phase, each voter $V_{i}$ creates her 
ballot, $c_{i}$, by applying a trapdoor commitment scheme on her vote $v_{i}$. She blinds the ballot as stipulated by the blind signature technique. The result of the blinding is henceforth denoted by $b_{i}$. The blinded ballot, $b_{i}$ is then signed by $V_{i}$ using a standard signature scheme and the result is henceforth denoted by $s_{i}$. Then, $V_{i}$ creates the triple $\left\langle I D_{i}, b_{i}, s_{i}\right\rangle$ where $I D_{i}$ is her identity and sends the triple to the administrator $A$. The administrator receives the message from the voter and verifies that $I D_{i}$ is eligible to vote and has not previously applied for $A$ 's signature. If the checks succeed, the administrator acknowledges that by signing the blinded message as stipulated by the blind signature technique. Otherwise he aborts the signing process for the applicant $V_{i}$. At the end of this phase, the administrator publishes the list $\left\langle I D_{i}, b_{i}, s_{i}\right\rangle$ consisting of all votes for whom he (blindly) signed the ballots.

Voting Phase: In the Voting phase, voter $V_{i}$ checks if the message sent by $A$ is $A$ 's blind signature on $b_{i}$. If yes, it unblinds the message and obtains $A$ 's signature on the ballot $c_{i}$. The unblinded message $\sigma_{A}\left(c_{i}\right)$ is sent to the collector through an anonymous channel (dashed arrow in Figure 3.1). The collector $C$ verifies the administrator's signature on $c_{i}$, adds the ballot $c_{i}$ and the signed ballot $\sigma_{A}\left(c_{i}\right)$ to the Bulletin Board (BB) and labels it with a position $l_{i}$. After a certain time, the collector publishes BB. Opening Phase: Each voter $V_{i}$ checks whether her ballot appears on BB. If the checks succeed, the voter sends the respective label and commitment key to $C$ through the anonymous channel. The collector opens the ballot and checks if the vote is valid. If it is valid, he appends the commitment key at the respective label. After a certain time, $C$ publishes the new BB, counts the votes and announces the result.

\subsubsection{Anonymous Channel}

The creators of FOO did not specify how to implement anonymous channels. We now clarify our modeling of the anonymous channels. We model the anonymous communication using a mix-net server which, upon receiving a list of messages encrypted 
with its public key, checks if they are all different, decrypts each message in the list, outputs them in lexicographic order. Henceforth, we refer to mix-net server as the mixer. In particular, in the Voting phase (resp. Opening phase), the voter encrypts $\left\langle c_{i}, \sigma_{\mathrm{A}}\left(c_{i}\right)\right\rangle\left(\operatorname{resp} .\left\langle l_{i}, k_{i}\right\rangle\right)$ with the mixer $M$ 's public key $p k_{M}$. The mixer waits until a certain time during which receives the messages from the voters, checks if all messages received until that time are distinct. If the check succeeds, the mixer decrypts the messages, shuffles the decrypted messages into lexicographic order and outputs them.

\subsection{Computational Modeling of the FOO protocol}

As usual, we assume that all agents in the protocol execution, the voters, the administrator, the collector are interactive probabilistic polynomial-time Turing machines. Furthermore, the network is controlled by an attacker, which is also an interactive PPT machine. Each message goes through the attacker, except those that are "published", in which case the message is written directly on each participant's work tape synchronously. The Bulletin Board BB is simply a list of tuples. We assume that the id's $I D_{i}$ are the same as the voters' public keys used for verifying their digital signatures.

We assume that encryption used in the protocol is indistinguishable against adaptive chosen ciphertext attack, i.e., satisfies the IND-CCA2 property. The interested reader is referred to [Sma16]. Digital signatures are used in the protocol to ensure that only eligible voters vote and do not play a part in establishing vote privacy. We now present the computational modeling of the commitment and blind digital signature schemes, and their security properties we assume for the verification. 


\subsubsection{Commitments}

A commitment scheme allows one party, say $V$, to commit to a value obfuscating the value it committed to until it is revealed by $V$ with the help of the commitment key. In general, a commitment scheme satisfies two properties: binding and hiding. The former property states that the party $V$ cannot change the message once $V$ committed to it while the latter property says that it should be computationally infeasible for any other party to retrieve the message from the commitment unless the $V$ reveals the message and the key.

Formally, a commitment scheme [Sma16] $\mathcal{C}$ is a pair of algorithms (KGen, com) such that KGen is a PPT algorithm with input $1^{n}$, while com is a PT algorithm such that there is a polynomial poly $(\cdot)$ such that for each security parameter $n$ and bitstring $m$ of length at most poly $(n), \operatorname{com}(m, \mathbf{k})$ computes the commitment on message $m$ for $\mathrm{k} \leftarrow \mathrm{KGen}\left(1^{n}\right)$. We assume that the commitment schemes are length regular. That is if $m_{0}$ and $m_{1}$ are messages of equal length then the commitments of $m_{0}$ and $m_{1}$ are of equal length [DPP97, Nao91].

The computational hiding property is formalized as a game between a (PPT) attacker and a (honest) challenger. Initially, the attacker generates two messages $m_{0}$ and $m_{1}$ of equal length, which it gives to the challenger. The challenger creates commitments, $c o m_{0}$ and $c o m_{1}$ for $m_{0}$ and $m_{1}$ with different (secret) commitment keys and sets a secret bit $b$ with uniform probability. The challenger then gives the ordered pair $\left(\mathrm{com}_{b}, \mathrm{com}_{1-b}\right)$ to the attacker who wins if it correctly guesses the value of $b$. The commitment scheme is said to satisfy the commitment hiding property [Sma16] if the winning probability of the attacker cannot be non-negligibly different from $\frac{1}{2}$. Formally,

Definition 3.1 (Computationally Hiding [Sma16]) A commitment scheme $\mathcal{C}=($ KGen, com $)$ is computationally hiding [Sma16] if for any PPT algorithm $\mathcal{A}$ (working in modes find and guess) the probability that the following experiment 
$\operatorname{Hiding}_{\text {com }}^{\mathcal{A}}(n)$ evaluates to 1 is negligibly different from $1 / 2$.

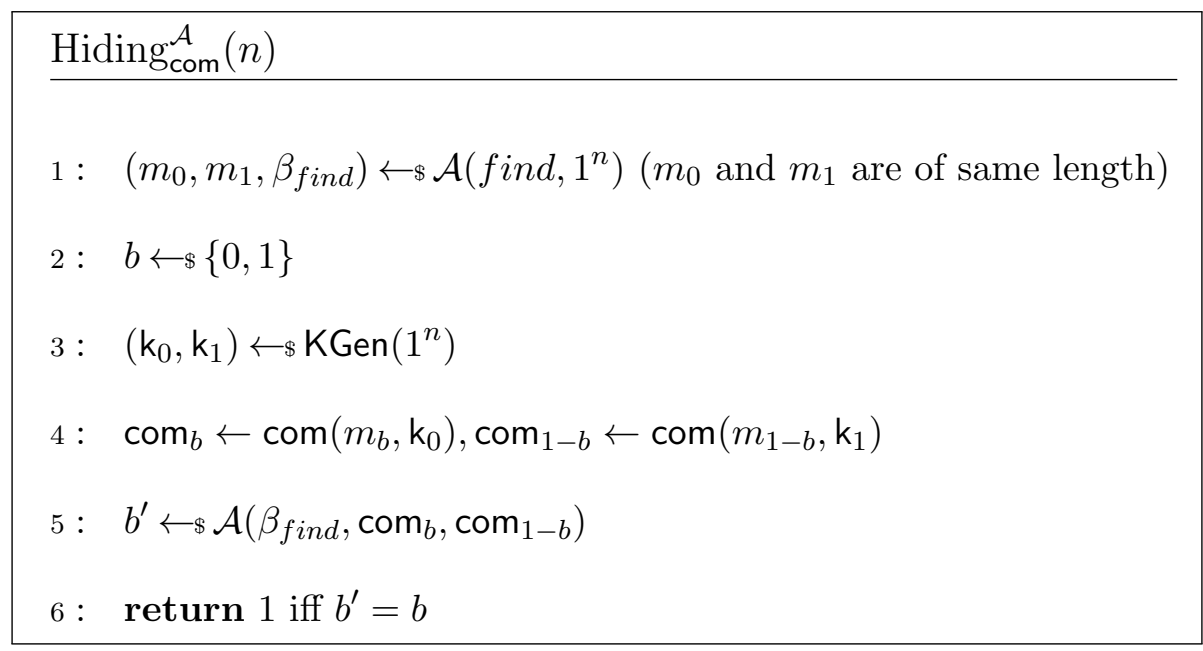

Figure 3.2: The hiding experiment $\operatorname{Hiding}_{\text {com }}^{\mathcal{A}}(n)$ of the commitments

The computational binding property is also formalized as a game between an attacker and a challenger in which the attacker has to produce $c, m, \mathbf{k}, m^{\prime}$, and $\mathbf{k}^{\prime}$ with $m \neq m^{\prime}$ and $c=\operatorname{com}(m, \mathbf{k})$ such that $c=\operatorname{com}\left(m^{\prime}, k e y^{\prime}\right)$. The commitment scheme is said to satisfy the computational binding property [Sma16] if the winning probability of the attacker is negligible. Formally,

Definition 3.2 (Computationally Binding [Sma16]) A commitment scheme $\mathcal{C}=($ KGen, com $)$ is computationally binding [Sma16] if for any PPT algorithm $\mathcal{A}$ the probability that the following experiment $\operatorname{Binding}_{\text {com }}^{\mathcal{A}}(n)$ evaluates to 1 is negligible.

$\operatorname{Binding}_{\text {com }}^{\mathcal{A}}(n)$
$1: \quad\left(c, m, \mathrm{k}, m^{\prime}, \mathrm{k}^{\prime}\right) \leftarrow \Phi \mathcal{A}\left(1^{n}\right)\left(m^{\prime} \neq m\right.$ and $\left.c=\operatorname{com}(m, \mathrm{k})\right)$
$2: \quad \operatorname{return} 1$ iff $\operatorname{com}\left(m^{\prime}, \mathrm{k}^{\prime}\right)=c$

Figure 3.3: The binding experiment $\operatorname{Binding}_{\text {com }}^{\mathcal{A}}(n)$

\subsubsection{Blind Digital Signatures}

Blind signature schemes were introduced by Chaum [CRS83]. They are small interactive protocols between a user and a signer. Informally, a blind signature scheme is 
an interactive protocol that allows a party $\mathcal{U}$ to obtain the signature of a party $\mathcal{S}$ on a message that is obfuscated by $\mathcal{U}$ until $\mathcal{S}$ completes the protocol and $\mathcal{U}$ publishes the signed message.

Formally, a blind digital signature scheme [JLO97, ANN06] B is a tuple, (Gen, $\langle\mathcal{S}, \mathcal{U}\rangle$, verif), a PPT key generation algorithm Gen, an interactive algorithm $\langle\mathcal{S}, \mathcal{U}\rangle$ of the PPT singer $\mathcal{S}$ and the PPT user $\mathcal{U}$, and a PT verification algorithm verif, such that for each security parameter $n$ : $\operatorname{Gen}\left(1^{n}\right)$ outputs a public and private key pair $(p k, s k)$, the joint execution of signer $\mathcal{S}(p k, s k)$ and the user $\mathcal{U}(p k, m)$ on the message $m \in\{0,1\}^{n}$ produces an output $\sigma$ for the user, and the deterministic $\operatorname{algorithm} \operatorname{verif}(m, \sigma, p k)$ outputs a bit. The algorithm $\mathcal{U}(p k, m)$ may fail to produce an output; in which case $\mathcal{U}$ is said to abort and $\mathcal{U}(p k, m)$ said to be undefined. In that case, we write $\mathcal{U}(p k, m)=\perp$. A blind signature scheme is required to be complete, i.e., for all parameter $\eta$ the following holds: for all messages $m \in\{0,1\}^{n}$, if $(p k, s k) \leftarrow \operatorname{Gen}\left(1^{n}\right)$ then the joint execution of $\mathcal{S}(p k, s k)$ and $\mathcal{U}(p k, m)$ must be defined and the produced output $\sigma$ should be $\operatorname{such}$ that verif $(p k, m, \sigma)=1$.

Blind signatures should satisfy two security properties, blindness and unforgeability. Intuitively, unforgeability says a malicious user who engages in $\ell$ sessions with an honest user should not be able to produce $\ell+1$ valid message-signature pairs. In the FOO protocol, unforgeability is used to make sure only eligible voters vote. It does not play a role in vote privacy. For this reason, we omit the formalization of unforgeability. The interested reader is referred to [JLO97]. The blindness property [JLO97] on the other hand, is useful in establishing privacy of votes. Intuitively, blindness means that a dishonest signer cannot learn the contents of the message it signed. The blindness property is formalized as a game between a (PPT) attacker acting as the signer and a (honest) challenger. Initially, the attacker generates two messages $m_{0}$ and $m_{1}$, which it gives to the challenger. The challenger sets a secret bit $b$ with uniform probability. The challenger, acting as the user, then engages in 
two blind signature sessions with the attacker as the signer; the first is for obtaining signature on $m_{b}$ and the second is for obtaining signature on $m_{1-b}$. If the user successfully completes both sessions and obtains signature $\sigma_{b}, \sigma_{1-b}$ then it gives the ordered pair $\left(\sigma_{0}, \sigma_{1}\right)$ to the attacker. Otherwise, it gives the pair $(\perp, \perp)$ to the attacker. The attacker wins if it correctly guesses the value of $b$. Note that it is important that the challenger gives the pair $\left(\sigma_{0}, \sigma_{1}\right)$ and not $\left(\sigma_{b}, \sigma_{1-b}\right)$ (otherwise the attacker will win with probability 1). The blind signature scheme is said to satisfy the blindness property [JLO97] if the winning probability of the attacker cannot be non-negligibly different from $\frac{1}{2}$. Formally,

Definition 3.3 (Blindness [JLO97]) An interactive signature scheme $\mathrm{B}=\left(\mathrm{Gen},\langle\mathcal{S}, \mathcal{U}\rangle\right.$, verif) satisfies blindness [JLO97] if for any efficient algorithm $\mathcal{S}^{*}$ (working in modes find, issue and guess) the probability that the following experi$\operatorname{ment}_{\operatorname{Blind}}^{\mathrm{B}}(n)$ evaluates to 1 is negligibly close to $1 / 2$, where $\mathcal{S}^{*}\left\langle\cdot, \mathcal{U}\left(p k, m_{b}\right)\right\rangle^{1},\left\langle., \mathcal{U}\left(p k, m_{1-b}\right)\right\rangle^{1}$ represents that the signer $\mathcal{S}^{*}$ can invoke arbitrary ordered executions with $\mathcal{U}\left(p k, m_{b}\right)$ and $\mathcal{U}\left(p k, m_{1-b}\right)$ once:

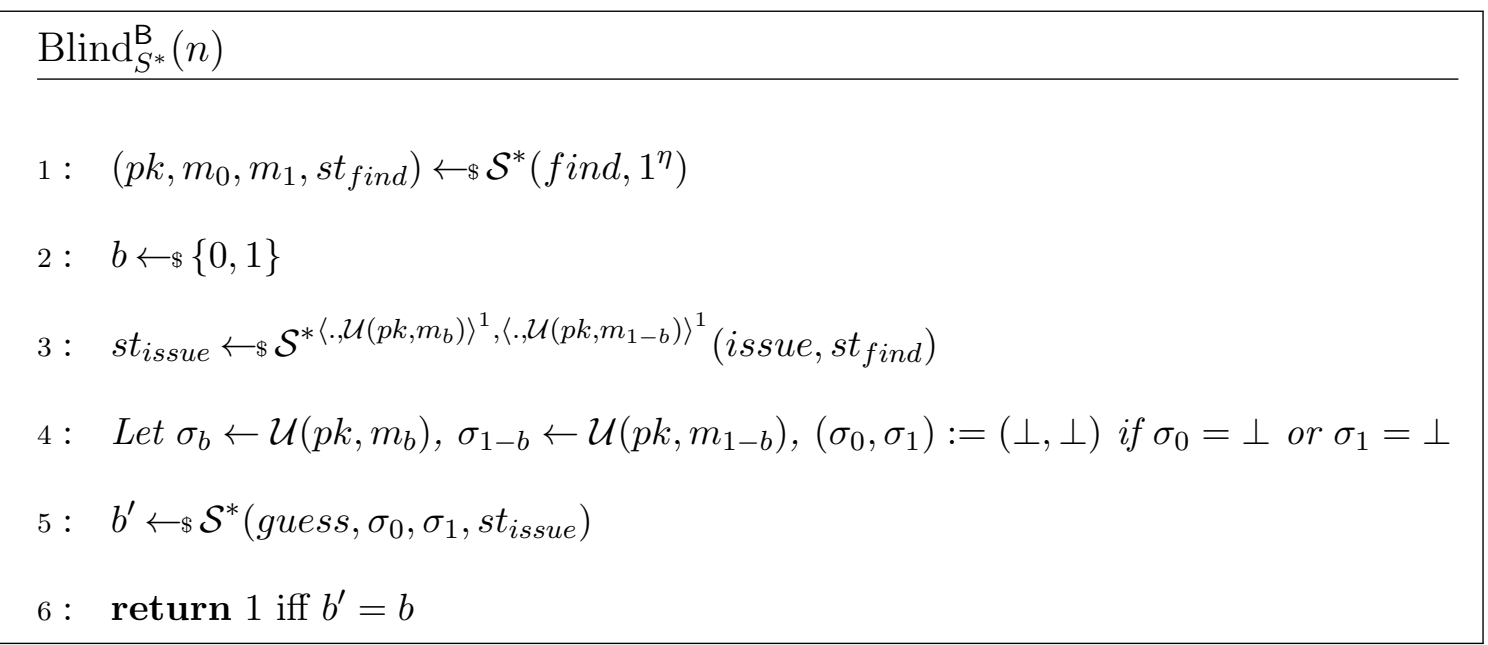

Figure 3.4: The blind signing experiment $\operatorname{Blind}_{S^{*}}^{\mathrm{B}}(n)$

We model the blind signatures using an interactive protocol between the user and the singer in three steps: blinding, blind signing, and unblinding and involving four 
algorithms, blind, bsign, accept, and unblind [CRS83, ANN06]. Before the protocol commences, the signer generates a public key and secret-key pair $(p k, s k)$ and publishes $p k$.

- Blinding: User computes $b:=b l i n d\left(m, p k, r_{b}\right)$ for a message $m$, using the signer's public key $p k$, and a random seed $r_{b}$, and sends $b$ to the signer.

- Blind signing: The signer computes $\rho:=b \operatorname{sign}\left(b, s k, r_{s}\right)$ using his own secret key $s k$ and a random seed $r_{s}$, and sends it to the user.

- Unblinding: User checks validity of $\rho$ by running accept $\left(m, p k, r_{b}, \rho\right)$. If it outputs false, the user quits the protocol without any output. If it outputs true, the user computes $\sigma=\operatorname{unblind}\left(m, p k, r_{b}, \rho\right)$.

\subsubsection{Digital Signatures}

In order to continue to demonstrate the usability of our technique, we also consider authentication that signatures can deliver. Digital signatures allow one party, say S (called signer), to sign a message in such a way that any other party who has the public key $p k$ of $\mathrm{S}$ can verify If the message is actually originated from S. In other words, the other party can detect if the message has been modified in some way. We now recall formal definition of a signature scheme from [KL07] below.

Definition 3.4 (Signature Scheme [KL07]) A signature scheme is a triple of algorithms $\left(\mathrm{KGen}_{\mathrm{s}}\right.$, Sign, Ver) such that:

- $\mathrm{KGen}_{\mathrm{s}}$ is a PPT key generation algorithm that, on a given security parameter $1^{n}$, outputs a public-secret key pair $(p k, s k)$.

- The signing algorithm Sign is a PPT algorithm that takes secret key $s k$, message $m \in\{0,1\}^{n}$, and a random seed $r$ as input and outputs a signature $\sigma \leftarrow \operatorname{Sign}(s k, m, r)$. 
- Ver is a deterministic algorithm that takes public key $p k$, message $m$, and signature $\sigma$ and outputs either 1 (valid) or 0 which means that the signature is invalid on $m$.

It is assumed that, for every $n$, every public-secret key pair $(p k, s k) \leftarrow \operatorname{KGen}_{\mathrm{s}}\left(1^{n}\right)$, and for every $m \in\{0,1\}^{n}$ with a random seed $r$, we have that $\operatorname{Ver}(p k, m, \operatorname{Sign}(s k, m, r))=$ 1.

In general, a signature scheme is existentially unforgeable under an adaptive chosenmessage attack (UF-CMA) which sates that it is infeasible for an adversary to produce a message $m$, which has not been signed by the signer S previously, along with a valid signature $\sigma$ on $m$ even if the adversary is allowed to obtain to signatures on many other messages of its choice. We reproduce the formal definition of UF-CMA [KL07] below.

Definition 3.5 (UF-CMA) A signature scheme $\Pi=\left(\mathrm{KGen}_{\mathrm{s}}\right.$, Sign, Ver $)$ is said satisfy the property of UF-CMA if for each PPT adversary $\mathcal{A}$, the probability that the following game Signforge ${ }_{\mathcal{A}, \Pi}^{\mathrm{cma}}(n)$ evaluates to 1 is negligible in $n$, i.e, $\operatorname{negl}(n)$. In $\operatorname{Signforge}_{\mathcal{A}, \Pi}^{\mathrm{cma}}(n), \mathcal{A}^{O_{\mathrm{Sign}(s k, .)}^{k}}\left(m_{1}, \ldots, m_{k}\right)$ represents that the adversary $\mathcal{A}$ can invoke at most $k$ queries the signing Oracle $O_{\operatorname{sign}(s k, .)}$ on messages $m_{1}$ and so on $m_{k}$ respectively.

$\begin{array}{ll} & \text { Signforge }_{\mathcal{A}, \Pi}^{\mathrm{cma}}(n) \\ 1: & (p k, s k) \leftarrow{ }_{\$} \operatorname{KGen}_{\mathrm{s}}\left(1^{n}\right) \\ 2: & \left(m_{1}, \ldots, m_{k}\right) \leftarrow \$ \mathcal{A}\left(1^{n}, p k\right) \\ 3: & \left(m_{1}, \sigma_{1}\right), \ldots,\left(m_{k}, \sigma_{k}\right),(m, \sigma) \leftarrow \$ \mathcal{A}^{O_{\operatorname{Sign}(s k, .)}^{k}}\left(m_{1}, \ldots, m_{k}\right) \\ 4: & \text { return } 1 \text { iff } \operatorname{Ver}(p k, m, \sigma)=1 \text { and } m \neq m_{i} \text { for } i=1, \ldots, k\end{array}$

Figure 3.5: The signature experiment Signforge ${ }_{\mathcal{A}, \Pi}^{\mathrm{cma}}(n)$ 


\subsubsection{Encryptions}

Encryptions allow one party to translate a message, often known as plaintext or clear text, into unreadable form, called ciphertext, such that only legitimate parties can translate back to the plaintext. The process of encoding from plaintext to ciphertext is called encryption while decoding back to plaintext from ciphertext is known as decryption. Broadly, encryptions are categorized into two: private-key and publickey encryptions. In the former, the encryption and the decryptions are performed using the same secret key $s k$ and is also known as symmetric key encryption. On the other hand, in the latter, the encryption is done using the public key $p k$ while decryption uses the respective secret key $s k$. These keys $p k$ and $s k$ may be related in some way. This technique also known as asymmetric encryption. Formally,

Definition 3.6 (Public-key encryption scheme) A public-key encryption scheme is a triple of algorithms $(\mathcal{K}, \mathcal{E}, \mathcal{D})$ such that:

- The PPT algorithm $\mathcal{K}$ takes $1^{n}$ as an input and outputs a public-secret key pair $(p k, s k)$.

- The PPT algorithm $\mathcal{E}$ takes as input a public key $p k$, for a polynomial $p($.$) and$ a message $m \in\{0,1\}^{p(n)}$, and outputs a ciphertext $c \leftarrow \mathcal{E}(m, p k)$.

- The deterministic algorithm $\mathcal{D}$ takes a ciphertext $c$ and a secret key $s k$ as input and outputs a plaintext $m$ or a special symbol $\perp$ indicating failure. We write it as $m:=\mathcal{D}(m, s k)$.

It is assumed that for every security parameter $n$, every $(p k, s k) \leftarrow \mathcal{K}\left(1^{n}\right)$, for a polynomial $p($.$) and every message m \in\{0,1\}^{p(n)}$, the following holds:

$\mathcal{D}(\mathcal{E}(m, p k), s k)=m$

There are numerous security notions available for encryptions (see e.g. [BDPR98]) such as IND-CPA, IND-CCA1, IND-CCA2 and so forth. However, we limit our focus 
to the notion of Indinstinguibility under adaptive Chosen Ciphertext Attack (INDCCA2) which is the only assumption about encryptions we use in our proofs and it is stronger than IND-CPA and IND-CCA1.

Definition 3.7 (IND-CCA2) A public-key encryption scheme $\Pi=(\mathcal{K}, \mathcal{E}, \mathcal{D})$ has indistinguishable encryptions under adaptive chosen-ciphertext attack if for each PPT adversary $\mathcal{A}$ the probability that the the following game $\operatorname{PubK}_{\mathcal{A}, \Pi}^{\mathrm{cca} 2}(n)$ evaluates to 1 is negligibly close to $\frac{1}{2}$.

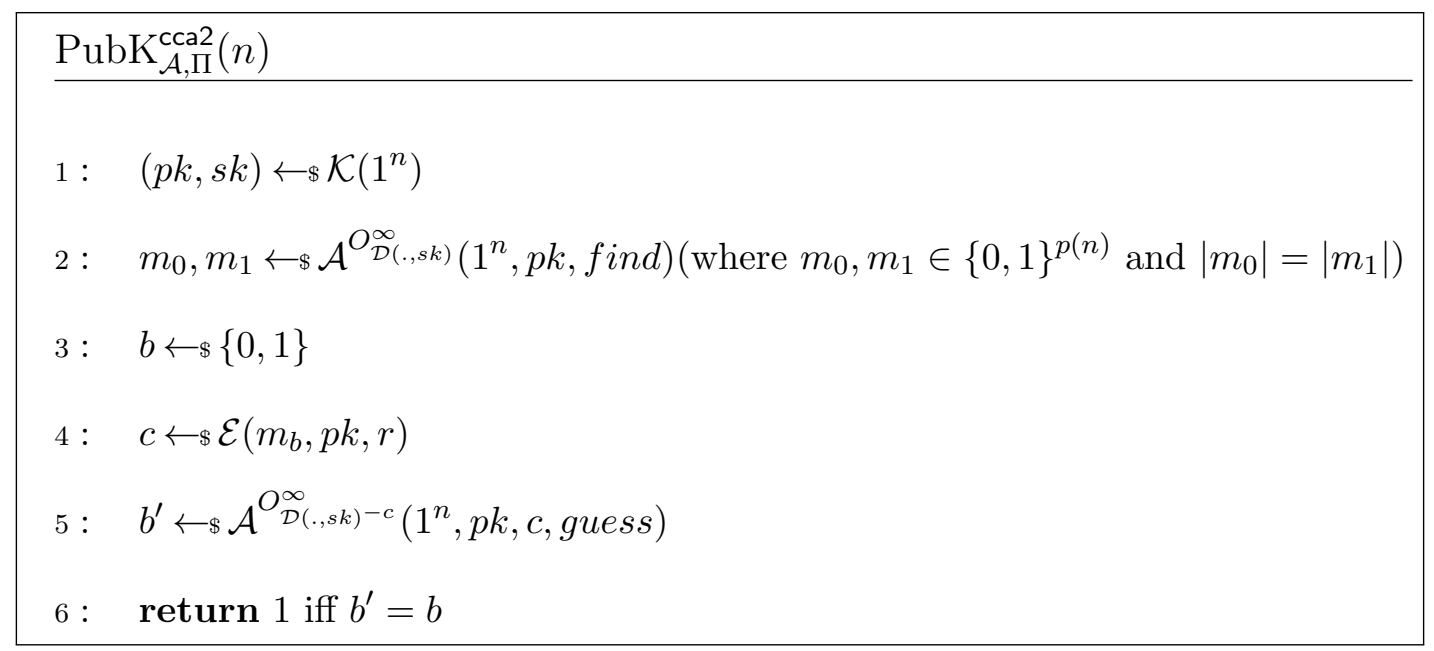

Figure 3.6: The adaptive CCA experiment $\operatorname{PubK}_{\mathcal{A}, \Pi}^{\mathrm{cca} 2}(n)$

Here $\mathcal{A}^{O_{\mathcal{D}(., s k)}^{\infty}}$ represents that the adversary $\mathcal{A}$ is allowed to access the Decryption Oracle $\mathcal{D}(., s k)$ any number of times on the messages of his choice. $\mathcal{A}^{O_{\mathcal{D}(., s k)^{-c}}^{\infty}}$ represents that the adversary can query the Decryption Oracle any number of times on the messages of his choice except on the challenged ciphertext $c$.

\subsection{Vote privacy for the FOO protocol}

We describe the targeted security notion of the voting, namely, that votes of honest voters remain secret. The fundamental idea of vote privacy in the literature is that permutation of votes cast by honest voters cannot be detected by the attacker. There 
are numerous attempts to formalize this idea such as $\left[\mathrm{SB} 13, \mathrm{BCG}^{+} 15\right]$. A major distinction between the protocols considered in $\left[\mathrm{KR} 05, \mathrm{BCG}^{+} 15\right]$ and $\mathrm{FOO}$ protocol is that unlike the single-phase protocols considered therein, the Bulletin Board (BB) is built incrementally in FOO in different phases. Hence, we have to adapt the definition of privacy of votes to FOO protocol. We describe our formalization below. We consider only one session.

\subsubsection{Formalization of Vote Privacy}

We assume that the attacker controls the network and we allow the administrator and the collector to be corrupt. We require that the flipping of votes of two honest voters should result in computationally indistinguishable runs to any PPT attacker even if he controls all other voters. Under these assumptions however, there is an obvious unavoidable attack. Suppose $A$ votes $v_{0}$ and $B$ votes $v_{1}$, while in the flipped voting scenario, $A$ votes $v_{1}$ and $B$ votes $v_{0}$. If the attacker allows the ballots of both $A$ and $B$ to be posted in the Voting phase, but subsequently blocks $B$ 's message in the opening phase, then the two situations will be distinguishable. This is because in the first scenario only $v_{0}$ vote will appear on the BB, while in the second, only $v_{1}$. Therefore, our formalization requires that the scenario with the original votes and with the flipped votes be indistinguishable only when either both voters' votes appear on the $\mathrm{BB}$ or neither does.

Accordingly, we require the following two games to be computationally indistinguishable to a PPT attacker. For bit $b$, let $\Pi_{b}$ be the following game between the Attacker and the Challenger simulating two honest voters of the FOO protocol and the mixer but aborting when only one of the commitment keys reach the mixer. The attacker simulates the corrupt administrator, the corrupt collector, and at the end has to guess the value of $b$ :

1. Challenger publishes public keys of $A, B$, and the mixer, and a list of candidates. 
2. Attacker publishes possibly dishonestly generated public keys of other voters, the administrator, and the collector. It then gives Challenger two valid votes $v_{0}$ and $v_{1}$.

3. Challenger creates the ballots of $v_{b}$ for $A$ and $v_{1-b}$ for $B$.

4. Attacker calls one of $A$ or $B$ to authorize vote with administrator.

5. Challenger prepares the corresponding blinded ballot. It sends the corresponding identity, the blinded ballot, and the corresponding signature to the attacker.

6. Attacker creates the possibly fake authorization, sends it back to the voter, and calls for the other voter to carry out the authorization.

7. Challenger prepares the appropriate blinded ballot and sends it to the attacker.

8. Attacker creates and sends again the authorization now for the other voter, and asks either $A$ or $B$ to proceed with sending the ballot.

9. If the corresponding blind signature was accepted, Challenger unblinds it, generates a nonce and sends the signed ballot along with the nonce and the phase number encrypted with the mixer's public key. Otherwise skips.

10. Attacker makes some computations and sends a message back (which is possibly the unchanged ciphertext sent in the previous step).

11. If the other of $A$ or $B$ 's blind signature was accepted, Challenger now unblinds that, generates a nonce and sends the signed ballot along with the nonce and the phase number encrypted with the mixer's public key. Otherwise skips.

12. Attacker makes some computations and sends a message back (which is possibly the unchanged ciphertext sent in the previous step). 
13. Attacker also sends the Challenger further possible encrypted signed ballots of the other, possibly corrupted voters.

14. Challenger waits for all the signed encrypted ballots to arrive. It decrypts the signed ballots with the secret key of the mixer, and checks if all the decrypted messages are distinct and are belong to the voting phase. If these checks succeeded, then the challenger puts them through the shuffle and gives the result of the shuffle to the attacker. Otherwise, the challenger aborts.

15. Attacker creates the bulletin board $\mathrm{BB}$ and gives it to the Challenger. Note that the ballots of $A$ and $B$ may or may not appear on the BB. Attacker also specifies which of $A$ or $B$ should move to the next step first.

16. If $A$ (resp. $B$ ) was asked to move next, $A$ (resp. $B$ ) accepted the blind signature in phase 2 and $A$ 's (resp. B's) ballot and the nonce appear on the bulletin board, then the Challenger sends $A$ 's (resp. B's) $\left\langle l_{i}, k_{i}\right.$, phase $\left._{3}\right\rangle$ to the attacker encrypted with the public key of the mixer. Otherwise, the challenger skips this step.

17. Attacker makes some computations and sends a message back (which is possibly the unchanged cipher sent in the previous step).

18. If the other agent accepted the blind signature in phase 2 and its ballot and the nonce appeared on the bulletin board, then the Challenger sends the other agent's $\left\langle l_{i}, k_{i}\right.$, phase $\left._{3}\right\rangle$ to the attacker encrypted with the public key of the mixer. Otherwise, it skips this step.

19. Attacker makes some computations and sends a message back (which is possibly the unchanged cipher sent in the previous step).

20. Attacker may also send other messages of the form $\left\langle l_{i}, k_{i}\right.$, phase $\left._{3}\right\rangle$ encrypted. 
21. Challenger decrypts the messages, checks if all the decrypted messages are distinct and are belong to the opening phase. The challenger also checks if both the commitment keys ( $A$ 's and $B$ 's) appear in the decrypted messages. If all these checks succeeded, Challenger then puts the decrypted messages through shuffle and gives the result of the shuffle to the attacker. Otherwise Challenger aborts.

22. The attacker outputs 0 or 1 .

If in the situation above the probability that the attacker outputs 1 playing game $\Pi_{0}$ is non-negligibly different from outputting 1 playing game $\Pi_{1}$, then the attacker wins the game. Our aim is to show that no PPT attacker can win the above game.

\subsubsection{Attacks on the FOO Protocol}

We describe four new attacks on the vote privacy for FOO protocol that we caught with the CCSA technique. The first two attacks cannot be captured in the DolevYao attacker model as they cannot be discovered under the assumption of perfect cryptography. Moreover, the first attack is an issue of the original FOO protocol and the standard definition of blindness property; it is not a feature of our implementation of anonymous channels. On the other hand, the second attack appears because our implementation uses CCA secure encryption. The third attack is Dolev-Yao attacker in nature but does not appear in previous Dolev-Yao analyses of the protocol as those analyses assume perfectly anonymous channels. Finally, we also point out a man-in-the-middle attack on the FOO protocol.

The first attack exploits an insufficiency in the blindness property. This insufficiency has also been pointed out in [FS09]. Recall that in the blindness game, a (potentially dishonest) signer engages in two sessions (parallel or sequential) with an honest user on messages $m_{0}$ and $m_{1}$. If one session successfully completes and the 
other aborts then the information of which session aborted is not revealed to the attacker. Thus, the blindness property does not rule out the possibility that the signer is able to deduce which session corresponded to $m_{0}$ and which corresponded to $m_{1}$ if one session aborted and the signer knows which session aborted. For example, in the three-step blind signature scheme described above, replace accept by accept ${ }^{\prime}$ where accept $^{\prime}\left(m, p k, r_{b}, \rho\right)$ always returns false if $m$ is of even length and returns the value of $\operatorname{accept}\left(m, p k, r_{b}, \rho\right)$ otherwise. The resulting blind signature scheme continues to satisfy the blindness property if the original one does. Now, if $m_{0}$ is of even length and $m_{1}$ is of odd length, then the session corresponding to message $m_{0}$ always aborts and thus the dishonest signer be able to associate the aborted session to $m_{0}$.

\subsubsection{A computational attack due to unequal vote lengths}

The first attack on FOO protocol proceeds as follows. Assume that we use the modified blind signature scheme in the FOO protocol and that the candidate choice $v_{0}$ is of even length and the choice $v_{1}$ is of odd length. Since commitments may reveal the length, it is possible that the commitment of $v_{0}$ is also even while of $v_{1}$ is odd. Assume that $A$ chooses $v_{b}$ and Bob chooses $v_{1-b}$. Vote privacy requires that the attacker not be able to deduce the value of $b$. In authentication phase, the attacker, acting as the administrator, correctly follows the protocol. The blinding still hides the commitments even though their lengths are different, but according to our modified accept algorithm, the signature on $v_{0}$ will be rejected while the one on $v_{1}$ will be accepted. Then during the voting phase, only one voter will send its ballot to the administrator. If this voter is $A$ then $b$ must be 1 , otherwise $b$ must be 0 . Note, this attack is not a feature of our specific implementation of the anonymous channel. Note also that even though the commitments are revealed on the $\mathrm{BB}$, there they appear in the same order on the two sides, so the attack works not because the commitments reveal to the attacker the vote inside, but because one accept may pass, the other may 
not. Observe also that the attack does not manifest if $v_{0}$ and $v_{1}$ are of equal length. Indeed, if $v_{0}$ and $v_{1}$ are of equal length then the property of commitment hiding ensures that an abort by a voter does not reveal information about its intended vote.

\subsubsection{A computational attack due to unequal blind signature lengths}

The second attack relies on the fact that there is no guarantee on the lengths of the (blind) signatures and that an encryption scheme used may reveal the lengths of the underlying plain-texts. If a length revealing encryption scheme (such as IND-CCA2) is used then the encrypted ballots in the voting phase are different, which allows the attacker to deduce how $A$ and $B$ voted. To rule out this attack, we have to assume that the (blind) signatures on equal length messages are of equal length.

\subsubsection{A Dolev-Yao style replay attack}

We point out the Dolev-Yao style attack on the FOO protocol. The attack replays a message in the voting phase of the protocol in the opening phase. Assume that there is a third voter $C$ in addition to $A$ and $B$. The attacker remembers $A$ 's encrypted ballot to the mixer in the voting phase. In the opening phase of the protocol, the attacker forwards the encrypted commitment keys of $A$ and $B$ to the mixer, but replaces $C$ 's commitment key by $A$ 's encrypted ballot from the voting phase. The mixer checks that the three encryptions are different, decrypts them and outputs them after shuffling them into lexicographic order. The only ballot that appears in the output is $A$ 's. The commitment keys of $A$ and $B$ are also part of the output. The attacker can then deduce how $A$ and $B$ voted. In order to prevent this attack, we modify the FOO protocol to also include phase numbers inside the encryptions in the voting and opening phases of the FOO protocol. The mixer must check that the phase numbers correspond to the current phase in the protocol. 


\subsubsection{A man-in-the-middle attack}

We also point out a man-in-the-middle attack on the FOO protocol. Assume that there are three voters in the protocol: two honest voters $A$ and $B$, and a dishonest voter $C$. In the voting phase, the dishonest voter $C$ intercepts one of the two ballots of the honest voters and sends some garbage to the mixer instead. Then in the opening phase, the attacker creates a bulletin board BB which contains all the ballots of the honest voters. We argue that this may happen because there is no assumption in the modeling that prevents the attacker in producing such BB. Unaware of this situation, the honest voters follow the protocol and reveal the respected keys in the opening phase. Of course, now the attacker can distinguish these two situations by creating the commitments of the other voter. In order to prevent this attack, in the voting phase, the honest voters must generate and include a nonce in addition to the phase number in the ballot.

Finally, we comment on how we found these attacks. We illustrate this on the first two attacks. Suppose that we do not require that the lengths of the names of the candidates are the same. At a certain point we have to verify that the messages sent by the challenger up to point 9 in Section 3.4.1 are equivalent in the two executions with flipped votes. This is the point when one of $A$ or $B$ according to the attacker's choice is supposed to have sent her ballot after having accepted the blind signature. As all the messages in the frames, this on both sides has the form of if $c$ then $t_{1}$ else $t_{2}$, where $c$ has all the conditions to get to this point, $t_{2}=\mathbf{0}$, and $t_{1}$ has the encrypted ballot of either $A$ or $B$ depending on which of them was called first by the adversary, which in turn contains the corresponding unflipped vote on one side and the flipped vote on the other side. To show that the two sides are equivalent, we need to use the INDCCA2 axiom for encryption, but that is only applicable if the equality of the lengths of the plaintexts are verified. However, without the formula $L\left(v_{1}\right)=L\left(v_{2}\right)$ it cannot be derived, and setting $L\left(v_{1}\right) \neq L\left(v_{2}\right)$ gives our second attack through an Herbrand 
model. The first attack is obtained when we try to verify that the condition $c$ on the two sides are equivalent. This condition contains the acc function symbol applied on the blind signature of $v_{1}$ or $v_{2}$, flipped on the two sides. The only axiom that is suitable to verify such difference (no encryption in $c$ ) is the hiding of the commitment. Note, the blindness axiom can only be applied once both blind signatures were accepted, but here we only have one on each branch. The hiding of the commitment on the other hand requires equal lengths. We can set again $L\left(v_{1}\right) \neq L\left(v_{2}\right)$, one of the accepts to true and the other to false, and again an Herbrand model delivers a symbolic attack, which also turns out to be a real computational one.

\subsection{Vote Privacy for the FOO Protocol in CCSA Framework}

We now explain how vote privacy for modified FOO protocol can be modeled in CCSA framework. We assume that the FOO protocol is modified to ensure that the length candidate identities are equal and that the phase identifiers are used in Voting and Opening phase. We will drop the adjective modified in the rest of the section. In order to make things simpler, we assume that other than voters $A$ and $B$, there is only a single other (possibly dishonest) voter. The proof of vote privacy carries over to $n$ voters for any $n$.

\subsubsection{Cryptographic Primitives and their Modeling}

In order to formalize the FOO voting protocol in the CCSA framework, we shall include the following (see the respective boxes) function symbols in $\mathcal{F}$. Each keygeneration algorithm and each random seed generation will be represented by a corresponding unary function symbol. When the function symbol is applied to a name $n$ (recall that names represent truly random objects), then the resulting term shall 
represent a correctly generated key or seed.

Commitments. We include a symbol $\mathrm{k}_{\mathrm{c}}(-)$ in $\mathcal{F}$ to represent the key generation algorithm for the commitment schemes defined in Section 3.3. com $(x, y)$ is the commitment of $x$ using the key $y$.

\begin{tabular}{|lrl|}
\hline $\mathrm{k}_{\mathrm{c}}\left({ }_{-}\right):$ & message $\rightarrow$ message \\
com $\left(_{-},{ }_{-}\right):$ & message $\times$message $\rightarrow$ message
\end{tabular}

Encryptions. The symbol $k_{\mathrm{e}}(-)$ denotes the public-key secret-key pair generation algorithm of encryptions. We define $\mathrm{pk}_{\mathrm{e}}(x) \stackrel{\text { def }}{\equiv} \pi_{1}\left(\mathrm{k}_{\mathrm{e}}(x)\right)$ and $\mathrm{sk}_{\mathrm{e}}(x) \stackrel{\text { def }}{\equiv} \pi_{2}\left(\mathrm{k}_{\mathrm{e}}(x)\right)$, the public encryption key and secret decryption key parts of $\mathrm{k}_{\mathrm{e}}(x)$ respectively. In order to formalize the random seed of encryptions, we introduce a symbol $r_{\mathrm{e}}\left({ }_{-}\right)$. Encryption of $x$ with random seed $z$ and public key $y$ is denoted as $\{x\}_{y}^{z}$ and decryption of $x$ with secret key $z$ is denoted as $\operatorname{dec}(x, z)$. We assume that for all $x, y, z$, $\operatorname{dec}\left(\{x\}_{\mathrm{pk}_{\mathrm{e}}(y)}^{z}, \operatorname{sk}_{\mathrm{e}}(y)\right)={ }_{E} x$.

\begin{tabular}{|lrl|}
\hline $\mathrm{k}_{\mathrm{e}}\left({ }_{-}\right):$ & message $\rightarrow$ message \\
$\mathrm{r}_{\mathrm{e}}\left({ }_{-}\right):$ & message $\rightarrow$ message \\
\{\}$_{-}^{-}:$ & message $\times$message $\times$message $\rightarrow$ message \\
$\operatorname{dec}\left({ }_{-},,_{-}:\right.$ & message $\times$message $\rightarrow$ message
\end{tabular}

Blind signatures. We introduce a symbol $\mathrm{k}_{\mathrm{b}}(-)$ to represent a public-verificationkey secret-signing-key pair generation algorithm of blind signatures. Once again, $\mathrm{pk}_{\mathrm{b}}(x) \stackrel{\text { def }}{\equiv} \pi_{1}\left(\mathrm{k}_{\mathrm{b}}(x)\right)$ and $\mathbf{s k}_{\mathrm{b}}(x) \stackrel{\text { def }}{\equiv} \pi_{2}\left(\mathrm{k}_{\mathrm{b}}(x)\right)$ represent the public verification key and secret signing key parts of the key $\mathrm{k}_{\mathrm{b}}(x)$. We also introduce a symbol $\mathrm{r}_{\mathrm{b}}(-)$ to represent blinding key generation algorithm. The interpretations of the function symbols b, bsign, bver, ub and acc are blind, blindsign, verify, unblind and accept as defined in Section 3.3. $\mathrm{b}(x, y, z)$ is the blinding of the message $x$ using the public 
verification key $y$ and the blinding key $z$. bsign $(x, y, r)$ is the (blinded) message $x$ blind-signed with secret blind signing key $y$ and random $\operatorname{seed} r \cdot \operatorname{acc}(x, y, z, w)$ is the acceptance check of the blindsign $w$ created for the blinded message $\mathrm{b}(x, y, z)$ and $\mathrm{ub}(x, y, z, w)$ its unblinding. $\perp$ represents undefined that is used to model blindness game. Finally, bver $(x, s, y)$ is the verification of unblinded signature $s$ on the message $x$ with the public verification key $y$. The co-domains of the symbol acc and bver are bool.

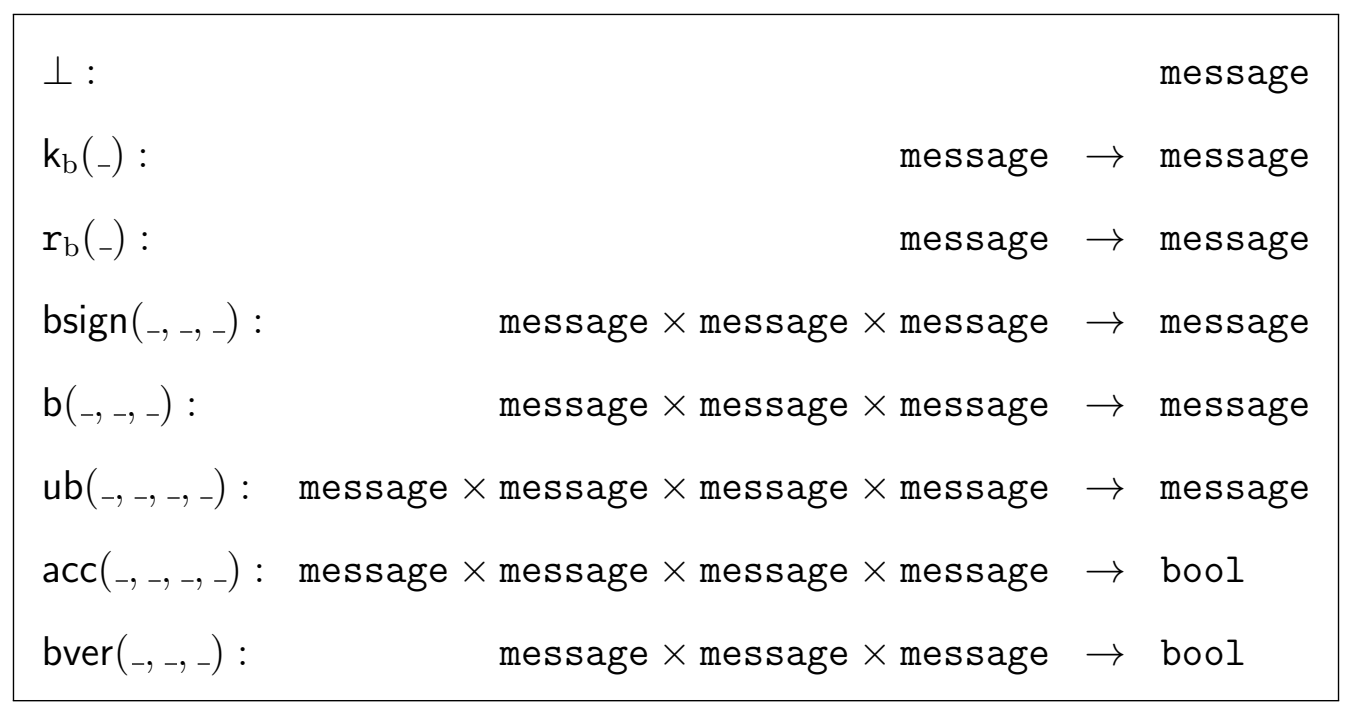

Digital signatures. In addition to blind signatures, we also need plain digital signatures. As in [BCEO19], let $\mathrm{k}_{\mathrm{s}}(-)$ represent generation of a key pair, the public verification key and the secret signing key. $\mathbf{v k}(x) \stackrel{\text { def }}{=} \pi_{1}\left(\mathrm{k}_{\mathrm{s}}(x)\right)$ and $\operatorname{ssk}(x) \stackrel{\text { def }}{=} \pi_{2}\left(\mathrm{k}_{\mathrm{s}}(x)\right)$ represent the public verification key and secret signing key parts of the key $\mathrm{k}_{\mathrm{s}}(x)$. In order to randomize signatures, we introduce a symbol $r_{s}(-)$ for random seed generation. $\operatorname{sign}(x, y, r)$ is the message $x$ singed with secret signing key $y$ with a random seed $r$ and $\operatorname{ver}(z, u, y)$ is the verification of signature $u$ on the message $z$ with the 
public verification key $y$.

\begin{tabular}{|lrl}
\hline $\mathrm{k}_{\mathrm{s}}\left(\left(_{-}\right):\right.$ & message $\rightarrow$ message \\
$\mathrm{r}_{\mathrm{S}}\left(\left(_{-}\right):\right.$ & message $\rightarrow$ message \\
$\operatorname{sign}\left({ }_{-},,_{-}\right):$ & message $\times$message $\times$message $\rightarrow$ message \\
$\operatorname{ver}\left({ }_{-},,_{-}\right):$ & message $\times$message $\times$message $\rightarrow$ bool
\end{tabular}

Mixer. We introduce a symbol shufl(-,,,$\left._{-}\right)$to model the shuffling functionality of mix-nets. shufl $\left(x_{1}, x_{2}, x_{3}\right)$ is the lexicographic ordering of the messages $x_{1}, x_{2}$, and $x_{3}$. As we model only three voters, shufl has only three arguments.

$$
\text { shufl }\left(,_{-},{ }_{-}\right): \text {message } \times \text { message } \times \text { message } \rightarrow \text { message }
$$

Axioms. We present axioms (see Figures 3.7, 3.8, 3.9, and 3.10) that formalize the security assumptions on the primitives. The axioms use the notion of freshness [BCL14] and reproduced in 2.4.4.

The axiom CompHid models the computational hiding property of the commitments (see Section 3.3). Intuitively, the axiom says that if the attacker is presented with commitments on equal length messages but the order of these messages is hidden, then the attacker cannot determine the order if it does not have access to commitment keys. CompBind models the computational binding property of the commitments which states that it is infeasible to produce two values $m$ and $m^{\prime}$ with $m \neq m^{\prime}$ such that $\operatorname{com}(m, k)=\operatorname{com}\left(m^{\prime}, k^{\prime}\right)$ for some values $k$ and $k^{\prime}$. CompHid and CompBind can be shown to be computationally valid if and only if $\llbracket k_{c}(-) \rrbracket$ and $\llbracket c o m\left({ }_{-},-\right) \rrbracket$ satisfy the computational hiding property. 
CompHid: Let $t, t_{1}$, and $t_{2}$ be three ground terms and let $\vec{z}$ be a list of ground terms and $n_{1}$ and $n_{2}$ be names such that $\operatorname{fresh}\left(n_{1}, n_{2} ; \vec{z}, t, t_{1}, t_{2}\right)$ holds.

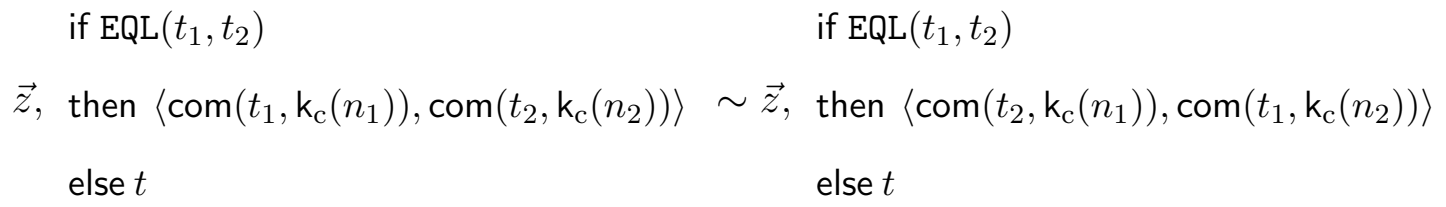

CompBind: Let $t_{1}, t_{2}, t_{3}$, and $t_{4}$ be ground terms.

$$
\mathrm{EQ}\left(\operatorname{com}\left(t_{1}, t_{3}\right), \operatorname{com}\left(t_{2}, t_{4}\right)\right)=\mathrm{EQ}\left(t_{1}, t_{2}\right)
$$

Figure 3.7: Axioms that formalize the computational assumptions of the commitments

The axiom BLindness models the blindness property of the blind signatures (see Section 3.3). Intuitively, in the blindness game, the dishonest signer attacker is asked to sign two blinded messages (could be of unequal length), but the order of these messages is hidden. Thus, the blinded messages on the two sides of $\sim$ are in reversed order. Moreover, the signer is also allowed to see the unblinded signatures if the user does not abort for either signature. However, in order to hide the order, the unblinded signatures are presented in the same order. BLINDNESS can be shown to be computationally valid if and only if blind signature scheme $\left(\llbracket k_{b} \rrbracket \cdot \llbracket r_{b} \rrbracket, \llbracket b \rrbracket, \llbracket u b \rrbracket, \llbracket b s i g n \rrbracket, \llbracket a c c \rrbracket, \llbracket b v e r \rrbracket\right)$ satisfies the blindness property. 
Blindness: Let $m_{0}, m_{1}, t$ be ground terms and let $\vec{z}$ be a list of ground terms. Let $t_{0}$ and $t_{1}$ be terms containing two variables $x$ and $y$. Let $n_{0}$, and $n_{1}$ be names such that fresh $\left(n_{0}, n_{1} ; \vec{z}, t, m_{0}, m_{1}, t_{0}, t_{1}\right)$ holds.

$\vec{z}, \mathrm{~b}\left(m_{0}, t, \mathrm{r}_{\mathrm{b}}\left(n_{0}\right)\right), \mathrm{b}\left(m_{1}, t, \mathrm{r}_{\mathrm{b}}\left(n_{1}\right)\right), u^{0} \sim \vec{z}, \mathrm{~b}\left(m_{1}, t, \mathrm{r}_{\mathrm{b}}\left(n_{0}\right)\right), \mathrm{b}\left(m_{0}, t, \mathrm{r}_{\mathrm{b}}\left(n_{1}\right)\right), u^{1}$

where $u^{j}=$ if $\operatorname{acc}_{0}^{j} \& \operatorname{acc}_{1}^{j}$ then $p^{j}$ else $\langle\perp, \perp\rangle, \operatorname{acc}_{i}^{0}=\operatorname{acc}\left(m_{i}, t, \Upsilon_{\mathrm{b}}\left(n_{i}\right), t_{i}^{0}\right)$

$\operatorname{acc}_{i}^{1}=\operatorname{acc}\left(m_{1-i}, t, \mathrm{r}_{\mathrm{b}}\left(n_{i}\right), t_{i}^{1}\right), p^{\mathrm{j}}=\left\langle\mathrm{ub}\left(m_{0}, t, \mathrm{r}_{\mathrm{b}}\left(n_{j}\right), t_{j}^{\mathrm{j}}\right), \mathrm{ub}\left(m_{1}, t, \mathrm{r}_{\mathrm{b}}\left(n_{1-j}\right), t_{1-j}^{\mathrm{j}}\right)\right\rangle$

$t_{i}^{\mathrm{j}}=t_{i}\left[x \leftarrow \mathrm{b}\left(m_{j}, t, \mathrm{r}_{\mathrm{b}}\left(n_{0}\right)\right), y \leftarrow \mathrm{b}\left(m_{1-j}, t, \mathrm{r}_{\mathrm{b}}\left(n_{1}\right)\right)\right], i, j \in\{0,1\}$

Figure 3.8: Axiom that formalizes the blindness property of the Blind Signatures

The axiom SHuffle models the shuffling capability of the mixer.

Shuffle: For any permutation $p$ of $\{1,2,3\}, \operatorname{shufl}\left(x_{1}, x_{2}, x_{3}\right)=\operatorname{shufl}\left(x_{p(1)}, x_{p(2)}, x_{p(3)}\right)$

Figure 3.9: SHuFfle axiom

Finally, we recall the axiom representing IND-CCA2 property of encryptions from [BCEO19]. Let $\vec{t}[x]$ be a list of terms with a single variable $x$. For a closed term $v$, let $\vec{t}[v]$ denote the term that we receive from $\vec{t}[x]$ by replacing all occurrences of $x$ by $v$. Let $u, u^{\prime}, u^{\prime \prime}$ be closed terms. 
Consider the formula

$\vec{t}\left[\right.$ if $\operatorname{EQL}\left(u, u^{\prime}\right)$ then $\{u\}_{\mathrm{pk}_{\mathrm{e}}\left(n_{1}\right)}^{\mathrm{r}_{\mathrm{e}}\left(n_{2}\right)}$ else $\left.u^{\prime \prime}\right] \sim \vec{t}\left[\right.$ if $\operatorname{EQL}\left(u, u^{\prime}\right)$ then $\left\{u^{\prime}\right\}_{\mathrm{pk}_{\mathrm{e}}\left(n_{1}\right)}^{\mathrm{r}_{\mathrm{e}}\left(n_{3}\right)}$ else $\left.u^{\prime \prime}\right]$

where

- $n_{1} \in \mathcal{N}$ occurs only as $\mathrm{k}_{\mathrm{e}}\left(n_{1}\right)$, sk $\mathrm{s}_{\mathrm{e}}\left(n_{1}\right)$ only occurs in decryption position (that is, as in $\left.\operatorname{dec}\left({ }_{-}, \mathbf{s k}_{\mathrm{e}}\left(n_{1}\right)\right)\right)$ and $n_{2}, n_{3}$ do not occur anywhere else

- We call the above formula $\operatorname{ENC}_{\mathrm{CCA} 2}$ if for any $t^{\prime}[x]$ term with $x$ explicitly occurring in $t^{\prime}[x], \operatorname{dec}\left(t^{\prime}[x], \mathrm{sk}_{\mathrm{e}}\left(n_{1}\right)\right)$ occurs only as if $\mathrm{EQ}\left(t^{\prime}[x], x\right)$ then $t^{\prime \prime}[x]$ else $\operatorname{dec}\left(t^{\prime}[x], \mathrm{sk}_{\mathrm{e}}\left(n_{1}\right)\right)$, where $t^{\prime \prime}[x]$ is not of the form $\operatorname{dec}\left(t^{\prime \prime \prime}[x], \mathrm{sk}_{\mathrm{e}}\left(n_{1}\right)\right)$

Figure 3.10: Modeling IND-CCA2 of encryptions in the CCSA framework

The intuition is that since in the IND-CCA2 game, after encryption, the decryption oracle decrypts only those messages that are different from the encryption, we have to make sure that the decrypted message, $t^{\prime}[x]$ is different from the encryption, for which $x$ stands. The reader is referred to [BCEO19] for additional details.

\subsubsection{Proofs of Validity}

Proposition 3.8 The CompHid axiom is computationally valid if and only if $\llbracket k_{\mathrm{c}}\left({ }_{-}\right) \rrbracket$ and $\llbracket \operatorname{com}\left({ }_{-},-\right) \rrbracket$ satisfy the computational hiding property.

Proof Sketch: For terms $t_{1}, t_{2}$, let $u^{\text {left }}$ and $u^{\text {right }}$ be the pairs $\operatorname{com}\left(t_{1}, \mathrm{k}_{\mathrm{c}}\left(n_{1}\right)\right)$, $\operatorname{com}\left(t_{2}, \mathbf{k}_{\mathrm{c}}\left(n_{2}\right)\right)$ and $\operatorname{com}\left(t_{2}, \mathbf{k}_{\mathrm{c}}\left(n_{1}\right)\right), \operatorname{com}\left(t_{1}, \mathbf{k}_{\mathrm{c}}\left(n_{2}\right)\right)$ respectively.

Assume that there are closed terms $t_{1}, t_{2}$, a list of closed terms $\vec{z}$ such that $\operatorname{fresh}\left(n_{1} ; \vec{z}, t_{1}, t_{2}\right)$ and $\operatorname{fresh}\left(n_{2} ; \vec{z}, t_{1}, t_{2}\right)$ hold, and a computational model $\mathcal{M}^{c}$ such that

$$
\mathcal{M}^{c} \not \vec{z}, u^{\text {left }} \sim \vec{z}, u^{\text {right }}
$$


This means that there is a PPT machine $\mathcal{A}$ such that

$$
\left|\operatorname{Pr}\left\{\rho: \mathcal{A}\left(\llbracket \vec{z}, u^{\text {left }} \rrbracket_{\eta, \rho} ; \rho_{2}\right)=1\right\}-\operatorname{Pr}\left\{\rho: \mathcal{A}\left(\llbracket \vec{z}, u^{\text {right }} \rrbracket_{\eta, \rho} ; \rho_{2}\right)=1\right\}\right|
$$

is non-negligible in $\eta$. By the freshness conditions, $\vec{z}$ can also be computed by the attacker, hence there is an $\mathcal{A}$ such that

$$
\begin{aligned}
& \mid \operatorname{Pr}\left\{\rho: \mathcal{A}\left(\llbracket \operatorname{com}\left(t_{1}, \mathrm{k}_{\mathrm{c}}\left(n_{1}\right)\right), \operatorname{com}\left(t_{2}, \mathrm{k}_{\mathrm{c}}\left(n_{2}\right)\right) \rrbracket_{\eta, \rho} ; \rho_{2}\right)=1\right\}- \\
& \operatorname{Pr}\left\{\rho: \mathcal{A}\left(\llbracket \operatorname{com}\left(t_{2}, \mathrm{k}_{\mathrm{c}}\left(n_{1}\right)\right), \operatorname{com}\left(t_{1}, \mathrm{k}_{\mathrm{c}}\left(n_{2}\right)\right) \rrbracket_{\eta, \rho} ; \rho_{2}\right)=1\right\} \mid
\end{aligned}
$$

is non-negligible and it is clearly violates the computational hiding property. In the other direction, let $f_{0}, f_{1} \in \mathcal{G}$ be zero-arity adversarial function symbols. Let $\mathcal{M}^{c}$ be such that it interprets the names to have uniform distributions over $\{0,1\}^{p(\eta)}$ for some large enough polynomial $p$, and $\llbracket k_{c} \rrbracket$ some algorithm that outputs a key for an input in $\{0,1\}^{p(\eta)}$. If there are $\llbracket \mathbf{k}_{\mathrm{c}} \rrbracket, m_{0}$ and $m_{1}$, such that

$$
\begin{aligned}
& \mid \operatorname{Pr}\left\{\rho: \mathcal{A}\left(\llbracket \operatorname{com}\left(m_{0}, \mathrm{k}_{\mathrm{c}}\left(n_{0}\right)\right), \operatorname{com}\left(m_{1}, \mathrm{k}_{\mathrm{c}}\left(n_{1}\right)\right) \rrbracket_{\eta, \rho} ; \rho_{2}\right)=1\right\}- \\
& \operatorname{Pr}\left\{\rho: \mathcal{A}\left(\llbracket \operatorname{com}\left(m_{1}, \mathrm{k}_{\mathrm{c}}\left(n_{0}\right)\right), \operatorname{com}\left(m_{0}, \mathrm{k}_{\mathrm{c}}\left(n_{1}\right)\right) \rrbracket_{\eta, \rho} ; \rho_{2}\right)=1\right\} \mid
\end{aligned}
$$

is not negligible (where $\mathbf{k}_{\mathrm{c}}\left(n_{0}\right), \mathbf{k}_{\mathrm{c}}\left(n_{1}\right)$ are independently generated keys by $\llbracket \mathrm{k}_{\mathrm{c}} \rrbracket$ ), then further setting the interpretations of $f_{0}$ and $f_{1}$ in $\mathcal{M}^{c}$ to be those $m_{0}$ and $m_{1}$, we have that $\mathcal{M}^{c} \not \models u^{\text {left }} \sim u^{\text {right }}$.

Proposition 3.9 The CompBind axiom is computationally valid if $\llbracket k_{\mathrm{c}}(-) \rrbracket$ and $\llbracket \operatorname{com}(,,-) \rrbracket$ satisfy the computational binding property.

Proof Sketch: Assume that there are ground terms $t_{1}, t_{2}, t_{3}$ and $t_{4}$ and a computational model $\mathcal{M}^{c}$ such that $\mathrm{EQ}\left(\operatorname{com}\left(t_{1}, t_{3}\right), \operatorname{com}\left(t_{2}, t_{4}\right)\right)$ evaluates true while $\mathrm{EQ}\left(t_{1}, t_{2}\right)$ evaluates false or vice-versa. This means that $t$ rue $=$ false . 
However, by the definition of equality $=$, we have that $\mathrm{EQ}(\boldsymbol{t r u e}, \boldsymbol{f a l s e}) \sim \boldsymbol{t r u e}$. By theorem 9 in Chapter 4, we know that $\mathrm{EQ}(\boldsymbol{t r u e}$, false $)=$ false. Rewriting the term $\mathrm{EQ}(\boldsymbol{t r u e}$, false $)$, we get the equivalence false $\sim \boldsymbol{t r u e}$ which contradicts the TFDIST axiom.

Proposition 3.10 The Blindness axiom is valid if and only if the blind signature scheme $\left(\llbracket k_{b} \rrbracket . \llbracket r_{b} \rrbracket, \llbracket b \rrbracket, \llbracket u b \rrbracket, \llbracket b s i g n \rrbracket, \llbracket a c c \rrbracket, \llbracket b v e r \rrbracket\right)$ satisfies the blindness property.

Proof Sketch: Let us introduce the following notation:

$$
b_{i}^{j}\left[m_{i \oplus j}\right] \equiv \mathrm{b}\left(m_{i \oplus j}, t, \mathrm{r}_{\mathrm{b}}\left(n_{i}\right)\right) .
$$

Assume that there is a computational model $\mathcal{M}^{c}$ such that

$$
\mathcal{M}^{c} \not \forall \vec{z}, b_{0}^{0}\left[m_{0}\right], b_{0}^{1}\left[m_{1}\right], u^{0} \sim \vec{z}, b_{0}^{1}\left[m_{1}\right], b_{1}^{1}\left[m_{0}\right], u^{1}
$$

Where $u^{0}$ and $u^{0}$ are defined as in the BLInDness axiom. This means that there is a probabilistic polynomial-time Turing machine $\mathcal{A}$ such that

$$
\left|\operatorname{Pr}\left\{\rho: \mathcal{A}\left(\llbracket \vec{z}, b_{0}^{0}\left[m_{0}\right], b_{0}^{1}\left[m_{1}\right], u^{0} \rrbracket_{\rho, \eta} ; \rho_{2}\right)=1\right\}-\operatorname{Pr}\left\{\rho: \mathcal{A}\left(\llbracket \vec{z}, b_{0}^{1}\left[m_{1}\right], b_{1}^{1}\left[m_{0}\right], u^{1} \rrbracket_{\rho, \eta} ; \rho_{2}\right)=1\right\}\right|
$$

is non-negligible function in $\eta$. Then an adversary $\mathcal{B}$ can win the blindness game as follows. According to the blindness definition, on the input $1^{\eta}$, the adversary $\mathcal{B}$ generates $p k$, which in this case shall be $\llbracket t \rrbracket_{\rho, \eta}$, and two messages $\llbracket m_{0} \rrbracket_{\rho, \eta}$ and $\llbracket m_{1} \rrbracket_{\rho, \eta}$. The adversary $\mathcal{B}$ also generates $\llbracket \vec{z} \rrbracket_{\rho, \eta}$. Then $\mathcal{B}$, gives $\llbracket t \rrbracket_{\rho, \eta}, \llbracket m_{0} \rrbracket_{\rho, \eta}, \llbracket m_{1} \rrbracket_{\rho, \eta}$ to the challenger as the blindness game requires. The challenger flips a coin to obtain the bit $b$, computes $\llbracket n_{0} \rrbracket_{\rho, \eta}, \llbracket n_{1} \rrbracket_{\rho, \eta}$, and then $\llbracket \mathrm{b}\left(m_{b}, t, \mathrm{r}_{\mathrm{b}}\left(n_{0}\right)\right), \mathrm{b}\left(m_{1-b}, t, \mathrm{r}_{\mathrm{b}}\left(n_{1}\right)\right) \rrbracket_{\rho, \eta}$, and gives it to the attacker. The attacker than substitutes these to $x$ and $y$ in $t_{0}$ and $t_{1}$, generates the necessary further items in these terms, and depending on the value of $b$, computes $\llbracket t_{0}^{0} \rrbracket_{\rho, \eta}$ and $\llbracket t_{1}^{0} \rrbracket_{\rho, \eta}$ or $\llbracket t_{1}^{0} \rrbracket_{\rho, \eta}$ and $\llbracket t_{1}^{1} \rrbracket_{\rho, \eta}$,

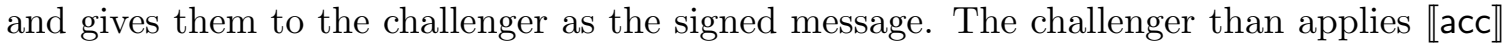


on them, and when it outputs 1 in both cases, the challenger unblinds the signatures and produces either $\llbracket u^{0} \rrbracket$ or $\left.\llbracket u^{1} \rrbracket\right)$ depending on the value of $b$ and gives it to the attacker $\mathcal{B}$. $\mathcal{B}$ then passes all the messages it computed and obtained from the challenger to $\mathcal{A}$, which, because of our assumption breaks the security game.

Which implies that $\operatorname{Pr}\left\{\llbracket b_{0}^{0}\left[m_{0}\right], b_{0}^{1}\left[m_{1}\right], u \rrbracket \neq \llbracket b_{0}^{1}\left[m_{1}\right], b_{1}^{1}\left[m_{0}\right], u^{\prime} \rrbracket\right\}$ is non-negligible function in $\eta$.

Now, we show that a malicious signer $\mathcal{B}$ can win the game $\prod_{\tilde{b}}^{\text {blindness }}$ as follows. On the given security parameter $\eta, \mathcal{B}$ can generate a public and private key pair $\left\langle\llbracket p k(n) \rrbracket_{\rho, \eta}, \llbracket s k(n) \rrbracket_{\rho, \eta}\right\rangle$. The adversary $\mathcal{B}$ interprets the names that occur in $\vec{u}, m_{0}, m_{1}, u^{\prime}, u$ according to $\mathcal{M}^{c}$. $\mathcal{B}$ generates $m_{0}$ and $m_{1}$ of length polynomial in $\eta$, and then engage in concurrent sessions with $U \operatorname{ser}\left(\mathrm{pk}_{\mathrm{b}}(n), m_{b}\right)$ and $U \operatorname{ser}\left(\mathrm{pk}_{\mathrm{b}}(n), m_{1-b}\right)$, where $b$ is randomly chosen from $\{0,1\}$ and kept secret from the adversary. $\mathcal{B}$ passes the interpretations of the terms $\vec{u}, m_{0}, m_{1}, \mathrm{~b}\left(m_{b}, \mathrm{pk}_{\mathrm{b}}(n), \mathrm{r}_{\mathrm{b}}\left(n_{1}\right), \mathrm{b}\left(m_{1-b}, \mathrm{pk}_{\mathrm{b}}(n), \mathrm{r}_{\mathrm{b}}\left(n_{2}\right), u, u^{\prime}\right.\right.$ to $\mathcal{A}$. Then $\mathcal{B}$ outputs the output of $\mathcal{A}$. Hence,

$$
\left.\operatorname{Pr}\left\{\mathcal{B}\left(1^{\eta}\right)=1, b=0\right)\right\}=\operatorname{Pr}\left\{\rho: \mathcal{A}\left(\llbracket \vec{u}, m_{0}, m_{1}, \mathrm{~b}\left(m_{0}, \mathrm{pk}_{\mathrm{b}}(n), b_{0}^{0}\left[m_{0}\right], b_{0}^{1}\left[m_{1}\right], u \rrbracket_{\rho, \eta} ; \rho_{2}\right)=1\right\}\right.
$$

and $\left.\operatorname{Pr}\left\{\mathcal{B}\left(1^{\eta}\right)=1, b=1\right)\right\}=\operatorname{Pr}\left\{\rho: \mathcal{A}\left(\llbracket \vec{u}, m_{0}, m_{1}, b_{0}^{1}\left[m_{1}\right], b_{1}^{1}\left[m_{0}\right], u^{\prime} \rrbracket_{\rho, \eta} ; \rho_{2}\right)=1\right\}$. Since $A d v^{\mathcal{A}}(\eta)$ is non-negligible, we conclude that

$\left.\left.\operatorname{Pr}\left\{\mathcal{B}\left(1^{\eta}\right)=1, b=0\right)\right\}-\operatorname{Pr}\left\{\mathcal{B}\left(1^{\eta}\right)=1, b=1\right)\right\}$ is non-negligible. Now, we see that,

$$
\begin{aligned}
\left.\operatorname{Pr}\left\{\mathcal{B}\left(1^{\eta}\right)=1, b\right)\right\} & \left.\left.=\operatorname{Pr}\left\{\mathcal{B}\left(1^{\eta}\right)=1, b=1\right)\right\}+\operatorname{Pr}\left\{\mathcal{B}\left(1^{\eta}\right)=0, b=0\right)\right\} \\
& \left.\left.=\operatorname{Pr}\left\{\mathcal{B}\left(1^{\eta}\right)=1, b=1\right)\right\}+\left(\frac{1}{2}-\operatorname{Pr}\left\{\mathcal{B}\left(1^{\eta}\right)=1, b=0\right)\right\}\right) .
\end{aligned}
$$

The advantage of the signer $\mathcal{B}$ is given by,

$$
\begin{aligned}
A d v^{\mathcal{B}}(\eta) & \left.=\operatorname{Pr}\left\{\mathcal{B}\left(1^{\eta}\right)=1, b\right)\right\}-\frac{1}{2} \\
& \left.\left.=\operatorname{Pr}\left\{\mathcal{B}\left(1^{\eta}\right)=1, b=1\right)\right\}-\operatorname{Pr}\left\{\mathcal{B}\left(1^{\eta}\right)=1, b=0\right)\right\}
\end{aligned}
$$

is non-negligible, and BLINDNESS is broken by $\mathcal{B}$. 
The proof of validity of $\mathrm{ENC}_{\mathrm{CCA} 2}$ has been presented in [BCEO19].

\subsubsection{Other Function Symbols and Assumptions}

We introduce constants $\mathrm{ph}_{2}$ (resp. $\mathrm{ph}_{3}$ ) to represent the Voting (resp. Opening) Phase of the FOO protocol. These symbols are necessary to avoid replay attacks in which an attacker can learn honest vote value by forwarding one of the honest voter's message from the Voting Phase to the Opening Phase (see 3.4.2). Constants $A$ and $B$ model the identity of two honest voters and constant $M$ models the identity of the mixer. $C_{1}, C_{2}, C_{3}$ model the identities of the candidates (for simplicity, we assume only three candidates). Finally, the function symbols to(_), $\mathrm{V}_{0}(-), \mathrm{V}_{1}(-)$, pubkey(_) are used to model the security game and explained in Section 3.5.4.

\begin{tabular}{|llr|}
\hline $\mathrm{ph}_{2}, \mathrm{ph}_{3}:$ & & message \\
$A, B, M:$ & & message \\
$C_{1}, C_{2}, C_{3}:$ & & message \\
to(_) $:$ & message $\rightarrow$ & message \\
$\mathrm{V}_{0}\left({ }_{-}\right), \mathrm{V}_{1}\left({ }_{-}\right):$ & message $\rightarrow$ & message \\
pubkey(_) $:$ & message $\rightarrow$ & message \\
\hline
\end{tabular}

We also require further assumptions on the primitives. In particular, the commitments and pairing should be length regular, unblinded signatures on equal length messages by the same signer must have equal length, honestly generated commitment keys must have equal length, candidate names should have identical length and phase identifiers should be distinct but have identical length. 
Msgneql : For any ground term $t, \mathrm{EQ}(t, \operatorname{compl}(t)) \sim$ false.

\section{Commitments}

Let $t_{1}$ and $t_{2}$ be two ground terms. Let $n_{1}$ and $n_{2}$ be names such that fresh $\left(n_{1}, n_{2} ; t_{1}, t_{2}\right)$ holds. CommEQL: if $\operatorname{EQL}\left(t_{1}, t_{2}\right)$ then $\operatorname{EQL}\left(\operatorname{com}\left(t_{1}, \mathrm{k}_{\mathrm{c}}\left(n_{1}\right)\right), \operatorname{com}\left(t_{2}, \mathrm{k}_{\mathrm{c}}\left(n_{2}\right)\right)\right)$ else $\boldsymbol{t r u e}=\boldsymbol{t r u e}$ CommKeyEQL: $\operatorname{EQL}\left(\mathrm{k}_{\mathrm{c}}\left(n_{1}\right), \mathrm{k}_{\mathrm{c}}\left(n_{2}\right)\right)=$ true

\section{Blind Digital Signatures}

For $i=0,1$, let $t, t_{1}^{i}$, and $t_{2}^{i}$ be ground terms. Let $n^{i}$ be a name with fresh $\left(n^{i} ; t, t_{1}^{i}, t_{2}^{i}\right)$, such that it occurs in $t_{2}^{i}$, only as $\mathrm{b}\left(t_{1}^{i}, t, \mathrm{r}_{\mathrm{b}}\left(n^{i}\right)\right)$ :

UBNotUndefined:

if $\operatorname{acc}\left(t_{1}^{0}, t, \mathrm{r}_{\mathrm{b}}\left(n^{0}\right), t_{2}^{0}\right)$ then $\mathrm{EQ}\left(\mathrm{ub}\left(t_{1}^{0}, t, \mathrm{r}_{\mathrm{b}}\left(n^{0}\right), t_{2}^{0}\right), \perp\right)$ else false $=$ false $\mathrm{UBEQL}^{a}$ :

$$
\begin{aligned}
& \text { if } \operatorname{EQL}\left(t_{1}^{0}, t_{1}^{1}\right) \& \operatorname{acc}\left(t_{1}^{0}, t, \mathrm{r}_{\mathrm{b}}\left(n^{0}\right), t_{2}^{0}\right) \& \operatorname{acc}\left(t_{1}^{1}, t, \mathrm{r}_{\mathrm{b}}\left(n^{1}\right), t_{2}^{1}\right) \\
& \text { then } \operatorname{EQL}\left(\mathrm{ub}\left(t_{1}^{0}, t, \mathrm{r}_{\mathrm{b}}\left(n^{0}\right), t_{2}^{0}\right), \mathrm{ub}\left(t_{1}^{1}, t, \mathrm{r}_{\mathrm{b}}\left(n^{1}\right), t_{2}^{1}\right)\right)=\text { true } \\
& \text { else } \boldsymbol{t r u e}
\end{aligned}
$$

\section{Further length regularity}

PairEQL: if $\operatorname{EQL}\left(x_{1}, y_{1}\right)$ \& $\mathrm{EQL}\left(x_{2}, y_{2}\right)$ then $\mathrm{EQL}\left(\left\langle x_{1}, x_{2}\right\rangle,\left\langle y_{1}, y_{2}\right\rangle\right)$ else true = true CandEQL: $\operatorname{EQL}\left(C_{i}, C_{j}\right)=$ true for $i, j \in\{1,2,3\}$

PhaseEQL: EQL $\left(\mathrm{ph}_{2}, \mathrm{ph}_{3}\right)=$ true

ZEQL : $\operatorname{EQL}(\mathrm{z}(x), x)=$ true

\section{Distinctness of constants}

$$
\begin{aligned}
& \text { AgentDist: } \mathrm{EQ}(A, B)=\mathrm{EQ}(A, M)=\mathrm{EQ}(B, M)=\text { false } \\
& \text { PhaseDist: } \mathrm{EQ}\left(\mathrm{ph}_{2}, \mathrm{ph}_{3}\right)=\text { false }
\end{aligned}
$$

\section{Name length Equality}

NameEQL: For any two names $n_{0}$ and $n_{1}$, we have EQL $\left(n_{0}, n_{1}\right)=$ true

${ }^{a} \operatorname{In}[\mathrm{BCE} 18]$, we also check EQL $\left(t_{2}^{0}, t_{2}^{1}\right)$ which makes the axiom stronger. We weaken the axiom here by dropping it

Table 3.1: Axioms that formalize the additional assumptions of the primitives

Additional axioms used in the security game for the FOO protocol are given in Table 3.1. Msgneql models the fact that equality of a term and its complement is indistinguishable to false. CommEQL models the assumption that the length of commitments on equal length messages are equal. CommKeyEQL models the assumption that the length of honestly generated commitment keys are equal. UBNoTUNDEF INED 
models the assumption that if a user successfully completes the blind signature protocol then then the output is different from $\perp$. UBEQL models the assumption that blind signatures on equal length message are of equal length. PAIREQL models the length regularity of pairing. CANDEQL (resp. PhASEEQL) models the assumptions that candidate identities (resp. phase identities) are of equal length. ZEQL models the fact that for any term $t$, the lengths of $\mathbf{z}(t)$ and $t$ are equal. AgentDist (resp. PhaseDist) models the assumption that identities of honest voters (resp. phase identifiers) are distinct.

\subsubsection{Modeling the Vote Privacy Security Game}

As defined in [BCL14], the BC technique treats protocols as abstract transition systems. We do not recall the formal definitions, we just apply them to the vote privacy games $\Pi_{b}$ for $b=0,1$ between the Challenger and the Attacker as defined in Section 3.4.1. The transition system produces the terms of the execution, on which the indistinguishability predicate is applied. The verification task is to prove that the axioms together with first-order inference rules imply that the execution terms produced by the transition system for $b=0$ and for $b=1$ are indistinguishable.

Recall that in addition to honest voters $A$ and $B$, there is an additional voter only a single other voter. We assume the mixer $M$ is honest, and the administrator as well as the collector are corrupt. Recall that we assume that the names $A, B$, and $M$ are distinct message constants. We also use distinct names $n_{A}, n_{B}, n_{M}$ for (honest) signing key generations for the voters $A, B$, and encryption key generation for the Mixer $M$. The function symbol to extracts from an incoming message the agent name. This allows the attacker to specify which of $A, B$, and $M$ should receive the message sent by the attacker. The function symbols $\mathrm{V}_{b}, b \in\{0,1\}$ extract from an incoming attacker message the candidate choices for the honest voters $A$ and $B$. The function symbol pubkey extracts from an incoming attacker message the (dishonest) 


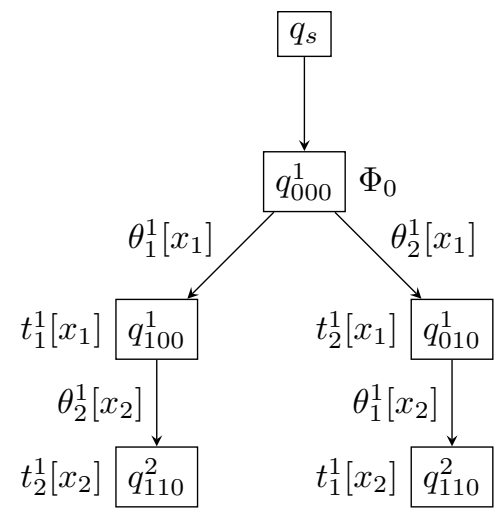

$$
\begin{aligned}
& \theta_{1}^{1}\left[x_{i}\right] \equiv\left(\mathrm{EQ}\left(\operatorname{to}\left(x_{i}\right), A\right) \& \operatorname{vcheck}\left[v_{b}\right] \& \operatorname{vcheck}\left[v_{1-b}\right]\right. \\
& t_{1}^{1}\left[x_{i}\right] \equiv\left\langle\operatorname{vk}\left(n_{A}\right), b_{1}, \operatorname{sign}\left(b_{1}, \operatorname{ssk}\left(n_{A}\right), \mathrm{r}_{\mathrm{s}}\left(n_{\mathrm{s}}^{1}\right)\right)\right\rangle \\
& \theta_{2}^{1}\left[x_{i}\right] \equiv\left(\mathrm{EQ}\left(\operatorname{to}\left(x_{i}\right), B\right) \& \operatorname{vcheck}\left[v_{1-b}\right] \& \operatorname{vcheck}\left[v_{b}\right]\right. \\
& t_{2}^{1}\left[x_{i}\right] \equiv\left\langle\operatorname{vk}\left(n_{B}\right), b_{2}, \operatorname{sign}\left(b_{2}, \operatorname{ssk}\left(n_{B}\right), \mathrm{r}_{\mathrm{s}}\left(n_{\mathrm{s}}^{2}\right)\right)\right\rangle \\
& \text { where } i \text { is in }\{1,2\} \\
& \Phi_{0} \equiv A, B, M, \operatorname{vk}\left(n_{A}\right), \operatorname{vk}\left(n_{B}\right), \operatorname{pk}_{\mathrm{e}}\left(n_{M}\right), C_{1}, C_{2}, C_{3} \\
& b_{1} \equiv \mathrm{b}\left(c_{1}, \operatorname{PK} \mathrm{AD}_{1}, \mathrm{r}_{\mathrm{b}}\left(n_{\mathrm{b}}^{1}\right)\right) ; c_{1} \equiv \operatorname{com}\left(v_{b}, \mathrm{k}_{\mathrm{c}}\left(n_{\mathrm{c}}^{1}\right)\right) \\
& b_{2} \equiv \mathrm{b}\left(c_{2}, \mathrm{PK} \mathrm{AD}_{\mathrm{AD}}, \mathrm{r}_{\mathrm{b}}\left(n_{\mathrm{b}}^{2}\right)\right) ; c_{2} \equiv \operatorname{com}\left(v_{1-b}, \mathrm{k}_{\mathrm{c}}\left(n_{\mathrm{c}}^{2}\right)\right) \\
& \operatorname{vcheck}[v] \equiv \mathrm{EQ}\left(v, C_{1}\right) \text { or EQ }\left(v, C_{2}\right) \text { or EQ }\left(v, C_{3}\right) \\
& v_{b} \equiv \mathrm{V}_{b}\left(x_{1}\right) ; v_{1-b} \equiv \mathrm{V}_{1-b}\left(x_{1}\right) \\
& \operatorname{PK}_{\mathrm{AD}} \equiv \operatorname{pubkey}\left(x_{1}\right)
\end{aligned}
$$

Figure 3.11: Authentication phase of the security game $\Pi_{b}$

public-key of the attacker.

We present the transition diagram that represents the Authentication Phase of the security game $\Pi_{b}$ in Figure 3.11. It illustrates all possible moves of the challenger in the authentication phase. At the start of the game, the knowledge of the attacker is initialized and is represented by $\phi_{0}$. The left branch of $q_{000}^{1}$ represents the situation where $A$ moves first for authentication followed by $B$ while the right branch simulates the situation that $B$ moves first followed by $A . \theta_{i}^{p}\left[x_{j}\right] \mathrm{s}$ are the bool conditions that the challenger checks upon receiving the message $x_{j}$ from the attacker before taking the respective transitions. Here $p$ represents the Phase number, $i$ represents the agent that has to move (we use 1 for $A, 2$ for $B$ and 3 for $M$ ) and $j$ represents the message number. Similarly the terms $t_{i}^{p}\left[x_{j}\right]$ s represent the outputs of the challenger to the attacker which increases attacker's knowledge. When the checks in a transition fail, the transition moves to state $q_{\text {exit }}$, which we omit in transition diagram for clarity.

The Voting Phase is in Figure 3.11 where we use similar conventions. It starts from the state $q_{110}^{2}$ which is final state of Authentication Phase. Again, the left branch simulates the situation where the voter $A$ receives message $x_{3}$ from an attacker and it is accepted as a blinded commitment which is signed by administrator. Then $A$ unblinds, encrypts the unblinded message with mixer's public key $\mathrm{k}_{\mathrm{e}}\left(n_{M}\right)$, and sends out the encrypted message $t_{1}^{2}\left[x_{3}\right]$. And so forth, following the security game. The abbreviation $\left\langle x_{1}, x_{2}, x_{3}\right\rangle \stackrel{\text { def }}{\equiv}\left\langle x_{1},\left\langle x_{2}, x_{3}\right\rangle\right\rangle$ encodes triples and $\tau_{1}(x) \stackrel{\text { def }}{\equiv} \pi_{1}(x), \tau_{2}(x) \stackrel{\text { def }}{\equiv}$ 

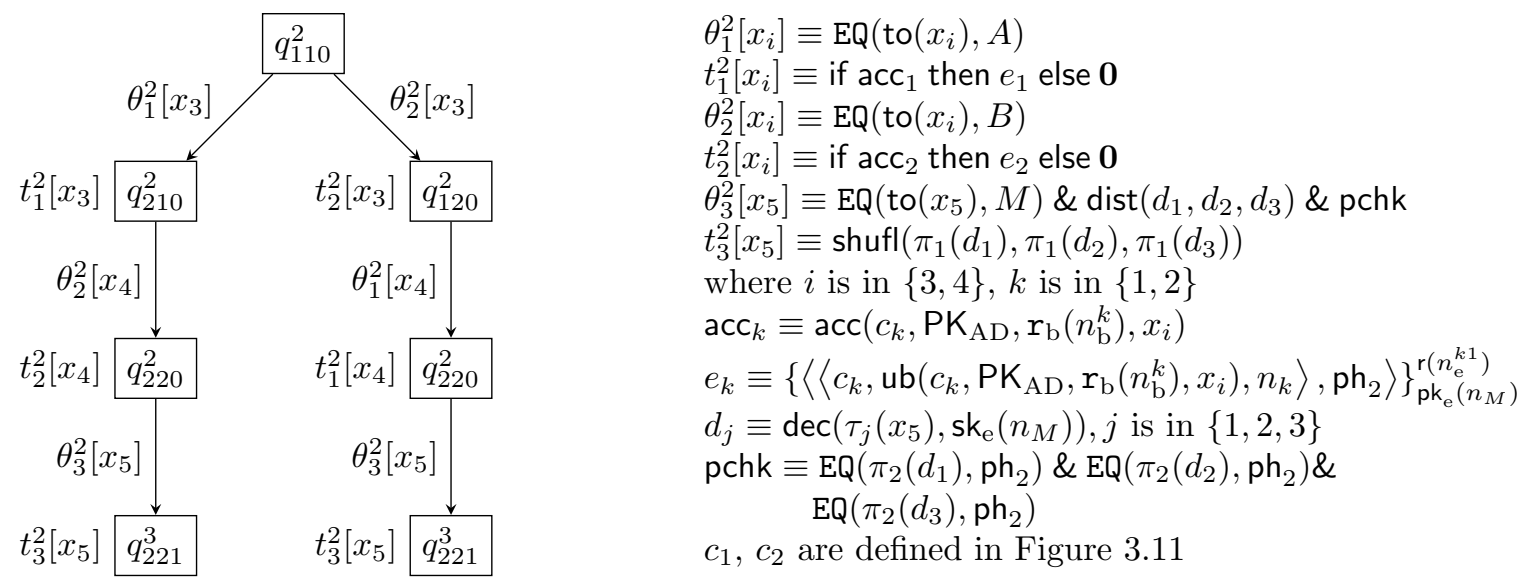

Figure 3.12: Voting Phase of the FOO protocol $\pi_{1}\left(\pi_{2}(x)\right)$ and $\tau_{3}(x) \stackrel{\text { def }}{=} \pi_{2}\left(\pi_{2}(x)\right)$ encode projections from triples.

In order to formalize the Opening Phase, we introduce the following abbreviations:

- $\operatorname{dist}(x, y, z) \stackrel{\text { def }}{\equiv} \operatorname{not}(\mathrm{EQ}(x, y)) \& \operatorname{not}(\mathrm{EQ}(x, z)) \& \operatorname{not}(\mathrm{EQ}(y, z))$.

- $\operatorname{isin}(x, y) \stackrel{\text { def }}{\equiv} \mathrm{EQ}\left(x, \tau_{1}(y)\right)$ or $\mathrm{EQ}\left(x, \tau_{2}(y)\right)$ or $\operatorname{EQ}\left(x, \tau_{3}(y)\right)$

- $\operatorname{bcheck}(x, y) \stackrel{\text { def }}{\equiv} \operatorname{isin}\left(x,\left\langle\tau_{1}\left(\pi_{2}\left(\tau_{1}(y)\right)\right), \tau_{1}\left(\pi_{2}\left(\tau_{2}(y)\right)\right), \tau_{1}\left(\pi_{2}\left(\tau_{3}(y)\right)\right)\right\rangle\right)$

- $\operatorname{ncheck}(x, y) \stackrel{\text { def }}{\equiv} \operatorname{isin}\left(x,\left\langle\tau_{3}\left(\pi_{2}\left(\tau_{1}(y)\right)\right), \tau_{3}\left(\pi_{2}\left(\tau_{2}(y)\right)\right), \tau_{3}\left(\pi_{2}\left(\tau_{3}(y)\right)\right)\right\rangle\right)$

- $\operatorname{bnlcheck}(x, y, z) \stackrel{\text { def }}{\equiv} \operatorname{bcheck}(x, z) \& \mathrm{~L}(\operatorname{label}(x, z))=\ell_{\mathrm{lbl}} \& \operatorname{ncheck}(y, z)$

- label $(x, y) \stackrel{\text { def }}{\equiv}$ if $\mathrm{EQ}\left(x, \tau_{2}\left(\pi_{2}\left(\tau_{1}(y)\right)\right)\right)$ then $\pi_{1}\left(\tau_{1}(y)\right)$

$$
\begin{aligned}
& \text { else if } \operatorname{EQ}\left(x, \tau_{2}\left(\pi_{2}\left(\tau_{2}(y)\right)\right)\right) \text { then } \pi_{1}\left(\tau_{2}(y)\right) \\
& \text { else if } \operatorname{EQ}\left(x, \tau_{2}\left(\pi_{2}\left(\tau_{3}(y)\right)\right)\right) \text { then } \pi_{1}\left(\tau_{3}(y)\right) \\
& \text { else } \mathbf{0}
\end{aligned}
$$

Here isin $(x, y)$ checks that if $x$ occur in message $y$ which itself is a triple. bcheck $\left(_{-},,_{-}\right)$ models the voter's check that if his (her) ballot presents in the list of label-ballot pairs and ncheck $\left({ }_{-},{ }_{-}\right)$models the voter's check that if his (her) nonce presents inside the ballot. label $(x, y)$ is the finding the label of $x$ in the message $y$ which itself is a tuple of label-ballot pairs. 


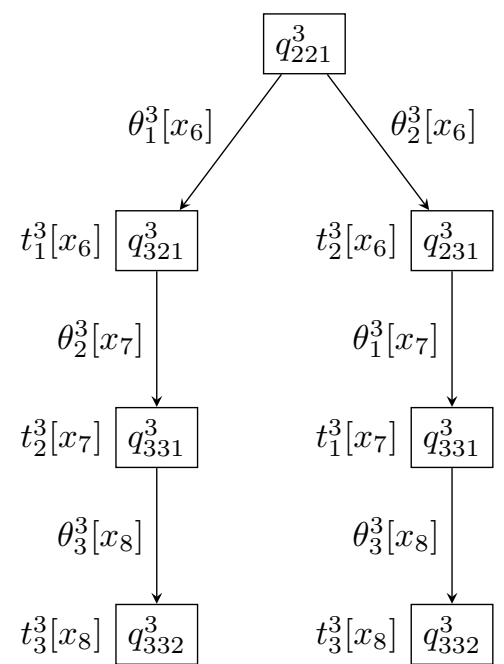

$$
\begin{aligned}
& \theta_{1}^{3}\left[x_{i}\right] \equiv \mathrm{EQ}\left(\operatorname{to}\left(x_{i}\right), A\right) \& \text { distbb } \\
& t_{1}^{3}\left[x_{i}\right] \equiv \text { if acc } \operatorname{ac}_{1} \& \text { bnlcheck }\left(c_{1}, n_{1}, x_{6}\right) \text { then } e_{1} \text { else } \mathbf{0} \\
& \theta_{2}^{3}\left[x_{i}\right] \equiv \mathrm{EQ}\left(\text { to }\left(x_{i}\right), B\right) \& \text { distbb } \\
& t_{2}^{3}\left[x_{i}\right] \equiv \text { if acc } \operatorname{ac}_{2} \& \text { bnlcheck }\left(c_{2}, n_{2}, x_{6}\right) \text { then } e_{2} \text { else } \mathbf{0} \\
& \theta_{3}^{3}\left[x_{8}\right] \equiv \mathrm{EQ}\left(\text { to }\left(x_{8}\right), M\right) \& \operatorname{dist}\left(D_{1}, D_{2}, D_{3}\right) \& \text { pchk } \\
& \&\left(\left(\operatorname{isin}_{\mathrm{k}_{1}} \& \operatorname{isin}_{\mathrm{k}_{2}}\right) \text { or }\left(\operatorname{not}\left(\operatorname{isin}_{\mathrm{k}_{1}} \text { or } \operatorname{isin}_{\mathrm{k}_{2}}\right)\right)\right) \\
& t_{3}^{3}\left[x_{8}\right] \equiv \operatorname{shufl}\left(P_{1}, P_{2}, P_{3}\right) \\
& \text { where distbb } \equiv \text { dist }_{1} \& \operatorname{dist}_{2} ; \operatorname{isin}_{\mathrm{k}_{k}} \equiv \mathrm{isin}\left(\mathrm{k}_{k}, D\right) \\
& e_{k} \equiv\left\{\left\langle\text { label }\left(c_{k}, x_{6}\right), \mathrm{k}_{k}, \mathrm{ph}_{3}\right\rangle\right\}_{\mathrm{pk}_{\mathrm{e}}\left(n_{M}\right)}^{\mathrm{r}\left(n^{k 2}\right)} \\
& \operatorname{acc}_{k} \equiv \operatorname{acc}\left(c_{k}, \mathrm{pk}, r_{1}, x_{3}\right) \text { or acc }\left(c_{k}, \mathrm{pk}, r_{1}, x_{4}\right) \\
& \operatorname{dist}_{k} \equiv \operatorname{dist}\left(\pi_{k}\left(\tau_{1}\left(x_{6}\right)\right), \pi_{k}\left(\tau_{2}\left(x_{6}\right)\right), \pi_{k}\left(\tau_{3}\left(x_{6}\right)\right)\right) \\
& P_{m} \equiv\left\langle\tau_{1}\left(D_{m}\right), \tau_{2}\left(D_{m}\right)\right\rangle, m \in\{1,2,3\} \\
& D_{j} \equiv \operatorname{dec}\left(\tau_{j}\left(x_{8}\right), \text { sk }_{\mathrm{e}}(m)\right), j \in\{1,2,3\} \\
& \mathrm{pk} \equiv \mathrm{PK}_{\mathrm{AD}} ; r_{k} \equiv \mathrm{r}_{\mathrm{b}}\left(n_{\mathrm{b}}^{k}\right) ; \mathrm{k}_{k} \equiv \mathrm{k}_{\mathrm{c}}\left(n_{\mathrm{c}}^{k}\right) \\
& D \equiv\left\langle\tau_{2}\left(D_{1}\right), \tau_{2}\left(D_{2}\right), \tau_{2}\left(D_{3}\right)\right\rangle ; i \text { is in }\{6,7\} \\
& \text { pchk } \equiv \mathrm{EQ}\left(\tau_{3}\left(D_{1}\right), \mathrm{ph}_{3}\right) \& \mathrm{EQ}\left(\tau_{3}\left(D_{2}\right), \mathrm{ph}_{3}\right) \& \\
& \mathrm{EQ}\left(\tau_{3}\left(D_{3}\right), \mathrm{ph}_{3}\right) ; k \text { is in }\{1,2\} \\
& c_{1}, c_{2} \text { are defined in Figure } 3.11
\end{aligned}
$$

Figure 3.13: Opening Phase of the FOO protocol

Finally, the Opening Phase is in Figure 3.13.

We obtain the execution terms from the transition system in the following way. For a given $b$ bit of the Challenger, $\Phi_{i}^{b}$ lists of terms representing what the attacker has seen up to step $i$ are created according to the rounds of the protocol. Notice that the initialization frame $\Phi_{0}$ is defined in Figure 3.11 and it is independent of the bit $b$. For the first step, the attacker's computation $f_{1}$ is applied to $\Phi_{0}$, and $f_{1}\left(\Phi_{0}\right)$ is sent to the Challenger, who then carries out the checks $\theta_{1}^{1}$ and $\theta_{2}^{1}$, and sends back

$$
\left.\phi_{1}^{b}:=\text { if } \theta_{1}^{1}\left[f_{1}\left(\Phi_{0}\right)\right] \text { then } t_{1}^{1}\left[f_{1}\left(\Phi_{0}\right)\right] \text { else (if } \theta_{2}^{1}\left[f_{1}\left(\Phi_{0}\right)\right] \text { then } t_{2}^{1}\left[f_{1}\left(\Phi_{0}\right)\right] \text { else } \mathbf{0}\right) \text {, }
$$

and we set $\Phi_{1}^{b} \stackrel{\text { def }}{\equiv} \Phi_{0}, \phi_{1}^{b}$. Similarly, in the second step, we have

$$
\begin{aligned}
& \text { if } \theta_{1}^{1}\left[f_{1}\left(\Phi_{0}\right)\right] \\
\phi_{2}^{b}:= & \text { then (if } \left.\theta_{2}^{1}\left[f_{2}\left(\Phi_{1}^{b}\right)\right] \text { then } t_{2}^{1}\left[f_{2}\left(\Phi_{1}^{b}\right)\right] \text { else } \mathbf{0}\right) \\
& \text { else (if } \left.\theta_{2}^{1}\left[f_{1}\left(\Phi_{0}\right)\right] \text { then (if } \theta_{1}^{1}\left[f_{2}\left(\Phi_{1}^{b}\right)\right] \text { then } t_{1}^{1}\left[f_{2}\left(\Phi_{1}^{b}\right)\right] \text { else } \mathbf{0}\right) \text { else } \mathbf{0} \text { ), }
\end{aligned}
$$

and $\Phi_{2}^{b} \stackrel{\text { def }}{\equiv} \Phi_{1}^{b}, \phi_{2}^{b}$, which is the end of the authentication phase. The other phases are done similarly continuing from $\Phi_{3}^{b}$. 
Let $\Phi_{m}^{b}$ be the last frame. In order to establish vote-privacy for the FOO protocol we have to show that the axioms and first-order inference rules imply that $\Phi_{m}^{0} \sim \Phi_{m}^{1}$. Then from the soundness theorem of [BCL14], it follows that there is no successful PPT attacker break the security game.

\subsection{Summary}

We recalled the computational primitives of FOO protocol from literature, including the commitments, blind digital signatures, and encryptions. We formalized the assumptions such as computational hiding and binding of the commitments, blindness property of the blind digital signatures, and the shuffling capability of the mixer using the CCSA framework. We illustrated the effectiveness of the $\mathrm{BC}$ technique for attack detection by finding the new attacks on vote privacy, a computational attack due to unequal vote lengths, a computational attack due to uneven blind signature lengths, a Dolev-Yao style replay attack, and a man-in-the-middle attack. We gave a game-based definition of vote privacy of the FOO protocol using the $\mathrm{BC}$ technique for indistinguishability. We presented a transition system for authentication, voting, and opening phases of the protocol. 


\section{Chapter 4}

\section{Proofs of Vote Privacy}

In this chapter, we present some auxiliary theorems, including the one in which we show how the combination of CompHid, CommEQL, UBNotUndefined, and Blindness allow us to fix the inadequacy of the blindness property. We present a proof of vote privacy (defined in 3.4.1) of the FOO voting protocol using the core axioms (see Table 2.1), the axioms (see Tables 3.7 and 3.8) of the cryptographic assumptions, and the other assumptions (see Table 3.1), in the provable security model using the $\mathrm{BC}$ technique. We show that the attacker can not detect feasibly when the honest voters switch their votes. This is true even if the protocol executions consist of all three phases authentication, voting, and opening.

\subsection{Auxiliary Theorems}

Inspired by the examples presented in [BCEO19], we present some properties of the \& symbol that can be proved using the core axioms and the theorem 2.4 .

Proposition 4.1 Assuming the core axioms and using theorem 2.4, we prove the following:

1. (Unfold \&) IfSAme, IfEval, IfTrue, IfFalse, EqRefl, EqCong $\vdash$ 
if $b_{1} \& b_{2}$ then $t_{1}$ else $t_{2}=$ if $b_{1}$ then (if $b_{2}$ then $t_{1}$ else $t_{2}$ ) else $t_{2}$.

2. (Swapping Branches) For any terms $b_{0}, b_{1}, t, t_{0}, t_{1}, t_{2}$, we have,

if $b_{0} \& b_{1}$ then $t$

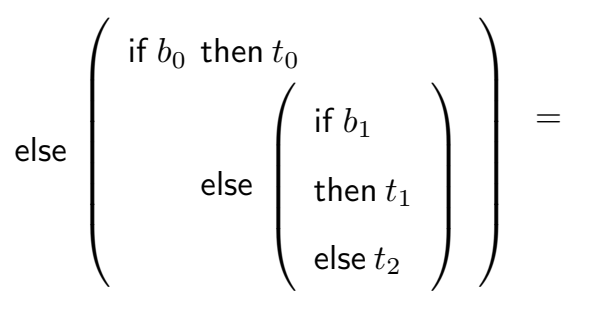

if $b_{0} \& b_{1}$ then $t$

$$
\text { else }\left(\begin{array}{l}
\text { if } b_{1} \text { then } t_{1} \\
\text { else } \left.\left(\begin{array}{l}
\text { if } b_{0} \\
\text { then } t_{0} \\
\text { else } t_{2}
\end{array}\right)\right)
\end{array}\right)
$$

3. ( СOMm) IfSAme, IFMorph, IFTF, IfEval $\vdash(x \& y)=(y \& x)$.

4. (ASSOC) COMm, IfSAme, IfEval, IfMorph, IfFalse $\vdash$

$$
x \&(y \& z)=(x \& y) \& z .
$$

5. IfSAME, EQREFL $\vdash(x \&$ false $)=$ false.

6. IFTF, EQREFL $\vdash(x \&$ true $)=x$.

7. IfEval, IfTrue, IfSAme $\vdash x \&(\operatorname{not}(x))=$ false.

8. IfMorph, IfTrue, IfFalse, IFTF $\vdash \operatorname{not}(\operatorname{not}(x))=x$.

Proof: We present the proofs one by one.

1.

LHS $\stackrel{\text { UNFOLD \& }}{=}$ if (if $b_{1}$ then $b_{2}$ else false ) then $t_{1}$ else $t_{2}$

$$
\begin{array}{r}
\stackrel{\text { IFSAME }}{=} \quad \text { if } b_{1} \text { then (if (if } b_{1} \text { then } b_{2} \text { else false) then } t_{1} \text { else } t_{2} \text { ) } \\
\text { else (if (if } b_{1} \text { then } b_{2} \text { else false) then } t_{1} \text { else } t_{2} \text { ) }
\end{array}
$$

IFEval, IFTrue, IFFALSE

if $b_{1}$ then (if $b_{2}$ then $t_{1}$ else $t_{2}$ ) else $t_{2}$. 
if $b_{0} \& b_{1}$ then $t$

2. LHS IFMORPH

$$
\text { else }\left(\begin{array}{c}
\text { if } \left.b_{1} \text { then (if } b_{0} \text { then } t_{0} \text { else } t_{1}\right) \\
\text { else (if } \left.b_{0} \text { then } t_{0} \text { else } t_{2}\right)
\end{array}\right)
$$

Using the fact that $\operatorname{not}\left(b_{0} \& b_{1}\right) \& b_{1}=\operatorname{not}\left(b_{0}\right)$, we get the required term on the right side.

3.

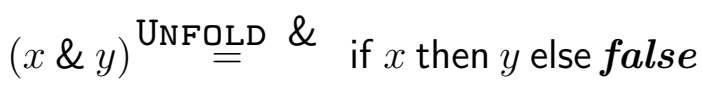

$$
\begin{aligned}
& \stackrel{\text { IFTF }}{=} \text { if } x \text { then (if } y \text { then true else false ) else false } \\
& \text { IFSAME } \text { if } y \text { then (if } x \text { then (if } y \text { then true else false ) else false ) } \\
& \text { else (if } x \text { then (if } y \text { then true else false ) else false ) } \\
& \text { IFEvAL if } y \text { then (if } x \text { then true else false) } \\
& \text { else (if } x \text { then false else false ) } \\
& \stackrel{\text { IFSAME }}{=} \text { if } y \text { then (if } x \text { then true else false ) else false } \\
& \stackrel{\mathrm{IFTF}}{=} \text { if } y \text { then } x \text { else false. }
\end{aligned}
$$

4 .

$$
\begin{gathered}
x \&(y \& z) \stackrel{\text { IFMORPH }}{=} \text { if } y \text { then (if } x \text { then } z \text { else false) } \\
\text { else (if } x \text { then false else false) } \\
(x \& y) \& z \stackrel{\text { IFSAME }}{=} \text { if } y \text { then (if } x \text { then } z \text { else false ) else false } \\
\text { IFMORPH } \\
\text { if } y \text { then (if } x \text { then } z \text { else false ) } \\
\text { else (if false then } z \text { else false) }
\end{gathered}
$$




$$
\stackrel{\mathrm{IFFALSE}_{\mathrm{A}}}{=} \text { if } y \text { then (if } x \text { then } z \text { else false ) else false. }
$$

5. $(x \&$ false $) \stackrel{\text { UNFOLD }}{=}$ if $x$ then false else false $\stackrel{\text { IFSAME }}{=}$ false.

6. $(x \&$ true $) \stackrel{\text { UNFOLD }}{=}$ if $x$ then true else false $\stackrel{\text { IFTF }}{=} x$.

7.

$$
\begin{gathered}
x \&(\operatorname{not}(x)) \stackrel{\text { UNFOLD }}{=} \text { if } x \text { then not }(x) \text { else false } \\
\text { UNFOLDnot } \text { if } x \text { then (if } x \text { then false else true ) else false } \\
\stackrel{\text { IFEVAL }}{=} \text { if } x \text { then false else false } \\
\text { IFSAME } \text { false. }
\end{gathered}
$$

8.

$$
\begin{gathered}
\operatorname{not}(\operatorname{not}(x)) \stackrel{\text { UNFOLD }}{=} \text { if (if } x \text { then false else true ) then false else true } \\
\text { IFMORPH } \\
\text { if } x \text { then (if false then false else true ) } \\
\text { IFTRUE, IFFALSE } \text { if } x \text { then false else true } \stackrel{\mathrm{IFTF}_{=}}{=} .
\end{gathered}
$$

Here we present some extended versions of the axioms FRESHIND, FunCAPP, and PAIREQL, which are provable using core axioms and significantly reduce the number of steps in the proofs of vote privacy. We realized the importance of these results while mechanizing the proofs in Coq.

\section{Proposition 4.2 (Extending FreshInd)}

Let $t[x]$ be a term that contains a variable $x$. Let $\vec{z}$ be a list of ground terms and $n_{1}$ and $n_{2}$ be names such that fresh $\left(n_{1}, n_{2} ; \vec{z}, t\right)$ holds. 


$$
\vec{z}, t\left[n_{1}\right] \sim \vec{z}, t\left[n_{2}\right]
$$

Proof: It is not hard to see the formula is derivable using FreshInd and FunCAPP.

We extend the FuncAPp in the proposition below.

Proposition 4.3 (Extending FuncApp) For any function $f \in \mathcal{F} \cup \mathcal{G}$,

$$
\begin{gathered}
\vec{z}, b \text {, if } b \text { then } x \text { else } y \sim \vec{z}^{\prime}, b^{\prime} \text {, if } b^{\prime} \text { then } x^{\prime} \text { else } y^{\prime} \longrightarrow \\
\vec{z}, b, \text { if } b \text { then } f(\vec{z}, b, x) \text { else } t \sim \vec{z}^{\prime}, b^{\prime} \text {, if } b^{\prime} \text { then } f\left(\vec{z}^{\prime}, b^{\prime}, x^{\prime}\right) \text { else } t^{\prime}
\end{gathered}
$$

This is because:

by FUNCAPP,

$$
\vec{z}, b, \quad \begin{aligned}
& \text { if } b \text { then } x \\
& \text { else } y
\end{aligned}, f\left(\vec{z}, b, \quad \begin{array}{l}
\text { if } b \text { then } x \\
\text { else } y
\end{array}\right) \sim \vec{z}^{\prime}, b^{\prime}, \quad \begin{array}{r}
\text { if } b^{\prime} \text { then } x^{\prime} \\
\text { else } y^{\prime}
\end{array}, f\left(\vec{z}^{\prime}, b^{\prime}, \quad \begin{array}{r}
\text { if } b^{\prime} \text { then } x^{\prime} \\
\text { else } y^{\prime}
\end{array}\right)
$$

Using IFMoRPH and RESTR, we have

$$
\vec{z}, b \text {, if } b \text { then } f(\vec{z}, b, x) \text { else } f(\vec{z}, b, y) \sim \vec{z}^{\prime}, b^{\prime} \text {, if } b^{\prime} \text { then } f\left(\vec{z}^{\prime}, b^{\prime}, x^{\prime}\right) \text { else } f\left(\vec{z}^{\prime}, b^{\prime}, y^{\prime}\right)
$$

Again using FuncApP, we get

$$
\vec{z}, b, \quad \text { if } b \text { then }\left(\begin{array}{c}
\text { if } b \text { then } f(\vec{z}, b, x) \\
\text { else } f(\vec{z}, b, y)
\end{array}\right) \sim \vec{z}^{\prime}, b^{\prime}, \quad \text { if } b^{\prime} \text { then }\left(\begin{array}{c}
\text { if } b^{\prime} \text { then } f\left(\vec{z}^{\prime}, b^{\prime}, x^{\prime}\right) \\
\text { else } f\left(\vec{z}^{\prime}, b^{\prime}, y^{\prime}\right)
\end{array}\right)
$$

Then using IfEval, IfTrue, IfFalse, we have

$$
\vec{z}, b \text {, if } b \text { then } f(\vec{z}, b, x) \text { else } t \sim \vec{z}^{\prime}, b^{\prime} \text {, if } b^{\prime} \text { then } f\left(\vec{z}^{\prime}, b^{\prime}, x^{\prime}\right) \text { else } t^{\prime}
$$


Proposition 4.4 (Extending PairEQL)

if $\operatorname{EQL}\left(x_{1}, y_{1}\right)$ then $\left(\begin{array}{l}\text { if } b \text { then }\left(\begin{array}{l}\text { if } \operatorname{EQL}\left(x_{2}, y_{2}\right) \\ \text { then } \operatorname{EQL}\left(\left\langle x_{1}, x_{2}\right\rangle,\left\langle y_{1}, y_{2}\right\rangle\right) \\ \text { else } \boldsymbol{t r u e}\end{array}\right) \\ \text { else } \text { true }\end{array}\right)=$ true
else true

Proof: Rewriting the LHS as the following:

$$
\text { if } \left.\operatorname{EQL}\left(x_{1}, y_{1}\right) \text { then }\left(\begin{array}{l}
\text { if } \operatorname{EQL}\left(x_{2}, y_{2}\right) \\
\text { then }\left(\begin{array}{l}
\text { if } \operatorname{EQL}\left(x_{1}, y_{1}\right) \text { \& } \operatorname{EQL}\left(x_{2}, y_{2}\right) \\
\text { then } \operatorname{EQL}\left(\left\langle x_{1}, x_{2}\right\rangle,\left\langle y_{1}, y_{2}\right\rangle\right) \\
\text { else true }
\end{array}\right) \\
\text { else true true true }
\end{array}\right)\right)
$$

Then rewriting PAIREQL and repeatedly rewriting IFSAME completes the proof.

In the proposition below, we show that if two votes are valid, then they should have the same length.

Proposition 4.5 (Equal Vote lengths) IfMorph, IfEval, IfSame, IfFalse, Lemma 10, EQREFL $\vdash$ if vcheck $\left[v_{b}\right]$ \& vcheck $\left[v_{1-b}\right]$ then $\operatorname{EQL}\left(v_{b}, v_{1-b}\right)$ else true $=$ true where vcheck $[v] \equiv \mathrm{EQ}\left(v, C_{1}\right)$ or $\mathrm{EQ}\left(v, C_{2}\right)$ or $\mathrm{EQ}\left(v, C_{3}\right), v_{b} \equiv \mathrm{V}_{b}\left(x_{1}\right)$ and $v_{1-b} \equiv \mathrm{V}_{1-b}\left(x_{1}\right)$ First, we define the following notation: $\mathrm{S} \equiv$ IfSAme, IfEval, IfTrue, IFFaLse, 


$$
\begin{aligned}
& t \equiv \text { if }\left(\mathrm{EQ}\left(v_{b}, C_{2}\right) \text { or } \mathrm{EQ}\left(v_{b}, C_{3}\right)\right) \&\left(\mathrm{EQ}\left(v_{1-b}, C_{2}\right) \text { or } \operatorname{EQ}\left(v_{1-b}, C_{3}\right)\right) \text { then } \operatorname{EQL}\left(v_{b}, v_{1-b}\right) \\
& \text { else true } \\
& t^{\prime} \equiv \text { if }\left(\mathrm{EQ}\left(v_{b}, C_{3}\right) \text { or } \operatorname{EQ}\left(v_{1-b}, C_{3}\right)\right) \text { then } \operatorname{EQL}\left(v_{b}, v_{1-b}\right) \text { else } \boldsymbol{t r u e}
\end{aligned}
$$

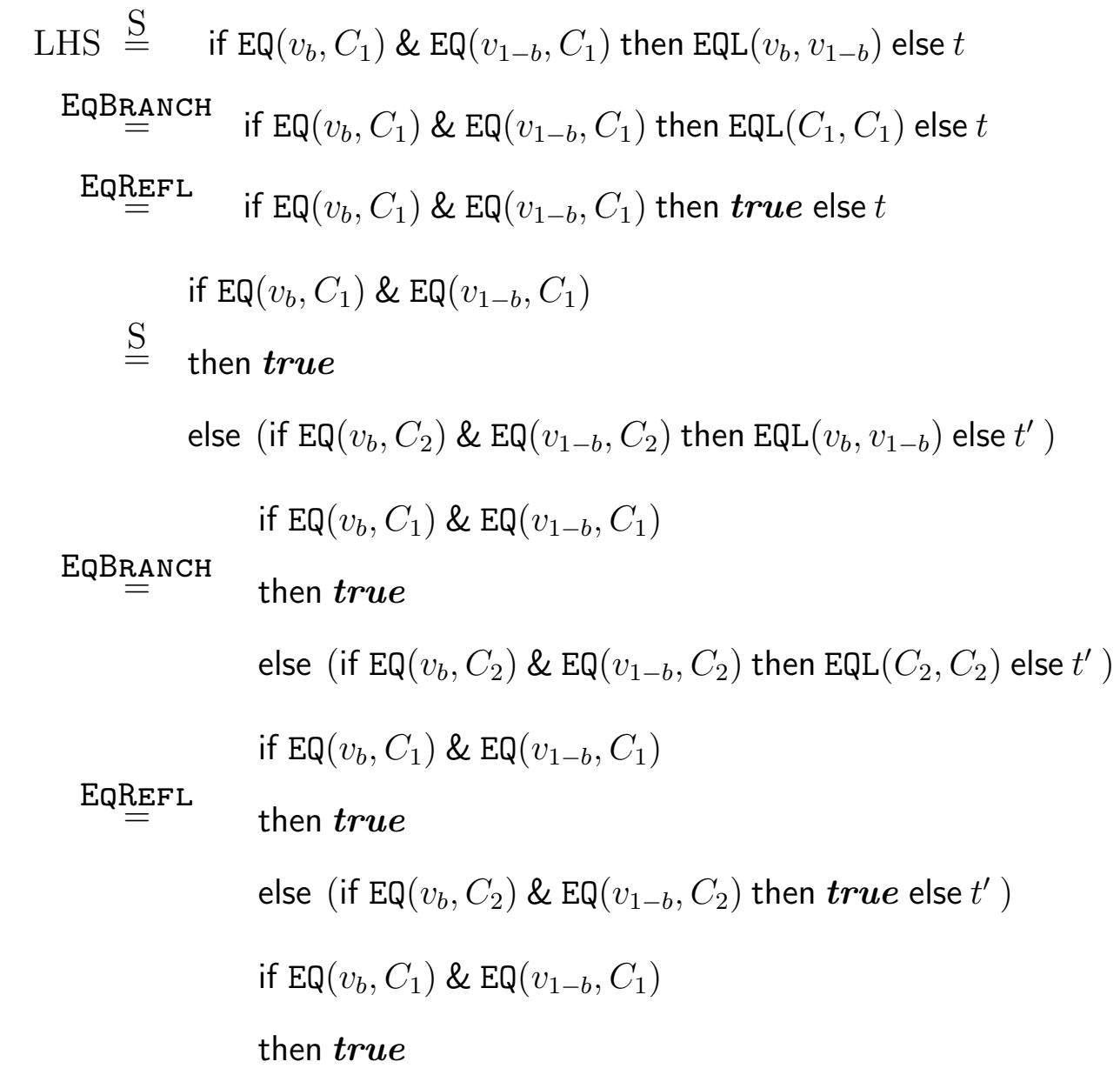

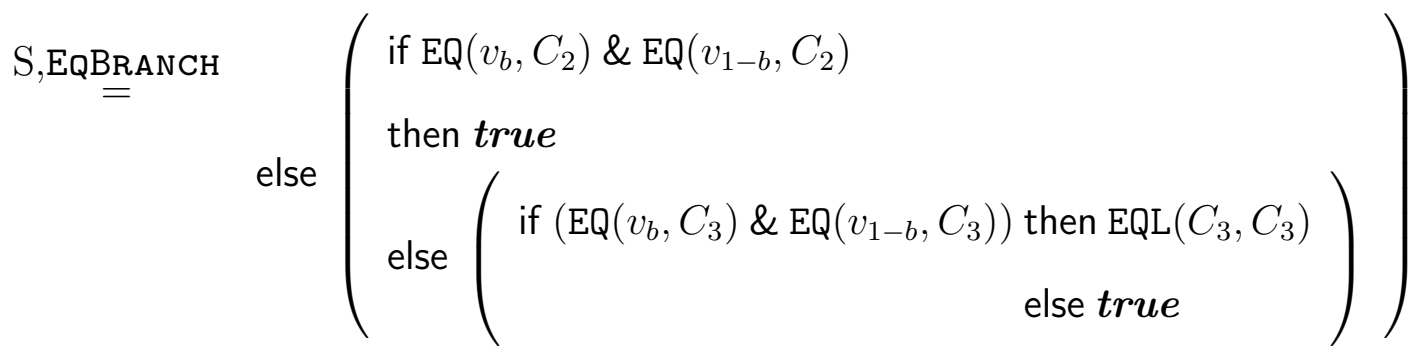

EQREFL, EQBRANCH true. 
We extend the CompHid axiom using FuncApP and other core axioms such as IfMorph, REstr, IfEval, IfTrue, and IfFalse below.

\section{Proposition 4.6 (Extending CompHid)}

Let $t, t_{1}$, and $t_{2}$ be three ground terms. Let $t_{0}[x, y]$ be a term that has two variables $x$ and $y$. Let $\vec{z}$ be a list of ground terms and $n_{1}$ and $n_{2}$ be names such that $\operatorname{fresh}\left(n_{1}, n_{2} ; \vec{t}, t_{0}, t_{1}, t_{2}\right)$ holds.

$$
\begin{aligned}
& \text { if } \operatorname{EQL}\left(t_{1}, t_{2}\right) \quad \text { if } \operatorname{EQL}\left(t_{1}, t_{2}\right) \\
& \vec{z}, \text { then } t_{0}\left[\operatorname{com}\left(t_{1}, \mathrm{k}_{\mathrm{c}}\left(n_{1}\right)\right), \operatorname{com}\left(t_{2}, \mathrm{k}_{\mathrm{c}}\left(n_{2}\right)\right)\right] \sim \vec{z}, \text { then } t_{0}\left[\operatorname{com}\left(t_{2}, \mathrm{k}_{\mathrm{c}}\left(n_{1}\right)\right), \operatorname{com}\left(t_{1}, \mathrm{k}_{\mathrm{c}}\left(n_{2}\right)\right)\right] \\
& \text { else } t \quad \text { else } t
\end{aligned}
$$

Proof: Recall the CompHid axiom as the following:

$$
\begin{array}{ll}
\text { if } \operatorname{EQL}\left(t_{1}, t_{2}\right) & \text { if } \operatorname{EQL}\left(t_{1}, t_{2}\right) \\
\vec{z}, \text { then }\left\langle\operatorname{com}\left(t_{1}, \mathrm{k}_{\mathrm{c}}\left(n_{1}\right)\right), \operatorname{com}\left(t_{2}, \mathrm{k}_{\mathrm{c}}\left(n_{2}\right)\right)\right\rangle \sim \vec{z}, & \text { then }\left\langle\operatorname{com}\left(t_{2}, \mathrm{k}_{\mathrm{c}}\left(n_{1}\right)\right), \operatorname{com}\left(t_{1}, \mathrm{k}_{\mathrm{c}}\left(n_{2}\right)\right)\right\rangle \\
\text { else } t & \text { else } t
\end{array}
$$

Using FunCAPP, IFMORPH, RESTR,

$$
\begin{aligned}
& \text { if } \operatorname{EQL}\left(t_{1}, t_{2}\right) \quad \text { if } \operatorname{EQL}\left(t_{1}, t_{2}\right) \\
& \vec{z}, \text { then } t_{0}\left[\operatorname{com}\left(t_{1}, \mathrm{k}_{\mathrm{c}}\left(n_{1}\right)\right), \operatorname{com}\left(t_{2}, \mathrm{k}_{\mathrm{c}}\left(n_{2}\right)\right)\right] \sim \vec{z}, \text { then } t_{0}\left[\operatorname{com}\left(t_{2}, \mathrm{k}_{\mathrm{c}}\left(n_{1}\right)\right), \operatorname{com}\left(t_{1}, \mathrm{k}_{\mathrm{c}}\left(n_{2}\right)\right)\right] \\
& \text { else } t_{0}\left[\pi_{1}(t), \pi_{2}(t)\right] \quad \text { else } t_{0}\left[\pi_{1}(t), \pi_{2}(t)\right]
\end{aligned}
$$

Again using FunCAPP,

$$
\begin{aligned}
& \vec{z}, \text { if } \operatorname{EQL}\left(t_{1}, t_{2}\right) \text { then }\left(\begin{array}{c}
\text { if } \operatorname{EQL}\left(t_{1}, t_{2}\right) \text { then } t_{0}\left[\operatorname{com}\left(t_{1}, \mathrm{k}_{\mathrm{c}}\left(n_{1}\right)\right), \operatorname{com}\left(t_{2}, \mathrm{k}_{\mathrm{c}}\left(n_{2}\right)\right)\right] \\
\text { else } t_{0}\left[\pi_{1}(t), \pi_{2}(t)\right]
\end{array}\right) \text { else } t \\
& \sim \vec{z}, \text { if } \operatorname{EQL}\left(t_{1}, t_{2}\right) \text { then }\left(\begin{array}{c}
\text { if } \operatorname{EQL}\left(t_{1}, t_{2}\right) \text { then } t_{0}\left[\operatorname{com}\left(t_{2}, \mathrm{k}_{\mathrm{c}}\left(n_{1}\right)\right), \operatorname{com}\left(t_{1}, \mathrm{k}_{\mathrm{c}}\left(n_{2}\right)\right)\right] \\
\text { else } t_{0}\left[\pi_{1}(t), \pi_{2}(t)\right]
\end{array}\right) \text { else } t
\end{aligned}
$$

It is easy to see the proof from IfEval, IfTrue, and IfFalse. 


\subsubsection{Notations and Conventions}

Let $v_{0}, v_{1}, t$ be ground terms, let $\vec{z}$ be a list of ground terms. Assume $\operatorname{EQL}\left(v_{0}, v_{1}\right)=$

true. Let $t_{0}[x, y]$ and $t_{1}[x, y]$ be terms containing two variables $x$ and $y$. Let $n_{\mathrm{b} 0}, n_{\mathrm{b} 1}$, $n_{\mathrm{c} 0}, n_{\mathrm{c} 1}, n_{0}$ and $n_{1}$ be names such that fresh $\left(n_{0}, n_{1}, n_{\mathrm{b} 0}, n_{\mathrm{b} 1}, n_{\mathrm{c} 0}, n_{\mathrm{c} 1} ; \vec{z}, t, v_{0}, v_{1}, t_{0}, t_{1}\right)$ holds.

For readability, we introduce the following notations:

\section{Notation}

$t_{i}^{\mathrm{j}} \equiv t_{i}\left[\mathrm{~b}\left(c_{0}^{j}\left[v_{j}\right], t, \mathrm{r}_{\mathrm{b}}^{0}\right), \mathrm{b}\left(c_{1}^{j}\left[v_{1-j}\right], t, \mathrm{r}_{\mathrm{b}}^{1}\right)\right]$

$\mathrm{k}_{\mathrm{c}}^{i} \equiv \mathrm{k}_{\mathrm{c}}\left(n_{\mathrm{ci}}\right) ; \mathrm{r}_{\mathrm{b}}^{i} \equiv \mathrm{r}_{\mathrm{b}}\left(n_{\mathrm{bi}}\right)$

$c_{i}^{j}\left[v_{j \oplus i}\right] \equiv \operatorname{com}\left(v_{j \oplus i}, \mathrm{k}_{\mathrm{c}}^{i}\right)$

$b_{i}^{j}\left[v_{j \oplus i}\right] \equiv \mathrm{b}\left(c_{i}^{j}\left[v_{j \oplus i}\right], t, \mathrm{r}_{\mathrm{b}}^{i}\right)$

$\operatorname{acc}_{i}^{j} \equiv \operatorname{acc}\left(c_{i}^{j}\left[v_{j \oplus i}\right], t, \mathrm{r}_{\mathrm{b}}^{i}, t_{i}^{j}\right)$

$\mathrm{ub}_{i}^{j}\left[v_{j \oplus i}\right] \equiv \mathrm{ub}\left(c_{i}^{j}\left[v_{j \oplus i}\right], t, \mathrm{r}_{\mathrm{b}}^{i}, t_{i}^{j}\right)$

$e_{i}^{j}\left[v_{j \oplus i}\right] \equiv\left\{\left\langle\left\langle c_{i}^{j}\left[v_{j \oplus i}\right], \mathrm{ub}_{i}^{j}\left[v_{j \oplus i}\right], n_{i}\right\rangle, \mathrm{ph}_{2}\right\rangle\right\}_{\mathrm{pk}_{\mathrm{e}}\left(n_{M}\right)}^{\mathrm{r}\left(n_{\mathrm{e}}\right)}$

\section{Term}

Context term with a substitution Commitment and blinding keys

Commitments

Blinded commitments

Acceptance check

Unblinded term

Encryptions

Unless otherwise stated, the superscript $j$ is used to differentiate left side terms from the right side terms. In particular, if $j$ is equal to 0 , then the term belongs to the left side, and it represents the right side term when the $j$ value is 1 . Similarly, $i$ simulates the moves of the two honest voters in the protocol. If $i$ is equal to 0 , then the term belongs to the first voter whereas the second voter's term represented using the value 1.

We also introduce a notation $u_{i}^{j}\left[v_{j \oplus i}\right] \equiv\left\langle\mathrm{ub}_{i}^{j}\left[v_{j \oplus i}\right], c_{i}^{j}\left[v_{j \oplus i}\right], \mathrm{k}_{\mathrm{c}}^{i}\right\rangle$ to represent the triple which includes the unblinded term $\mathrm{ub}_{i}^{j}\left[v_{j \oplus i}\right]$, the commitment $c_{i}^{j}\left[v_{j \oplus i}\right]$, and the commitment key $k_{\mathrm{c}}^{i}$. 
To represent a term that model the situation where both the blind signatures, only one of the blind signatures, or none of them accepted, we introduce a notation $t^{j}$. As usual, $t^{0}$ represents the left term and $t^{1}$ represents the right term. The terms are defined below.

$$
\begin{array}{r}
t^{0} \equiv \text { if } \operatorname{acc}_{0}^{0} \& \operatorname{acc}_{1}^{0} \text { then }\left\langle\left\langle\mathrm{ub}_{0}^{0}\left[v_{0}\right], c_{0}^{0}\left[v_{0}\right], \mathrm{k}_{\mathrm{c}}^{0}\right\rangle,\left\langle\mathrm{ub}_{1}^{0}\left[v_{1}\right], c_{1}^{0}\left[v_{1}\right], \mathrm{k}_{\mathrm{c}}^{1}\right\rangle\right\rangle \\
\text { else if } \operatorname{acc}_{0}^{0} \text { then }\left\langle\left\langle\mathrm{ub} \mathrm{b}_{0}^{0}\left[v_{0}\right], c_{0}^{0}\left[v_{0}\right]\right\rangle, \perp\right\rangle \\
\text { else if } \operatorname{acc}_{1}^{0} \text { then }\left\langle\perp,\left\langle\mathrm{ub}_{1}^{0}\left[v_{1}\right], c_{1}^{0}\left[v_{1}\right]\right\rangle\right\rangle \\
\text { else }\langle\perp, \perp\rangle
\end{array}
$$

We also introduce a few notations to represent the attacker knowledge during the protocol.

$$
\begin{array}{rlrl}
\Phi_{i} & \equiv z, b_{0}^{i}\left[v_{i}\right], b_{1}^{i}\left[v_{1-i}\right] & \Phi_{1}^{j} \equiv \Phi_{j}, e_{0}^{j}\left[v_{j}\right] \\
\Phi_{2}^{j} \equiv \Phi_{1}^{j}, e_{1}^{j}\left[v_{1-j}\right] & \Phi_{3}^{j} \equiv \Phi_{2}^{j}, d_{v}\left(v_{1}^{j}, v_{2}^{j}, v_{3}^{j}\right)[x] \\
\Phi_{4}^{j} \equiv \Phi_{3}^{j}, l_{0}^{j}\left[v_{j}\right] & \Phi_{5}^{j} \equiv \Phi_{4}^{j}, l_{1}^{j}\left[v_{1-j}\right]
\end{array}
$$

where $d_{v}\left(v_{1}^{j}, v_{2}^{j}, v_{3}^{j}\right)[x]$ represents the mixer's term of the voting phase which is defined as

$$
d_{v}\left(v_{1}^{j}, v_{2}^{j}, v_{3}^{j}\right)[x] \equiv \text { if } \operatorname{dist}\left(v_{1}^{j}, v_{2}^{j}, v_{3}^{j}\right) \& \operatorname{pchk}_{v}\left(v_{1}^{j}, v_{2}^{j}, v_{3}^{j}\right) \text { then } x \text { else } \perp
$$

Of course, $v_{i}^{j} \equiv \operatorname{dec}\left(\tau_{i}\left(f_{2}\left(\Phi_{2}^{j}\right)\right)\right.$, sk $\left.{ }_{\mathrm{e}}\left(n_{M}\right)\right), i=1,2,3$, represents the decrypted messages of the mixer. $\operatorname{dist}\left(v_{1}^{j}, v_{2}^{j}, v_{3}^{j}\right)$ checks if the decrypted messages are distinct (see 3.5.4 for abbreviations). $\operatorname{pchk}_{v}\left(v_{1}^{j}, v_{2}^{j}, v_{3}^{j}\right)$ checks that if the decrypted messages belong to the voting phase and is defined as $\operatorname{pchk}_{v}\left(v_{1}^{j}, v_{2}^{j}, v_{3}^{j}\right) \equiv \mathrm{EQ}\left(\pi_{2}\left(v_{1}^{j}\right), \operatorname{ph}_{2}\right) \& \mathrm{EQ}\left(\pi_{2}\left(v_{2}^{j}\right), \operatorname{ph}_{2}\right) \&$ $\operatorname{EQ}\left(\pi_{2}\left(v_{3}^{j}\right), \mathrm{ph}_{2}\right)$.

We also introduce a notation $l_{i}^{j}\left[v_{j \oplus i}\right]$ that represents the message of the honest 
voter in the opening phase and is defined as

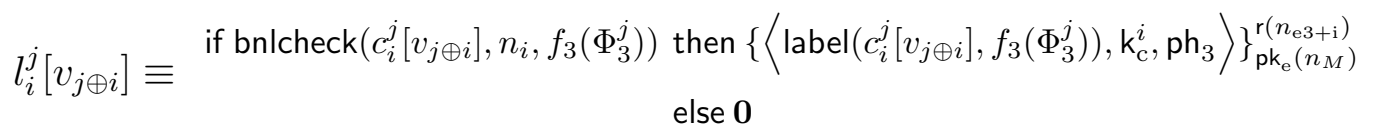

where the condition bnlcheck $\left(c_{i}^{j}\left[v_{j \oplus i}\right], n_{i}, f_{3}\left(\Phi_{3}^{j}\right)\right)$ checks that if the commitment $c_{i}^{j}\left[v_{j \oplus i}\right]$ and the name $n_{i}$ appear on the Bulletin Board (BB) and the the length of its label is equal to the constant $\ell_{l b l}$ and is defined in 3.5.4.

Finally, we introduce a notation for the mixer term of the opening phase. As usual, mixer waits to arrive the encrypted messages from the attacker and decrypts them, and checks that if the received messages are distinct, the messages belong to the voting phase, and checks if either both the commitment keys $k_{c}^{0}$ and $k_{c}^{1}$ appear in the decrypted messages or none of them do. If all these checks succeed, the mixer then outputs the decrypted messages in the lexicographic order to the attacker. Accordingly, we introduce the following notation:

$$
\begin{aligned}
& \text { if } \operatorname{dist}\left(o_{1}^{j}, o_{2}^{j}, o_{3}^{j}\right) \& \operatorname{pchk}_{o}\left(o_{1}^{j}, o_{2}^{j}, o_{3}^{j}\right) \&\left(\left(\operatorname{isin}_{\mathbf{k}_{\mathrm{c}}^{0}} \& \operatorname{isin}_{\mathbf{k}_{\mathrm{c}}^{1}}\right) \text { or }\left(\operatorname{not}\left(\operatorname{isin}_{\mathbf{k}_{\mathrm{c}}^{0}} \operatorname{or}_{\operatorname{isin}_{\mathbf{k}_{\mathrm{c}}^{1}}}\right)\right)\right) \\
d_{o}\left(o_{1}^{j}, o_{2}^{j}, o_{3}^{j}\right) \equiv & \text { then } \operatorname{shufl}\left(p_{1}\left(o_{1}^{j}\right), p_{2}\left(o_{2}^{j}\right), p_{3}\left(o_{3}^{j}\right)\right) \\
& \text { else } \perp
\end{aligned}
$$

where $o_{i}^{j} \equiv \operatorname{dec}\left(\tau_{i}\left(f_{5}\left(\Phi_{5}^{j}\right)\right)\right.$, sk $\left.{ }_{\mathrm{e}}\left(n_{M}\right)\right)$ represents the mixer's decryptions, the condition $\operatorname{dist}\left(o_{1}^{j}, o_{2}^{j}, o_{3}^{j}\right)$ checks that if the received messages are distinct, $\operatorname{isin}_{\mathbf{k}_{\mathrm{c}}^{i}} \equiv \operatorname{isin}\left(\mathrm{k}_{\mathrm{c}}^{i},\left\langle o_{1}^{j}, o_{2}^{j}, o_{3}^{j}\right\rangle\right)$ checks if the commitment key $\mathrm{k}_{\mathrm{c}}^{i}$ appear in the decrypted messages, and $\operatorname{pchk}_{o}\left(o_{1}^{j}, o_{2}^{j}, o_{3}^{j}\right)$ make sure that the messages belong to the opening phase which is defined as

$$
\operatorname{pchk}_{o}\left(o_{1}^{j}, o_{2}^{j}, o_{3}^{j}\right) \equiv \operatorname{EQ}\left(\tau_{3}\left(o_{1}^{j}\right), \operatorname{ph}_{3}\right) \& \operatorname{EQ}\left(\tau_{3}\left(o_{2}^{j}\right), \operatorname{ph}_{3}\right) \& \mathrm{EQ}\left(\tau_{3}\left(o_{3}^{j}\right), \operatorname{ph}_{3}\right)
$$

Of course, $p_{i}(x) \equiv\left\langle\tau_{1}(x), \tau_{2}(x)\right\rangle$ represents the decrypted message without the phase number. 


\subsubsection{Extending Blindness axiom}

We extend the BLINDNESS axiom using the UBNotUnDEFINED and other core axioms in the Proposition below. Note that we also make the acceptance checks available to the attacker.

\section{Proposition 4.7 (Including acceptance checks)}

$$
\begin{gathered}
\vec{z}, b_{0}^{0}\left[v_{0}\right], b_{1}^{0}\left[v_{1}\right], \operatorname{acc}_{0}^{0} \& \operatorname{acc}_{1}^{0}, \text { if } \operatorname{acc}_{0}^{0} \& \operatorname{acc}_{1}^{0} \text { then }\left\langle u_{0}^{0}\left[v_{0}\right], u_{1}^{0}\left[v_{1}\right]\right\rangle \text { else }\langle\perp, \perp\rangle \\
\sim \vec{z}, b_{0}^{1}\left[v_{1}\right], b_{1}^{1}\left[v_{0}\right], \operatorname{acc}_{0}^{1} \& \operatorname{acc}_{1}^{1}, \text { if } \operatorname{acc}_{0}^{1} \& \operatorname{acc}_{1}^{1} \text { then }\left\langle u_{1}^{1}\left[v_{0}\right], u_{0}^{1}\left[v_{1}\right]\right\rangle \text { else }\langle\perp, \perp\rangle
\end{gathered}
$$

Proof: Assume the Blindness axiom:

$$
\begin{aligned}
& \vec{z}, b_{0}^{0}\left[v_{0}\right], b_{1}^{0}\left[v_{1}\right], \text { if } \operatorname{acc}_{0}^{0} \& \operatorname{acc}_{1}^{0} \text { then }\left\langle\mathrm{ub}_{0}^{0}\left[v_{0}\right], \mathrm{ub}_{1}^{0}\left[v_{1}\right]\right\rangle \text { else }\langle\perp, \perp\rangle \\
& \text { if } \operatorname{acc}\left(c_{1}^{0}\left[v_{1}\right], t, \mathrm{r}_{\mathrm{b}}^{0}, t_{0}^{1}\right) \& \operatorname{acc}\left(c_{0}^{0}\left[v_{0}\right], t, \mathrm{r}_{\mathrm{b}}^{1}, t_{1}^{1}\right) \\
& \sim \vec{z}, \mathrm{~b}\left(c_{1}^{0}\left[v_{1}\right], t, \mathrm{r}_{\mathrm{b}}^{0}\right), \mathrm{b}\left(c_{0}^{0}\left[v_{0}\right], t, \mathrm{r}_{\mathrm{b}}^{1}\right), \operatorname{then}\left\langle\mathrm{ub}\left(c_{0}^{0}\left[v_{0}\right], t, \mathrm{r}_{\mathrm{b}}^{1}, t_{1}^{1}\right), \mathrm{ub}\left(c_{1}^{0}\left[v_{1}\right], t, \mathrm{r}_{\mathrm{b}}^{0}, t_{0}^{1}\right)\right\rangle \\
& \text { else }\langle\perp, \perp\rangle
\end{aligned}
$$

Applying FuncAPP for projection $\pi_{1}$ on the last term and using IFMorPH, we have

$$
\begin{aligned}
& \vec{z}, b_{0}^{0}\left[v_{0}\right], b_{1}^{0}\left[v_{1}\right], \quad \begin{array}{c}
\text { if } \operatorname{acc}_{0}^{0} \& \operatorname{acc}_{1}^{0} \text { then }\left\langle\mathrm{ub}_{0}^{0}\left[v_{0}\right], \mathrm{ub}_{1}^{0}\left[v_{1}\right]\right\rangle \\
\text { else }\langle\perp, \perp\rangle
\end{array}, \quad \begin{array}{c}
\text { if } \operatorname{acc}_{0}^{0} \& \operatorname{acc}_{1}^{0} \text { then } \mathrm{ub}_{0}^{0}\left[v_{0}\right] \\
\text { else } \perp
\end{array} \\
& \text { if } \operatorname{acc}\left(c_{1}^{0}\left[v_{1}\right], t, \mathrm{r}_{\mathrm{b}}^{0}, t_{0}^{1}\right) \& \operatorname{acc}\left(c_{0}^{0}\left[v_{0}\right], t, \mathrm{r}_{\mathrm{b}}^{1}, t_{1}^{1}\right) \\
& \sim \vec{z}, \mathrm{~b}\left(c_{1}^{0}\left[v_{1}\right], t, \mathrm{r}_{\mathrm{b}}^{0}\right), \mathrm{b}\left(c_{0}^{0}\left[v_{0}\right], t, \mathrm{r}_{\mathrm{b}}^{1}\right), \text { then }\left\langle\mathrm{ub}\left(c_{0}^{0}\left[v_{0}\right], t, \mathrm{r}_{\mathrm{b}}^{1}, t_{1}^{1}\right), \mathrm{ub}\left(c_{1}^{0}\left[v_{1}\right], t, \mathrm{r}_{\mathrm{b}}^{0}, t_{0}^{1}\right)\right\rangle \text {, } \\
& \text { else }\langle\perp, \perp\rangle \\
& \text { if } \operatorname{acc}\left(c_{1}^{0}\left[v_{1}\right], t, \mathrm{r}_{\mathrm{b}}^{0}, t_{0}^{1}\right) \& \operatorname{acc}\left(c_{0}^{0}\left[v_{0}\right], t, \mathrm{r}_{\mathrm{b}}^{1}, t_{1}^{1}\right) \text { then } \mathrm{ub}\left(c_{0}^{0}\left[v_{0}\right], t, \mathrm{r}_{\mathrm{b}}^{1}, t_{1}^{1}\right) \text { else } \perp
\end{aligned}
$$

Again applying FunCAPP for appending $\perp$ and for EQ with arguments being the last 
term and the $\perp$, and using IFMorPh, RESTR we get

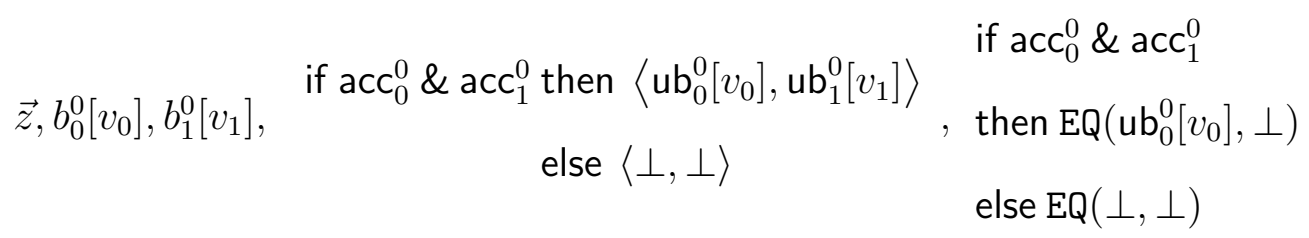

$$
\begin{aligned}
& \text { if } \operatorname{acc}\left(c_{1}^{0}\left[v_{1}\right], t, r_{\mathrm{b}}^{0}, t_{0}^{1}\right) \& \operatorname{acc}\left(c_{0}^{0}\left[v_{0}\right], t, r_{\mathrm{b}}^{1}, t_{1}^{1}\right) \\
& \sim \vec{z}, \mathrm{~b}\left(c_{1}^{0}\left[v_{1}\right], t, \mathrm{r}_{\mathrm{b}}^{0}\right), \mathrm{b}\left(c_{0}^{0}\left[v_{0}\right], t, \mathrm{r}_{\mathrm{b}}^{1}\right), \text { then }\left\langle\mathrm{ub}\left(c_{0}^{0}\left[v_{0}\right], t, \mathrm{r}_{\mathrm{b}}^{1}, t_{1}^{1}\right), \mathrm{ub}\left(c_{1}^{0}\left[v_{1}\right], t, \mathrm{r}_{\mathrm{b}}^{0}, t_{0}^{1}\right)\right\rangle \\
& \text { else }\langle\perp, \perp\rangle
\end{aligned}
$$

if $\operatorname{acc}\left(c_{1}^{0}\left[v_{1}\right], t, r_{\mathrm{b}}^{0}, t_{0}^{1}\right) \& \operatorname{acc}\left(c_{0}^{0}\left[v_{0}\right], t, r_{\mathrm{b}}^{1}, t_{1}^{1}\right)$ then $\mathrm{EQ}\left(\mathrm{ub}\left(c_{0}^{0}\left[v_{0}\right], t, r_{\mathrm{b}}^{1}, t_{1}^{1}\right), \perp\right)$ else $\mathrm{EQ}(\perp, \perp)$

Using UBNotUndEF INED, EQREFL, we have

$$
\begin{array}{r}
\vec{z}, b_{0}^{0}\left[v_{0}\right], b_{1}^{0}\left[v_{1}\right], \text { if } \operatorname{acc}_{0}^{0} \& \operatorname{acc}_{1}^{0} \text { then }\left\langle\mathrm{ub}_{0}^{0}\left[v_{0}\right], \mathrm{ub}_{1}^{0}\left[v_{1}\right]\right\rangle \text { else }\langle\perp, \perp\rangle, \operatorname{not}\left(\operatorname{acc}_{0}^{0} \& \operatorname{acc}_{1}^{0}\right) \\
\text { if } \operatorname{acc}\left(c_{1}^{0}\left[v_{1}\right], t, \mathrm{r}_{\mathrm{b}}^{0}, t_{0}^{1}\right) \& \operatorname{acc}\left(c_{0}^{0}\left[v_{0}\right], t, \mathrm{r}_{\mathrm{b}}^{1}, t_{1}^{1}\right) \\
\sim \vec{z}, \mathrm{~b}\left(c_{1}^{0}\left[v_{1}\right], t, \mathrm{r}_{\mathrm{b}}^{0}\right), \mathrm{b}\left(c_{0}^{0}\left[v_{0}\right], t, \mathrm{r}_{\mathrm{b}}^{1}\right), \\
\text { then }\left\langle\mathrm{ub}\left(c_{0}^{0}\left[v_{0}\right], t, \mathrm{r}_{\mathrm{b}}^{1}, t_{1}^{1}\right), \mathrm{ub}\left(c_{1}^{0}\left[v_{1}\right], t, \mathrm{r}_{\mathrm{b}}^{0}, t_{0}^{1}\right)\right\rangle, \\
\text { else }\langle\perp, \perp\rangle \\
\operatorname{not}\left(\operatorname{acc}\left(c_{1}^{0}\left[v_{1}\right], t, \mathrm{r}_{\mathrm{b}}^{0}, t_{0}^{1}\right) \& \operatorname{acc}\left(c_{0}^{0}\left[v_{0}\right], t, \mathrm{r}_{\mathrm{b}}^{1}, t_{1}^{1}\right)\right)
\end{array}
$$

Using FuncApp, Restr, Proposition 4.3, we get

$$
\begin{aligned}
& \vec{z}, b_{0}^{0}\left[v_{0}\right], b_{1}^{0}\left[v_{1}\right], \operatorname{acc}_{0}^{0} \& \operatorname{acc}_{1}^{0}, \text { if } \operatorname{acc}_{0}^{0} \& \operatorname{acc} c_{1}^{0} \text { then }\left\langle u_{0}^{0}\left[v_{0}\right], u_{1}^{0}\left[v_{1}\right]\right\rangle \text { else }\langle\perp, \perp\rangle \\
& \sim \vec{z}, \mathrm{~b}\left(c_{1}^{0}\left[v_{1}\right], t, \mathrm{r}_{\mathrm{b}}^{0}\right), \mathrm{b}\left(c_{0}^{0}\left[v_{0}\right], t, \mathrm{r}_{\mathrm{b}}^{1}\right), \operatorname{acc}\left(c_{1}^{0}\left[v_{1}\right], t, \mathrm{r}_{\mathrm{b}}^{0}, t_{0}^{1}\right) \& \operatorname{acc}\left(c_{0}^{0}\left[v_{0}\right], t, \mathrm{r}_{\mathrm{b}}^{1}, t_{1}^{1}\right), \\
& \quad \text { if } \operatorname{acc}\left(c_{1}^{0}\left[v_{1}\right], t, \mathrm{r}_{\mathrm{b}}^{0}, t_{0}^{1}\right) \& \operatorname{acc}\left(c_{0}^{0}\left[v_{0}\right], t, \mathrm{r}_{\mathrm{b}}^{1}, t_{1}^{1}\right) \\
& \quad \text { then }\left\langle\left\langle\mathrm{ub}\left(c_{0}^{0}\left[v_{0}\right], t, \mathrm{r}_{\mathrm{b}}^{1}, t_{1}^{1}\right), c_{0}^{0}\left[v_{0}\right], \mathrm{k}_{\mathrm{c}}^{0}\right\rangle,\left\langle\mathrm{ub}\left(c_{1}^{0}\left[v_{1}\right], t, \mathrm{r}_{\mathrm{b}}^{0}, t_{0}^{1}\right), c_{1}^{0}\left[v_{1}\right], \mathrm{k}_{\mathrm{c}}^{1}\right\rangle\right\rangle \\
& \quad \text { else }\langle\perp, \perp\rangle
\end{aligned}
$$

Now, we only need to swap the commitment keys $k_{c}^{0}$ and $k_{c}^{1}$ on the right side. Thanks to ExTFRESHIND which we use to swap the commitment keys on the right side.

Now, we show how the combination of CompHid, CommEQL, UBNotUndef Ined and 
Buindness allow us to fix the inadequacy of the blindness property. The key idea is that as the length of candidate identities are equal, the commitments hide the underlying vote. Thus, the probability that the attacker can cause the blind signature for candidate $v_{b}$ is accepted but rejected for $v_{1-b}$ is negligibly small. This is formalized in the Proposition below.

Proposition $4.8^{1}$ IfEval, IfSame, FuncApp, Trans, IfTrue, IfFalse, Bl indness, UBNotUndef Ined, CompHid, CommEQL, IfMorph, Lemma $4.2 \vdash$

$$
\vec{z}, b_{0}^{0}\left[v_{0}\right], b_{1}^{0}\left[v_{1}\right], t^{0} \sim \vec{z}, b_{0}^{1}\left[v_{1}\right], b_{1}^{1}\left[v_{0}\right], t^{1}
$$

Proof Sketch: The proof idea is the following. Let us further introduce the notation $w_{i}^{j}\left[v_{j \oplus i}\right] \equiv\left\langle\mathrm{ub}_{i}^{j}\left[v_{j \oplus i}\right], c_{i}^{j}\left[v_{j \oplus i}\right]\right\rangle$ where $\oplus$ is XOR. Firstly, in order to match the agent moves from the left to the right, we swap the two inner branches of $t^{1}$ using Lemma 2. Hence,

$$
\begin{array}{r}
t^{1} \equiv \text { if } \operatorname{acc}_{0}^{1} \& \operatorname{acc}_{1}^{1} \text { then }\left\langle\left\langle\mathrm{ub}_{1}^{1}\left[v_{0}\right], c_{1}^{1}\left[v_{0}\right], \mathrm{k}_{\mathrm{c}}^{1}\right\rangle,\left\langle\mathrm{ub}_{0}^{1}\left[v_{1}\right], c_{0}^{1}\left[v_{1}\right], \mathrm{k}_{\mathrm{c}}^{0}\right\rangle\right\rangle \\
\text { else if } \operatorname{acc}_{0}^{1} \text { then }\left\langle\left\langle\mathrm{ub}_{0}^{1}\left[v_{1}\right], c_{0}^{1}\left[v_{1}\right]\right\rangle, \perp\right\rangle \\
\text { else if } \operatorname{acc}_{1}^{1} \text { then }\left\langle\perp,\left\langle\mathrm{ub}_{1}^{1}\left[v_{0}\right], c_{1}^{1}\left[v_{0}\right]\right\rangle\right\rangle \\
\text { else }\langle\perp, \perp\rangle
\end{array}
$$

Now, replace the terms on the then branches as follows:

$$
\begin{aligned}
\left.\left\langle\mathrm{ub}_{0}^{0}\left[v_{0}\right], c_{0}^{0}\left[v_{0}\right], \mathbf{k}_{\mathrm{c}}^{0}\right\rangle,\left\langle\mathrm{ub}_{1}^{0}\left[v_{1}\right], c_{1}^{0}\left[v_{1}\right], \mathbf{k}_{\mathrm{c}}^{1}\right\rangle\right\rangle= & \operatorname{then}\left\langle\left\langle\mathrm{ub}_{0}^{0}\left[v_{0}^{0}\right], c_{0}^{0}\left[v_{0}\right], \mathbf{k}_{\mathrm{c}}^{0}\right\rangle,\left\langle\mathrm{ub}_{1}^{0}\left[v_{1}\right], c_{1}^{0}\left[v_{1}\right], \mathbf{k}_{\mathrm{c}}^{1}\right\rangle\right\rangle \\
& \text { else }\langle\perp, \perp\rangle
\end{aligned}
$$

\footnotetext{
${ }^{1}$ Notice that the proposition in [BCE18] is not sound as it reveals the commitment keys even in the case where only one of the voters obtains the blind signature. Hence, we fixed it here by not revealing the keys in those cases.
} 


$$
\begin{aligned}
\left\langle\left\langle\mathrm{ub}_{1}^{1}\left[v_{0}\right], c_{1}^{1}\left[v_{0}\right], \mathbf{k}_{\mathrm{c}}^{1}\right\rangle,\left\langle\mathrm{ub}_{0}^{1}\left[v_{1}\right], c_{0}^{1}\left[v_{1}\right], \mathbf{k}_{\mathrm{c}}^{0}\right\rangle\right\rangle= & \text { then } \left.\left\langle\left\langle\mathrm{abc}_{1}^{1} \& v_{0}\right], c_{1}^{1}\left[v_{0}\right], \mathbf{k}_{\mathrm{c}}^{1}\right\rangle,\left\langle\mathrm{ub}_{0}^{1}\left[v_{1}\right], c_{0}^{1}\left[v_{1}\right], \mathbf{k}_{\mathrm{c}}^{0}\right\rangle\right\rangle \\
& \text { else }\langle\perp, \perp\rangle
\end{aligned}
$$

Then using the axiom IFBranch, it is sufficient to show the following equivalences:

$$
\begin{gathered}
\vec{z}, b_{0}^{0}\left[v_{0}\right], b_{1}^{0}\left[v_{1}\right], \operatorname{acc}_{0}^{0} \& \operatorname{acc}_{1}^{0}, \text { if } \operatorname{acc}_{0}^{0} \& \operatorname{acc}_{1}^{0} \text { then }\left\langle u_{0}^{0}\left[v_{0}\right], u_{1}^{0}\left[v_{1}\right]\right\rangle \text { else }\langle\perp, \perp\rangle \\
\sim \vec{z}, b_{0}^{1}\left[v_{1}\right], b_{1}^{1}\left[v_{0}\right], \operatorname{acc}_{0}^{1} \& \operatorname{acc}_{1}^{1}, \text { if } \operatorname{acc}_{0}^{1} \& \operatorname{acc}_{1}^{1} \text { then }\left\langle u_{1}^{1}\left[v_{0}\right], u_{0}^{1}\left[v_{1}\right]\right\rangle \text { else }\langle\perp, \perp\rangle \\
\vec{z}, b_{0}^{0}\left[v_{0}\right], b_{1}^{0}\left[v_{1}\right], \operatorname{acc}_{0}^{0} \& \operatorname{acc}_{1}^{0}, \text { if } \operatorname{acc}_{i}^{0} \text { then } w_{i}^{0}\left[v_{i}\right] \text { else } \perp \\
\sim \vec{z}, b_{0}^{1}\left[v_{1}\right], b_{1}^{1}\left[v_{0}\right], \operatorname{acc}_{0}^{1} \& \operatorname{acc}_{1}^{1}, \text { if } \operatorname{acc}_{i}^{1} \text { then } w_{i}^{1}\left[v_{1-i}\right] \text { else } \perp
\end{gathered}
$$

Notice that the equivalence 4.4 has already been proved in Proposition 4.2. The other equivalences 4.5 follow from ExтСомpHid which is defined in Proposition 4.6.

We even further extend the formula 4.3 as shown in the following Proposition:

Proposition 4.9 IfEval, IfSame, FuncApp, Trans, IfTrue, IfFalse, Blindness, UBNotUndef ined, CompHid, CommEQL, IfMorph, Proposition $4.2 \vdash$

$$
\vec{z}, b_{0}^{0}\left[v_{0}\right], b_{1}^{0}\left[v_{1}\right], \operatorname{acc}_{0}^{0}, \operatorname{acc}_{1}^{0}, t^{0} \sim \vec{z}, b_{0}^{1}\left[v_{1}\right], b_{1}^{1}\left[v_{0}\right], \operatorname{acc}_{0}^{1}, \operatorname{acc}_{1}^{1}, t^{1}
$$

Proof Sketch: Recalling the formula 4.3 and using FunCAPP and RESTR, we have,

$$
\vec{z}, b_{0}^{0}\left[v_{0}\right], b_{1}^{0}\left[v_{1}\right], t^{0}, \mathrm{EQ}\left(\pi_{1}\left(\pi_{1}\left(t^{0}\right)\right), \perp\right) \sim \vec{z}, b_{0}^{1}\left[v_{1}\right], b_{1}^{1}\left[v_{0}\right], t^{1}, \mathrm{EQ}\left(\pi_{1}\left(\pi_{1}\left(t^{1}\right)\right), \perp\right)
$$

Now using IfMorph, we have

$$
\vec{z}, b_{0}^{0}\left[v_{0}\right], b_{1}^{0}\left[v_{1}\right], t^{0}, t_{1}^{0} \sim \vec{z}, b_{0}^{1}\left[v_{1}\right], b_{1}^{1}\left[v_{0}\right], t^{1}, t_{1}^{1}
$$




$$
\begin{aligned}
& \text { where } t_{1}^{0} \equiv \text { if } \operatorname{acc}_{0}^{0} \& \operatorname{acc}_{1}^{0} \text { then } \operatorname{EQ}\left(\mathrm{ub}_{0}^{0}\left[v_{0}\right], \perp\right) \\
& \text { else if } \operatorname{acc}_{0}^{0} \text { then } \mathrm{EQ}\left(\mathrm{ub}_{0}^{0}\left[v_{0}\right], \perp\right) \\
& \text { else if } \operatorname{acc}_{1}^{0} \text { then true } \\
& \text { else true } \\
& t_{1}^{1} \equiv \text { if } \operatorname{acc}_{0}^{1} \& \operatorname{acc}_{1}^{1} \text { then } \mathrm{EQ}\left(\mathrm{ub}_{1}^{1}\left[v_{0}\right], \perp\right) \\
& \text { else if } \operatorname{acc}_{0}^{1} \text { then } \operatorname{EQ}\left(\mathrm{ub}_{0}^{1}\left[v_{1}\right], \perp\right) \\
& \text { else if } \operatorname{acc}_{1}^{1} \text { then true } \\
& \text { else true }
\end{aligned}
$$

Using UBNotUndef ined, IfMorph, IfSAme, IfFalse, and FuncApp, we have, $t_{1}^{0} \equiv \operatorname{acc}_{0}^{0}$ and $t_{1}^{0} \equiv \operatorname{acc}_{0}^{1}$.

Similarly, it is not difficult to construct $\operatorname{acc}_{1}^{0}$ on left and $\operatorname{acc}_{1}^{1}$ on the right side.

We show that it is infeasible for an attacker to forge a commitment key with the knowledge of the commitment that was computed using the key in the following proposition.

Proposition 4.10 (Unforgeability of a commitment key) Let $t$ be a ground term and $t_{1}[x]$ be a term that has a single variable $x$. Let $n$ be a name such that $\operatorname{fresh}\left(n ; t, t_{1}\right)$ holds. Then

$$
\mathrm{EQ}\left(t_{1}\left[\operatorname{com}\left(t, \mathrm{k}_{\mathrm{c}}(n)\right)\right], \mathrm{k}_{\mathrm{c}}(n)\right)=\text { false } .
$$

Proof: Let us introduce the notation $c_{1} \equiv \operatorname{com}\left(t, \mathrm{k}_{\mathrm{c}}(n)\right)$ and $c_{2} \equiv \operatorname{com}\left(\operatorname{compl}(t), \mathrm{k}_{\mathrm{c}}\left(n^{\prime}\right)\right)$. Assume the CompHid as the following:

$$
t, \operatorname{compl}(t), c_{1}, c_{2} \sim t, \operatorname{compl}(t), \operatorname{com}\left(\operatorname{compl}(t), \mathrm{k}_{\mathrm{c}}(n)\right), \operatorname{com}\left(t, \mathrm{k}_{\mathrm{c}}\left(n^{\prime}\right)\right)
$$

Then applying RESTR and FunCAPP, we get

$$
t, \operatorname{compl}(t), c_{1} \sim t, \operatorname{compl}(t), \operatorname{com}\left(\operatorname{compl}(t), \mathrm{k}_{\mathrm{c}}(n)\right)
$$


Then using ExtFreshInd and Trans, we have

$$
t, \operatorname{compl}(t), c_{1}, t_{1}\left[c_{1}\right] \sim t, \operatorname{compl}(t), c_{2}, t_{1}\left[c_{2}\right]
$$

Again using FunCAPP and RESTR:

$$
\left.\operatorname{EQ}\left(c_{1}, \operatorname{com}\left(t, t_{1}\left[c_{1}\right]\right)\right) \sim \operatorname{EQ}\left(c_{2}, \operatorname{com}\left(t, t_{1}\left[c_{2}\right]\right)\right)\right)
$$

However, using CompBind, we know that $\left.\mathrm{EQ}\left(c_{2}, \operatorname{com}\left(t, t_{1}\left[c_{2}\right]\right)\right)\right)=$ false.

Hence, the formula 4.7 becomes $\mathrm{EQ}\left(c_{1}, \operatorname{com}\left(t, t_{1}\left[c_{1}\right]\right)\right) \sim$ false. Using the Lemma 5 , we have

$$
\begin{aligned}
& \mathrm{EQ}\left(c_{1}, \operatorname{com}\left(t, t_{1}\left[c_{1}\right]\right)\right)=\text { false } . \\
& \mathrm{EQ}\left(t_{1}\left[c_{1}\right], \mathrm{k}_{\mathrm{c}}(n)\right) \stackrel{\mathrm{IFTF}}{=} \text { if } \mathrm{EQ}\left(t_{1}\left[c_{1}\right], \mathrm{k}_{\mathrm{c}}(n)\right) \text { then true else false } \\
& \stackrel{\mathrm{EQREFL}}{=} \text { if } \mathrm{EQ}\left(c_{1}, \mathrm{k}_{\mathrm{c}}(n)\right) \text { then } \mathrm{EQ}\left(c_{1}, \operatorname{com}\left(t, \mathrm{k}_{\mathrm{c}}(n)\right)\right. \text { else false } \\
& \stackrel{\mathrm{EQBRANCH}}{=} \text { if } \mathrm{EQ}\left(c_{1}, \mathrm{k}_{\mathrm{c}}(n)\right) \text { then } \mathrm{EQ}\left(c_{1}, \operatorname{com}\left(t, t_{1}\left[c_{1}\right]\right)\right) \text { else false } \\
& \text { rewrite } 4.8 \text { if } \mathrm{EQ}\left(c_{1}, \mathrm{k}_{\mathrm{c}}(n)\right) \text { then false else false } \\
& \text { IFSAME false. }
\end{aligned}
$$

\subsection{Proofs of Vote Privacy}

In this section, we prove that the FOO voting protocol satisfies the vote privacy using the CCSA framework for indistinguishability properties. The computational definition of vote privacy of the FOO voting protocol is defined in Section 3.4.1 of 
the Chapter 3. According to the definition, vote privacy holds if it is infeasible for an attacker to distinguish the case where the honest voter $\mathrm{A}$ votes for $v_{0}$ and $\mathrm{B}$ votes for $v_{1}$ from the case where the legitimate voters swap their votes. Of course, both the honest voters must complete all three phases of the protocol and the corresponding votes must appear on the BB.

\subsubsection{Authentication Phase}

It is not hard to see the indistinguishability of the frames of the authentication phase using the computational hiding assumption of the commitments.

\section{Proposition 4.11 (Authentication)}

$$
\vec{z}, b_{0}^{0}\left[v_{0}\right], b_{1}^{0}\left[v_{1}\right] \sim \vec{z}, b_{0}^{1}\left[v_{1}\right], b_{1}^{1}\left[v_{0}\right]
$$

Proof Sketch: Notice that the commitment keys are not revealed in the authentication phase. Hence, using CompHid, FuncApp, and Restr, or ExtCompHid we see that the equivalence holds.

\subsubsection{Voting and Opening Phases}

We consider two possible attacker actions in the voting phase: the attacker may replace one of the ballots and send it to the mixer when he receives the encrypted ballots of the honest voters, and the attacker forwards the received ballots to the mixer without modifying. Here, we prove that the attacker can only win the game with negligible probability in those two scenarios.

Let us introduce the notation $\mathrm{pv}_{i}^{j} \equiv\left\langle c_{i}^{j}\left[v_{j \oplus i}\right], \mathrm{ub}_{i}^{j}\left[v_{j \oplus i}\right], n_{i}\right\rangle$ to denote the ballot that consists of the honest voter's commitment, the accepted unblinded message from the corrupted administrator along with a freshly generated nonce name $n_{i}$. 


\section{Lemma 4.12 (Attacker replaces the ballot of the first voter with his term)}

Suppose that the attacker replaces the ballot of the first voter with a term of his computation in the voting phase. Then

$$
\vec{z}, b_{0}^{0}\left[v_{0}\right], b_{1}^{0}\left[v_{1}\right], t^{0}\left[s^{0}\right] \sim \vec{z}, b_{0}^{1}\left[v_{1}\right], b_{1}^{1}\left[v_{0}\right], t^{1}\left[s^{1}\right]
$$

$$
\begin{aligned}
& \text { where } t^{0}\left[s^{0}\right] \equiv \text { if acco } \& \operatorname{acc}_{1}^{0} \text { then }\left\langle\left\langle e_{0}^{0}\left[v_{0}\right], e_{1}^{0}\left[v_{1}\right], d_{v}\left(v_{1}^{0}, v_{2}^{0}, v_{3}^{0}\right)\left[s^{0}\right]\right\rangle,\left\langle l_{0}^{0}\left[v_{0}\right], l_{1}^{0}\left[v_{1}\right], d_{o}\left(o_{1}^{0}, o_{2}^{0}, o_{3}^{0}\right)\right\rangle\right\rangle \\
& \text { else } \perp \\
& t^{1}\left[s^{1}\right] \equiv \text { if } \operatorname{acc}_{0}^{1} \& \operatorname{acc}_{1}^{1} \text { then }\left\langle\left\langle e_{0}^{1}\left[v_{1}\right], e_{1}^{1}\left[v_{0}\right], d_{v}\left(v_{1}^{1}, v_{2}^{1}, v_{3}^{1}\right)\left[s^{1}\right]\right\rangle,\left\langle l_{0}^{1}\left[v_{1}\right], l_{1}^{1}\left[v_{0}\right], d_{o}\left(o_{1}^{1}, o_{2}^{1}, o_{3}^{1}\right)\right\rangle\right\rangle \\
& \text { else } \perp \\
& \text { if } \operatorname{not}\left(\operatorname{isin}\left(e_{0}^{j}\left[v_{j}\right],\left\langle\tau_{1}\left(f_{2}\left(\Phi_{2}^{j}\right)\right), \tau_{2}\left(f_{2}\left(\Phi_{2}^{j}\right)\right), \tau_{3}\left(f_{2}\left(\Phi_{2}^{j}\right)\right)\right\rangle\right)\right) \\
& s^{j} \equiv \quad \text { then } \operatorname{shufl}\left(\pi_{1}\left(v_{1}^{1}\right), \pi_{1}\left(v_{2}^{1}\right), \pi_{1}\left(v_{3}^{1}\right)\right) \\
& \text { else } 0
\end{aligned}
$$

Proof Sketch: Since the attacker replaces the ballot of the first voter in the voting phase, the voter fails to check his ballot on the Bulletin Board (BB). This is because it is infeasible for the attacker to compute the nonce name $n_{0}$ included in the ballot: Using $\operatorname{ENC}_{\mathrm{CCA} 2}$, we replace the nonce name $n_{0}$ with a fresh variable $n$ in the encrypted ballot $e_{0}^{j}\left[v_{j}\right]$ of the honest voter. Then using FRESHNEQ, we see that EQ $\left(n_{0}, f_{3}\left(\Phi_{3}^{j}\right)\right) \sim$ false for $j=0,1$.

Due to the above reason, in the opening phase, the first voter doesn't reveal his commitment key. The second voter follows the protocol and make sure that his ballot listed on BB. However, the mixer fails to check the condition $\left(\left(\sin _{\mathrm{k}_{\mathrm{c}}^{0}} \&\right.\right.$ $\left.\operatorname{isin}_{\mathbf{k}_{\mathrm{c}}^{1}}\right)$ or $\left(\operatorname{not}\left(\operatorname{isin}_{\mathbf{k}_{\mathrm{c}}^{0}}\right.\right.$ or $\left.\left.\left.\operatorname{isin}_{\mathbf{k}_{\mathrm{c}}^{1}}\right)\right)\right)$ as only the second voter sends the encrypted message $\left\{\left\langle\operatorname{label}\left(c_{1}^{j}\left[v_{j \oplus 1}\right], f_{3}\left(\Phi_{3}^{j}\right)\right), \mathrm{k}_{\mathrm{c}}^{1}, \mathrm{ph}_{3}\right\rangle\right\}_{\mathrm{pk}_{\mathrm{e}}\left(n_{M}\right)}^{\mathrm{r}\left(n_{\mathrm{e}}\right)}$ to the mixer while the first voter skips (outputs $\mathbf{0}$ ) without revealing his commitment key. This is because, it is infeasible for the attacker to forge the commitment key (see Lemma 4.10). Hence the mixer aborts the protocol by outputting $\perp$.

Since the mixer does not reveal the commitment keys in this scenario, we show 
the equivalence 4.17 using ExтCомpHid, which has been defined in Lemma 4.6.

We show that the computational indistinguishability holds when the attacker forwards the honest ballots to the mixer without modifying.

Lemma 4.13 (Attacker replaces none of the honest ballots) Suppose that the attacker replaces none of the ballots of the honest voters. Then

$$
\begin{aligned}
& \vec{z}, b_{0}^{0}\left[v_{0}\right], b_{1}^{0}\left[v_{1}\right], t^{0}\left[s^{0}\right] \sim \vec{z}, b_{0}^{1}\left[v_{1}\right], b_{1}^{1}\left[v_{0}\right], t^{1}\left[s^{1}\right] \\
& t^{0}\left[s^{0}\right] \equiv \text { if } \operatorname{acc}_{0}^{0} \& \operatorname{acc}_{1}^{0} \text { then }\left\langle\left\langle e_{0}^{0}\left[v_{0}\right], e_{1}^{0}\left[v_{1}\right], d_{v}\left(v_{1}^{0}, v_{2}^{0}, v_{3}^{0}\right)\left[s^{0}\right]\right\rangle,\left\langle l_{0}^{0}\left[v_{0}\right], l_{1}^{0}\left[v_{1}\right], d_{o}\left(o_{1}^{0}, o_{2}^{0}, o_{3}^{0}\right)\right\rangle\right\rangle \\
& \text { else } \perp \\
& t^{1}\left[s^{1}\right] \equiv \text { if } \operatorname{acc}_{0}^{1} \& \operatorname{acc}_{1}^{1} \text { then }\left\langle\left\langle e_{0}^{1}\left[v_{1}\right], e_{1}^{1}\left[v_{0}\right], d_{v}\left(v_{1}^{1}, v_{2}^{1}, v_{3}^{1}\right)\left[s^{1}\right]\right\rangle,\left\langle l_{0}^{1}\left[v_{1}\right], l_{1}^{1}\left[v_{0}\right], d_{o}\left(o_{1}^{1}, o_{2}^{1}, o_{3}^{1}\right)\right\rangle\right\rangle \\
& \text { else } \perp \\
& s^{j} \equiv \operatorname{if} \mathrm{EQ}\left(e_{0}^{j}\left[v_{j}\right], x_{1}\right) \& \mathrm{EQ}\left(e_{1}^{j}\left[v_{j \oplus 1}\right], x_{2}\right) \text { then } \operatorname{shufl}\left(\mathrm{pv}_{0}^{j}, \operatorname{pv}_{1}^{j}, \pi_{1}\left(v_{3}^{j}\right)\right) \\
& \text { else if } \mathrm{EQ}\left(e_{0}^{j}\left[v_{j}\right], x_{1}\right) \& \mathrm{EQ}\left(e_{1}^{j}\left[v_{j \oplus 1}\right], x_{3}\right) \text { then } \operatorname{shufl}\left(\mathrm{pv}_{0}^{j}, \pi_{1}\left(v_{2}^{j}\right), \mathrm{pv}_{1}^{j}\right) \\
& \text { else if } \mathrm{EQ}\left(e_{0}^{j}\left[v_{j}\right], x_{2}\right) \& \mathrm{EQ}\left(e_{1}^{j}\left[v_{j \oplus 1}\right], x_{1}\right) \text { then shufl }\left(\mathrm{pv}_{1}^{j}, \mathrm{pv}_{0}^{j}, \pi_{1}\left(v_{3}^{j}\right)\right) \\
& \text { else if } \mathrm{EQ}\left(e_{0}^{j}\left[v_{j}\right], x_{3}\right) \& \mathrm{EQ}\left(e_{1}^{j}\left[v_{j \oplus 1}\right], x_{1}\right) \text { then } \operatorname{shufl}\left(\mathrm{pv}_{1}^{j}, \pi_{1}\left(v_{2}^{j}\right), \mathrm{pv}_{0}^{j}\right) \\
& \text { else if } \mathrm{EQ}\left(e_{0}^{j}\left[v_{j}\right], x_{3}\right) \& \mathrm{EQ}\left(e_{1}^{j}\left[v_{j \oplus 1}\right], x_{2}\right) \text { then } \operatorname{shufl}\left(\pi_{1}\left(v_{1}^{j}\right), \mathrm{pv}_{1}^{j}, \mathrm{pv}_{0}^{j}\right) \\
& \text { else if } \mathrm{EQ}\left(e_{0}^{j}\left[v_{j}\right], x_{2}\right) \& \mathrm{EQ}\left(e_{1}^{j}\left[v_{j \oplus 1}\right], x_{3}\right) \text { then } \operatorname{shufl}\left(\pi_{1}\left(v_{1}^{j}\right), \mathrm{pv}_{0}^{j}, \mathrm{pv}_{1}^{j}\right) \\
& \text { else } \mathbf{0} \\
& x_{i} \equiv \tau_{i}\left(f_{2}\left(\Phi_{2}^{j}\right)\right)
\end{aligned}
$$

Proof Sketch: Firstly, rewrite $l_{0}^{j}\left[v_{j}\right]$ as the following:

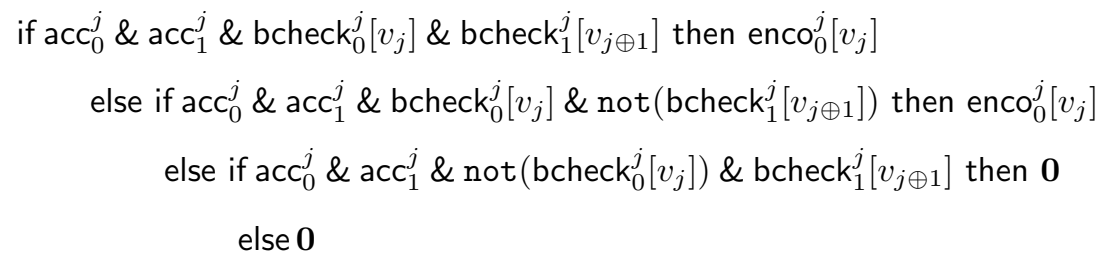


Next, rewrite $l_{1}^{j}\left[v_{j \oplus 1}\right]$ as the following:

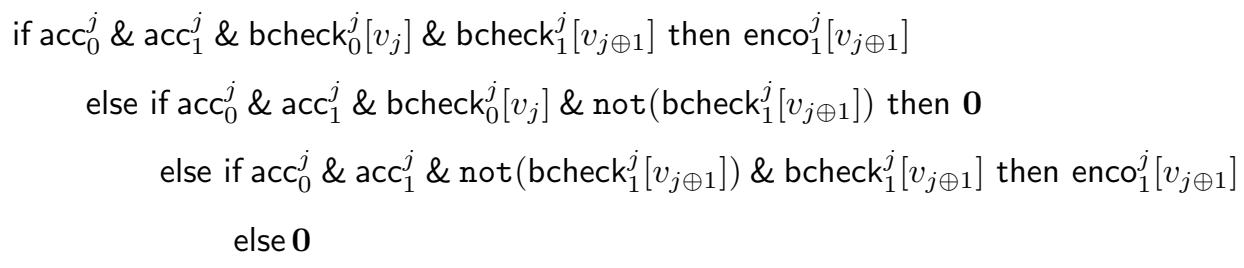

where bcheck ${ }_{i}^{j}\left[v_{j \oplus i}\right] \equiv \operatorname{bnlcheck}\left(c_{i}^{j}\left[v_{j \oplus i}\right], n_{i}, f_{3}\left(\Phi_{3}^{j}\right)\right)$, label ${ }_{i}^{j}\left[v_{j \oplus i}\right] \equiv \operatorname{label}\left(c_{i}^{j}\left[v_{j \oplus i}\right], f_{3}\left(\Phi_{3}^{j}\right)\right)$, and enco ${ }_{i}^{j}\left[v_{j \oplus i}\right] \equiv\left\{\left\langle\operatorname{label}_{i}^{j}\left[v_{j \oplus i}\right], \mathrm{k}_{\mathrm{c}}^{i}, \mathrm{ph}_{3}\right\rangle\right\}_{\mathrm{pk}_{\mathrm{e}}\left(n_{M}\right)}^{\mathrm{r}\left(n_{\mathrm{e} 3+\mathrm{i}}\right)}$. Then, using IFMorPH, lift the conditions of $l_{0}^{j}\left[v_{j}\right]$ and $l_{1}^{j}\left[v_{j \oplus 1}\right]$, and on applying IFBRANCH repeatedly, we have the following equivalences to prove:

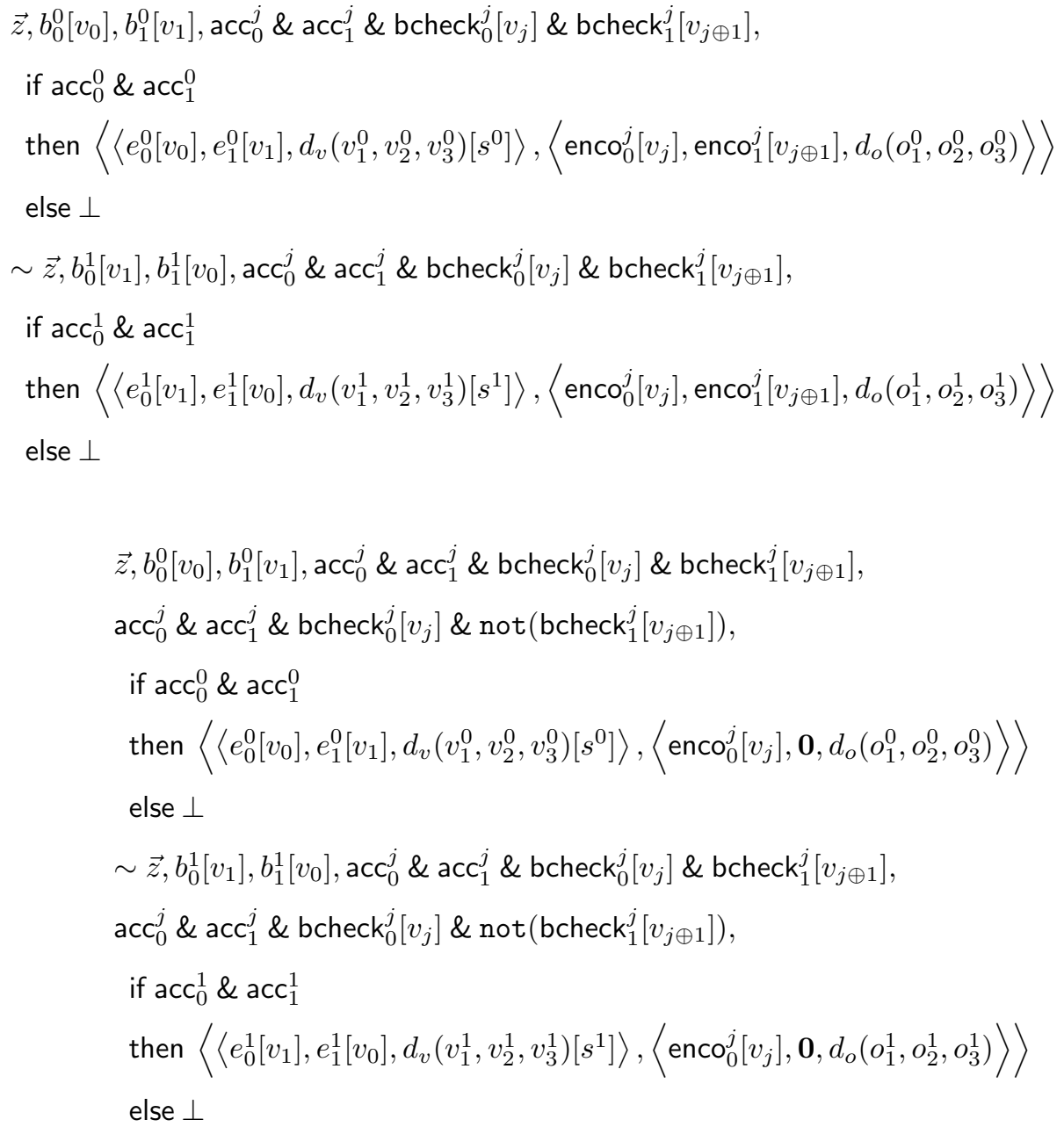




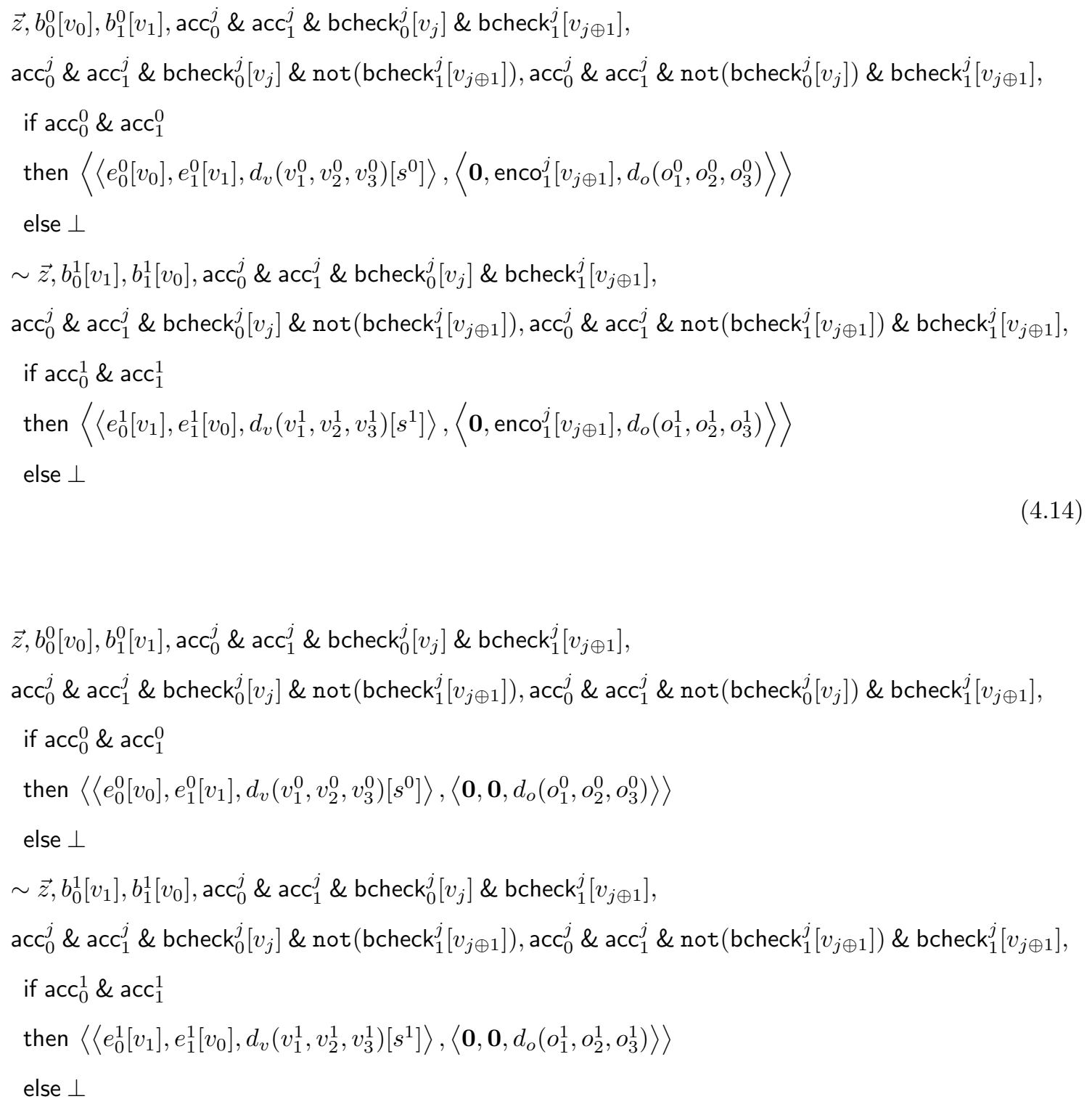

The last three equivalences 4.13,4.14, 4.15 follow from Lemma 4.12 as the mixer term of the opening phase reduced to $\mathbf{0}$.

Now, let us discuss the proof of the equivalence 4.12. Firstly, consider the equiv- 
alence of the Proposition 4.1:

$$
\begin{aligned}
& \vec{z}, b_{0}^{0}\left[v_{0}\right], b_{1}^{0}\left[v_{1}\right], \operatorname{acc}_{0}^{0} \& \operatorname{acc}_{1}^{0}, \text { if } \operatorname{acc}_{0}^{0} \& \operatorname{acc}_{1}^{0} \text { then }\left\langle u_{0}^{0}\left[v_{0}\right], u_{1}^{0}\left[v_{1}\right]\right\rangle \text { else }\langle\perp, \perp\rangle \\
& \sim \vec{z}, b_{0}^{1}\left[v_{1}\right], b_{1}^{1}\left[v_{0}\right], \operatorname{acc}_{0}^{1} \& \operatorname{acc}_{1}^{1}, \text { if } \operatorname{acc}_{0}^{1} \& \operatorname{acc}_{1}^{1} \text { then }\left\langle u_{1}^{1}\left[v_{0}\right], u_{0}^{1}\left[v_{1}\right]\right\rangle \text { else }\langle\perp, \perp\rangle
\end{aligned}
$$

where $u_{i}^{j}\left[v_{j \oplus i}\right] \equiv\left\langle\mathrm{ub}_{i}^{j}\left[v_{j \oplus i}\right], c_{i}^{j}\left[v_{j \oplus i}\right], \mathrm{k}_{\mathrm{c}}^{i}\right\rangle$.

Then on repeated application of an extended version of FuncAPP (see the Proposition 4.3), we see the equivalence which has the commitments in reverse order on the right side. However, we swap the commitments that occur in conditions, shuffling, encryptions using commutativity, SHuFfLE, and ENC $_{\mathrm{CCA} 2}$ axioms respectively on the right side.

Finally, we show that the frames of the opening phase are also indistinguishable to the attacker.

Lemma 4.14 (Opening Phase) Assuming the core axioms, CompHid, CompBind, Blindness, and the additional axioms presented in the Table 3.1, we prove that

$$
\begin{gathered}
\vec{z}, b_{0}^{0}\left[v_{0}\right], b_{1}^{0}\left[v_{1}\right], t^{0}\left[s^{0}\right] \sim \vec{z}, b_{0}^{1}\left[v_{1}\right], b_{1}^{1}\left[v_{0}\right], t^{1}\left[s^{1}\right] \\
t^{0}\left[s^{0}\right] \equiv \quad \text { if acco \& } \operatorname{acc}_{1}^{0} \operatorname{then}\left\langle\left\langle e_{0}^{0}\left[v_{0}\right], e_{1}^{0}\left[v_{1}\right], d_{v}\left(v_{1}^{0}, v_{2}^{0}, v_{3}^{0}\right)\left[s^{0}\right]\right\rangle,\left\langle l_{0}^{0}\left[v_{0}\right], l_{1}^{0}\left[v_{1}\right], d_{o}\left(o_{1}^{0}, o_{2}^{0}, o_{3}^{0}\right)\right\rangle\right\rangle \\
\quad \text { else } \perp \\
t^{1}\left[s^{1}\right] \equiv \text { if acco \& acc } \operatorname{then}\left\langle\left\langle e_{0}^{1}\left[v_{1}\right], e_{1}^{1}\left[v_{0}\right], d_{v}\left(v_{1}^{1}, v_{2}^{1}, v_{3}^{1}\right)\left[s^{1}\right]\right\rangle,\left\langle l_{0}^{1}\left[v_{1}\right], l_{1}^{1}\left[v_{0}\right], d_{o}\left(o_{1}^{1}, o_{2}^{1}, o_{3}^{1}\right)\right\rangle\right\rangle \\
\text { else } \perp
\end{gathered}
$$




$$
\begin{aligned}
& s^{j} \equiv \operatorname{if} \mathrm{EQ}\left(e_{0}^{j}\left[v_{j}\right], x_{1}\right) \& \mathrm{EQ}\left(e_{1}^{j}\left[v_{j \oplus 1}\right], x_{2}\right) \text { then shufl }\left(\mathrm{pv}_{0}^{j}, \mathrm{pv}_{1}^{j}, \pi_{1}\left(v_{3}^{j}\right)\right) \\
& \text { else if } \mathrm{EQ}\left(e_{0}^{j}\left[v_{j}\right], x_{1}\right) \& \mathrm{EQ}\left(e_{1}^{j}\left[v_{j \oplus 1}\right], x_{3}\right) \text { then } \operatorname{shufl}\left(\mathrm{pv}_{0}^{j}, \pi_{1}\left(v_{2}^{j}\right), \mathrm{pv}_{1}^{j}\right) \\
& \text { else if } \mathrm{EQ}\left(e_{0}^{j}\left[v_{j}\right], x_{2}\right) \& \mathrm{EQ}\left(e_{1}^{j}\left[v_{j \oplus 1}\right], x_{1}\right) \text { then } \operatorname{shufl}\left(\mathrm{pv}_{1}^{j}, \mathrm{pv}_{0}^{j}, \pi_{1}\left(v_{3}^{j}\right)\right) \\
& \text { else if } \mathrm{EQ}\left(e_{0}^{j}\left[v_{j}\right], x_{3}\right) \& \mathrm{EQ}\left(e_{1}^{j}\left[v_{j \oplus 1}\right], x_{1}\right) \text { then shufl }\left(\mathrm{pv}_{1}^{j}, \pi_{1}\left(v_{2}^{j}\right), \mathrm{pv}_{0}^{j}\right) \\
& \text { else if } \mathrm{EQ}\left(e_{0}^{j}\left[v_{j}\right], x_{2}\right) \& \mathrm{EQ}\left(e_{1}^{j}\left[v_{j \oplus 1}\right], x_{3}\right) \text { then } \operatorname{shufl}\left(\pi_{1}\left(v_{1}^{j}\right), \mathrm{pv}_{1}^{j}, \mathrm{pv}_{0}^{j}\right) \\
& \text { else if } \mathrm{EQ}\left(e_{0}^{j}\left[v_{j}\right], x_{3}\right) \& \mathrm{EQ}\left(e_{1}^{j}\left[v_{j \oplus 1}\right], x_{2}\right) \text { then } \operatorname{shufl}\left(\pi_{1}\left(v_{1}^{j}\right), \mathrm{pv}_{0}^{j}, \mathrm{pv}_{1}^{j}\right) \\
& \text { else if } n \circ t\left(i \sin \left(e_{0}^{j}\left[v_{j}\right],\left\langle x_{1}, x_{2}, x_{3}\right\rangle\right)\right) \text { then } w^{j} \\
& \text { else } \mathbf{0} \\
& w^{j} \equiv \text { if } \mathrm{EQ}\left(e_{1}^{j}\left[v_{j \oplus 1}\right], x_{1}\right) \text { then } \operatorname{shufl}\left(\mathrm{pv}_{1}^{j}, \pi_{1}\left(v_{2}^{j}\right), \pi_{1}\left(v_{3}^{j}\right)\right) \\
& \text { else if } \mathrm{EQ}\left(e_{1}^{j}\left[v_{j \oplus 1}\right], x_{2}\right) \text { then } \operatorname{shufl}\left(\pi_{1}\left(v_{2}^{j}\right), \mathrm{pv}_{1}^{j}, \pi_{1}\left(v_{3}^{j}\right)\right) \\
& \text { else if } \mathrm{EQ}\left(e_{1}^{j}\left[v_{j \oplus 1}\right], x_{3}\right) \text { then } \operatorname{shufl}\left(\pi_{1}\left(v_{2}^{j}\right), \pi_{1}\left(v_{3}^{j}\right), \mathrm{pv}_{1}^{j}\right) \\
& \text { else if } \operatorname{not}\left(\operatorname{isin}\left(e_{1}^{j}\left[v_{j \oplus 1}\right],\left\langle x_{1}, x_{2}, x_{3}\right\rangle\right)\right) \text { then } \\
& \text { if } \mathrm{EQ}\left(e_{0}^{j}\left[v_{j}\right], x_{1}\right) \text { then shufl }\left(\mathrm{pv}_{0}^{j}, \pi_{1}\left(v_{2}^{j}\right), \pi_{1}\left(v_{3}^{j}\right)\right) \\
& \text { else if } \mathrm{EQ}\left(e_{0}^{j}\left[v_{j}\right], x_{2}\right) \text { then } \operatorname{shufl}\left(\pi_{1}\left(v_{2}^{j}\right), \operatorname{pv}_{0}^{j}, \pi_{1}\left(v_{3}^{j}\right)\right) \\
& \text { else if } \mathrm{EQ}\left(e_{0}^{j}\left[v_{j}\right], x_{3}\right) \text { then } \operatorname{shufl}\left(\pi_{1}\left(v_{2}^{j}\right), \pi_{1}\left(v_{3}^{j}\right), \mathrm{pv}_{0}^{j}\right) \\
& \text { else } \mathbf{0} \\
& x_{i} \equiv \tau_{i}\left(f_{2}\left(\Phi_{2}^{j}\right)\right)
\end{aligned}
$$

Proof Sketch: As explained in the previous lemma, rewrite the terms $l_{0}^{j}\left[v_{j}\right]$ and $l_{1}^{j}\left[v_{j \oplus 1}\right]$. Then lift the conditions of these terms using IFMorph and on the application of IFBRANCH, we get an equivalence that simulates the situation where attacker forwards both the ballots to the mixer in the voting phase and the proof follows from Lemma 4.14.

Again applying IFBRANCH on the else branches we get the equivalences that either simulate the situation where the attacker replaces both the ballots or exactly one of the ballots with his term. Furthermore, these equivalences have some extra conditions on either side of the equality. Since these conditions are just attacker's computations, it is not hard to see that the proofs of these equivalences follow from the proof of Lemma 4.12.

As we saw the indistinguishability of the frames of the authentication, the voting, 
and the opening phases, we conclude that the modified FOO protocol satisfies the vote privacy, assuming the axioms specific to the primitives used in the protocol and other additional assumptions presented in Chapter 3. Hence, we have the following:

Theorem 4.15 (Vote Privacy) The modified FOO protocol respects vote privacy for one session.

\subsection{Summary}

We presented some auxiliary proofs, including the extended versions of the axioms such as FrEShIND, FUnCAPP, and PAIREQL which significantly reduce the number of intermediate steps in the proofs of vote privacy of the FOO protocol. We showed how the combination of CompHid, CommEQL, UBNotUndef Ined, and BLindness allow us to fix the inadequacy of the blindness property. We proved the proofs of vote privacy that the indistinguishability of the frames of the authentication, the voting, and the opening phases of the $\mathrm{FOO}$ voting protocol using the $\mathrm{BC}$ technique for equivalence properties. 


\section{Chapter 5}

\section{Mechanization}

In order to achieve higher assurances in the proofs of vote privacy of the FOO voting protocol, we mechanize them in Coq, an interactive theorem-prover developed in $\left[\mathrm{BC} 04, \mathrm{PdAC}^{+} 17\right]$. We formalize the syntax and the axiomatic system in Coq and utilize the axiomatization to obtain machine-checked proofs of various examples of [BCEO19] and the proofs of vote privacy of the FOO voting protocol. We consider only one session each of responder and initiator to keep the formulas small.

While we were able to translate the proofs directly into Coq, we had to deal with two challenges. The first challenge was posed by the equality predicate $=$ (actually an abbreviation constructed from $\sim$ ) defined between terms, which represents not identity but equality that can fail with negligible probability, and which serves as a congruence in the logic. The native equality relation of Coq forces two terms of our syntax to be equal (in the sense of native Coq equality relation) if and only if they are syntactically identical. This assumption would be unsound for our semantics, where syntactic difference does not necessarily entail inequality. Hence, we define our equality relation and use morphisms $\left[\mathrm{BC} 04, \mathrm{PdAC}^{+} 17\right]$ to model the defined relation is a congruence relation. This allowed us to reuse native Coq tactics such as rewrite and replace seamlessly. The second challenge was posed by the mutual inductiveness 
and nested behaviour of the types message and bool. In such a scenario, Coq generates a weak induction which is not strong enough to prove properties about the types. As explained in Section 3.8 of [Chl13], we strengthen the induction manually and use it in place of the automatically generated one.

Finally, to demonstrate the effectiveness of our mechanization techniques, we formalize the proofs of the authentication and the secrecy of the Authenticated DiffieHellman key exchange protocol which initially appear in the publication [BCEO19] and reproduced in Section 5.3 of this chapter. In order to keep the formulas small, we consider only one session each of responder and initiator. All the machine-checked proofs are available at [Eer19].

\subsection{Formalizing CCSA Framework in Coq}

There are three kinds of specifications that Coq supports, abstract types, mathematical collections, and logical propositions. These specifications are represented by sorts, Type, Set, and Prop respectively.

\subsubsection{Types}

As presented in 2.4, the set of sorts $S$ has at least two sorts message and bool. Using the feature of mutually inductive types [Chl13] in Coq, we define message and bool types as follows. 


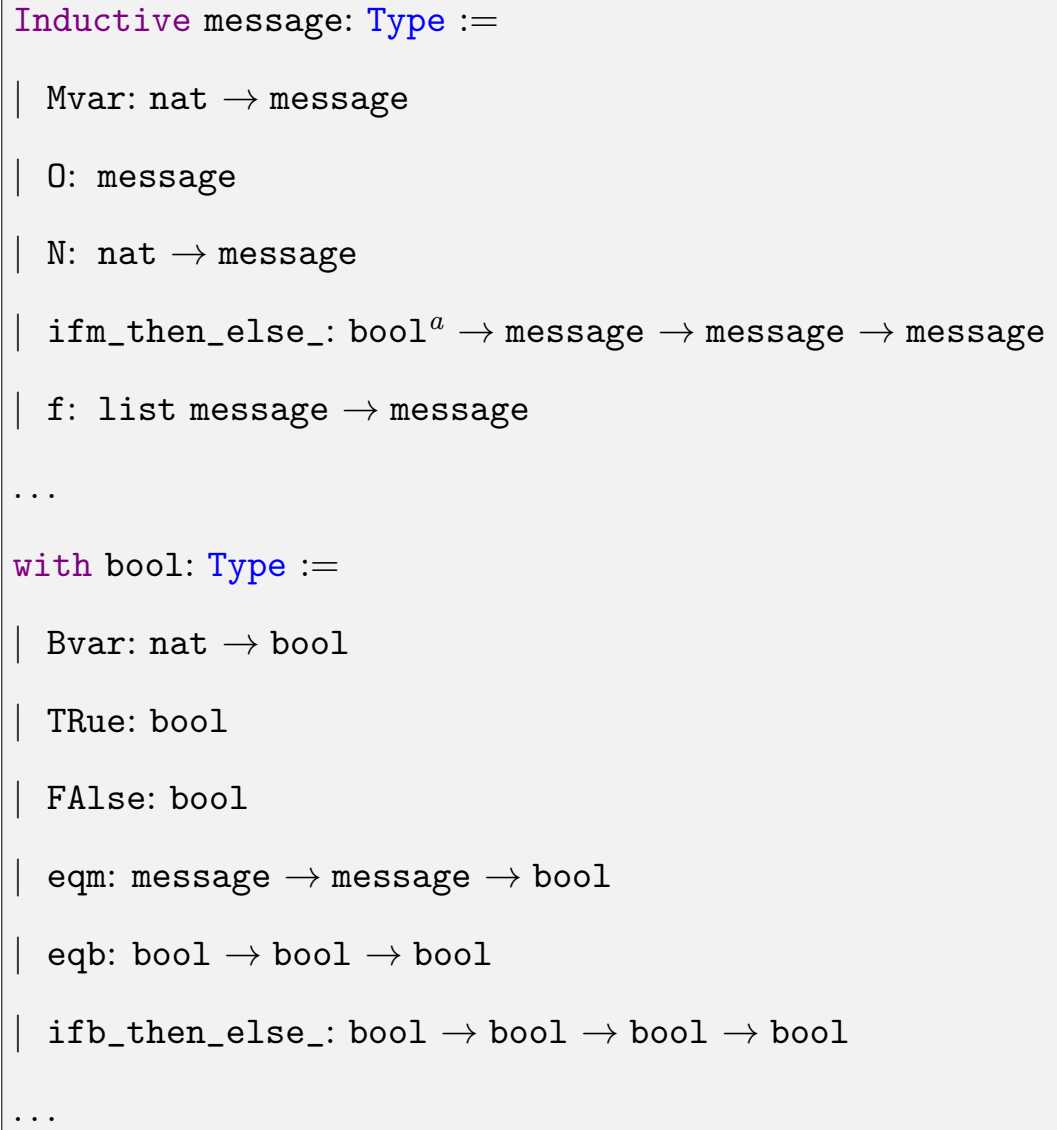

${ }^{a}$ In order to avoid clash with built-in type in Coq, bool is represented as Bool.

As described in [Chl13], ilist is a type that takes length of the list as an argument and produces a length-indexed list. They define polymorphic length-indexed lists. Following [Chl13], we formalize the polymorphic length-indexed list below.

Where the arguments A and nat represent type and length of the ilist respectively. As described in Section 2, list contains message, bool, or both. We needed a type that refers to either of the types. We call it oursum, and is defined inductively on message and bool. 
Inductive oursum: Type :=

msg: message $\rightarrow$ oursum

bol: bool $\rightarrow$ oursum.

A frame is modeled as a length-indexed list of type oursum. This is declared as type mylist $\mathrm{n}$, where $\mathrm{n}$ is length of the list.

Definition mylist: nat $\rightarrow$ Type $:=$ ilist oursum.

\subsubsection{Strengthening Induction}

As message and bool types refer to each other, they are known as mutually inductive types. The induction principles of these types are generated using Scheme command as follows.

Scheme message_Bool_mut := Induction for message Sort Prop

with Bool_message_mut := Induction for Bool Sort Prop.

Invoking Scheme command creates induction principles, message_Bool_mut for the type message and Bool_message_mut for the type bool. Now let us look at the definition of message_Bool_mut using the check command below. 


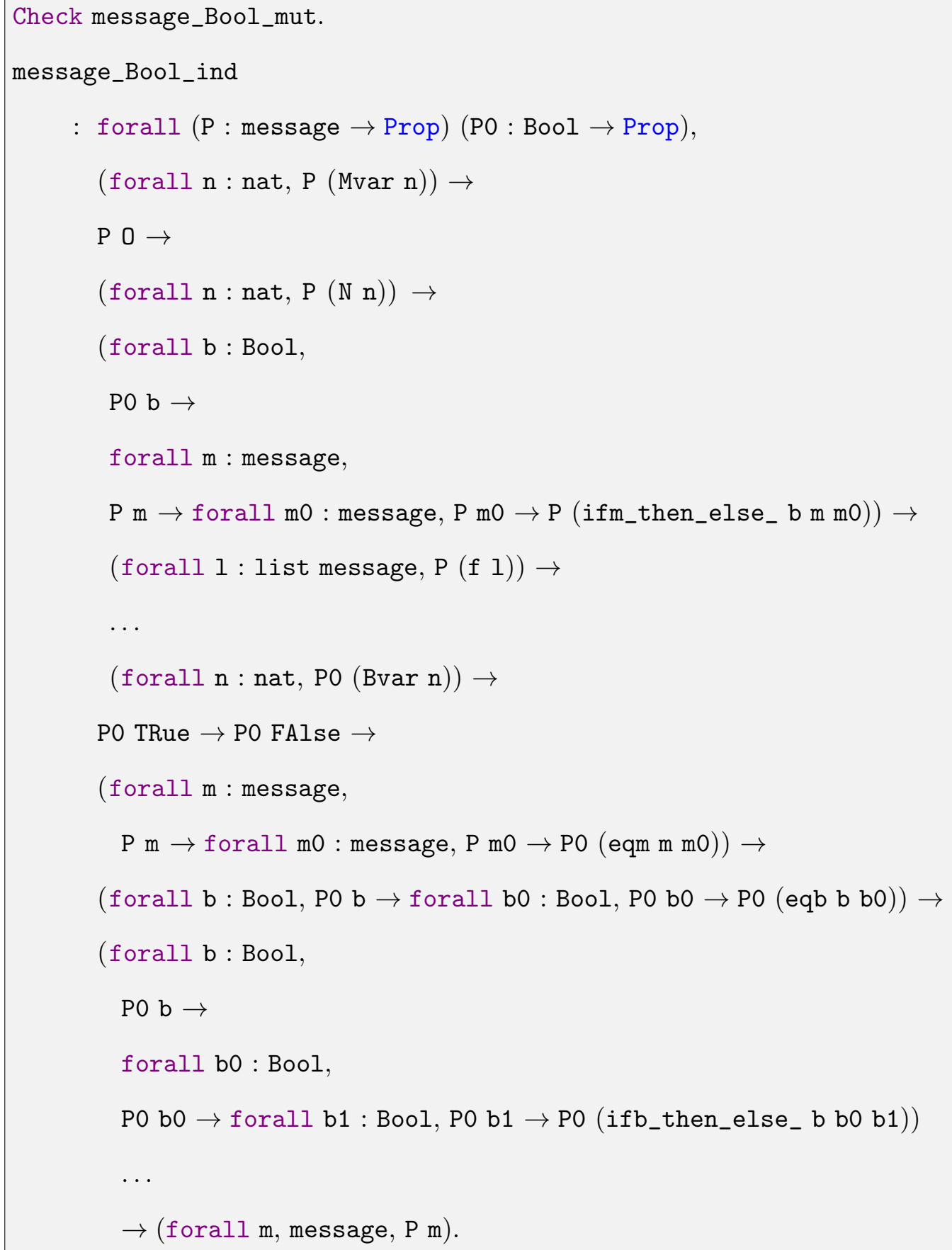

In order to demonstrate the use of these induction principles, we consider proving a simple theorem below. 
Theorem InvarMsg: forall (m:message), (submsg_msg 0 (Mvar 0) m) \#m.

Proof. apply (message_Bool_ind (fun m $\Rightarrow$ (submsg_msg 0 (Mvar 0) m) \#m)

$($ fun $\mathrm{b} \Rightarrow$ (submsg_bol $0($ Mvar 0) b) \#\#b));red_all.

Abort.

Notice that we still have to prove the following goal and the induction principle is not strong enough to reduce the goal:

f (map (submsg_msg n (Mvar n)) l) \#f 1

This is because the types message and bool are mutual and also have nested types as an argument to them. The constructor $f$ of message takes parameterized list type with specified type message as an argument.

As explained in Section 3.8 of [Chl13], we do need to improve the induction principles that are generated using the Scheme command. Towards this, as a first step, we define a unified predicate that represents that a predicate holds for all elements in a list.

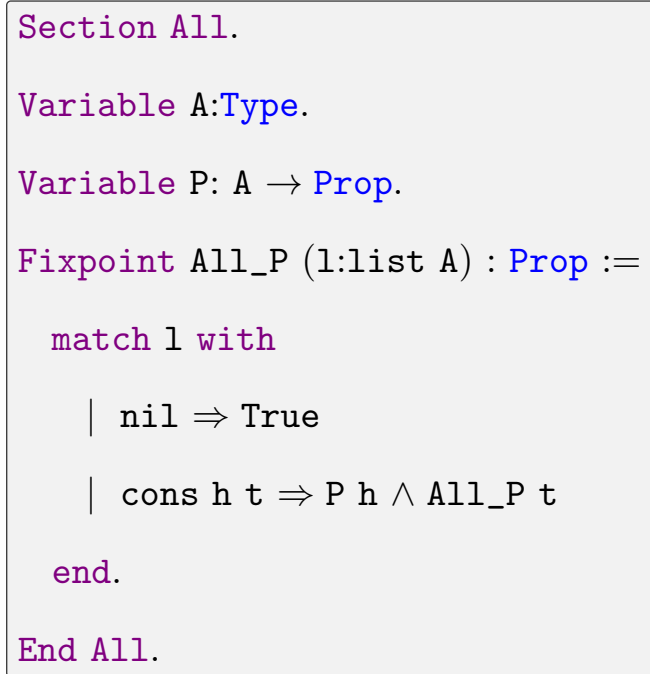

Now we write induction principle that uses nested recursion as follows.

Section ind.

Variable P: message $\rightarrow$ Prop.

Variable PO:Bool $\rightarrow$ Prop. 


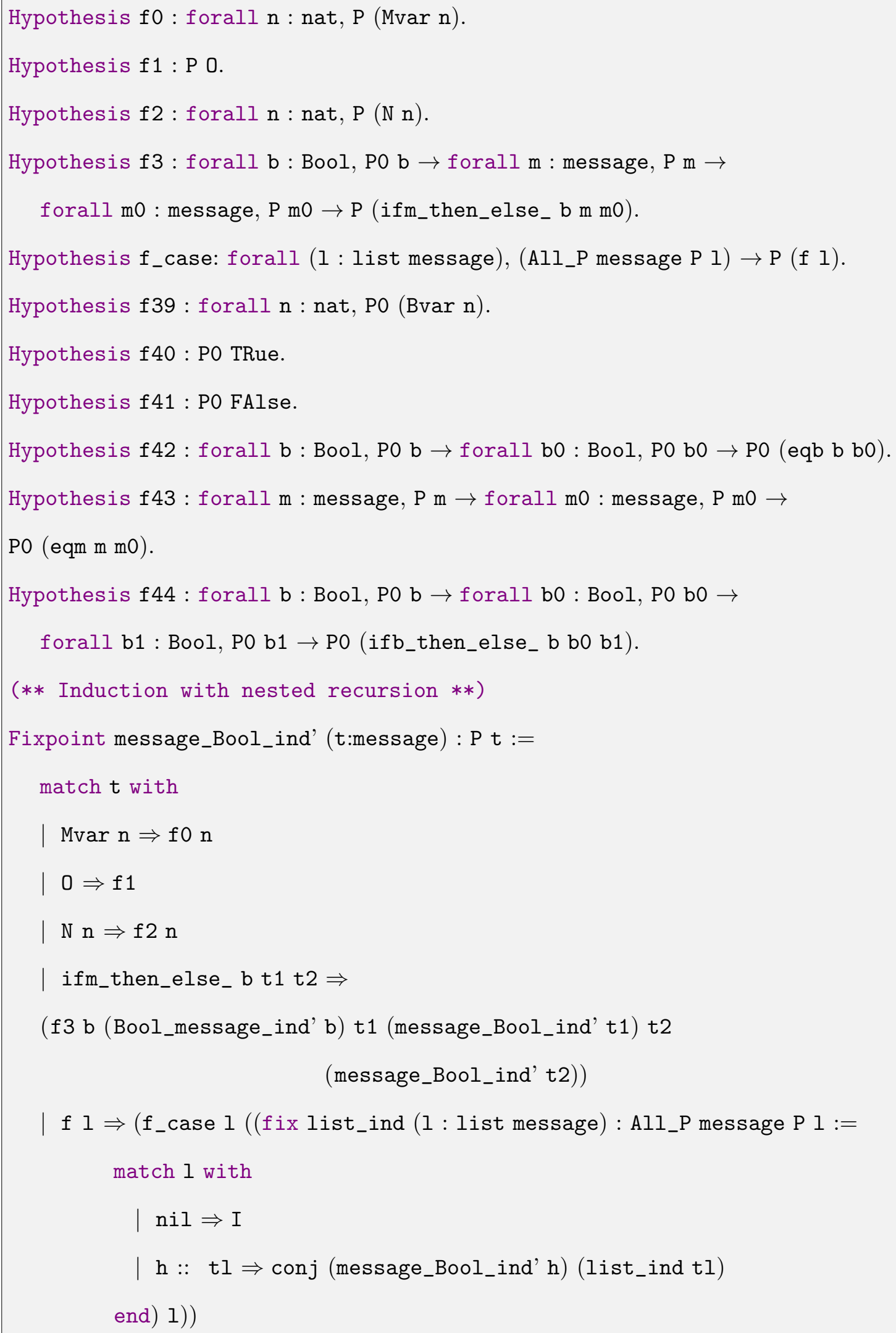




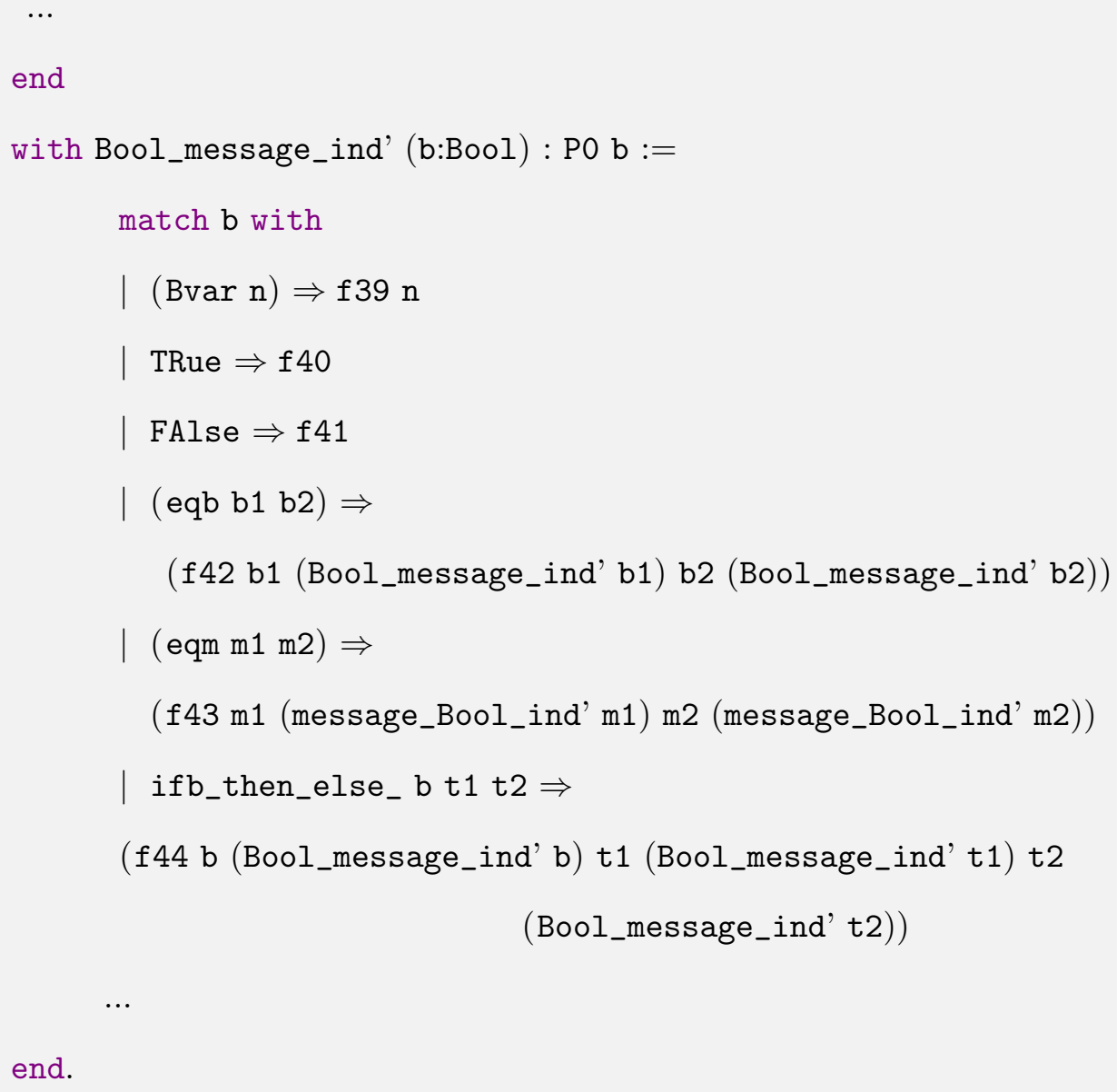

Now we come back to the previous theorem and complete the proof using the improved induction principle.

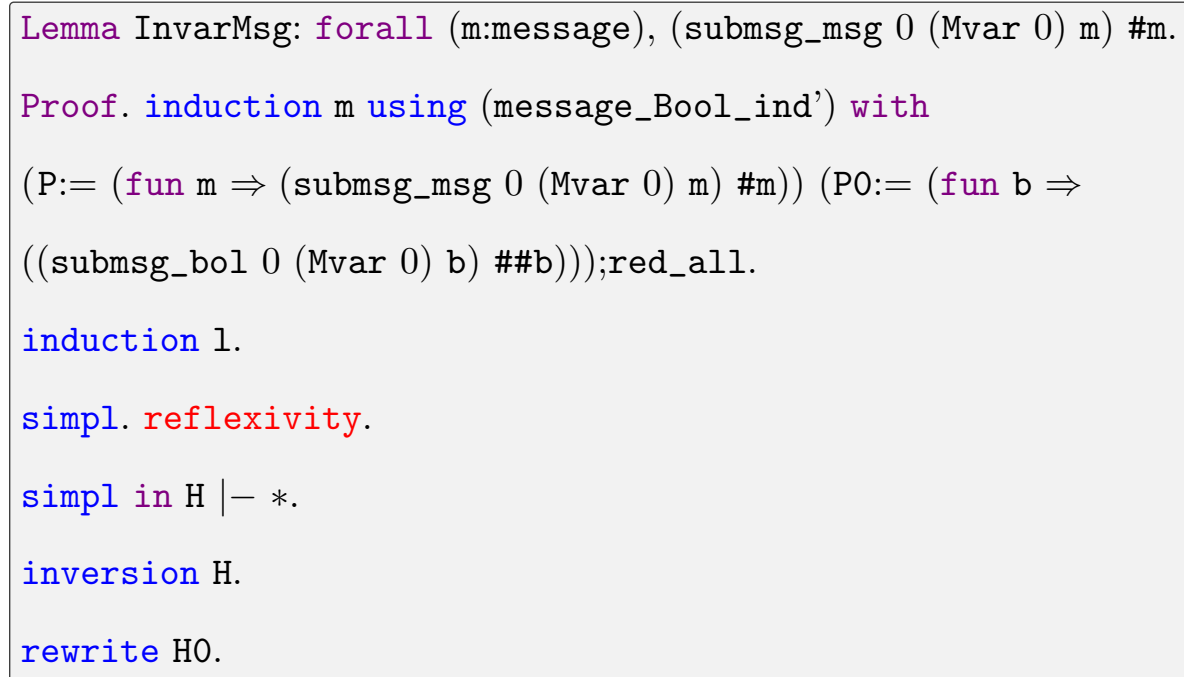


simpl in IHI $\mid-*$.

apply IHI in H1.

simpl.

apply f_cong in H1.

rewrite $\mathrm{H} 1$.

reflexivity. Qed.

\subsubsection{Indistinguishability Relation}

A binary relation on a type $\mathrm{A}$ is formalized in Coq as Definition relation (A:Type) $:=\mathrm{A} \rightarrow \mathrm{A} \rightarrow$ Prop.

We introduce few notations for readability as follows.

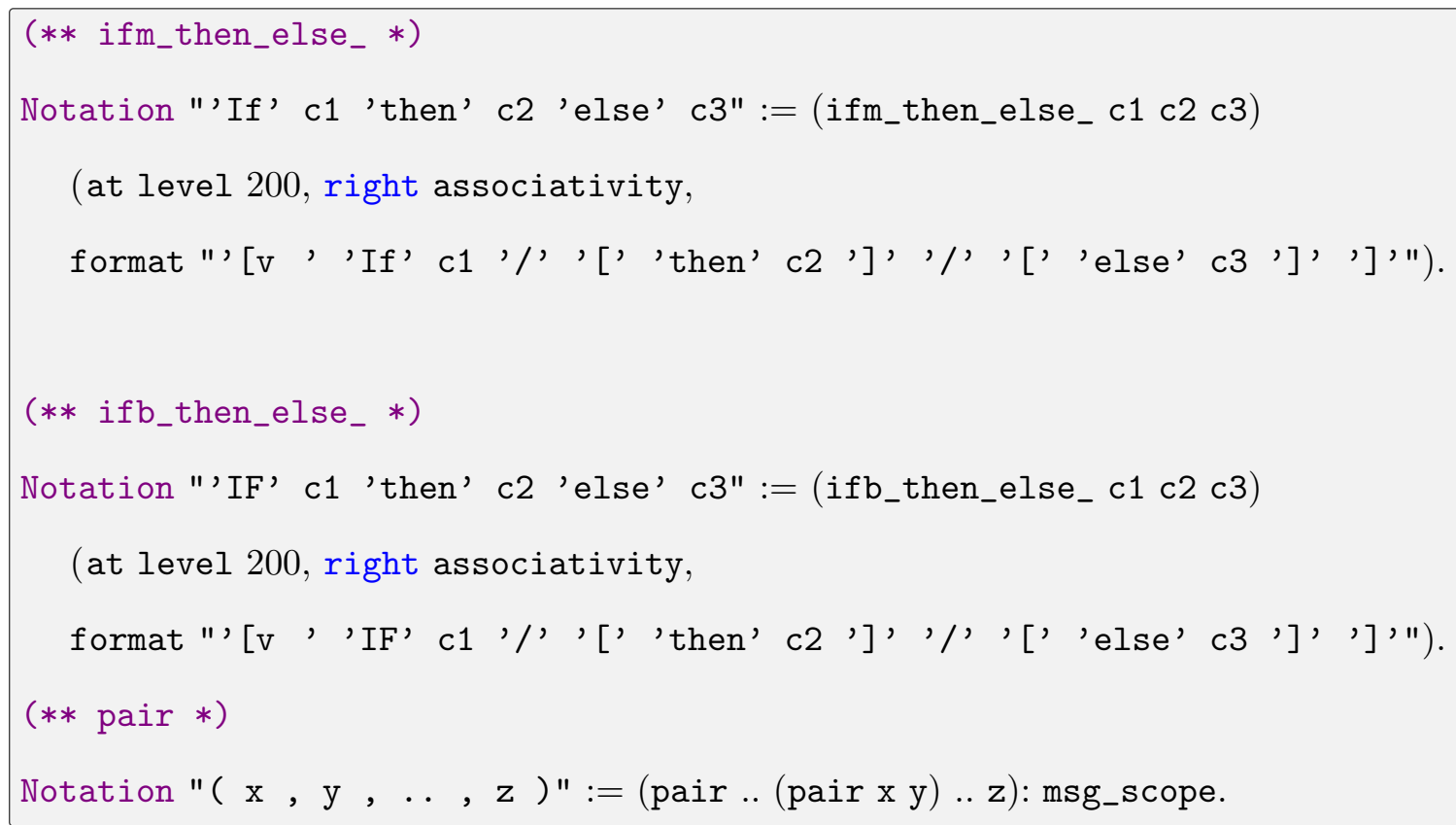

Definition 5.1 (copied verbatim from [BC04, $\left.\mathrm{PdAC}^{+} 17\right]$ ) A parametric relation $R$ is any term of type $\forall\left(x_{1}: T_{1}\right) \ldots\left(x_{n}: T_{n}\right)$, relation $A$. The expression $A$, which depends on $x_{1} \ldots x_{n}$, is called the carrier of the relation and $R$ is said to be a relation over $A$; the list $x_{1}, \ldots, x_{n}$ is the (possibly empty) list of parameters of the 
relation.

We model indistinguishability as a parametric relation on length-indexed lists of type oursum, mylist $\mathrm{n}$, where the length $\mathrm{n}$ is a parameter of the relation. We write the indistinguishability relation as EQI, denoted as $\sim$. The formalization of EQI is achieved using the following command. Parameter EQI: forall n, relation (mylist n).

An instance of a parametric relation EQI with a parameter is a term (EQI $n$ ) where $\mathrm{n}$ is a natural number, and models indistinguishability relation amongst frames of length $\mathrm{n}$. The equivalence property of EQI is formalized as below.

Axiom EQI_equiv: forall n, equiv (mylist n) (@EQI n).

The axiom EQI_equiv states the fact that EQI is an equivalence relation. The properties reflexivity, symmetry, and transitivity of equivalence relation EQI are formalized in the theorems EQI_ref, EQI_sym, and EQI_trans respectively. For example, the theorem EQI_ref is formalized as below.

Theorem EQI_ref: forall \{n:nat\} (ml: mylist n), $\mathrm{ml} \sim \mathrm{ml}$.

Similarly, other theorems are formalized. The parametric equivalence relation EQI can be declared with the following command.

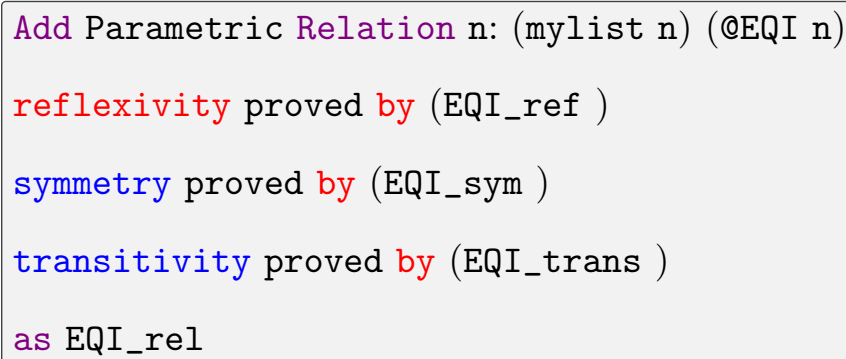

Where the name EQI_rel uniquely identifies the relation and is used to generate fresh names, EQI_rel_Reflexive, EQI_rel_Symmetric, for automatically provided lemmas. 


\subsubsection{Formalization of Equality}

Recall the abbreviation $=$ defined in the syntax Section of the paper [BCEO19], which serves as a congruence. If we use the built-in equality of Coq to model this abbreviation together with its built-in axiomatic system, then we end up with an unsound extension of the Coq logic. This is because the Coq inequality forces two syntactically unequal terms of our logic to be unequal which will contradict our axioms (for example, IFTRUE). Instead, we model $=$ as a pair of equivalence relations: EQm for modeling $=$ amongst terms of type message and EQb for modeling $=$ amongst terms of type bool.

The formalization of EQm and EQb is achieved with the following commands:

Definition EQm: relation message :=

$$
\text { fun }(\mathrm{m} 1 \mathrm{~m} 2: \text { message }) \Rightarrow[\text { bol (eqm m1 m2) }] \sim[\text { bol TRue }] \text {. }
$$

Definition EQb: relation bool :=

fun $(\mathrm{b} 1 \mathrm{~b} 2:$ bool $) \Rightarrow[$ bol (eqb b1 b2) $] \sim[$ bol TRue $]$.

For the rest of the paper, we write EQm as \# and EQb as \#\#.

Even if we do not use the native $=$, we still would like to use native Coq tactics which exploit the fact that Leibniz equality is a congruence. Thus, we declare EQm and EQb as morphisms which allows us to exploit their congruence properties.

The tactic rewrite term $\left[\mathrm{BC} 04, \mathrm{PdAC}^{+} 17\right]$, where type of the term is of the form forall (x1:A1) ...(xn:An) eq term1 term2

where eq is the Leibniz equality or a registered user-defined equality, applies to any goal. It finds instances of term1 by matching it in the goal and replaces them with instances of term2. Of course, some of the variables xi solved by unification and some of the types $\mathrm{A} 1, \ldots$, An become new subgoals. The variants of the tactic are also defined. For more details about the tactic readers are directed to $\left[\mathrm{BC} 04, \mathrm{PdAC}^{+} 17\right]$.

The tactic replace term1 with term2 applies to any goal, and it replaces all 
occurrences of term1 with term2 and generates a subgoal term1 = term2. Then sub goal is solved if it or its symmetric form occurs as an assumption in the context. Of course, variants of it is also defined and readers are directed to $\left[\mathrm{BC} 04, \mathrm{PdAC}^{+} 17\right]$ for more information.

Now, we define morphisms for the functions that respect the relations EQm and EQb respectively.

Example 5.2 We have discussed syntactic equality of lists of type A in the example 11.1. We define a function app_list: forall $\mathrm{A}$, list $\mathrm{A} \rightarrow$ list $\mathrm{A} \rightarrow$ list $\mathrm{A}$, concatenation of two lists of type A, as appending a list of type A to other. We also formalize the fact that appending an empty list to any other list gives the same list. Axiom empty_app: forall (A:Type) (1: list A), list_eq (app_list 1 []) 1.

Notice that the built-in tactic rewrite do not replace terms with related one inside the function app_list over the relation list_eq. For example, consider proving the following goal.

Goal ex forall ( l: list nat), (list_eq (app_list (app_list l []) l) (app_list l l)). Proof. intros. rewrite empty_app. Abort.

The rewrite tactic does not work and we abort the goal for now.

Definition 5.3 (Morphism (copied verbatim from [BC04, $\left.\mathrm{PdAC}^{+} 17\right]$ )) A parametric unary function $f$ of type forall $\left(x_{1}: T_{1}\right) \ldots\left(x_{n}: T_{n}\right), A_{1} \rightarrow A_{2}$ covariantly respects two parametric relation instances $R_{1}$ and $R_{2}$ if, whenever $x, y$ satisfy $R_{1} x y$, their images $(f x)$ and $(f y)$ satisfy $R_{2}(f x)(f y)$. An $f$ that respects its input and output relations is called a unary covariant morphism. The sequence $x_{1}, \ldots, x_{n}$ represents the parameters of the morphism.

Example 5.4 The constructor pair takes two terms of type message and gives a term of type message. We have the following axiom: 
Axiom pair_Cong: forall (m1 m2 m1' m2': message), m1 \#m1' $\rightarrow \mathrm{m} 2$ \# m2' $\rightarrow$

$(\mathrm{m} 1, \mathrm{~m} 2) \#\left(\mathrm{~m} 1^{\prime}, \mathrm{m} 2{ }^{\prime}\right)$.

We add a morphism pair_mor of the function pair that respects the relation EQm as follows. The morphism pair_mor is declared using the following commands.

Add Parametric Morphism: (@ pair) with

signature $\mathrm{EQm} \Rightarrow \mathrm{EQm} \Rightarrow \mathrm{EQm}$ as pair_mor.

Proof. intros. apply pair_Cong; assumption. Qed.

The command declares pair as a morphism of the signature EQm $\Rightarrow$ EQm $\Rightarrow$ EQm. The identifier app_mor gives a unique name to the morphism. The command also requires us to prove that the function pair respects the relations identified by the signature. The proof uses the axiom pair_Cong. Similarly, morphisms for other functions are formalized.

\subsubsection{Formalization of the Core Axioms}

The formalization of the core axioms (see Table 2.1), the axioms for indistinguishability, equality, if _then_else _ symbol, and the names, of the BC framework is presented below.

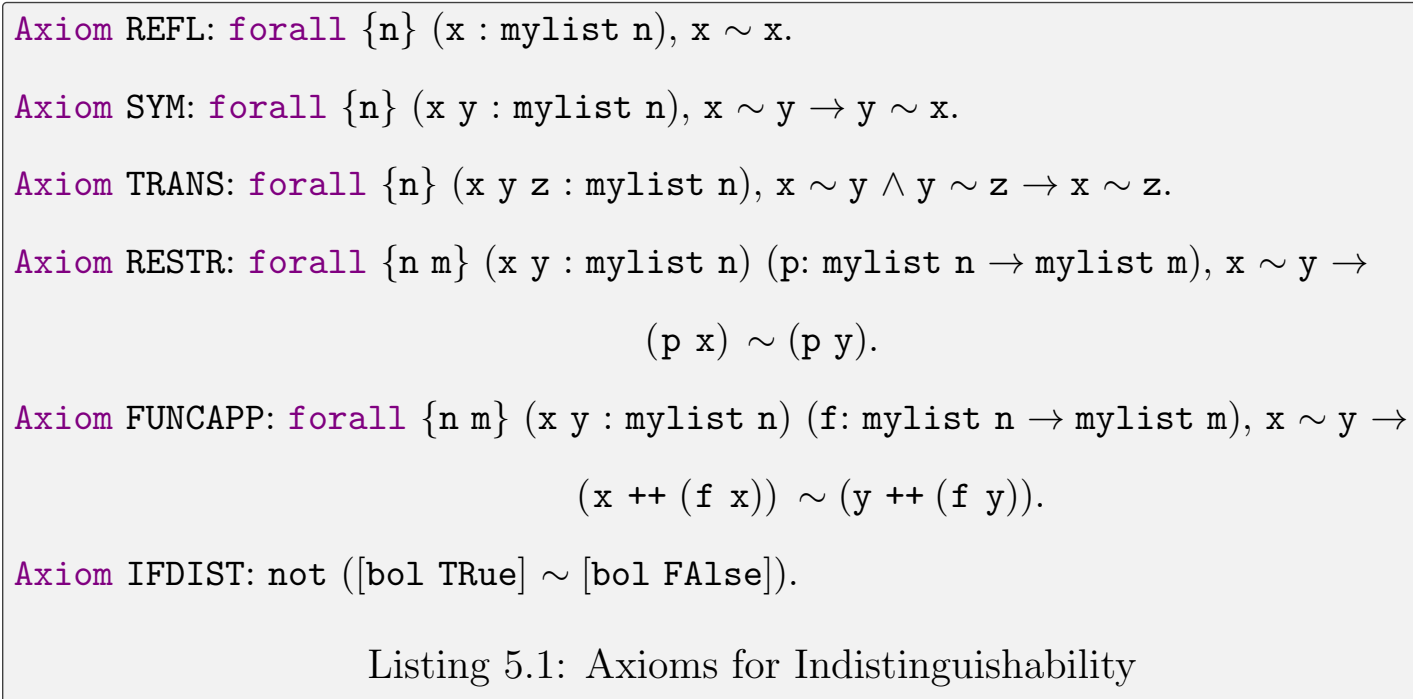

Listing 5.1: Axioms for Indistinguishability

The axioms of equality include the EqCong and EqTheo. We duplicate the axioms 
to distinguish the types message and bool.

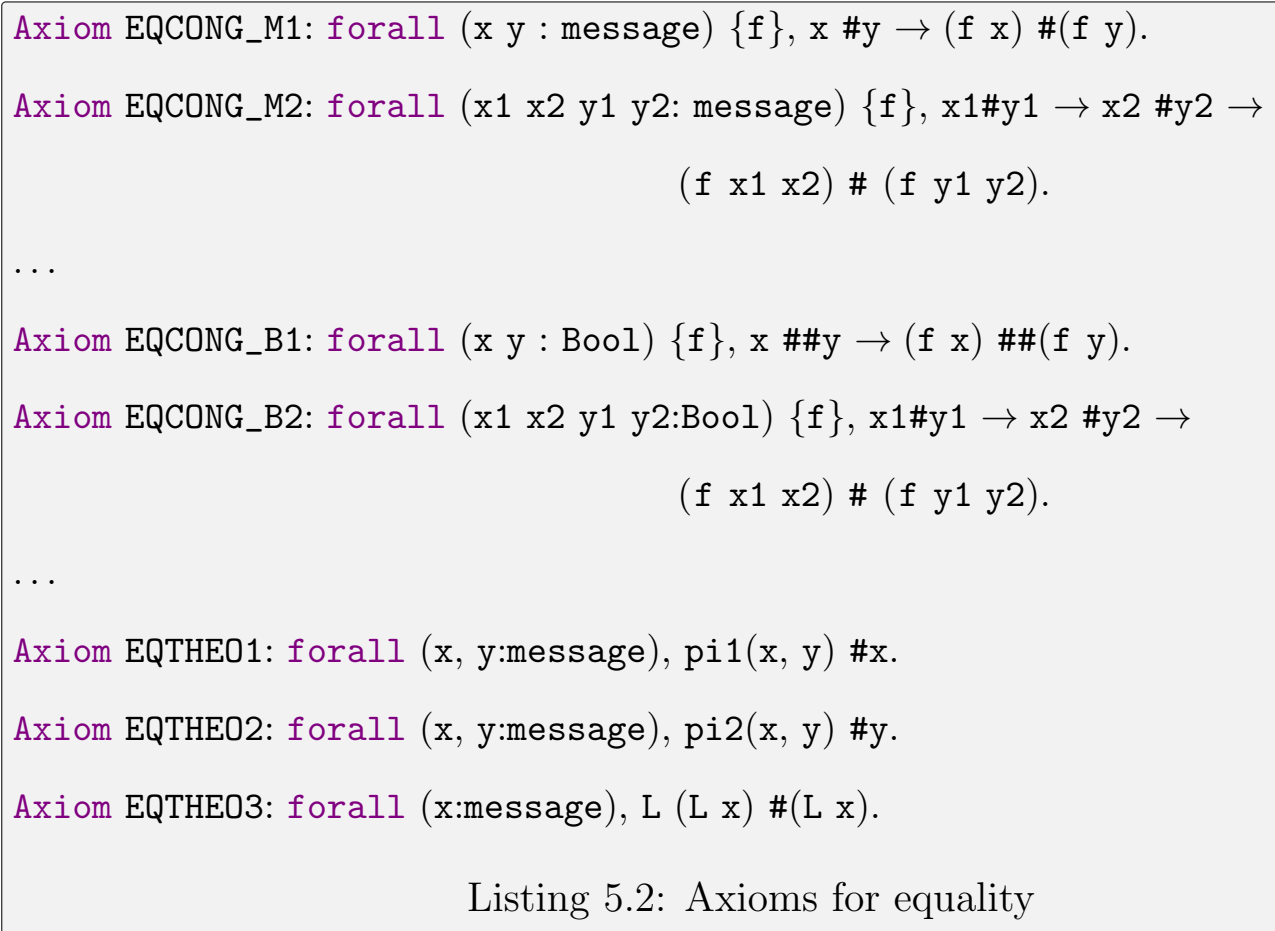

Listing 5.2: Axioms for equality

We present the formalization of the axioms for the if _then _ else _ symbol below.

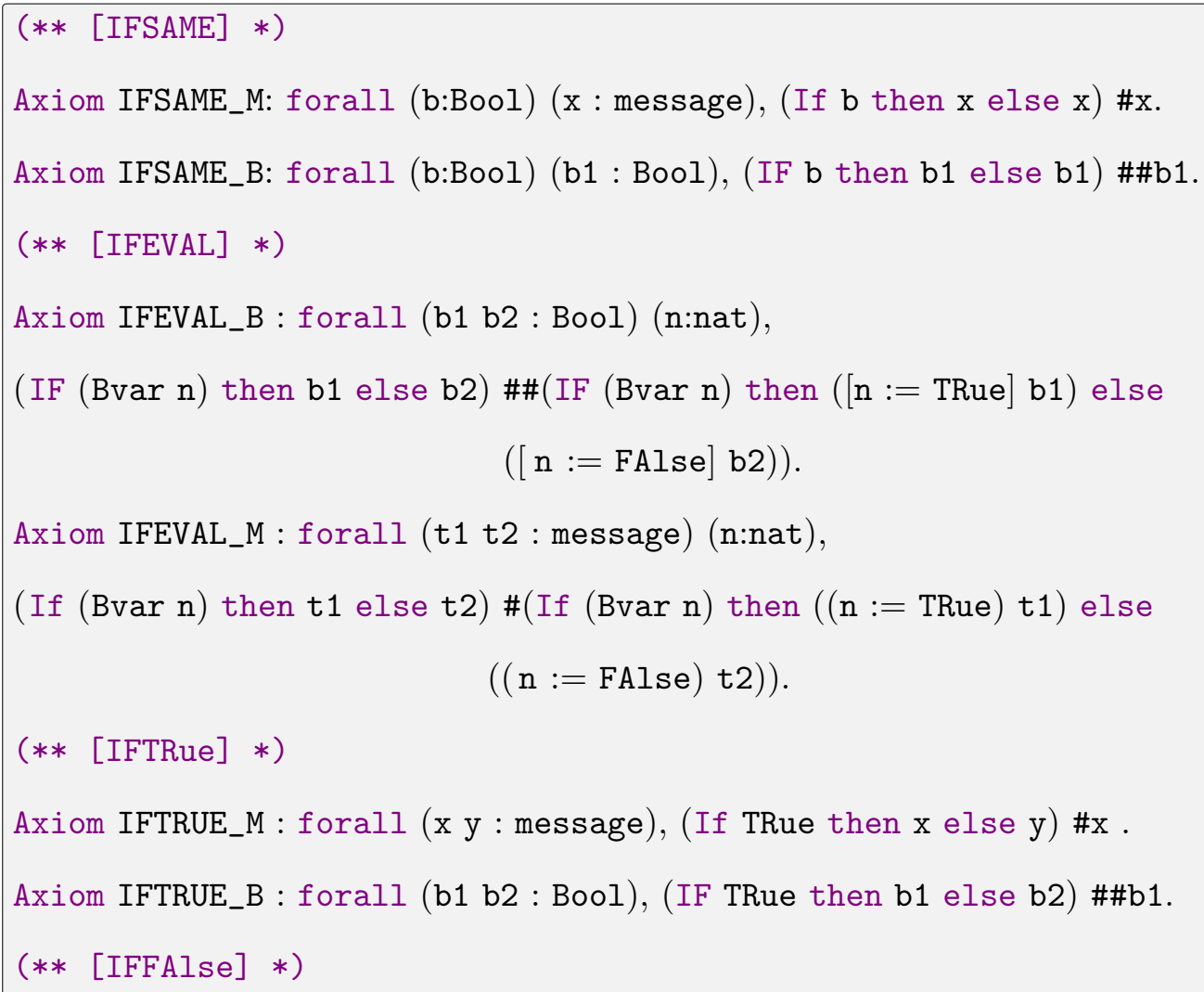


Axiom IFFALSE_M: forall (x y : message), (If FAlse then $\mathrm{x}$ else y) \#y.

Axiom IFFALSE_B: forall (b1 b2 : Bool), (IF FAlse then b1 else b2) \#\#b2.

$(* * \quad$ IFBRANCH $*)$

Axiom IFBRANCH_M: forall (n: nat) (ml1 ml2 : mylist n) (b b' : Bool)

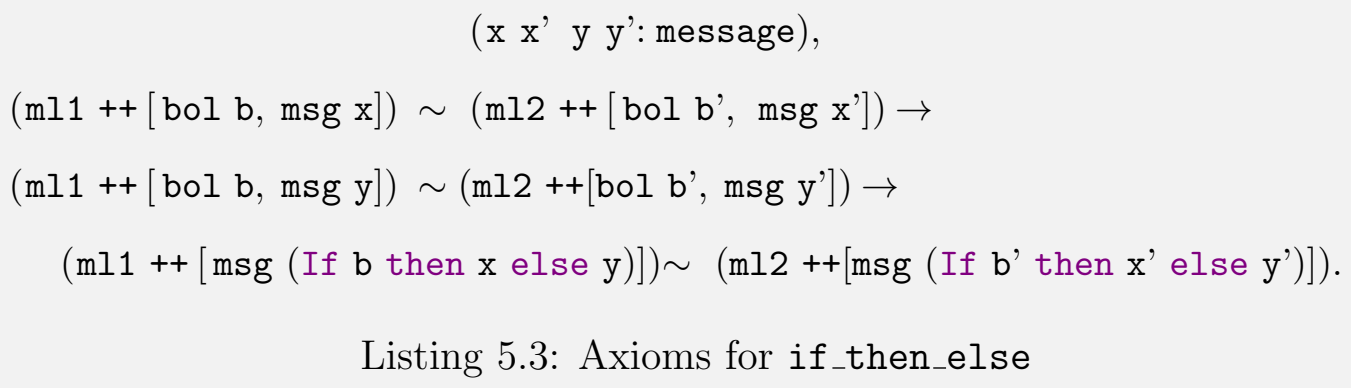

Finally, we present the formalization of the axioms for the names below.

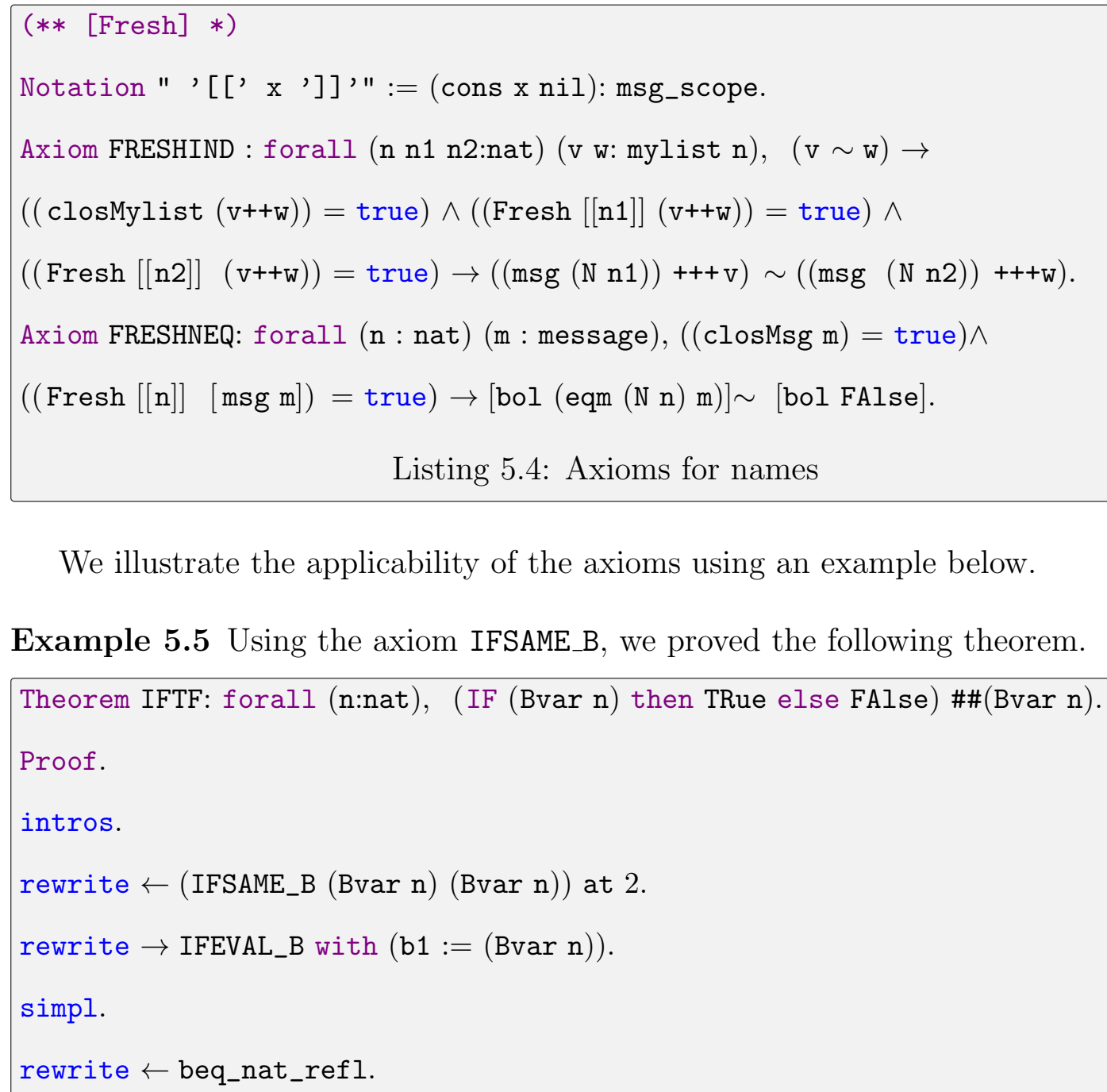

We illustrate the applicability of the axioms using an example below.

Example 5.5 Using the axiom IFSAME_B, we proved the following theorem. 
reflexivity.

Qed.

where beq_nat_refl is a lemma that states, for any natural number $\mathrm{n}$, we have true $=$ (beq_nat $\mathrm{n} n$ ).

\subsection{Formalizing the Proofs of the Vote Privacy}

To achieve higher assurances on our analysis, we formalize the proofs, presented in Chapter 4, of vote privacy of the FOO voting protocol in Coq.

We formalize the Proposition 4.1 which extends the Blindness axiom below.

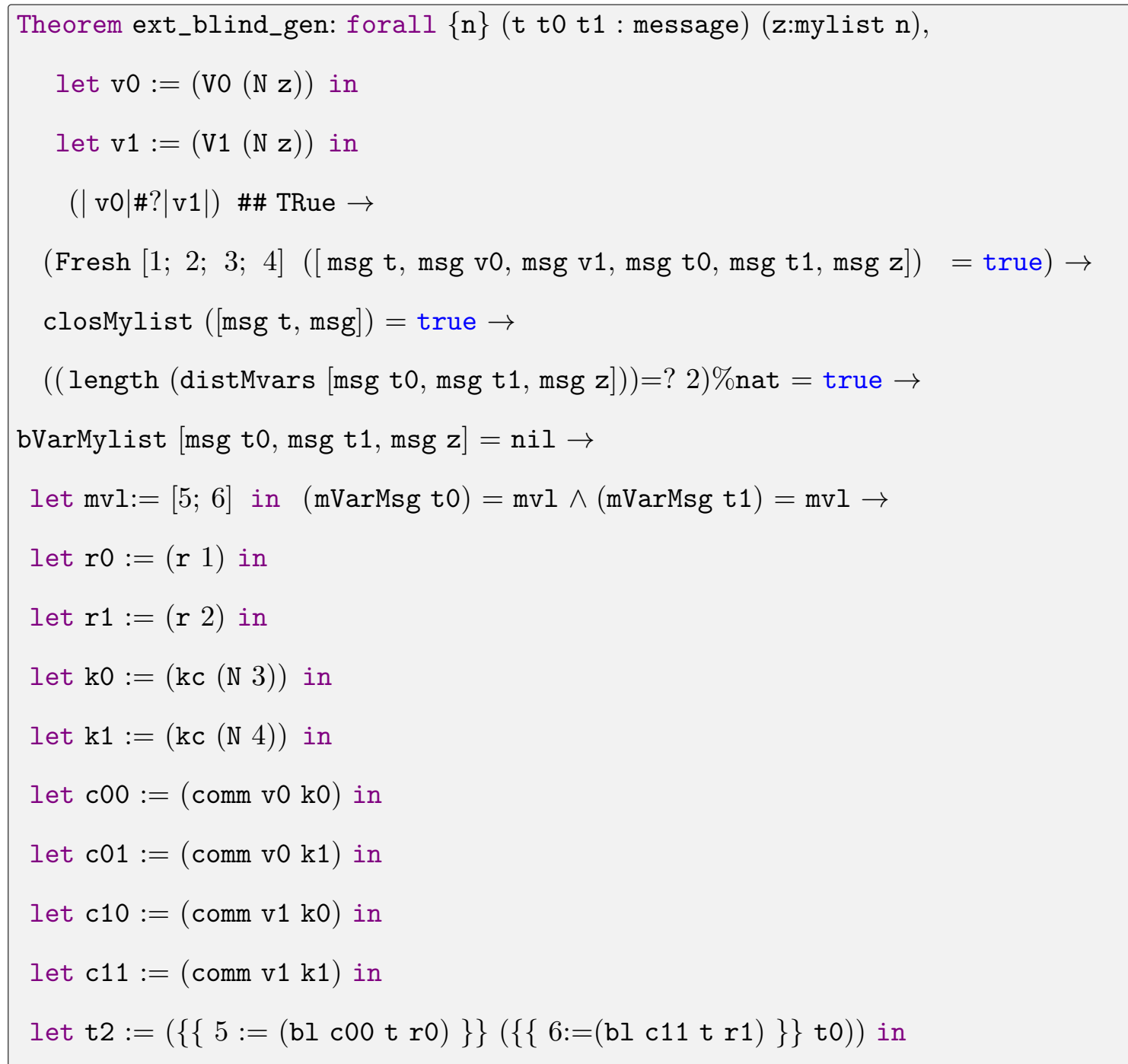




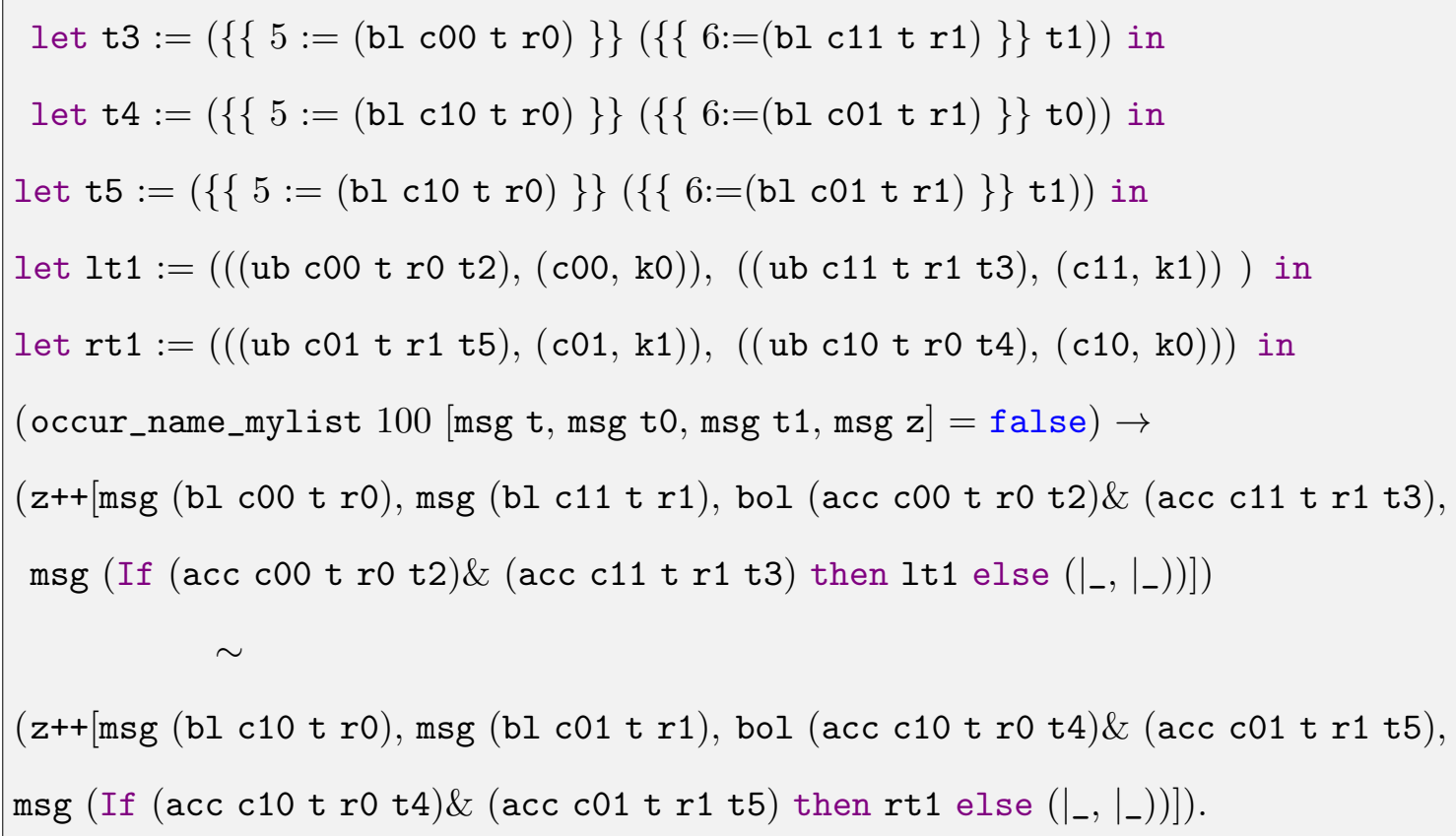

We formalize the Proposition 4.8 in which we show how the combination of CompHid, CommEQL, UBNotUndefined and BLindness allow us to fix the inadequacy of the blindness property below. The key idea is that as the length of candidate identities are equal, the commitments hide the underlying vote. Thus, the probability that the attacker can cause the blind signature for candidate $v_{b}$ is accepted but rejected for $v_{1-b}$ is negligibly small.

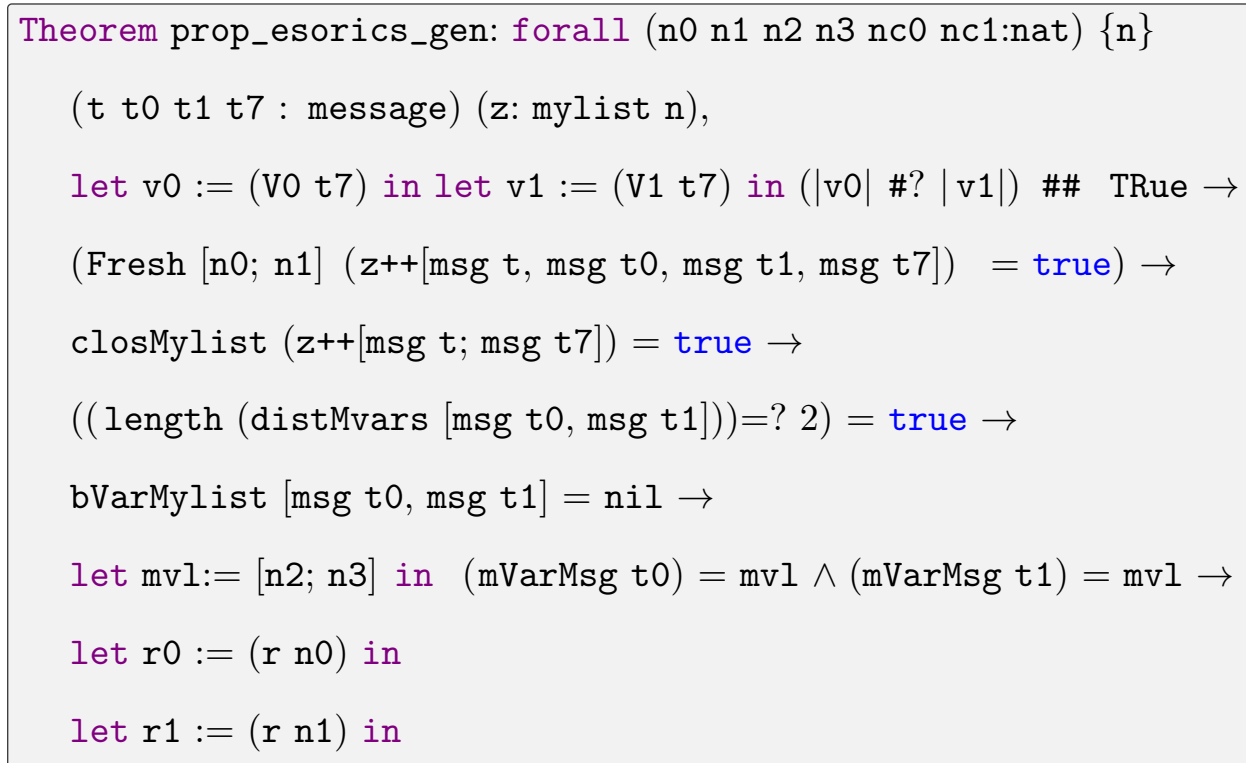


let $\mathrm{k} 0:=(\mathrm{kc}(\mathrm{N} \mathrm{nc} 0))$ in

let $\mathrm{k} 1:=(\mathrm{kc}(\mathrm{N} \mathrm{nc1)})$ in

let $\mathrm{c} 0:=($ comm v0 $\mathrm{k} 0)$ in

let $\mathrm{c0}^{\prime}:=($ comm v0 k1) in

let $\mathrm{c} 1:=($ comm v1 $\mathrm{k} 0)$ in

let $\mathrm{c}^{\prime}:=($ comm $\mathrm{v} 1 \mathrm{k} 1)$ in

let $\mathrm{t} 2:=(\{\{\mathrm{n} 2:=(\mathrm{bl}$ c0 $\mathrm{t} \mathrm{r} 0)\}\}(\{\{\mathrm{n} 3:=(\mathrm{bl} \mathrm{c} 1 \mathrm{t} \mathrm{r} 1)\}\}$ to $))$ in

let $\mathrm{t} 3:=(\{\{\mathrm{n} 2:=(\mathrm{bl}$ co t $\mathrm{r} 0)\}\}(\{\{\mathrm{n} 3:=(\mathrm{bl} \mathrm{c} 1 \mathrm{t} \mathrm{r} 1)\}\}$ t 1$))$ in

let $\mathrm{t} 4:=(\{\{\mathrm{n} 2:=(\mathrm{bl} c 1$ t $\mathrm{r} 0)\}\}(\{\{\mathrm{n} 3:=(\mathrm{bl} \mathrm{cot} r 1)\}\}$ to $))$ in

let $\mathrm{t} 5:=(\{\{\mathrm{n} 2:=(\mathrm{bl} \mathrm{c} 1 \mathrm{t} \mathrm{r} 0)\}\}(\{\{\mathrm{n} 3:=(\mathrm{bl} \mathrm{cot} r 1)\}\} \mathrm{t} 1))$ in

let $l t 1:=\left(((u b c 0\right.$ t r0 t2 $),(c 0, k 0)),\left((u b c 1 ' t r 1\right.$ t2 $\left.\left.),\left(c 1^{\prime}, k 1\right)\right)\right)$ in

let $1 \mathrm{t} 2:=\left((\mathrm{ub} c 0 \mathrm{t}\right.$ ro t2 $\left.),\left(\mathrm{co},\left.\right|_{-}\right)\right)$in

let 1 t3 $:=\left(\left.\right|_{-},\left(\left(\mathrm{ub}^{\prime} 1^{\prime} \mathrm{t} r \mathrm{r}^{\mathrm{t}}\right), \mathrm{c}^{\prime}\right)\right)$ in

let $r t 1:=(((u b c 0$ t $r 1$ t5 $),(c 0$, k1 $)),(($ ub c1 t r0 t4 $),(c 1, k 0)))$ in

let $\mathrm{rt2}:=\left(\left.\right|_{-},\left(\left(\mathrm{ub} \mathrm{co}^{\prime} \mathrm{t} \mathrm{r} 1 \mathrm{t} 5\right), \mathrm{co}^{\prime}\right)\right)$ in

let $\mathrm{rt3}:=\left((\mathrm{ub} \mathrm{c} 1 \mathrm{t} \mathrm{r} 0 \mathrm{t} 4),\left(\mathrm{c} 1,\left.\right|_{-}\right)\right)$in

let $b 0:=($ acc co t ro t2 $)$ in

let $\mathrm{b} 1:=(\mathrm{acc} c 1$ t $r 0$ t4 $)$ in

let $b 0^{\prime}:=\left(\right.$ acc $c 0^{\prime}$ t $r 1$ t5 $)$ in

let $b 1^{\prime}:=\left(a c c c 1^{\prime} t r 1\right.$ t3 $)$ in

$(z++[m s g(b l c 0$ t ro), msg (bl c1't $r 1)$, msg (If b0\& b1' then It1 else (If b0 then lt2 else

(If b1' then lt3 else $\left.\left.\left(\left.\right|_{-},\left.\right|_{-}\right)\right)\right)$)]) $\sim$

$(\mathrm{z}++[\mathrm{msg}(\mathrm{bl} c 1 \mathrm{t} \mathrm{r} 0), \operatorname{msg}(\mathrm{bl} \mathrm{c0}$ t $r 1)$, msg (If b1\& b0' then rt1 else (If b0' then rt2 else

(If b1 then rt3 else $\left.\left.\left(\left.\right|_{-},\left.\right|_{-}\right)\right)\right)$)]). 
We formalize the Proposition 4.9, which states that the frames of the authentication phase are computationally indistinguishable below.

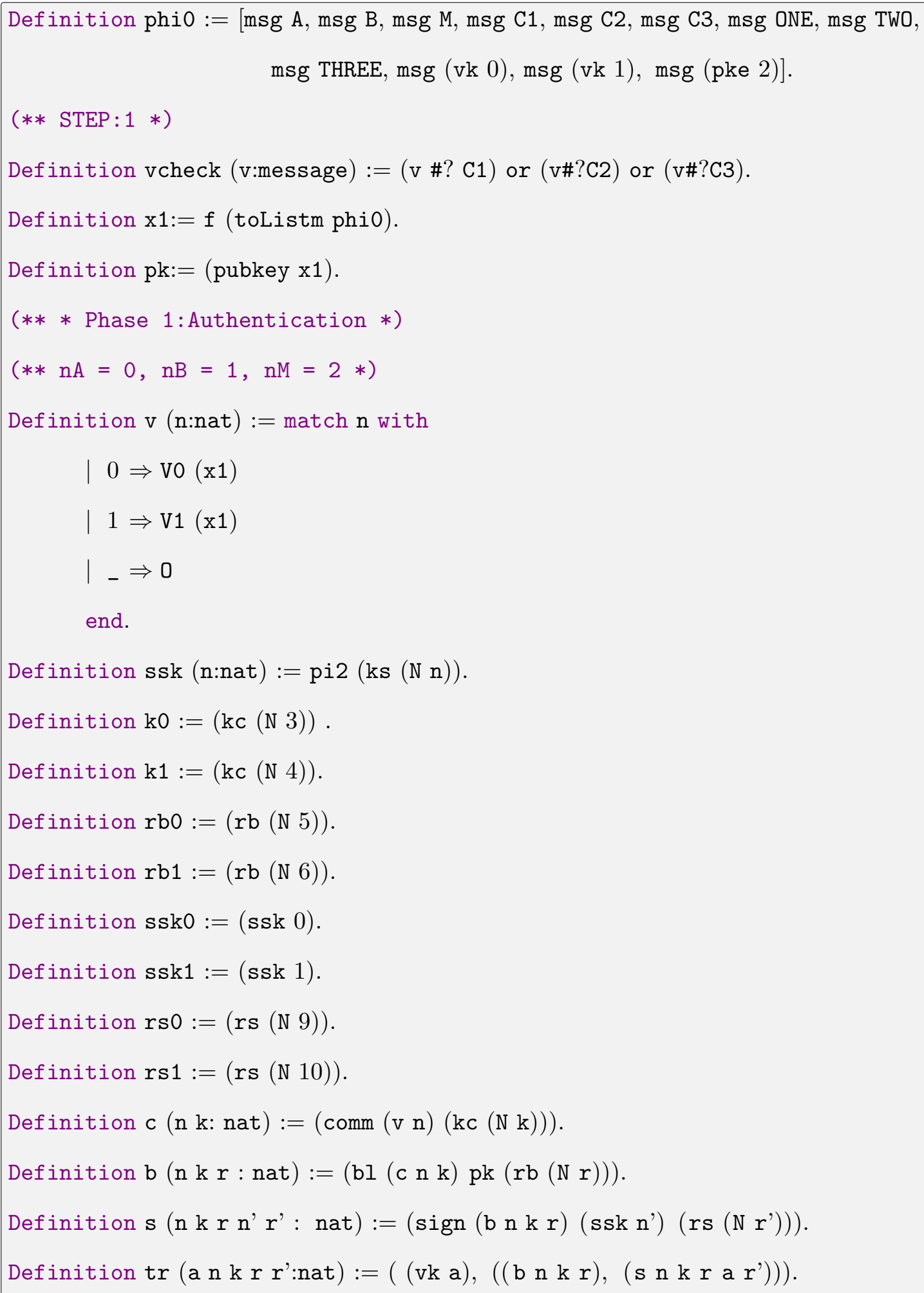




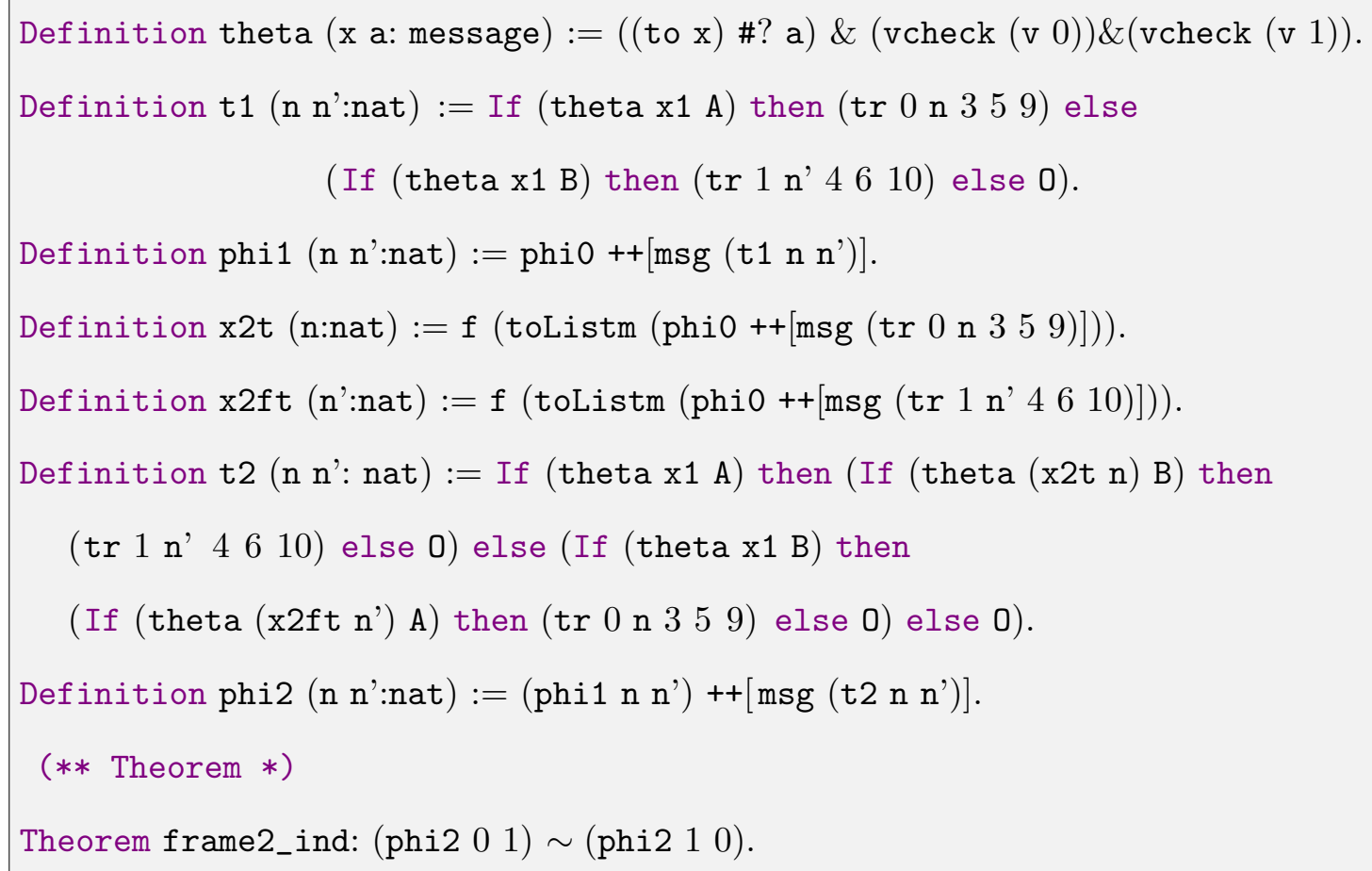

We formalize the Lemma 4.12 which captures the fact that the frames of the voting phase even if the attacker replaces one of the ballots of the honest voters with his computation below.

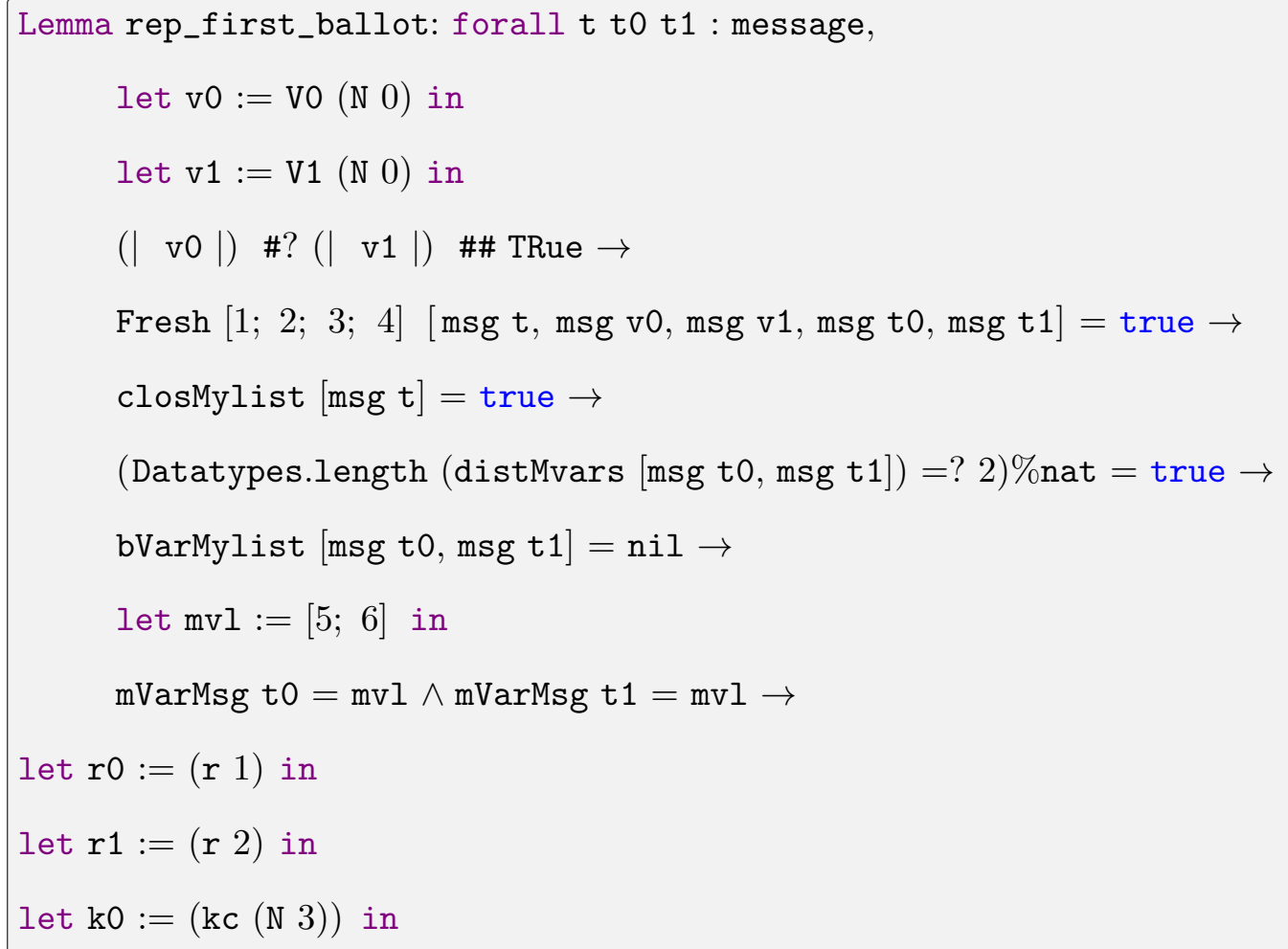




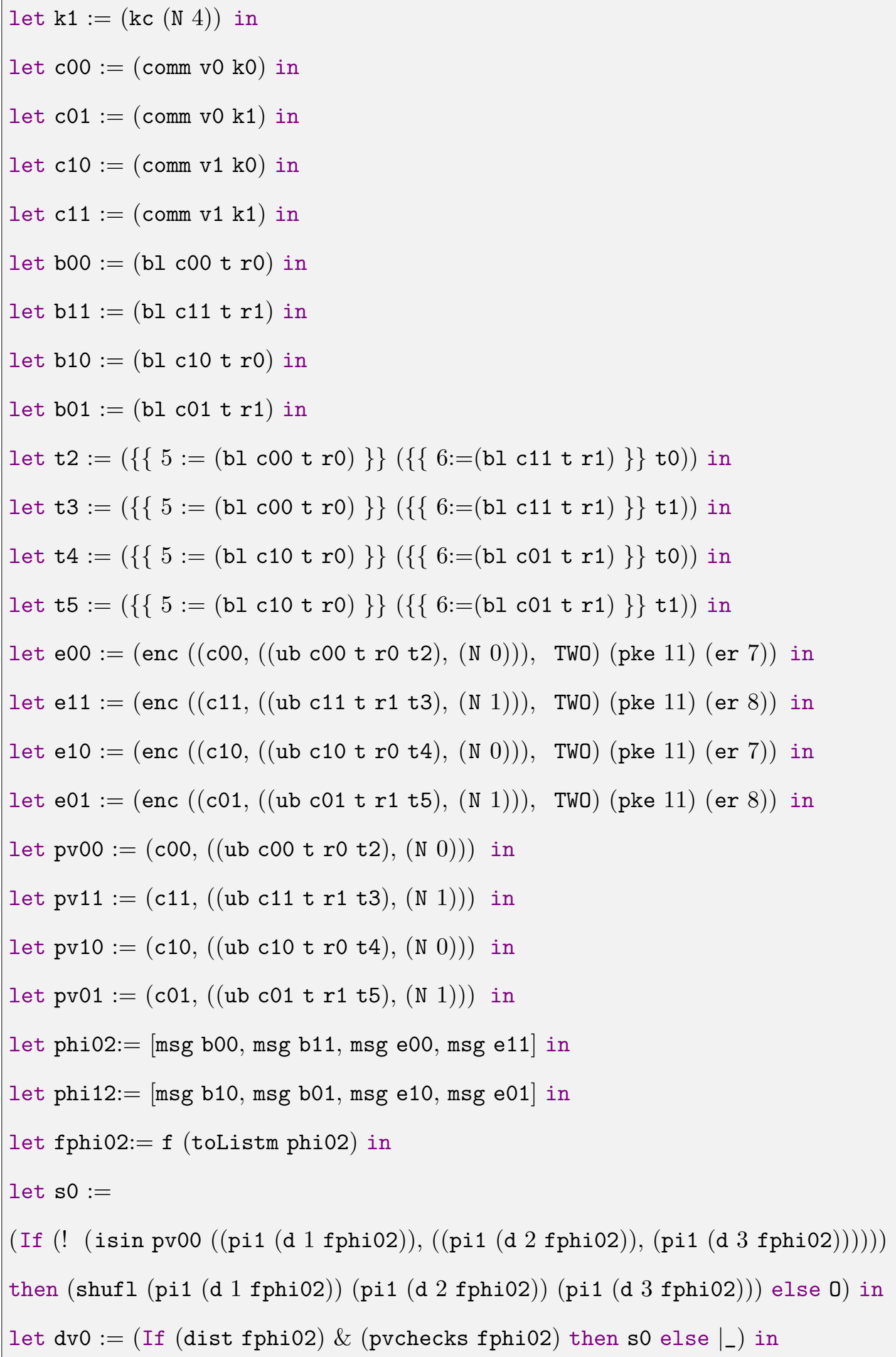




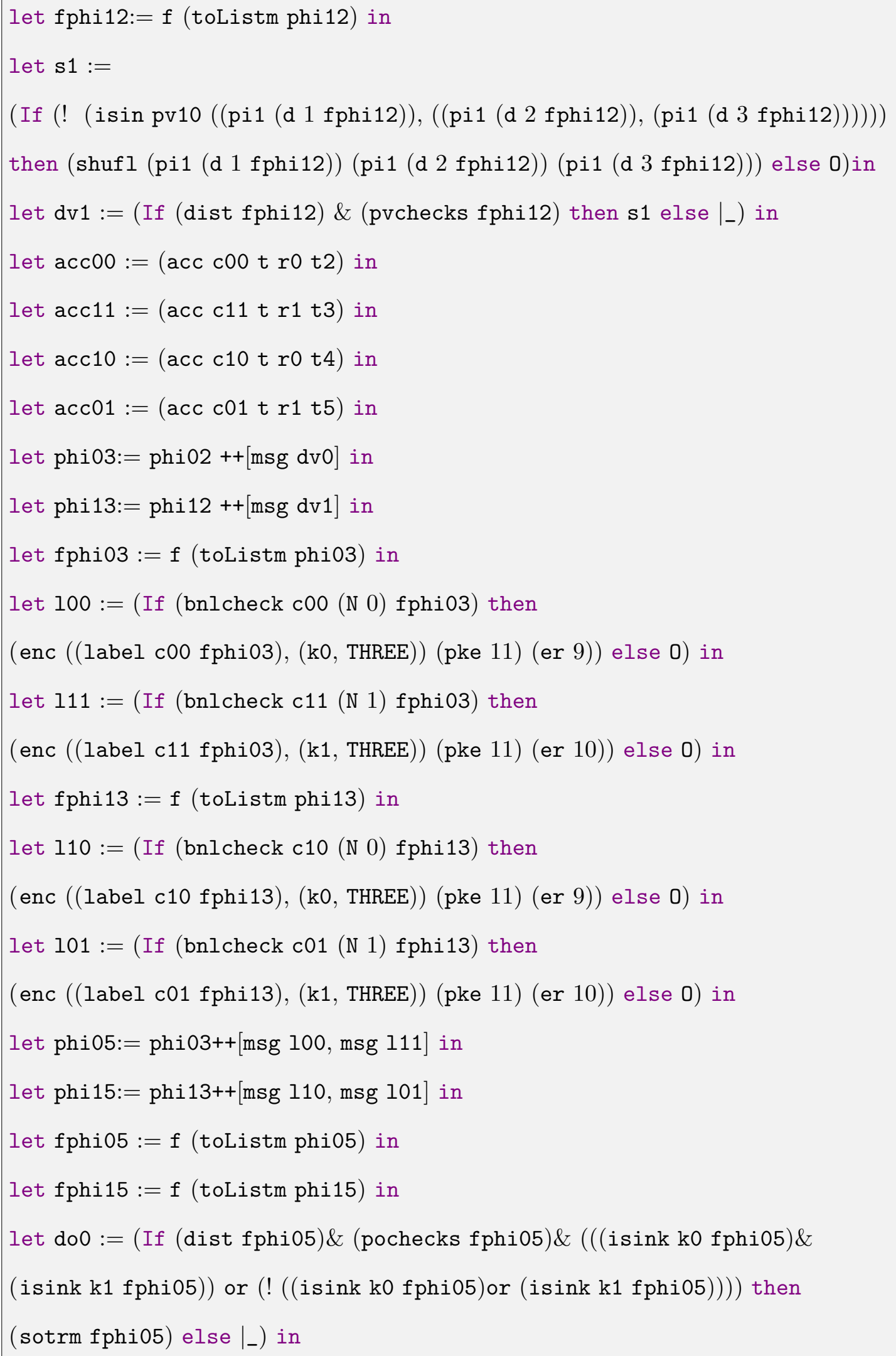




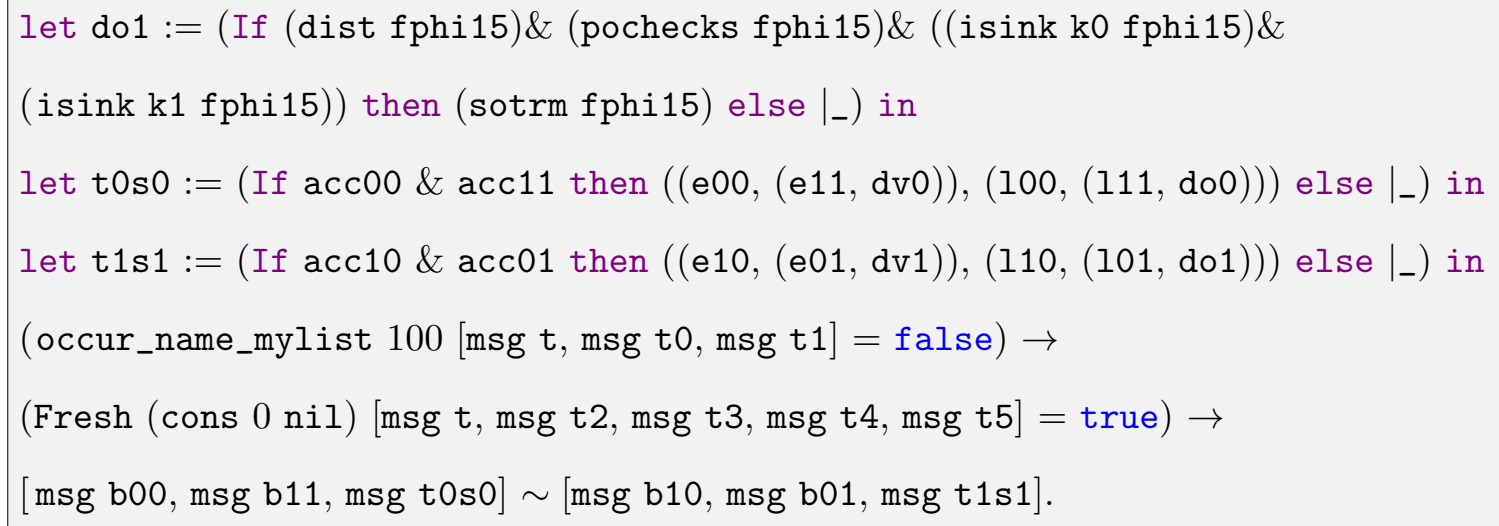

Similarly, it is not hard to see the formalization of the other lemmas of the chapter 4 .

\subsection{Authenticated Diffie-Hellman Key Exchange}

We apply our core axioms to rather different purpose: authentication. We consider an authenticated Diffie-Hellman key exchange protocol which is a simplified version of the station-to-station protocol. Note that the original station-to-station protocol contains key-confirmation as well using encryption; we omit that now to keep syntax simple. Our version of the protocol is the following:

- A group description $G$ and a group generator element $g$ are generated honestly, according to a randomized algorithm, and made public. Public verification key, secret signing key pairs are generated honestly for honest agents and the verification keys are made public.

- The Initiator, $A$, selects a responder $B$, generates a random $a$ in $\mathbb{Z}_{|g|}$ and sends $\left\langle A, B, g^{a}\right\rangle$.

- The Responder, $B$, receives $g^{a}$, generates a random $b$ in $\mathbb{Z}_{|g|}$ and sends $\left\langle g^{b}, \operatorname{sign}\left(s k_{B},\left\langle A, g^{b}, g^{a}\right\rangle, r\right)\right\rangle$, and computes $\left(g^{a}\right)^{b}$.

- The Initiator receives $\left\langle g^{b}, \operatorname{sign}\left(s k_{B},\left\langle A, g^{b}, g^{a}\right\rangle, r\right)\right\rangle$, verifies the signature, computes $\left(g^{b}\right)^{a}$, and sends $\operatorname{sign}\left(s k_{A},\left\langle B, g^{a}, g^{b}\right\rangle, r^{\prime}\right)$. 


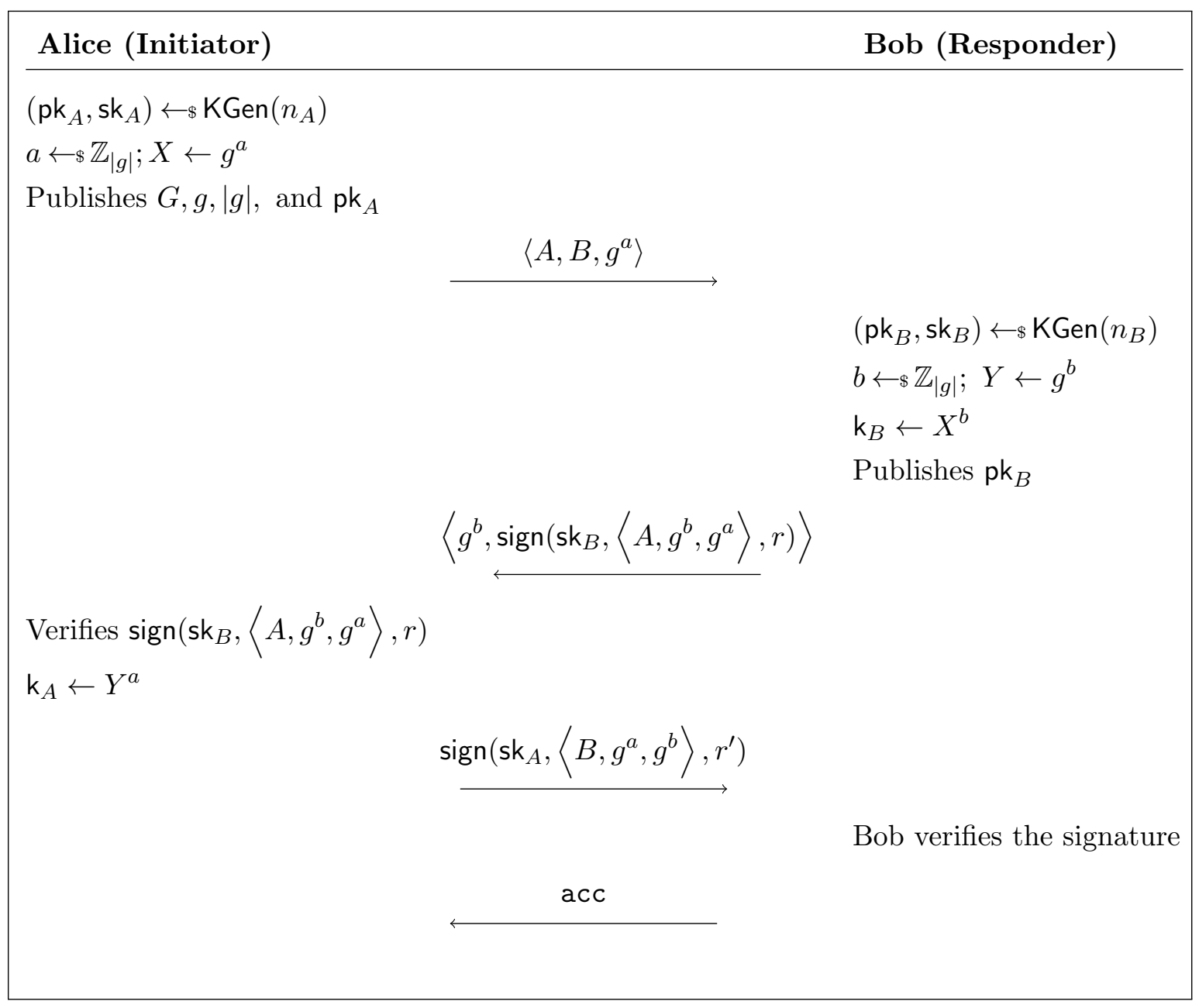

Figure 5.1: Authenticated Diffie-Hellman key exchange protocol

- The Responder receives $\operatorname{sign}\left(s k_{A},\left\langle B, g^{a}, g^{b}\right\rangle, r^{\prime}\right)$, verifies the signature, and outputs acc.

Here we can think of triples being constructed from pairs: $\langle x, y, z\rangle:=\langle x,\langle y, z\rangle\rangle$, and for projecting on the components, let $\tau_{1}:=\pi_{1}, \tau_{2}:=\pi_{1} \circ \pi_{2}, \tau_{3}:=\pi_{2} \circ \pi_{2}$.

Real-or-random secrecy for the shared keys can be verified the same way as for the DH protocol with the help of the Decisional Diffie-Hellman (DDH) assumption. 


\subsubsection{Modeling DDH assumption in the CCSA framework}

We recall the DDH assumption from Section 6 in [BCEO19]. Accordingly, we shall also include in $\mathcal{F}$ the following function symbols:

\begin{tabular}{|lrl|}
\hline$r(-):$ & message $\rightarrow$ message \\
ggen $\left(_{-}\right):$ & message $\rightarrow$ message \\
$\exp _{-}\left({ }_{-},{ }_{-}\right):$ & message $\times$message $\times$message $\rightarrow$ message \\
\hline
\end{tabular}

The function symbol ggen is for the algorithm that generates a pair consisting of the description of a cyclic group $G$ and a generator $g$ of the group. We shall write $\mathrm{G}\left({ }_{-}\right)$ for $\pi_{1}(\operatorname{ggen}(-))$, and $\mathrm{g}(-)$ for $\pi_{2}(\operatorname{ggen}(-))$. $\mathrm{r}$ is to denote the algorithm that generates an exponent randomly. We specified them as being given on message, but honest agents shall only apply them on names in $\mathcal{N}$.

The subscript of exp takes $G$ that stands for a cyclic group, the first argument $g$ is for an element of the group, and the second argument is the exponent. We shall use the abbreviations $g^{a} \stackrel{\text { def }}{=} \exp _{G}(g, a)$ and $g^{a b} \stackrel{\text { def }}{=}\left(g^{a}\right)^{b}$. Note that we do not write $G$ explicitly in the abbreviation.

$$
\begin{aligned}
& \text { DDH: fresh }\left(n, n_{1}, n_{2}, n_{3}\right) \longrightarrow \\
& \left(\mathrm{G}(n), \mathrm{g}(n), \mathrm{g}(n)^{\mathrm{r}\left(n_{1}\right)}, \mathrm{g}(n)^{\mathrm{r}\left(n_{2}\right)}, \mathrm{g}(n)^{\mathrm{r}\left(n_{1}\right) \mathrm{r}\left(n_{2}\right)}\right) \sim\left(\mathrm{G}(n), \mathrm{g}(n), \mathrm{g}(n)^{\mathrm{r}\left(n_{1}\right)}, \mathrm{g}(n)^{\mathrm{r}\left(n_{2}\right)}, \mathrm{g}(n)^{\mathrm{r}\left(n_{3}\right)}\right)
\end{aligned}
$$

Figure 5.2: Axiom that formalizes the DDH assumption

That is, this property postulates that an adversary cannot distinguish $\mathrm{g}(n)^{\mathrm{r}\left(n_{1}\right) \mathbf{r}\left(n_{2}\right)}$ from $\mathrm{g}(n)^{\mathrm{r}\left(n_{3}\right)}$ even if the items $\mathrm{G}(n), \mathrm{g}(n), \mathrm{g}(n)^{\mathrm{r}\left(n_{1}\right)}, \mathrm{g}(n)^{\mathrm{r}\left(n_{2}\right)}$ are disclosed.

The proof of validity of the DDH axiom is straight-forward and has been presented in [BCEO19]. 


\subsubsection{Modeling UF-CMA in the CCSA framework}

We recall the modeling of the UF-CMA assumption (see 3.3.3) of the signatures in the CCSA framework from [BCEO19] below:

Let $n$ be a name and let $t, u$ be closed terms such that all occurrences of $\operatorname{ssk}(n)$ in $t, u$ can be enumerated as $\operatorname{sign}\left(\operatorname{ssk}(n), t_{1}, \mathrm{r}_{\mathrm{s}}\left(n_{1}\right)\right), \operatorname{sign}\left(\operatorname{ssk}(n), t_{2}, \mathrm{r}_{\mathrm{s}}\left(n_{2}\right)\right), \ldots$,

$\operatorname{sign}\left(\operatorname{ssk}(n), t_{\ell}, r_{\mathrm{s}}\left(n_{\ell}\right)\right)$. The term ssk $(n)$ does not occur in any other form in $t, u$, and all other occurrences of $n$ in $t, u$ are of the form $\operatorname{vk}(n)$.

Let $b_{t, u}^{0}, b_{t, u}^{1}, \ldots b_{t, u}^{\ell}$ be defined recursively as:

$$
\begin{array}{ll}
b_{t, u}^{0} & \stackrel{\text { def }}{\equiv} \text { false } \\
b_{t, u}^{j} & \stackrel{\text { def }}{=} \text { if } \mathrm{EQ}\left(t, t_{j}\right) \text { then } \operatorname{ver}(\mathbf{v k}(n), t, u) \text { else } b_{t, u}^{j-1}
\end{array}
$$

UF-CMA: The axiom schema is $\operatorname{ver}(\operatorname{vk}(n), t, u)=b_{t, u}^{\ell}$.

Figure 5.3: Axiom that formalizes the UF-CMA assumption of signatures

The UF-CMA axiom captures the fact that if $t$ is one of $t_{j}$, the signature of which appears in $t$ or $u$, then the RHS outputs $\operatorname{ver}(\operatorname{vk}(n), t, u)$. If $t$ is neither of $t_{j}$, then the RHS outputs false, expressing the idea that no signature of a new $t$ can be created.

The validity proof of UF-CMA has been presented in [BCEO19].

\subsubsection{Formalizing the authentication of the STS protocol}

We shall show that with the help of the UF-CMA axiom, we can also prove authentication of the authenticated key exchange. We concentrate on the responder's noninjective authentication of the initiator, and the initiator's authentication of the responder can be handled similarly. We assume that there are two honest agents $A$ and $B$. Public key, secret key pairs $\left(\operatorname{vk}\left(n_{A}\right), \operatorname{ssk}\left(n_{A}\right)\right)$ and $\left(\operatorname{vk}\left(n_{B}\right), \operatorname{ssk}\left(n_{B}\right)\right)$ are generated honestly for $A$ and $B$. respectively. For simplicity, we assume that all sessions of $A$ are initiator sessions and all sessions of $B$ are responder sessions. We also assume 
that there are no other agents, (this is actually not really a restriction because the adversary can simulate other agents), but there can be other agent id's and associated public keys. We assume that finite number of agent id - public key pairs $\left(A, \operatorname{vk}\left(n_{A}\right)\right)$, $\left(B, \operatorname{vk}\left(n_{B}\right)\right)$ (for honest agents) and $\left(Q_{1}, \mathrm{vk}\left(c_{1}\right)\right),\left(Q_{2}, \mathrm{vk}\left(c_{2}\right)\right), \ldots$ (for dishonest agents) are publicly available to associate agent id's with public keys. The function symbols $c_{1}, c_{2}$, etc are adversarial constants. We assume that the agent ids $A, B, Q_{1}, Q_{2}, \ldots$ are pairwise distinct.

When $A$ is instructed to start an initiator session then it is given the agent id of the responder. $A$ checks that the agent name is in the list of available agent ids and can then extract the corresponding public keys. In order to simplify presentation, we assume the following abbreviations to accomplish the above:

$$
\operatorname{check}(Q)=\mathrm{EQ}(Q, A)\|\mathrm{EQ}(Q, B)\| \mathrm{EQ}\left(Q, Q_{1}\right) \| \cdots
$$

and

$$
\begin{gathered}
\operatorname{vkey}(Q)=\text { if } \operatorname{EQ}(Q, A) \text { then } \operatorname{vk}\left(n_{A}\right) \\
\text { else if } \operatorname{EQ}(Q, B) \text { then } \operatorname{vk}\left(n_{B}\right) \\
\text { else if } \operatorname{EQ}\left(Q, Q_{1}\right) \text { then } \operatorname{vk}\left(c_{1}\right) \\
\vdots \\
\text { else } \mathbf{0}
\end{gathered}
$$

The responder similarly checks if the initiator in the first message is in the list of available agent ids.

We present a transition diagram that represents the authenticated DH protocol with two participants, an initiator $A$ and a responder $B$, in Figure 5.4. For simplicity, we illustrate the case when there is only one initiator session $i d_{1}$ and one responder session $i d_{2}$. The diagram illustrates three possible branches of the protocol. The right-most branch simulates the situation where the initiator A moves first and then the responder B moves next whereas the left-most branch simulates the other sce- 
nario that $B$ moves first followed by $A$. The middle branch illustrates the scenario when all the moves of $A$ happen before the moves of $B$. The frame $\Phi_{0}$ represents the initial knowledge of the attacker which includes a description of a cyclic group $\mathrm{G}(n)$, a generator of the group $\mathrm{g}(n)$, the honest agent-ids, $A, B$, and their respective public keys $\mathrm{vk}\left(n_{A}\right)$ and $\mathrm{vk}\left(n_{B}\right)$. The initial frame also includes finite number of ids of dishonest agents, $Q_{1}, Q_{2}, \cdots$ and their public keys $\mathrm{vk}\left(c_{1}\right), \mathrm{vk}\left(c_{2}\right), \cdots$ respectively. On the input message $x_{i}$, the conditions $\theta_{i}^{1}$ checks if the input message $x_{i}$ is for $A$ and he has instructed to start a new session with agent $\mathrm{m}\left(x_{i}\right)$. This transition also checks that if the received agent id $\mathrm{m}\left(x_{i}\right)$ is valid. If the checks succeeded, then $\mathrm{A}$ extracts the public key vkey $\left(\mathrm{m}\left(x_{i}\right)\right)$ of the agent $\mathrm{m}\left(x_{i}\right) . t_{i}^{1}$ represents the $A$ 's initial message, i.e, the triple consisting of the initiator agent-id $A$, the received agent-id $\mathrm{m}\left(x_{i}\right)$, and the computed group element $g^{a}$ in the protocol. $\theta_{i j}^{1}$ checks that the received message $x_{i}$ is for $A$ and verifies that it is a valid response by checking the signature using the public key vkey $\left(\mathrm{m}\left(x_{j}\right)\right)$ which he has computed in the previous round of the protocol. $t_{i j}^{1}$ represents the final message for $A$ in the protocol, i.e, the signed message $\operatorname{sign}\left(\operatorname{ssk}\left(n_{A}\right),\left\langle\mathrm{m}\left(x_{j}\right), g^{a}, \pi_{1}\left(\mathrm{~m}\left(x_{i}\right)\right)\right\rangle, \mathrm{r}_{\mathrm{s}}\left(n_{3}\right)\right)$ of $A$ using his own secret key ssk $\left(n_{A}\right)$ and a random seed $\mathrm{r}_{\mathrm{s}}\left(n_{3}\right)$. Similarly, in $\theta_{i}^{2}$ we check that the message $x_{i}$ is for $\mathrm{B}, \tau_{1}\left(\mathrm{~m}\left(x_{i}\right)\right)$ is a valid agent and that $B$ is the intended responder. If these checks succeeded, then the responder $B$ extracts the public key $v k e y\left(\tau_{1}\left(\mathrm{~m}\left(x_{i}\right)\right)\right)$ of the agent $\tau_{1}\left(\mathrm{~m}\left(x_{i}\right)\right) . t_{i}^{2}$ is the initial response by $B$, ie, the pair that consists of the group element $g^{b}$ and the signed message $\operatorname{sign}\left(\operatorname{ssk}\left(n_{B}\right),\left\langle\tau_{1}\left(\mathrm{~m}\left(x_{i}\right)\right), b, \tau_{3}\left(\mathrm{~m}\left(x_{i}\right)\right)\right\rangle, \mathrm{r}_{\mathrm{s}}\left(n_{4}\right)\right) . \theta_{i j}^{2}$ checks that if the message $x_{i}$ is for $B$ and checks that the received message is the expected final message of the protocol by verifying the signature on the message using public key of the initiator vkey $\left(\tau_{1}\left(\mathrm{~m}\left(x_{j}\right)\right)\right)$. Of course, at the end of the protocol, the responder $B$ also sends out the message acc to indicate that the protocol has been completed. We skip other branches for lack of space.

Now, we explain how authentication can be modeled in our framework. Please note 


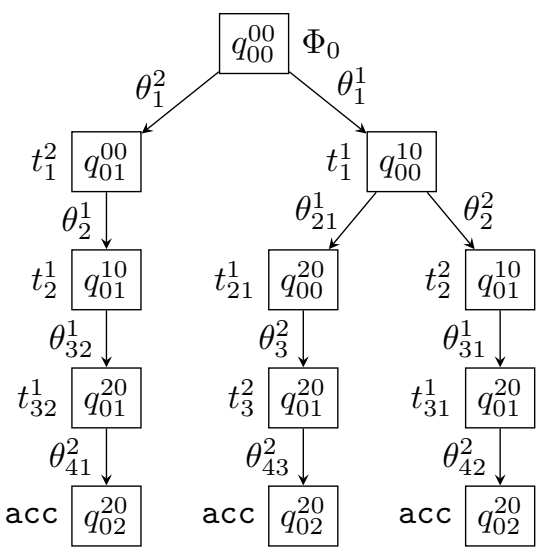

$$
\begin{aligned}
& \Phi_{0} \equiv \mathrm{G}(n), \mathrm{g}(n), A, \operatorname{vk}\left(n_{A}\right), B, \operatorname{vk}\left(n_{B}\right), Q_{1}, \operatorname{vk}\left(c_{1}\right), \cdots \\
& \theta_{i}^{1} \equiv\left(\mathrm{EQ}\left(\operatorname{to}\left(x_{i}\right), \operatorname{id} d_{1}\right) \& \mathrm{EQ}\left(\operatorname{act}\left(x_{i}\right), \text { new }\right) \& \operatorname{check}\left(\mathrm{m}\left(x_{i}\right)\right)\right. \\
& g^{a} \equiv \mathrm{g}(n)^{\mathrm{r}\left(n_{1}\right)} ; g^{b} \equiv \mathrm{g}(n)^{\mathrm{r}\left(n_{2}\right)} \\
& t_{i}^{1} \equiv\left\langle A, \mathrm{~m}\left(x_{i}\right), g^{a}\right\rangle \\
& \theta_{i j}^{1} \equiv\left(\mathrm{EQ}\left(\operatorname{to}\left(x_{i}\right), \operatorname{id}_{1}\right) \&\right. \\
& \quad \operatorname{ver}\left(v k e y\left(\mathrm{~m}\left(x_{j}\right)\right),\left\langle A, \pi_{1}\left(\mathrm{~m}\left(x_{i}\right)\right), g^{a}\right\rangle, \pi_{2}\left(\mathrm{~m}\left(x_{i}\right)\right)\right. \\
& t_{i j}^{1} \equiv \operatorname{sign}\left(\operatorname{ssk}\left(n_{A}\right),\left\langle\mathrm{m}\left(x_{j}\right), g^{a}, \pi_{1}\left(\mathrm{~m}\left(x_{i}\right)\right)\right\rangle, \mathrm{r}_{\mathrm{s}}\left(n_{3}\right)\right) \\
& \theta_{i}^{2} \equiv\left(\mathrm{EQ}\left(\operatorname{to}\left(x_{i}\right), \operatorname{id}\right) \& \operatorname{check}\left(\tau_{1}\left(\mathrm{~m}\left(x_{i}\right)\right)\right) \& \mathrm{EQ}\left(\tau_{2}\left(\mathrm{~m}\left(x_{i}\right)\right), B\right)\right. \\
& t_{i}^{2} \equiv\left\langle g^{b}, \operatorname{sign}\left(\operatorname{ssk}\left(n_{B}\right),\left\langle\tau_{1}\left(\mathrm{~m}\left(x_{i}\right)\right), g^{b}, \tau_{3}\left(\mathrm{~m}\left(x_{i}\right)\right)\right\rangle, \mathrm{r}_{\mathrm{s}}\left(n_{4}\right)\right)\right\rangle \\
& \theta_{i j}^{2} \equiv\left(\mathrm{EQ}\left(\operatorname{to}\left(x_{i}\right), \operatorname{id}_{2}\right) \&\right. \\
& \quad \operatorname{ver}\left(v k e y\left(\tau_{1}\left(m\left(x_{j}\right)\right)\right),\left\langle B, \tau_{3}\left(m\left(x_{j}\right)\right), g^{b}\right\rangle, \mathrm{m}\left(x_{i}\right)\right)
\end{aligned}
$$

Figure 5.4: Authenticated DH key exchange protocol with two agents $\mathrm{A}$ and $\mathrm{B}$ that we are considering two initiator sessions for $A$ and two responder sessions for $B$ in the following. Our methods can be easily extended to any fixed number of sessions. Responder's authentication of the initiator means that if $B$ received and verified a message that looked like $\operatorname{sign}\left(\operatorname{ssk}\left(n_{A}\right),\left\langle B, y, g^{b}\right\rangle, r^{\prime}\right)$ for some input $y$, then $A$ has a matching initiator session: $A$ has a session in which he sent $\operatorname{sign}\left(\operatorname{ssk}\left(n_{A}\right),\left\langle Q, g^{a}, x\right\rangle, r^{\prime}\right)$ for some $x$ message and agent $Q$ (and as implied by $A$ 's role also sent $\left\langle A, Q, g^{a}\right\rangle$ before that) before $B$ received it on the same branch, which is the same branch that $A$ received a message that looked like $\langle x, z\rangle$, the verification $\operatorname{ver}\left(v k e y(Q),\left\langle A, x, g^{a}\right\rangle, z\right)$ succeeded, and $\mathrm{EQ}\left(x, g^{b}\right) \& \mathrm{EQ}\left(y, g^{a}\right) \& \mathrm{EQ}(Q, B)$ is satisfied on this branch.

There are various possibilities to express responder's authentication of the initiator in our language, we present one. Namely, similarly to our modeling of secrecy, we can define an oracle query that takes a session $i d_{\alpha}$ as input, and if $i d_{\alpha}$ is a completed responder session and there is no matching initiator session in the above sense then the oracle outputs 1 (that is, true symbolically), meaning there is an attack against authentication. Otherwise it outputs 0 (that is, false symbolically). Let the protocol that ends with such an oracle query be called $\Pi_{1}^{\text {auth }}$. We can also define $\Pi_{2}^{\text {auth }}$ such that the oracle always outputs 0 . These oracles can be formalized as in the case of 
secrecy. Then, the authentication property can be formalized as:

$$
\Phi\left(\text { fold }\left(\Pi_{1}^{\text {auth }}\right)\right)=\Phi\left(\text { fold }\left(\Pi_{2}^{\text {auth }}\right)\right)
$$

Observe that we used equality, and not indistinguishability. This means that $\Pi_{1}^{\text {auth }}$ cannot output 1 with non-negligible probability.

Proposition 5.6 Let $\Pi_{1}^{\text {auth }}$ and $\Pi_{2}^{\text {auth }}$ be the two protocols as defined above. Assuming the signature scheme satisfies the UF-CMA assumption, $\Phi\left(\right.$ fold $\left.\left(\Pi_{1}^{\text {auth }}\right)\right)=\Phi\left(\right.$ fold $\left.\left(\Pi_{2}^{\text {auth }}\right)\right)$.

Proof:(Sketch.) If on a branch of $\Phi\left(\right.$ fold $\left.\left(\Pi_{1}^{\text {auth }}\right)\right)$ there is a $\boldsymbol{t r u e}$ as the final output, then by the definition of the oracle this branch lies on the true side of the branching where the condition $\mathrm{EQ}\left(\tau_{1}\left(\mathrm{~m}\left(f_{i}\left(\phi_{i}\right)\right)\right), A\right)$ and the condition $\operatorname{ver}\left(\operatorname{vkey}\left(\tau_{1}\left(\mathrm{~m}\left(f_{i}\left(\phi_{i}\right)\right)\right)\right),\left\langle B, \tau_{3}\left(\mathrm{~m}\left(f_{i}\left(\phi_{i}\right)\right)\right), g^{b}\right\rangle, \mathrm{m}\left(f_{j}\left(\phi_{j}\right)\right)\right)$ at the last move of the responder are true. Here $f_{i}\left(\phi_{i}\right)$ is the message that $B$ is supposed to have received from $A$ earlier and whose body is supposed to be $\left\langle A, B, g^{a}\right\rangle$, while the message $f_{j}\left(\phi_{j}\right)$ is supposed to be from $A$ with body $\operatorname{sign}\left(\operatorname{ssk}\left(n_{A}\right),\left\langle B, g^{a}, g^{b}\right\rangle, r^{\prime}\right)$. Since EQ $\left(\tau_{1}\left(\mathrm{~m}\left(f_{i}\left(\phi_{i}\right)\right)\right), A\right)$ is true on this branch, we can always replace $\tau_{1}\left(\mathrm{~m}\left(f_{i}\left(\phi_{i}\right)\right)\right)$ by $A$ and $v k e y\left(\tau_{1}\left(\mathrm{~m}\left(f_{i}\left(\phi_{i}\right)\right)\right)\right.$ by $\operatorname{vk}\left(n_{A}\right)$ on this branch. According to UF-CMA and EQCong, $\operatorname{ver}\left(\operatorname{vk}\left(n_{A}\right),\left\langle B, \tau_{3}\left(\mathrm{~m}\left(f_{i}\left(\phi_{i}\right)\right)\right), g^{b}\right\rangle, \mathrm{m}\left(f_{j}\left(\phi_{j}\right)\right)\right.$ can be rewritten as a branching term, which gives false (and hence the final output is also false by axioms IFMorPH and IFIDEMP and the definition of the oracle) unless $\phi_{j}$ (and hence the earlier $\phi_{i}$ ) contains a term $\operatorname{sign}\left(\operatorname{ssk}\left(n_{A}\right), t, r^{\prime}\right)$ for some $t$ such that $\mathrm{EQ}\left(\left\langle B, \tau_{3}\left(\mathrm{~m}\left(f_{i}\left(\phi_{i}\right)\right)\right), g^{b}\right\rangle, t\right)$ evaluates to true. By the role of $A, \operatorname{sign}\left(\operatorname{ssk}\left(n_{A}\right), t, r^{\prime}\right)$ must be of the form $\operatorname{sign}\left(\operatorname{ssk}\left(n_{A}\right),\left\langle\mathrm{m}\left(f_{\ell}\left(\phi_{\ell}\right)\right), g^{a}, \tau_{1}\left(\mathrm{~m}\left(f_{h}\left(\phi_{h}\right)\right)\right)\right\rangle, r^{\prime}\right)$ for an $a$ that $A$ generated at the beginning of the role in response to the message $f_{\ell}\left(\phi_{\ell}\right)$. Here $f_{h}\left(\phi_{h}\right)$ is supposed to be the initial response received by $A$. In the subsequent proof, we abbreviate $\mathrm{m}\left(f_{\ell}\left(\phi_{\ell}\right)\right)$ by $Q$. Now, we are on the true side of the $\mathrm{EQ}\left(\left\langle B, \tau_{3}\left(\mathrm{~m}\left(f_{i}\left(\phi_{i}\right)\right)\right), g^{b}\right\rangle,\left\langle Q, g^{a}, \tau_{1}\left(\mathrm{~m}\left(f_{h}\left(\phi_{h}\right)\right)\right)\right\rangle\right)$ branching. In the final oracle step, there is a branching according to $\mathrm{EQ}\left(\tau_{3}\left(\mathrm{~m}\left(f_{i}\left(\phi_{i}\right)\right)\right), g^{a}\right)$ 
\& $\mathrm{EQ}\left(\tau_{1}\left(\mathrm{~m}\left(f_{h}\left(\phi_{h}\right)\right)\right), g^{b}\right) \& \mathrm{EQ}(Q, B)$, which must fail for the oracle to output true, because otherwise there is a matching session. In the rest of the argument we show that if we are on the true side of $\mathrm{EQ}\left(\left\langle B, \tau_{3}\left(\mathrm{~m}\left(f_{i}\left(\phi_{i}\right)\right)\right), g^{b}\right\rangle,\left\langle Q, g^{a}, \tau_{1}\left(\mathrm{~m}\left(f_{h}\left(\phi_{h}\right)\right)\right)\right\rangle\right)$, then in the final oracle step the branching $\mathrm{EQ}\left(\tau_{3}\left(\mathrm{~m}\left(f_{i}\left(\phi_{i}\right)\right)\right), g^{a}\right) \& \mathrm{EQ}\left(\tau_{1}\left(\mathrm{~m}\left(f_{h}\left(\phi_{h}\right)\right)\right), g^{b}\right) \& \mathrm{EQ}(Q, B)$ can be replaced with true, and hence the final output is always false. By the equational theory of pairs, and congruence of equality, the term $\operatorname{EQ}\left(\tau_{3}\left(\mathrm{~m}\left(f_{i}\left(\phi_{i}\right)\right)\right), g^{a}\right)$ $\& \mathrm{EQ}\left(\tau_{1}\left(\mathrm{~m}\left(f_{h}\left(\phi_{h}\right)\right)\right), g^{b}\right) \& \operatorname{EQ}(Q, B)$ can be rewritten as

$$
\begin{aligned}
& \mathrm{EQ}\left(\tau_{1}\left(\left\langle Q, g^{a}, \tau_{1}\left(\mathrm{~m}\left(f_{h}\left(\phi_{h}\right)\right)\right)\right\rangle\right), \tau_{1}\left(\left\langle B, \tau_{3}\left(\mathrm{~m}\left(f_{i}\left(\phi_{i}\right)\right)\right), g^{b}\right\rangle\right)\right) \& \\
& \operatorname{EQ}\left(\tau_{2}\left(\left\langle B, \tau_{3}\left(\mathrm{~m}\left(f_{i}\left(\phi_{i}\right)\right)\right), g^{b}\right\rangle\right), \tau_{2}\left(\left\langle Q, g^{a}, \tau_{1}\left(\mathrm{~m}\left(f_{h}\left(\phi_{h}\right)\right)\right)\right\rangle\right)\right) \& \\
& \operatorname{EQ}\left(\tau_{3}\left(\left\langle Q, g^{a}, \tau_{1}\left(\mathrm{~m}\left(f_{h}\left(\phi_{h}\right)\right)\right)\right\rangle\right), \tau_{3}\left(\left\langle B, \tau_{3}\left(\mathrm{~m}\left(f_{i}\left(\phi_{i}\right)\right), g^{b}\right\rangle\right)\right)\right)
\end{aligned}
$$

As we are on the true side of $\mathrm{EQ}\left(\left\langle B, \tau_{3}\left(\mathrm{~m}\left(f_{i}\left(\phi_{i}\right)\right)\right), g^{b}\right\rangle,\left\langle Q, g^{a}, \tau_{1}\left(\mathrm{~m}\left(f_{h}\left(\phi_{h}\right)\right)\right)\right\rangle\right)$, using Example 6 and Example 7 the above checks can be replaced by

$$
\begin{aligned}
& \mathrm{EQ}\left(\tau_{1}\left(\left\langle Q, g^{a}, \tau_{1}\left(\mathrm{~m}\left(f_{h}\left(\phi_{h}\right)\right)\right)\right\rangle\right), \tau_{1}\left(\left\langle Q, g^{a}, \tau_{1}\left(\mathrm{~m}\left(f_{h}\left(\phi_{h}\right)\right)\right)\right\rangle\right)\right) \& \\
& \mathrm{EQ}\left(\tau_{2}\left(\left\langle Q, g^{a}, \tau_{1}\left(\mathrm{~m}\left(f_{h}\left(\phi_{h}\right)\right)\right)\right\rangle\right), \tau_{2}\left(\left\langle Q, g^{a}, \tau_{1}\left(\mathrm{~m}\left(f_{h}\left(\phi_{h}\right)\right)\right)\right\rangle\right)\right) \& \\
& \mathrm{EQ}\left(\tau_{3}\left(\left\langle Q, g^{a}, \tau_{1}\left(\mathrm{~m}\left(f_{h}\left(\phi_{h}\right)\right)\right)\right\rangle\right), \tau_{3}\left(\left\langle Q, g^{a}, \tau_{1}\left(\mathrm{~m}\left(f_{h}\left(\phi_{h}\right)\right)\right)\right\rangle\right)\right)
\end{aligned}
$$

which in turn can be replaced by true by EQREFL and EQCoNG. This means $\Phi\left(\right.$ fold $\left(\Pi_{1}^{\text {auth }}\right)$ ) is equal to a frame where all final outputs are false. Then, by axiom IfSAme and congruence, all branchings of the final oracle step can be collapsed and thus we obtain $\Phi\left(\right.$ fold $\left.\left(\Pi_{2}^{\text {auth }}\right)\right)$, and that is what we needed.

We formalized the authentication of the STS protocol using the axiom schema, existential unforgeability against chosen message attacks (UF-CMA) (see Section 3.3.3), and the correctness axiom of the digital signatures.

Section ds_axioms.

Axiom correctness : forall (n:nat) ( $t$ t'message), 


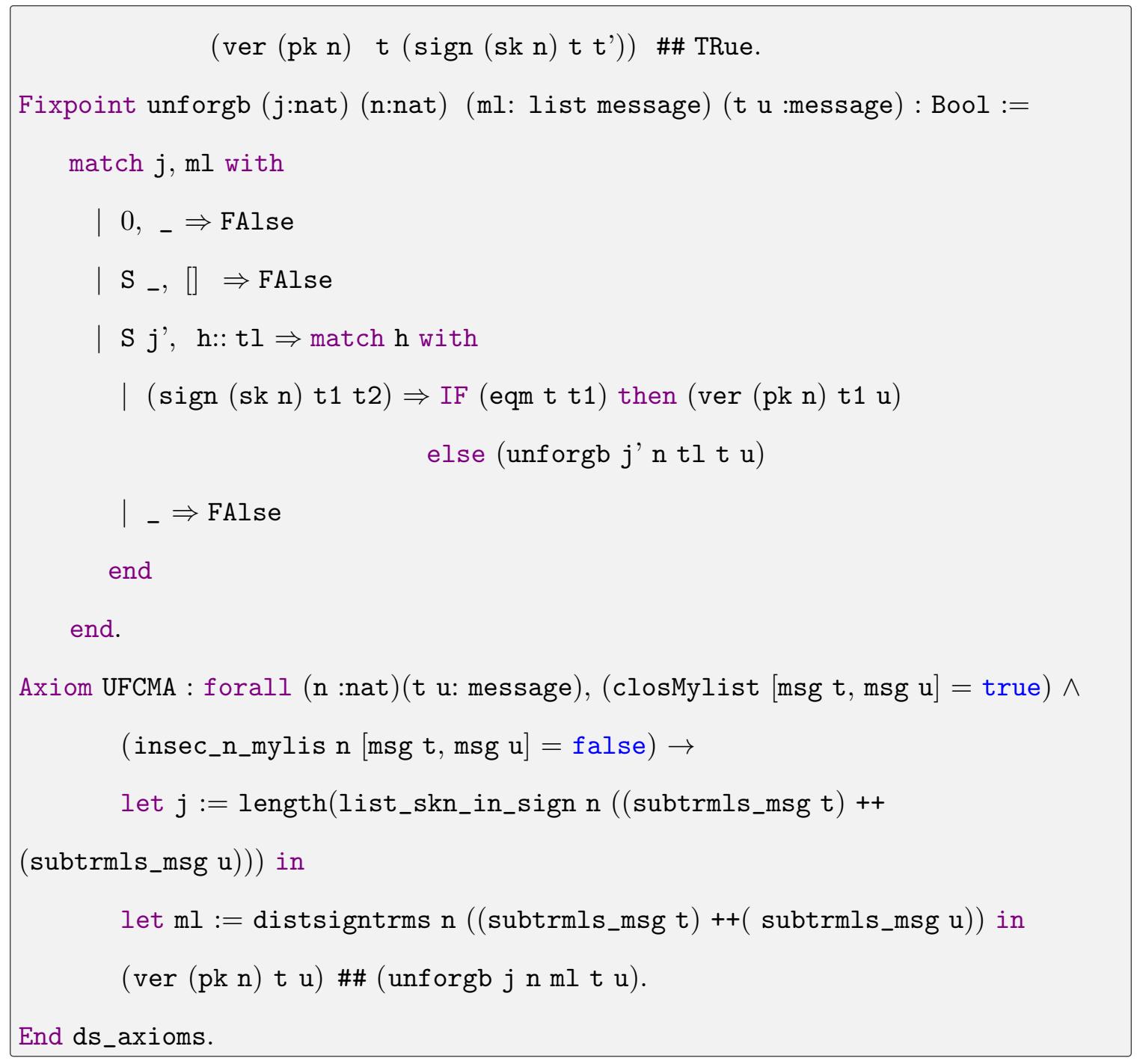

The authentication property of the STS protocol is modeled as proving the two frames $\Phi\left(\right.$ fold $\left.\left(\Pi_{1}^{\text {auth }}\right)\right)$ and $\Phi\left(\right.$ fold $\left.\left(\Pi_{2}^{\text {auth }}\right)\right)$ are equal. The frame $\Phi\left(\right.$ fold $\left.\left(\Pi_{1}^{\text {auth }}\right)\right)$ is formalized as below.

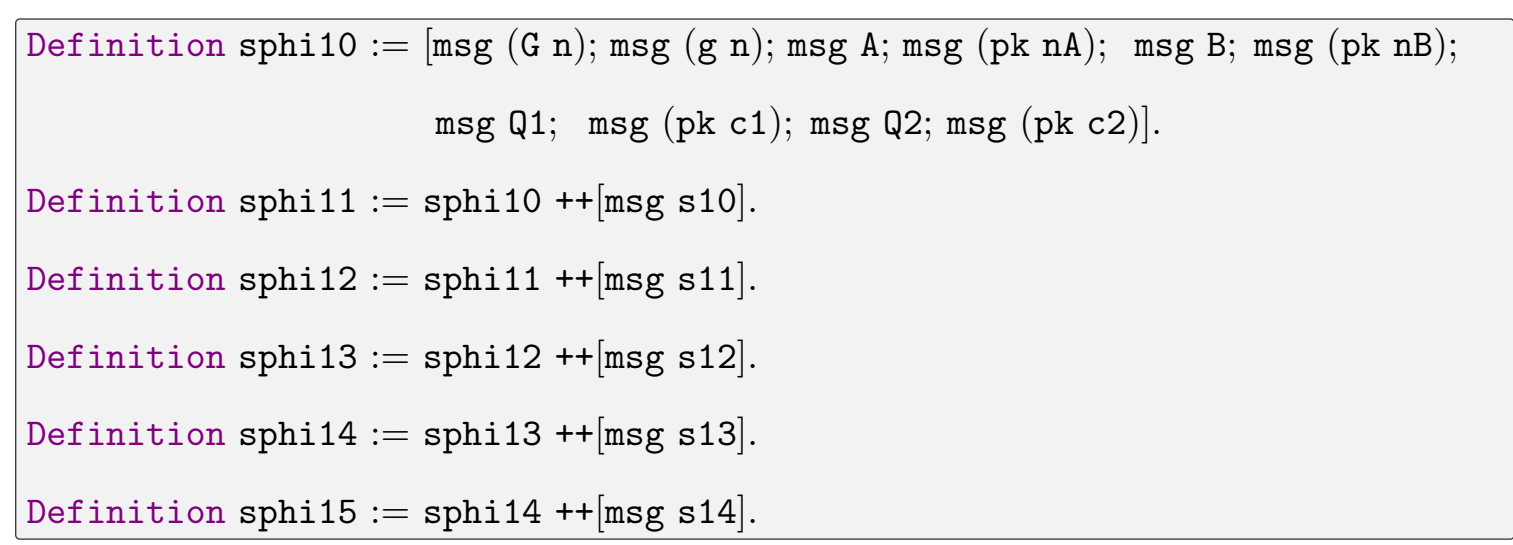


The frame sphi15 represents the frame $\Phi\left(\right.$ fold $\left.\left(\Pi_{1}^{\text {auth }}\right)\right)$ and it contains the messages that are available to the attacker at the end of the protocol. sphi10 represents the initial frame, sphi11, sphi12, sphi13, and sphi14 represent the attacker knowledge during execution of the protocol. Similarly, the frame sphi25 that represents $\Phi\left(\right.$ fold $\left.\left(\Pi_{2}^{\text {auth }}\right)\right)$ is formalized.

The authentication property of STS protocol is illustrated by the following theorem.

Theorem IND_DH_AUTH: sphi15 = sphi25.

Where the symbol $=$ represents the equality of the two frames sphi15 and sphi25.

\subsubsection{Formalizing secrecy of the STS protocol}

Secrecy. We note that secrecy of the exchanged key for STS should have a different formalization than the secrecy of the DH protocol (see Section ?? in [BCEO19]). In particular, we are interested in formalizing the property that if an honest agent completes a session ostensibly with another honest agent, then the shared key computed by the agent must be secret. This can again be modeled as an indistinguishability of two protocols $\Pi_{1}^{\text {sec }}$ and $\Pi_{2}^{\text {sec }}$. In both protocols, we enhance the transition system for the STS protocol with an oracle query for revealing a shared key. In $\Pi_{1}^{\text {sec }}$, if the oracle query is to reveal the key in a completed session $i_{\alpha}$ for $A$ ( $B$ resp.) then the oracle replies with the key computed by $A$ ( $B$ resp.) in that session. In $\Pi_{2}^{\text {sec }}$, if the oracle query is to reveal the key in a completed session id $\mathrm{d}_{\alpha}$ for $A$ ( $B$ resp.) then the oracle checks if $A$ ( $B$ resp.) completed this session ostensibly with $B$ ( $A$ resp.): if it indeed is the case then the oracle generated reveals a random key $g^{c}$, otherwise it outputs the computed key. $\Pi_{1}^{\text {sec }}$ and $\Pi_{2}^{\text {sec }}$ can be easily shown to be indistinguishable as follows.

First, $\Pi_{1}^{\text {sec }}$ can be easily transformed into a protocol, in which the oracle query before revealing $A$ 's ( $B$ 's resp.) computed secret in a completed session $\mathrm{id}_{\alpha}$ for $A(B$ 
resp.) checks if the other agent is ostensibly $B$ ( $A$ resp.). Whether the check succeeds or not, the oracle query still reveals the computed key. Now, in the branch when the aforementioned check passes, we can show in a manner similar to the proof of Proposition 5.6 that $A$ ( $B$ resp.) must be exchanging the secret with one of the two sessions of $B$ ( $A$ resp.) and the secret must be of the form $\left(g^{b}\right)^{a}\left(\left(g^{a}\right)^{b}\right.$ resp.) where $a$ and $b$ are the exponents in the corresponding sessions of $A$ and $B$. Thus, the folded term representing the protocol $\Pi_{1}^{\text {sec }}$ can be transformed into one in which the oracle query results in 5 different possible conditional outputs, 4 for the keys exchanged between the honest agents and one for all other cases. Similarly, the folded term representing the protocol $\Pi_{2}^{\text {sec }}$ can also be transformed into one in which the oracle query results in 5 different possible conditional outputs, 4 of them being $g^{c}$ when the two honest agents are involved in the exchange and one for all other cases. Now, these two terms can be proved to be indistinguishable using the computational DDH assumption.

\subsection{Summary}

In this chapter, we presented the mechanization of the proofs of vote privacy of the FOO electronic protocols presented in Chapter 4. We also presented the mechanization of the proofs of the authentication and the secrecy of STS protocol presented in [BCEO19]. For the FOO voting protocol and STS protocol, we have considered only one session each of responder and initiator to keep the formulas small. 


\section{Chapter 6}

\section{Conclusion}

We analyzed the FOO electronic voting protocol for vote privacy in the provable security model using the computationally complete symbolic attacker (CCSA) framework. As part of the analysis, we showed that security properties of commitments and blind signatures could be faithfully translated into axioms in the CCSA framework. We demonstrated that the framework is effective in that it revealed new attacks on the FOO protocol and could be used to prove the modified FOO protocol secure.

In order to achieve higher assurances, realizing the fact that the handwritten proofs are erring, we have mechanized the proofs of vote privacy of the FOO protocol in the Coq theorem prover. To demonstrate the effectiveness of our mechanization techniques, we formalize the proofs of the authentication and the secrecy of the STS protocol, which initially appeared in the publication [BCEO19].

Future work. As part of future work, we plan to investigate expressing and verifying stronger privacy properties of receipt-freeness and coercion-resistance for electronic voting protocols in the CCSA framework. We plan to, utilizing our mechanization techniques, formalize the proofs in Coq proof assistant. We also plan to investigate the automation of the verification tasks. 


\section{Bibliography}

$\left[\mathrm{ABD}^{+} 15\right]$ David Adrian, Karthikeyan Bhargavan, Zakir Durumeric, Pierrick Gaudry, Matthew Green, J. Alex Halderman, Nadia Heninger, Drew Springall, Emmanuel Thomé, Luke Valenta, Benjamin VanderSloot, Eric Wustrow, Santiago Zanella-Béguelin, and Paul Zimmermann. Imperfect forward secrecy: How diffie-hellman fails in practice. In Proceedings of the 22Nd ACM SIGSAC Conference on Computer and Communications Security, CCS '15, pages 5-17, New York, NY, USA, 2015. ACM.

[ABF17] Martín Abadi, Bruno Blanchet, and Cédric Fournet. The applied pi calculus: Mobile values, new names, and secure communication. Journal of the ACM, pages 1-41, 2017.

[Adi08] Ben Adida. Helios: Web-based open-audit voting. In Proceedings of the 17 th Conference on Security Symposium, SS'08, pages 335-348. USENIX Association, 2008.

[AF01] M. Abadi and C. Fournet. Mobile values, new names, and secure communication. In POPL'01, pages 104-115. ACM, 2001.

[ANN06] Michel Abdalla, Chanathip Namprempre, and Gregory Neven. On the (im)possibility of blind message authentication codes. In Topics in Cryptology, pages 262-279, 2006. 
[AR02] M. Abadi and P. Rogaway. Reconciling Two Views of Cryptography (The Computational Soundness of Formal Encryption). Journal of Cryptology, 15(2):103-127, 2002.

[BAS12a] G. Bana, P. Adão, and H. Sakurada. Computationally Complete Symbolic Attacker in Action. In Foundations of Software Technology and Theoretical Computer Science, pages 546-560, 2012.

[BAS12b] G. Bana, P. Adão, and H. Sakurada. Symbolic Verification of the Needham-Schroeder-Lowe Protocol, 2012. http://web.ist.utl.pt/pedro.adao/pubs/drafts/nsl-long.pdf.

$\left[\mathrm{BBD}^{+} 17\right]$ Benjamin Beurdouche, Karthikeyan Bhargavan, Antoine DelignatLavaud, Cédric Fournet, Markulf Kohlweiss, Alfredo Pironti, Pierre-Yves Strub, and Jean Karim Zinzindohoue. A messy state of the union: taming the composite state machines of TLS. Commun. ACM, 60(2):99-107, 2017.

[BC04] Yves Bertot and Pierre Castéran. A Brief Overview, pages 1-11. Springer Berlin Heidelberg, Berlin, Heidelberg, 2004.

[BC16] Gergei Bana and Rohit Chadha. Verification methods for the computationally complete symbolic attacker based on indistinguishability. Cryptology ePrint Archive, Report 2016/069, 2016.

[BCE18] Gergei Bana, Rohit Chadha, and Ajay Kumar Eeralla. Formal analysis of vote privacy using computationally complete symbolic attacker. In Computer Security - 23rd European Symposium on Research in Computer Security, ESORICS 2018, pages 350-372, 2018.

[BCEO19] Gergei Bana, Rohit Chadha, Ajay Kumar Eeralla, and Mitsuhiro Okada. Verification methods for the computationally complete symbolic attacker 
based on indistinguishability. ACM Trans. Comput. Logic, 21(1):2:12:44, October 2019.

$\left[\mathrm{BCG}^{+} 15\right]$ D. Bernhard, V. Cortier, D. Galindo, O. Pereira, and B. Warinschi. Sok: A comprehensive analysis of game-based ballot privacy definitions. In IEEE Symposium on Security and Privacy, pages 499-516, 2015.

[BCL12] G. Bana and H. Comon-Lundh. Towards Unconditional Soundness: Computationally Complete Symbolic Attacker. In First International conference on Principles of Security and Trust, pages 189-208, 2012.

[BCL14] G. Bana and H. Comon Lundh. A computationally complete symbolic attacker for equivalence properties. In ACM conference on Computer and Communications Security, pages 609-620, 2014.

$\left[\mathrm{BDF}^{+} 14\right]$ Karthikeyan Bhargavan, Antoine Delignat-Lavaud, Cédric Fournet, Alfredo Pironti, and Pierre-Yves Strub. Triple handshakes and cookie cutters: Breaking and fixing authentication over TLS. In 2014 IEEE Symposium on Security and Privacy, SP 2014, Berkeley, CA, USA, May 18-21, 2014, pages 98-113, 2014.

[BDPR98] M. Bellare, A. Desai, D. Pointcheval, and Ph. Rogaway. Relations Among Notions of Security for Public-Key Encryption Schemes. In CRYPTO’98, LNCS. Springer, 1998.

[Ben87] Josh Daniel Cohen Benaloh. Verifiable Secret-ballot Elections. PhD thesis, Yale University, 1987.

[BGHZB11] G. Barthe, B. Grégoire, S. Heraud, and S. Zanella-Béguelin. ComputerAided Security Proofs for the Working Cryptographer. In CRYPTO 2011, volume 6841 of $L N C S$, pages 71-90. Springer, 2011. 
[BHO13] G. Bana, K. Hasebe, and M. Okada. Computationally complete symbolic attacker and key exchange. In ACM conference on Computer and Communications Security, pages 1231-1246, 2013.

[Bla05] B. Blanchet. An Automatic Security Protocol Verifier based on Resolution Theorem Proving (invited tutorial). In $C A D E^{\prime} 05$, Tallinn, Estonia, July 2005.

[Bla08a] B. Blanchet. A Computationally Sound Mechanized Prover for Security Protocols. IEEE Transactions on Dependable and Secure Computing, 5(4):193-207, 2008.

[Bla08b] B. Blanchet. A computationally sound mechanized prover for security protocols. IEEE Transactions on Dependable and Secure Computing, 5(4):193-207, Oct 2008.

[Bon98] D. Boneh. The Decision Diffie-Hellman Problem. In ANTS-III'98, pages 48-63. Springer-Verlag, 1998.

[BP04] M. Backes and B. Pfitzmann. Symmetric encryption in a simulatable Dolev-Yao style cryptographic library. In Proc. IEEE Computer Security Foundations workshop, 2004.

[BPW03] M. Backes, B. Pfitzmann, and M. Waidner. A composable cryptographic library with nested operations. In $C C S^{\prime} 03$, pages 220-230. ACM, 2003.

[BT94] Josh Benaloh and Dwight Tuinstra. Receipt-free secret-ballot elections (extended abstract). In ACM Symposium on Theory of Computing, pages 544-553, 1994. 
[CCCK16] Rohit Chadha, Vincent Cheval, Ştefan Ciobâcă, and Steve Kremer. Automated verification of equivalence properties of cryptographic protocols. ACM Transactions on Computational Logic, pages 1-32, 2016.

[CCK12] Rohit Chadha, Ştefan Ciobâcă, and Steve Kremer. Automated verification of equivalence properties of cryptographic protocols. In Helmut Seidl, editor, Programming Languages and Systems, pages 108-127, Berlin, Heidelberg, 2012. Springer Berlin Heidelberg.

$\left[\mathrm{CDD}^{+} 17\right]$ Véronique Cortier, Constantin Catalin Dragan, François Dupressoir, Benedikt Schmidt, Pierre-Yves Strub, and Bogdan Warinschi. Machinechecked proofs of privacy for electronic voting protocols. In IEEE Symposium on Security and Privacy, pages 993-1008, 2017.

[CDD18] Véronique Cortier, Antoine Dallon, and Stéphanie Delaune. Efficiently deciding equivalence for standard primitives and phases. In Computer Security - 23rd European Symposium on Research in Computer Security, ESORICS 2018, Barcelona, Spain, September 3-7, 2018, Proceedings, Part I, pages 491-511, 2018.

[Cha81] David L. Chaum. Untraceable electronic mail, return addresses, and digital pseudonyms. Communications of the ACM, pages 84-90, 1981.

[Chl13] A. Chlipala. Certified Programming with Dependent Types. MIT Press, 2013.

[CK17] H. Comon and A. Koutsos. Formal computational unlinkability proofs of rfid protocols. In IEEE Computer Security Foundations Symposium, pages 100-114, 2017.

[CLC08a] H. Comon-Lundh and V. Cortier. Computational Soundness of Observational Equivalence. In CCS'08, pages 109-118. ACM, 2008. 
[CLC08b] Hubert Comon-Lundh and Véronique Cortier. Computational soundness of observational equivalence. In Proceedings of the 15th ACM Conference on Computer and Communications Security, CCS '08, pages 109-118, New York, NY, USA, 2008. ACM.

[Cre08] C. Cremers. The Scyther Tool: Verification, Falsification, and Analysis of Security Protocols. In $C A V^{\prime} 08$, volume 5123 of LNCS, pages 414-418. Springer, 2008.

[CRS83] D. Chaum, Ronald L. Rivest, and Alan T. Sherman. Blind Signatures for Untraceable Payments. In Advances in Cryptology, pages 199-203, 1983.

[DPP97] Ivan B. Damgård, Torben P. Pedersen, and Birgit Pfitzmann. On the existence of statistically hiding bit commitment schemes and fail-stop signatures. Journal of Cryptology, pages 163-194, 1997.

[DRS08] Stéphanie Delaune, Mark Ryan, and Ben Smyth. Automatic verification of privacy properties in the applied pi calculus. In IFIP International conference on Trust Management, pages 263-278, 2008.

[DY83] D. Dolev and A. Yao. On the security of public key protocols. IEEE Transactions on Information Theory, 29(2):198-208, March 1983.

[ea05] A. Armando et al. The AVISPA Tool for the automated validation of internet security protocols and applications. In $C A V^{\prime} 05$, volume 3576 of LNCS, pages 281-285, 2005.

[Eer19] Ajay Kumar Eeralla. Formalizing the Proofs of Vote Privacy, the Authentication and the Secrecy of the FOO and ADH protocol in Coq. https://bitbucket.org/ajayeeralla/vote_privacy_auth_ secrecy/src/master/, 2019. Accessed: 2019-09-11. 
[FOO93] A. Fujioka, T. Okamoto, and K. Ohta. A Practical Secret Voting Scheme for Large Scale Elections. In Advances in Cryptology - AUSCRYPT'92, pages 244-251, 1993.

[FS09] M. Fischlin and D. Schröder. Security of blind signatures under aborts. In Public-Key Cryptography, pages 297-316, 2009.

[GMR88] Shafi Goldwasser, Silvio Micali, and Ronald L. Rivest. A digital signature scheme secure against adaptive chosen-message attacks. SIAM Journal on Computing, 17(2):281-308, Apr 1988.

[Gol84] Shafrira Goldwasser. Probabilistic Encryption: Theory and Applications (Partial Information, Factoring, Pseudo Random Bit Generation). PhD thesis, University of California, Berkeley, 1984. AAI8512835.

[JLO97] Ari Juels, Michael Luby, and Rafail Ostrovsky. Security of blind digital signatures. In International Cryptology Conference, pages 150-164, 1997.

[KL07] J. Katz and Y. Lindell. Introduction to Modern Cryptography. Chapman \& Hall/CRC Press, 2007.

[Kou19] Adrien Koutsos. Decidability of a sound set of inference rules for computational indistinguishability. In 32nd IEEE Computer Security Foundations Symposium, CSF 2019, Hoboken, NJ, USA, June 25-28, 2019, pages 48-61, 2019.

[KR05] Steve Kremer and Mark Ryan. Analysis of an electronic voting protocol in the applied pi calculus. In European Symposium on Programming, pages 186-200, 2005.

[Low95] Gavin Lowe. An attack on the needham-schroeder public-key authentication protocol. Inf. Process. Lett., 56(3):131-133, November 1995. 
[Low96] Gavin Lowe. Breaking and Fixing the Needham-Schroeder Public-Key Protocol using FDR. In Tools and Algorithms for the Construction and Analysis of Systems (TACAS), volume 1055 of LNCS, pages 147-166, 1996.

[MRST01] John C. Mitchell, Ajith Ramanathan, Andre Scedrov, and Vanessa Teague. Probabilistic polynominal-time process calculus and security protocol analysis. In 16th Annual IEEE Symposium on Logic in Computer Science, Boston, Massachusetts, USA, June 16-19, 2001, Proceedings, pages 3-5, 2001.

[MW04] Daniele Micciancio and Bogdan Warinschi. Completeness theorems for the abadi-rogaway language of encrypted expressions. J. Comput. Secur., 12(1):99-129, January 2004.

[Nao91] Moni Naor. Bit commitment using pseudorandomness. Journal of Cryptology, pages 151-158, 1991.

[NS78] Roger M. Needham and Michael D. Schroeder. Using encryption for authentication in large networks of computers. Commun. ACM, 21(12):993-999, December 1978.

$\left[\mathrm{PdAC}^{+} 17\right]$ Benjamin C. Pierce, Arthur Azevedo de Amorim, Chris Casinghino, Marco Gaboardi, Michael Greenberg, Cătălin Hriţcu, Vilhelm Sjöberg, and Brent Yorgey. Software Foundations. Electronic textbook, 2017. Version 5.0. http://www.cis. upenn. edu/ bcpierce/sf.

[RRI16] P. Y. A. Ryan, P. B. Rønne, and V. Iovino. Selene: Voting with transparent verifiability and coercion-mitigation. In Financial Cryptography and Data Security, pages 176-192. Springer, 2016. 
[SB13] Ben Smyth and David Bernhard. Ballot secrecy and ballot independence coincide. In European Symposium on Research in Computer Security, pages 463-480, 2013.

[Sce15] Guillaume Scerri. Proofs of security protocols revisited. Thèse de doctorat, Laboratoire Spécification et Vérification, ENS Cachan, France, January 2015.

[Sho04] Victor Shoup. Sequences of games: a tool for taming complexity in security proofs. Cryptology ePrint Archive, Report 2004/332, 2004. https://eprint.iacr.org/2004/332.

[Sma16] Nigel P. Smart. Cryptography Made Simple. Information Security and Cryptography. Springer, 2016.

[SMCB12] B. Schmidt, S. Meier, C.J.F. Cremers, and D. A. Basin. Automated analysis of diffie-hellman protocols and advanced security properties. In 25th IEEE Computer Security Foundations Symposium, CSF'12, pages 78-94, 2012.

[SS16] Guillaume Scerri and Ryan Stanley-Oakes. Analysis of key wrapping APIs: Generic policies, computational security. In IEEE Computer Security Foundations Symposium, pages 281-295, 2016.

[Yao82] A. C. Yao. Theory and application of trapdoor functions. In 23rd Annual Symposium on Foundations of Computer Science (sfcs 1982), pages 8091, Nov 1982. 


\section{VITA}

Ajay Kumar Eeralla was born in Karimnagar, Telangana, India on June $2^{\text {nd }}, 1984$, to late Shri Kondaiah Eeralla and Komuramma Eeralla. He received the BS degree majoring in Mathematics and Computer Science from Kakatiya University, the MS degree in Mathematics from Osmania University, and the MTech in Systems Analysis and Computer Applications from the National Institute of Technology (NIT), Karnataka, India. He also received an MS degree in Computer Science from Clarkson University, Potsdam, NY, USA. 